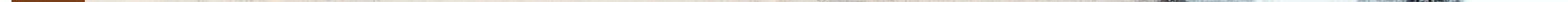




\section{COLORIMETRIC AND SPECTRAL ANALYSIS OF ROCK ART BY MEANS OF THE CHARACTERIZATION OF DIGITAL SENSORS}

Author:

Adolfo Molada Tebar
SUPERVISORS:

José Luis Lerma García

Ángel Marqués Mateu

Programme in Geomatics Engineering

Universitat PolitècnicA DE VALÈnCIA

Department of Cartographic Engineering, Geodesy and Photogrammetry

VALENCIA, OCTOBER 2020 

"The purest and most thoughtful minds are those which love color the most." - John Ruskin, The Stones of Venice

"Mere color, unspoiled by meaning, and unallied with definite form, can speak to the soul in a thousand different ways."

- Oscar Wilde 
"Colors are the feelings with which we paint our souls."

Adolfo Molada 


\section{Acknowledgments}

We could live in black and white, but fortunately, life is in color. Color is a source of emotion, of memories, of sensations. Color makes us happy. It's the same with people. Like colors, there are people who calm you down, evoke memories, embrace you, smile at you. My gratitude goes first of all to these people, who, like colors, have brought fullness to my life. Especially, to my parents, my sister and my friends, who have always been there, and will always be.

During my PhD, I had to go through moments of light and darkness. It has been a long, hard road, but it was worth it. I have learned a lot. Thank you, José Luis and Ángel, for guiding me through these years. Your contributions have been essential in bringing color to this thesis. Thanks also to a simple email. An email that changed my life. Thank you Stephen for responding positively to my request, and allowing me to take six amazing months in Leeds (UK), collaborating in the Colour Research Group at the School of Design. A dream come true! Thanks also to Zeynep, for the month stay at Mimar Sinan Fine Arts University (Istanbul, Turkey). I enjoyed the amazing sights and monuments. When I think of Istanbul, I remember colors. Thanks to all of you!

Finally, thank you very much to my colleagues in GIFLE. We have laughed, we have cried (haven't we, Inés?), but most of all we have enjoyed ourselves. Thanks Silvia and Berta. "Our meetings in Switzerland" were always the best. Thanks to my University, the Universitat Politècnica de València for the FPI scholarship that allowed me to fulfill my dream. And of course, thanks to my Department, the DICGF, which for the last years has been my second home. 



\section{Agradecimientos}

Podríamos vivir en blanco y negro, pero afortunadamente la vida es en color. El color es fuente de emoción, de recuerdos, de sensaciones. Nos hace felices. Lo mismo sucede con las personas. Como los colores, hay personas que te calman, te evocan recuerdos, te abrazan, te sonríen. Mi agradecimiento va en primer lugar a estas personas, que como los colores, han dado plenitud a mi vida. Especialmente a mis padres, mi hermana y mis amigos, que siempre han estado ahí, y siguen haciéndolo.

Durante el doctorado, han habido momentos de luz y oscuridad. Ha sido un camino largo y arduo, pero ha merecido la pena. He aprendido mucho. Gracias José Luis y Ángel por haberme guiado durante estos años. Vuestras contribuciones han sido esenciales para llenar de color esta tesis. Gracias también a un simple mail. Un mail que cambiaría mi vida. Gracias Stephen por responder afirmativamente a mi petición, y permitirme seis meses asombrosos en Leeds, UK, colaborando en el Colour Research Group de la School of Design. ¡Un sueño hecho realidad! Gracias también a Zeynep, por la estancia de un mes en Mimar Sinan Fine Arts University, Estambul, Turquía. He disfrutado de vistas y monumentos asombrosos. Cuando pienso en Estambul, pienso en colores. ¡Gracias a todos!

Por último, muchas gracias a mis compañeros en GIFLE. Hemos reído, hemos llorado (¿verdad Inés?), pero sobre todo hemos disfrutado. Gracias Silvia y Berta. 'Nuestras reuniones en Suiza' siempre fueron lo mejor. Gracias a mi universidad, la Universitat Politècnica de València por la beca FPI que me ha permitido cumplir mi sueño. Y por supuesto, gracias a mi Departamento, el DICGF, que durante los últimos años ha sido mi segunda casa. 



\section{Summary}

Cultural heritage documentation and preservation is an arduous and delicate task but not trivial, in which color plays a fundamental role. Factors such as the difficulty of reading the pigment, the complexity of the support and the variations in environmental lighting make color specification a difficult undertaking. However, the correct determination of color provides vital information on a descriptive, technical and quantitative level. Color allows a better understanding of the study area and offers vital information not only on its current state but also on the aging of the pigments.

Classical color documentation methods in archaeology were usually restricted to strictly subjective procedures, based on direct visual observations supported by color charts. However, this methodology has practical and technical limitations, affecting the results obtained in the determination of color. Nowadays, it is frequent to support classical methods with geomatics techniques, such as photogrammetry or laser scanning, together with digital image processing and enhancement techniques. The use of these novel techniques has represented a notable advance. In fact, issues such as the precise geometric description of historical objects have been solved. However, there are still aspects to be addressed, such as the correct specification of color. Although digital images allow color to be captured quickly, easily, and in a non-invasive way, the RGB data provided by the camera does not itself have a rigorous colorimetric sense. Therefore, a rigorous transformation process to obtain reliable color data from digital images is required.

This thesis proposes a novel technical solution, in which the integration of spectrophotometric and colorimetric analysis is intended as a complement to photogrammetric techniques that allow an improvement in color identification and representation of pigments with maximum reliability in 3D surveys, models and reconstructions. The proposed methodology is based on the colorimetric characterization of digital sensors, which is of novel application in cave paintings. The characterization aims to obtain the transformation equations between the device-dependent color data recorded by the camera and the independent, physically-based color spaces, such as those established by the Commission Internationale de l'Éclairage (CIE). 
The rigorous processing of color and spectral data requires software packages with specific colorimetric functionalities. Although there are different commercial software options, they do not integrate the digital image processing and colorimetric computations together. And more importantly, they do not allow the camera characterization to be carried out. Therefore, as a key aspect in this thesis is our in-house pyColourimetry software that was developed and tested taking into account the recommendations published by the CIE. pyColourimetry is an open-source code, independent without commercial ties; it allows the treatment of colorimetric and spectral data and the digital image processing, and gives full control of the characterization process and the management of the obtained data to the user.

On the other hand, this study presents a further analysis of the main factors affecting the characterization, such as the camera built-in sensor, the camera parameters, the illuminant, the regression model, and the data set used for model training. For computing the transformation equations, the literature recommends the use of polynomial equations as a regression model. Thus, polynomial models are considered as a starting point in this thesis. Additionally, a regression model based on Gaussian processes has been applied, and the results obtained by means of polynomials have been compared. Also, a new working scheme was reported which allows the automatic selection of color samples, adapted to the chromatic range of the scene. This scheme is called P-ASK, based on the $K$-means classification algorithm.

The results achieved in this thesis show that the proposed framework for camera characterization is highly applicable in documentation and conservation tasks in general cultural heritage applications, and particularly in rock art painting. It is a low-cost and non-invasive methodology that allows for the colorimetric recording from complete image scenes. Once characterized, a conventional digital camera can be used for rigorous color determination, simulating a colorimeter. Thus, it is possible to work in a physical color space, independent of the device used, and comparable with data obtained from other cameras that are also characterized. We are fully confident that the methodology proposed in this thesis will contribute significantly to improving and simplifying the correct specification of color in cultural heritage documentation and preservation tasks. 


\section{Resumen}

Las labores de documentación gráfica de arte rupestre, donde el color desempeña un aspecto fundamental, son arduas y delicadas por la dificultad de lectura del pigmento, la complejidad del soporte y las variaciones en la iluminación ambiental. La correcta determinación del color proporciona información vital a nivel descriptivo, técnico y cuantitativo, permitiendo una mayor comprensión del área de estudio y ofreciendo información no solo respecto a su estado actual sino sobre el envejecimiento de los pigmentos.

Tradicionalmente los métodos de documentación en arqueología quedaban restringidos a procedimientos estrictamente subjetivos, basados en observaciones directas a simple vista del investigador apoyadas con cartas de color. Sin embargo, esta metodología conlleva limitaciones prácticas y técnicas, afectando a los resultados obtenidos en la determinación del color. En este sentido, el empleo combinado de técnicas geomáticas, como la fotogrametría o el láser escáner, junto con técnicas de procesamiento y realce de imágenes digitales, ha supuesto un notable avance. Y aunque el empleo de estas nuevas tecnologías ha permitido resolver cuestiones como la precisa descripción geométrica de los objetos históricos, todavía quedan aspectos que resolver, como la correcta especificación del color. Aunque las imágenes digitales permiten capturar el color de forma rápida, sencilla, y no invasiva, los datos RGB proporcionados por la cámara no tienen en sí mismos un sentido colorimétrico riguroso. Se requiere la aplicación de un proceso riguroso de tranformación que permita obtener datos fidedignos del color a través de imágenes digitales.

En esta tesis se propone una solución científica novedosa y de vanguardia, en la que se persigue integrar el análisis espectrofotométrico y colorimétrico como complemento a técnicas fotogramétricas que permitan una mejora en la identificación del color y representación de pigmentos con máxima fiabilidad en levantamientos, modelos y reconstrucciones tridimensionales (3D). La metodología propuesta se basa en la caracterización colorimétrica de sensores digitales, que es de novel aplicación en pinturas rupestres. La caracterización pretende obtener las ecuaciones de transformación entre los datos de color registrados por la cámara, dependientes del dispositivo, y espacios de color independientes, de base física, como los establecidos por la Commission Internationale de l'Éclairage (CIE). 
Para el tratamiento de datos colorimétricos y espectrales de forma rigurosa se requiere disponer de un software de características técnicas muy específicas. Aunque existe software comercial, lo cierto es que realizan por separado el tratamiento digital de imágenes y las operaciones colorimétricas. No existe software que integre ambas, ni que además permita llevar a cabo la caracterización. Por tanto, como aspecto adicional y fundamental, presentamos en esta tesis el software propio que se ha desarrollado, denominado pyColourimetry, siguiendo las recomendaciones publicadas por la CIE. pyColourimetry es de código abierto, y ha sido adaptado al flujo metodológico propuesto, de modo que facilite la independencia y el progreso científico sin ataduras comerciales, permitiendo el tratamiento de datos colorimétricos y espectrales, y confiriendo al usuario pleno control del proceso y la gestión de los datos obtenidos.

Además, en este estudio se expone con detalle el análisis de los principales factores que afectan a la caracterización tales como el sensor empleado, los parámetros de la cámara durante la toma, la iluminación, el modelo de regresión, y el conjunto de datos empleados como entrenamiento del modelo. Para la determinación de las ecuaciones de transformación, la bibliografía recomienda el empleo de ecuaciones polinómicas como modelo de regresión, que es el que se ha tomado como punto de partida. Adicionalmente se ha aplicado un modelo de regresión basado en procesos Gaussianos, y se ha comparado con los resultados obtenidos mediante polinomios. También presentamos un nuevo esquema de trabajo que permite la selección automática de muestras de color, adaptado al rango cromático de la escena, que se ha denominado P-ASK, basado en el algoritmo de clasificación $K$-means.

Los resultados obtenidos en esta tesis demuestran que el proceso metodológico de caracterización propuesto es altamente aplicable en tareas de documentación y preservación del patrimonio cultural en general, y en arte rupestre en particular. Se trata de una metodología de bajo coste, no invasiva, que permite obtener el registro colorimétrico de escenas completas. Una vez caracterizada, una cámara digital convencional puede emplearse para la determinación del color de forma rigurosa, simulando un colorímetro, lo que permitirá trabajar en un espacio de color de base física, independiente del dispositivo y comparable con datos obtenidos mediante otras cámaras que también estén caracterizadas. Confiamos plenamente en que la metodología expuesta en esta tesis contribuirá a mejorar y simplificar la correcta especificación del color en tareas relacionadas con la documentación y preservación de nuestros bienes culturales. 


\section{Resum}

Les tasques de documentació gràfica d'art rupestre, on el color compleix un paper fonamental, són àrdues i delicades per la dificultat de lectura del pigment, la complexitat del suport i les variacions en la il-luminació ambiental. La correcta determinació de la color proporciona informació vital a nivell descriptiu, tècnic i quantitatiu, permetent una major comprensió de l'àrea d'estudi i oferint informació no només pel que fa al seu estat actual sinó sobre l'envelliment dels pigments.

Tradicionalment els mètodes de documentació en arqueologia quedaven restringits a procediments estrictament subjectius, basats en observacions directes a primera vista de l'investigador recolzades amb cartes de color. No obstant aixó, aquesta metodologia comporta limitacions pràctiques i tècniques, afectant els resultats obtinguts en la determinació de la color. En aquest sentit, l'ús combinat de tècniques geomàtiques, com la fotogrametria o el làser escàner, juntament amb tècniques de processament i realç d'imatges digitals, ha suposat un notable avanç. I encara que l'ús d'aquestes noves tecnologies ha permès resoldre qüestions com la descripció geomètrica precisa dels objectes histórics, encara queden aspectes per resoldre, com la correcta especificació de la color. Tot i que les imatges digitals permeten capturar el color de forma ràpida, senzilla, i no invasiva, les dades RGB proporcionades per la càmera no tenen en si mateixos un sentit colorimètric rigorós. Es requereix l'aplicació d'un procés rigorós de transformació que permeti obtenir dades fidedignes de la color a través d'imatges digitals.

En aquesta tesi es proposa una solució científica innovadora i d'avantguarda, en la qual es persegueix integrar l'anàlisi espectrofotomètric i colorimètric com a complement a tècniques fotogramètriques que permetin una millora en la identificació de la color i representació de pigments amb màxima fiabilitat en aixecaments, models i reconstruccions tridimensionals 3D. La metodologia proposada es basa en la caracterització colorimètrica de sensors digitals, que és de novell aplicació en pintures rupestres. La caracterització pretén obtenir les equacions de transformació entre les dades de color registrats per la càmera, dependents d'el dispositiu, i espais de color independents, de base física, com els establerts per la Commission Internationale de l'Éclairage (CIE). 
Per al tractament de dades colorimètriques i espectrals de forma rigorosa es requereix disposar d'un programari de característiques tècniques molt específiques. Encara que hi ha programari comercial, la veritat és que fan per separat el tractament digital d'imatges i les operacions colorimètriques. No hi ha programari que integri totes dues, ni que permeti dur a terme la caracterització. Per tant, com a aspecte addicional i fonamental en aquesta tesis, vam presentar el programari propi que s'ha desenvolupat, denominat pyColourimetry, segons les recomanacions publicades per la CIE. pyColourimetry és de codi obert, i s'adaptat al flux metodològic proposat, de manera que faciliti la independència i el progrés científic sense lligams comercials, permetent el tractament de dades colorimètriques i espectrals, i conferint a l'usuari ple control del procés i la gestió de les dades obtingudes.

A més, en aquest estudi s'exposa amb detall l'anàlisi dels principals factors que afecten la caracterització tals com el sensor emprat, els paràmetres de la càmera durant la presa, il-luminació, el model de regressió, i el conjunt de dades emprades com a entrenament d'el model. Per a la determinació de les equacions de transformació, la bibliografia recomana l'ús d'equacions polinómiques com a model de regressió, que és el que s'ha pres com a punt de partida. Addicionalment s'ha aplicat un model de regressió basat en processos Gaussians, i s'han comparat els resultats obtinguts mitjançant polinomis. També vam presentar un nou esquema de treball que permet la selecció automàtica de mostres de color, adaptat a la franja cromàtica de l'escena, que s'ha anomenat P-ASK, basat en l'algoritme de classificació $K$-means.

Els resultats obtinguts en aquesta tesi demostren que el procés metodològic de caracterització proposat és altament aplicable en tasques de documentació i preservació de el patrimoni cultural en general, i en art rupestre en particular. Es tracta d'una metodologia de baix cost, no invasiva, que permet obtenir el registre colorimètric d'escenes completes. Un cop caracteritzada, una càmera digital convencional pot emprar-se per a la determinació de la color de forma rigorosa, simulant un colorímetre, el que permetrà treballar en un espai de color de base física, independent d'el dispositiu i comparable amb dades obtingudes mitjançant altres càmeres que també estiguin caracteritzades. Confiem plenament en que la metodologia exposada en aquesta tesi contribuirà a millorar i simplificar la correcta especificació de la color en tasques relacionades amb la documentació i preservació dels nostres béns culturals. 


\section{Abbreviations}

$\begin{array}{ll}\text { BS } & \text { Based Stochastic optimization CAT } \\ \text { BSPC } & \text { Based Stochastic optimization with a Positive Constraint CAT } \\ \text { CAT02 } & \text { Chromatic Adaptation Transform 2002 (CIE) } \\ \text { CATs } & \text { Chromatic Adaptation Transforms } \\ \text { CCD } & \text { Charge Coupled Device } \\ \text { CFA } & \text { Color Filter Array } \\ \text { CIE } & \text { Commission Internationale de l'Éclairage } \\ \text { CMCCAT2000 } & \text { Color Appearance Modeling Chromatic Adaptation Transform 2000 (CIE) } \\ \text { CMFs } & \text { Color matching functions } \\ \text { CMOS } & \text { Complementary Metal Oxide Semiconductor } \\ \text { FCC } & \text { Full ColorChecker } \\ \text { GP } & \text { Gaussian process } \\ \text { ICC } & \text { International Color Consortium } \\ \text { ICV } & \text { Institut Cartogràfic Valencià } \\ \text { IEC } & \text { International Electrotechnical Commission } \\ \text { JND } & \text { Just noticeable difference } \\ \text { JS } & \text { Joined samples } \\ \text { KDP } & \text { K-means dominant patches } \\ \text { LOOCV } & \text { Leave-one-out cross-validation } \\ \text { MCMC } & \text { Markov chain Monte Carlo } \\ \text { NN } & \text { Nearest n-patches } \\ \text { P-ASK } & \text { Patch Adaptive Selection with K-means } \\ \text { PCA } & \text { Principal Component Analysis } \\ \text { Ps } & \text { Adobe Photoshop } \\ \text { RC } & \text { Remove ColorChart } \\ \text { Res } & \text { Fitted CIE XYZ residuals } \\ \text { RL } & \text { CIE XYZ LOOCV residuals } \\ \text { RMSE } & \text { Root Mean Square Error } \\ \text { SLR } & \text { Single Lens Reflex } \\ \text { SPD } & \text { Spectral Power Distribution } \\ \text { SS } & \text { Separated Samples } \\ \text { SVD } & \text { Single Value Decomposition } \\ & \end{array}$




\section{Contents}

1 Introduction $\quad 1$

1.1 Introduction . . . . . . . . . . . . . . . . . . . 2

1.2 Background and research justification $\ldots \ldots \ldots \ldots \ldots \ldots$

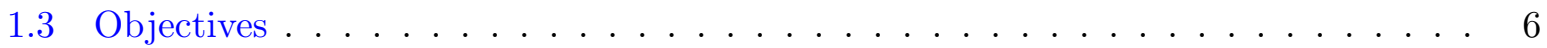

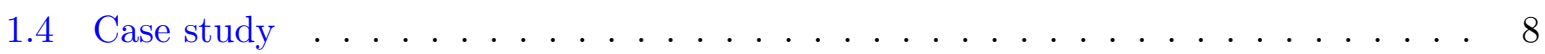

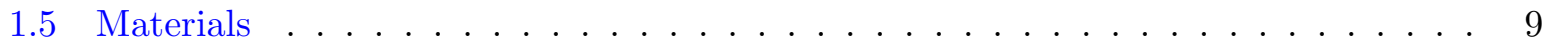

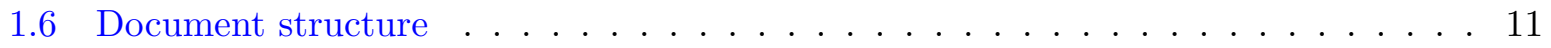

2 Software development: pyColourimetry $\quad 15$

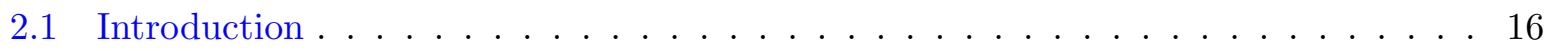

2.2 Colorimetric hardware/software vs open source tools . . . . . . . . . . . . . 18

2.3 pyColourimetry Modules . . . . . . . . . . . . . . . . . . . 22

2.3 .1 Graphical User Interface (GUI) . . . . . . . . . . . . . . . . . . . . . 23

2.3.2 Serial port communication . . . . . . . . . . . . . . 25

2.3 .3 Colorimetric data treatment . . . . . . . . . . . . . . . . 26

2.4 Camera characterization procedure . . . . . . . . . . . . . 28

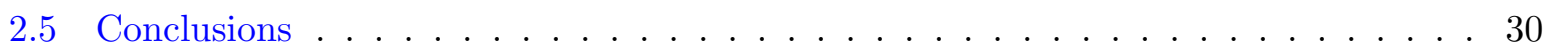

3 Image-based camera characterization $\quad 31$

3.1 Introduction . . . . . . . . . . . . . . . . . . . . . 32

3.2 CIE Color spaces . . . . . . . . . . . . . . . . . . . . . 34

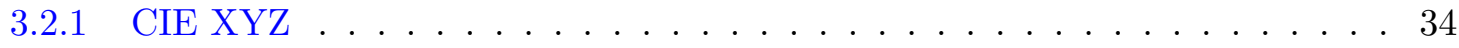

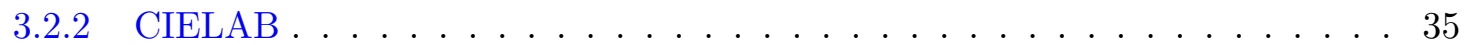

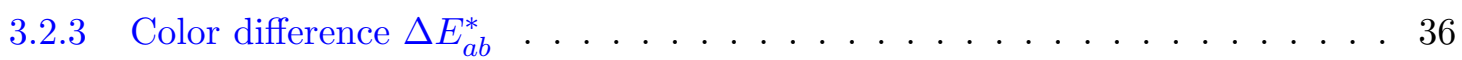

3.3 Material and Methods . . . . . . . . . . . . . . . . 37

3.3 .1 Image data . . . . . . . . . . . . . . . . . . . . 37

3.3 .2 Data processing workflow . . . . . . . . . . . . 38

3.3.3 First stage: Characterization of the digital camera . . . . . . . . . . . . 39

3.3.4 Second stage: Transformation to sRGB space . . . . . . . . . . . . . . 40

3.3.5 RAW data processing . . . . . . . . . . . . . . . . . 41 


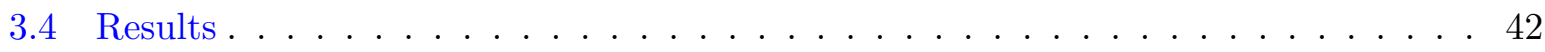

3.4 .1 TIFF data results . . . . . . . . . . . . . . . . . . . 43

3.4 .2 RAW data results . . . . . . . . . . . . . . . . . . . 45

3.5 Discussion . . . . . . . . . . . . . . . . . . . . 47

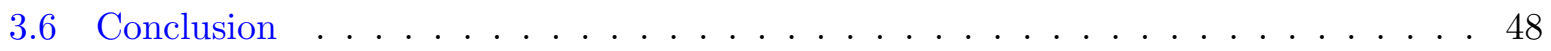

4 A Gaussian Process Model for Camera Characterization $\quad 51$

4.1 Introduction . . . . . . . . . . . . . . . . . . . . . . . . 52

4.2 Materials and Methods . . . . . . . . . . . . . . . . . 53

4.2 .1 Case Study: Cova dels Cavalls . . . . . . . . . . . . . . . . . 53

4.2.2 Image-Based Camera Characterization Methodology . . . . . . . . . . . . 53

4.2.3 Gaussian Processes for Camera Characterization . . . . . . . . . . . . . 55

4.2.4 Second-Order Polynomial Model . . . . . . . . . . . . . . . . . . 57

4.2 .5 Model Checking and Comparison . . . . . . . . . . . . . . . 59

4.2 .6 Induced Noise Analysis . . . . . . . . . . . . . . . . . . . . 59

4.3 Results and Discussion . . . . . . . . . . . . . . . . 60

4.3.1 Model Performance Assessment . . . . . . . . . . . . . . . . . . 60

4.3 .2 Output sRGB Characterized Images . . . . . . . . . . . . . . . . 68

$4.3 .3 \Delta E_{a b}^{*}$ Mapping Images . . . . . . . . . . . . . . . . 68

4.3 .4 Rock Art Specimen Detail Images . . . . . . . . . . . . . . . . 70

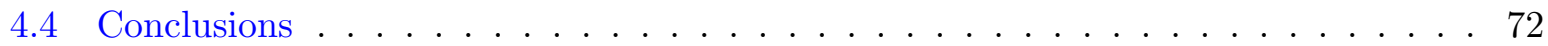

5 Patch Adaptive Selection with $K$-Means (P-ASK) 73

5.1 Introduction . . . . . . . . . . . . . . . . . 75

5.2 Materials and Methods . . . . . . . . . . . . . . . . . . 76

5.2 .1 Data set acquisition . . . . . . . . . . . . . 76

5.2 .2 Colorimetric camera characterization procedure . . . . . . . . . . . . . 77

$5.2 .3 \quad K$-means clustering technique . . . . . . . . . . . . . . . . . 79

5.2.4 Color patch adaptive selection with $K$-means $(\mathrm{P}$-ASK) $\ldots \ldots \ldots$. . . . . 81

5.3 Results . . . . . . . . . . . . . . . . . . . . 84

5.3.1 Setting the optimal parameters for the $K$-means clustering algorithm . . . 84

5.3 .2 Test on the DigiEye imaging system _ . . . . . . . . . . . . . 89

5.3.3 Assessment of the P-ASK framework on rock art images . . . . . . . . . . . 90

5.3.4 Model accuracy assessment of the image-based characterization . . . . . . . 92

5.3.5 Output sRGB characterized images . . . . . . . . . . . . . . . 93

5.4 Discussion . . . . . . . . . . . . . . . . . . . . . . 95

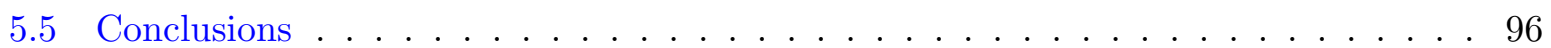


6 Discussion $\quad 97$

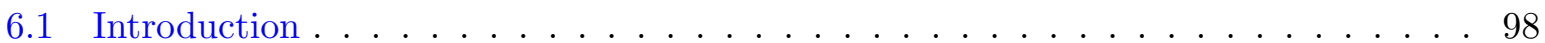

6.2 D65 Illuminant hypothesis . . . . . . . . . . . . . . . . . . . . . 98

6.3 RAW vs processed image files . . . . . . . . . . . . . . . . . . . 101

6.4 Regression model . . . . . . . . . . . . . . . . . . . . . . 104

6.5 Negative CIE XYZ predicted values . . . . . . . . . . . . . 107

6.6 Sample selection . . . . . . . . . . . . . . . . . . . . . 110

6.7 Spectral recovery . . . . . . . . . . . . . . . . . . . . 115

7 Conclusions $\quad 119$

7.1 Initial objectives set and questions raised . . . . . . . . . . . . . 120

7.1.1 Question: Is the camera characterization a suitable procedure for cultural heritage documentation and preservation tasks? . . . . . . . . . . . . 120

7.1.2 Question: Could the GP model provide better results as a regression model for camera characterization? . . . . . . . . . . . . . . . . . 121

7.1.3 Question: Is the full set of chips from the color checker needed for model learning in the characterization procedure? . . . . . . . . . . . . . 121

7.2 Key findings and research contribution . . . . . . . . . . . . . . . . . . 122

7.3 Remaining research challenges . . . . . . . . . . . . . . . . . . . . 124 



\section{List of Figures}

1.1 Rock art cave sites location (Source: $(\mathrm{ICV}, 2020)$ ) . . . . . . . . . . . . . 9

1.2 Rock art scenes: (a,b) Cova dels Cavalls; (c,d) Cova Civil; (a) Canon EOS-1D; (b) Nikon D40; (c) Fujifilm IS PRO; (d) Sigma SD15. . . . . . . . . . . . . . . . 9

1.3 Color Checkers: (a) X-rite ColorChecker@Digital SD chart; (b) X-rite Passport; (c) GretaghMacbeth; (d) Munsell; (e) RAL K-5. . . . . . . . . . . . . . . . 10

1.4 Low-cost light color cabinet. . . . . . . . . . . . . . . . . . . . . . 10

1.5 Relationship between objectives, chapters and papers published. . . . . . . . . 12

2.1 Instrumental and software available: a) CS-100A colorimeter (Konica Minolta, 2020b); b) CS-S10w software (Konica Minolta, 2020c); c) CM-600d spectrophotometer (Konica Minolta, 2020); d) SpectraMagic NX software (Konica Minolta, 2020d) . . . . . . . . . . . . . . . . 20

2.2 Main pyColourimetry modules. . . . . . . . . . . . . . . . . 22

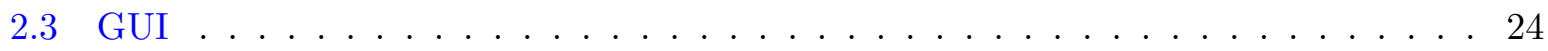

2.4 ToolBar options and their relationship with the project work pipeline . . . . . 24

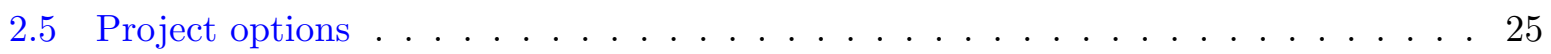

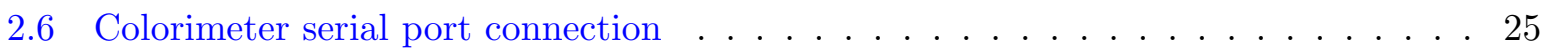

2.7 SerialPort module workflow scheme . . . . . . . . . . . . . 26

2.8 Spectral curve generation example . . . . . . . . . . . . . . . 27

2.9 Basic characterization workflow. . . . . . . . . . . . . 28

2.10 Camera characterization procedure using pyColourimetry software . . . . . . . 28

3.1 Image-based characterization workflow. . . . . . . . . . . . . . . 33

3.2 Example of data acquisition on site. Partial view of the Shelter V, Cova Remigia. . 38

3.3 Measuring RGB data with pyColourimetry software. . . . . . . . . . . . . 38

3.4 Flow chart to show the characterization implemented for digital cameras. . . . . . 39

3.5 Comparative characterization workflow for RAW and TIFF images. . . . . . . . . . 42

3.6 Adjustment results for the characterization of the digital camera (pyColourimetry). 42

3.7 Residuals in the CIE XYZ space with TIFF data (1st, 2nd and 3rd order): a) X; b) $\mathrm{Y}$; c) Z. . . . . . . . . . . . . . . . . . . . . . . . 43

$3.8 \Delta E_{a b}^{*}$ CIELAB color differences with TIFF data. . . . . . . . . . . . 44 
3.9 Specimens selected in the scene: a) wild board; b) hunter; c) nest; and d) layout

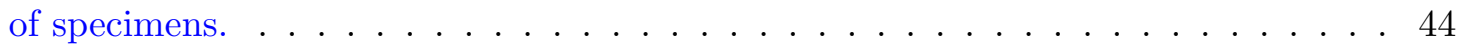

3.10 sRGB output images: a, b, c) Animal detail; d, e, f) Hunter detail; a, d) 1st order; b, e) 2nd order; c, f) 3rd order. . . . . . . . . . . . . . . . . . 45

3.11 Residuals in the CIE XYZ space with RAW data (1st order; 2nd order; 3rd order): a) X; b) Y; c) Z. . . . . . . . . . . . . . . . . . . 46

$3.12 \Delta E_{a b}^{*}$ CIELAB color differences with RAW data . . . . . . . . . . . 47

3.13 sRGB output images of the nest detail: a, b, c ) TIFF data; d, e, f ) RAW data; a, d) 1 st order; b, e) 2nd order; c, f) 3rd order. . . . . . . . . . . . . . . . 47

4.1 Schematic diagram designed for the camera characterization. . . . . . . . . . 53

4.2 RAW images versus processed images workflow. . . . . . . . . . . . . . . 54

4.3 CIE XYZ residuals histograms after the adjustment: (a,b,e,f) Gaussian process; (a,d,g,h); Second-order polynomial; (a,c,e,g); CIE XYZ residuals; (b,d,f,h); LOOCV residuals. . . . . . . . . . . . . . . . . . . . . 61

4.4 Sigma SD15 $\Delta E_{a b}^{*}$ values for the X-Rite patches: (a) $\Delta E_{a b}^{*}$; (b) LOOCV $\Delta E_{a b}^{*}$. . . 63

4.5 Fujifilm IS PRO $\Delta E_{a b}^{*}$ values for the X-Rite patches: (a) $\Delta E_{a b}^{*}$; (b) LOOCV $\Delta E_{a b}^{*}$. 63

4.6 Patches with higher LOOCV $\Delta E_{a b}^{*}$ values: (a) on X-Rite ColorChecker; (b) on CIE chromaticity diagram. . . . . . . . . . . . . . . . . 65

4.7 Noise comparative detail sRGB images after characterization: (a-d) Sigma SD15; (e-h) FujifilmIS PRO; (a,e) Original RAW images; (b,f) GP; (c,g) Second-order polynomial model; $(\mathrm{d}, \mathrm{h})$ Linear model. . . . . . . . . . . . . . . 68

4.8 Original and output sRGB characterized images: (a,c,e) Sigma SD15; (b,d,f) Fujifilm IS PRO; (a,b) Original; (c,d) GP; (e,f) Second-order polynomial model. 69

$4.9 \Delta E_{a b}^{*}$ difference mapping images between GP and second-order polynomial characterization models: (a) Sigma SD15; (b) Fujifilm IS PRO. . . . . . . . . . . . 69

4.10 Selected rock art scenes: (a) SigmaSD15; (b) Fujifilm IS PRO. (A) Wounded animal detail. (B) Hunting scene. . . . . . . . . . . . . . . . . . . . . . . 70

4.11 Wounded animal images: (a-d) Sigma SD15; (e-h) Fujifilm IS PRO; (a,e) Original image; (b,f) GP characterized image; (c,g) $\Delta E_{a b}^{*}$ comparative image; (d,h) Second-order characterized image. . . . . . . . . . . . . . . . . . . 71

4.12 Hunter scene images: (a-d) Sigma SD15; (e-h) Fujifilm IS PRO; (a,e) Original image; (b,f) GP characterized image; $(\mathrm{c}, \mathrm{g}) \Delta E_{a b}^{*}$ comparative image; (d,h) Second-order characterized image. . . . . . . . . . . . . . . . . . 71

5.1 Rock art images: (a) Fujifilm IS PRO (b) Sigma SD15 (c) Nikon D40. . . . . . . . 77

5.2 Colorimetric camera characterization framework . . . . . . . . . . . . 78

5.3 P-ASK framework. . . . . . . . . . . . . . . . . . . . 82

5.4 Support and pigment samples: (a) Nikon D40 image (b) Sigma SD15 image (c) Fujifilm IS PRO image. . . . . . . . . . . . . . . . . . . . . . 83 


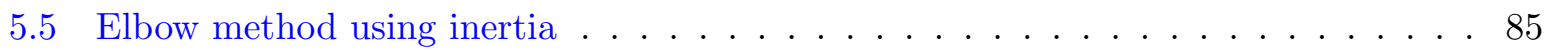

5.6 Silhouette analysis for the Fujifilm IS PRO . . . . . . . . . . . . 86

5.7 Randomness assessment: (a) $\Delta E_{a b}^{*}$ color differences (b) Standard deviation error .87

5.8 Graphic results for the SS method: (a) Patches identified; (b) Processing time. . . 89

5.9 DigiEye imaging system: (a) DigiEye system (without camera integrated) (b) Nikon D40 test image (D65 illuminant). . . . . . . . . . . . . . . . . . 89

5.10 P-ASK framework adapted to the DigiEye D65 image acquired with the Nikon D40. 90

5.11 Graphic description of the pigment sample (P1): (a) clipped detail image, (b) $K$-means centroids, (c) Dominant color palette, (d) Dominant color frequency histogram. . . . . . . . . . . . . . . . . . . . 91

5.12 Color patches detected: (a) Support samples; (b) Pigment samples; (c) Support-pigment; (d) KDP training samples. . . . . . . . . . . . . . . . . 92

5.13 LOOCV CIE XYZ Residuals histogram: (a) FCC (24 patches) (b) KDP (24 patches). 93

$5.14 \Delta E_{a b}^{*}$ color differences: (a) FCC (24 patches); (b) KDP (24 patches). . . . . . . . . 93

5.15 Output sRGB characterized images: (a) FCC training data (96 patches) (b) KDP training data $(24$ patches $) \ldots \ldots \ldots \ldots$. . . . . . . . . . . . . . 94

$5.16 \Delta E_{a b}^{*}$ mapping image $(96-24$ patches $) \ldots \ldots \ldots \ldots \ldots \ldots$

6.1 Original RAW images (displayed as JPEG): (a) normal exposure; (b) overexposed; (c) underexposed. . . . . . . . . . . . . . . . . . . . . . 99

6.2 sRGB output images: (a) normal exposure; (b) overexposed; (c) underexposed. . . 100

6.3 Characterized images: (a,b,c) From JPEG input images; (d,e,f) From RAW input images; (a,d) Linear; (b,e) 2nd; (3,f) 3rd. order . . . . . . . . . . . . . . . . . 102

6.4 $\Delta E_{a b}^{*}$ mapping images: (a) JPEG 2nd - JPEG 1st; (b) JPEG 2nd - JPEG 3rd; (c) X3F 1st - JPEG 1st; (d) X3F 2nd - JPEG 2nd; (c) X3F 3rd - JPEG 3rd; (f) X3F 2nd - X3F 1st; (g) X3F 2nd - X3F 3rd. . . . . . . . . . . . . . . 103

6.5 CIE XYZ residuals: (a) Linear; (b) 2nd; (c) 3rd; (d) 4th; (e) 5th; (f) 6th. . . . . . 105

6.6 Rock art images: (a) Original RAW (displayed as TIFF); (b,c,d,e,f,g,h) Output characterized images; (b) Linear; (c) 2nd; (d) 3rd; (e) 4th. ; (f) 5th.; (g) 6th.; (h)

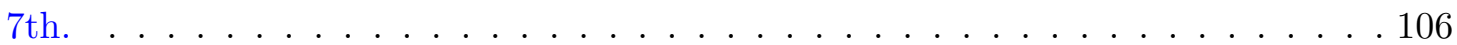

$6.7 \Delta E_{a b}^{*}$ mapping images: (a) X3F 2nd - X3F 1st; (b) X3F 2nd - X3F 3rd; (c) X3F 2nd - X3F 4th; (d) X3F 2nd - X3F 5th; (e) X3F 2nd - X3F 6th; (f) X3F 2nd -

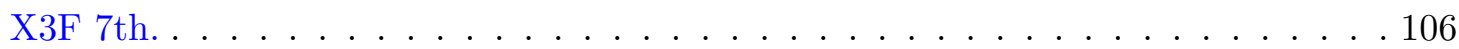

6.8 Non-negativity constraint scheme for camera characterization procedure. . . . . . 108

6.9 Rock art scene images: (a) Original RAW image (displayed as TIFF) ; (b) Output sRGB image; (c) Output sRGB image with the non-negativity constraint. . . . . . 108

6.10 RGB negative predicted values scatter plot. . . . . . . . . . . . . . . 109

6.11 CIE XYZ negative predicted values. . . . . . . . . . . . . . . 109

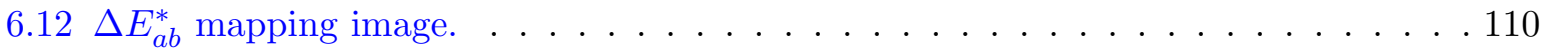

$6.13 \Delta E_{a b}^{*}$ : (a) Mean random model; (b) Best random model. . . . . . . . . . . . . . 111 
6.14 'Skin-tone' dataset patches on CIE chromaticity diagram. . . . . . . . . . . . 113

6.15 Rock art images: (a) Original Sigma SD15 RAW (viewed as TIFF); (b) ColorChecker [140 patches] characterized image; (c) 'Skin-tone' dataset [31 patches] characterized image. . . . . . . . . . . . . . . . . . . 113

$6.16 \Delta E_{a b}^{*}$ mapping images: (a) ColorChecker [140 patches] vs 'Skin-tone' [17 patches]; (b) ColorChecker vs 'Skin-tone' [28 patches]; (c) ColorChecker vs 'Skin-tone' [31 patches]. . . . . . . . . . . . . . . . . . . . . . . . . . 114

6.17 Channel spectral sensitivities: (a) Sigma SD15; (b) Fujifilm IS PRO . . . . . . . 116

6.18 sRGB output images: (a,b,c) Sigma SD15; (d,e,f) Fujifilm IS PRO; (a,d) Second-order polynomial model; (b,e) GP; (c,d) Spectral recovery. . . . . . . . . 117

$6.19 \Delta E_{a b}^{*}$ color difference mapping images: (a,b) Sigma SD15; (c,d) Fujifilm IS PRO; (a,c) Spectral recovery - Second-order polynomial model; (b,d) Spectral recovery - GP model. . . . . . . . . . . . . . . . . . . . . . . . . 117 


\section{List of Tables}

1.1 SLR Technical specifications . . . . . . . . . . . . . . . . . 10

2.1 Manufacturer and colorimetric instruments . . . . . . . . . . . . . . . 19

2.2 Python packages for RAW image processing. . . . . . . . . . . . . . . 21

2.3 Main packages used in pyColourimetry. . . . . . . . . . . . . . . . . 23

3.1 Mathematical models used for camera characterization procedure. . . . . . . . . . 33

3.2 Transformation coefficients matrix (first, second and third order). . . . . . . . . . 40

3.3 Comparative adjustment standard deviation results for the characterization of the digital camera with TIFF and RAW data. . . . . . . . . . . . . . . . . 45

4.1 Fitted CIE XYZ (Res) and LOOCV residuals (RL) values after the characterization. 60

$4.2 \Delta E_{a b}^{*}$ summary of the statistical results after the characterization. . . . . . . . 62

4.3 Percentage of patches with LOOCV $\Delta E_{a b}^{*}>4$ CIELAB units. . . . . . . . . . 64

4.4 Noise variation coefficients of the original RGB digital numbers. . . . . . . . . . . 66

4.5 Variation coefficients of the output CIE XYZ coordinates. . . . . . . . . . . . 66

4.6 Variation coefficients of the output sRGB digital numbers. . . . . . . . . . . . . . 67

4.7 Summary of the variation coefficients obtained. . . . . . . . . . . . . . 67

5.1 Evaluation of $K$-means running time with different values of $K \ldots \ldots$. . . 84

5.2 Comparison of four methods to identify dominant color patches . . . . . . . . . 88

5.3 Assessment of the SS method for the identification of dominant patches . . . . . 88

5.4 Color Patches detected from the Fujifilm IS PRO samples . . . . . . . . . . . . . 91

5.5 CIE XYZ Residuals of the FCC and KDP models . . . . . . . . . . . . . . . 92

$5.6 \Delta E_{a b}^{*}$ color differences in the FCC and KDP characterization experiments . . . . 93

6.1 Model fit assessment . . . . . . . . . . . . . . . . . . . . 100

$6.2 \Delta E_{a b}^{*}$ values for the different polynomial models . . . . . . . . . . . . . . 105

6.3 CIE XYZ residuals and $\Delta E_{a b}^{*}$ after camera characterization. . . . . . . . . 112 

1

\section{Introduction}

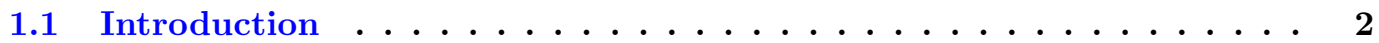

1.2 Background and research justification $\ldots \ldots \ldots \ldots \ldots$

1.3 Objectives ...................... 6

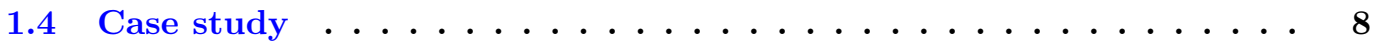

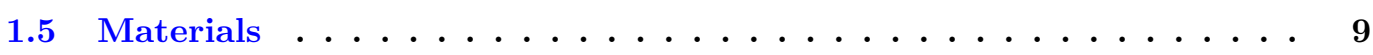

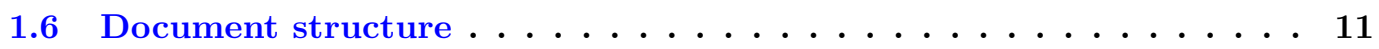




\section{$1.1 \quad$ Introduction}

Cultural heritage documentation and preservation tasks are a complex process which must be addressed with the utmost rigor, since they are considered not only a fundamental tool for the description and conservation of historical assets, but for the analysis, research and dissemination of knowledge. Thus, it is essential to provide effective solutions that provide accurate and contrasted information in cultural heritage documentation applications.

We have to take into account that the risks that endanger the cultural heritage sites have increased alarmingly during the last decades. In addition to erosion, adverse weather conditions or climate change effects, we have to consider the humans' acts of vandalism or terrorism that threaten the integrity of cultural heritage sites (Sesana et al., 2018, Cerra et al., 2016). Particularly vulnerable are those heritage items located in open-air emplacements such as prehistorical rock art painting shelters. Thus, it is not surprising that the heads of cultural heritage conservation agencies, the scientific community and society in general, are especially sensitive about the potential factors that endanger our heritage places.

Exhaustive and complete documentation is essential to adopt concrete actions to preserve the heritage assets, especially those under threat. In order to have accurate data, the integration of novel and improved methodologies as support of common classical techniques used for cultural heritage documentation is required. In this regard, the application of geomatics techniques such as photogrammetry provides accurate 3D data which allows the creation of photorealistic 3D in an easier and faster way. Some examples are 3D photogrammetric and laser scanning documentation (Lerma et al., 2010, Previtali and Valente, 2019, Pan et al., 2019); augmented reality (Barrile et al., 2019, Blanco-Pons et al., 2019); automatic change detection techniques (Cerra et al., 2016); monitoring for control deformation (Tang et al., 2016); microgravimetric surveying technique (Padín et al., 2012); imaging analysis (Maietti et al., 2018); or enhancement methods (Cabrelles et al., 2018, Ippolitiand and Calvano, 2019) to name just a few.

Although the introduction of digital technologies has been a breakthrough for cultural heritage documentation and preservation tasks, there are still aspects that have not been sufficiently addressed. We refer specifically to the accurate color measurement. Undoubtedly, the correct color specification is an essential aspect in archaeology and particularly in prehistorical rock art documentation (Ruiz and Pereira, 2014, Korytkowski and Olejnik-Krugly, 2017). To have accurate color data allows researchers not only the current state description of historical objects, but the study and the diagnosis of its degradation over time (del Hoyo et al., 2015, 2019). However, the color is a physiological and psychophysical phenomenon that occurs in our visual system and brain (Johnson and Fairchild, 2003). Thus, this makes the color a highly subjective attribute. Hence the use of rigorous and non-subjective techniques for the correct measurement of color is required.

The main goal of this thesis is to establish a rigorous colorimetric framework through the characterization of digital cameras for color measurement from images, and their application in rock art documentation and preservation tasks. In addition, camera characterization can be used 
in combination with other photogrammetric techniques for the generation of $3 \mathrm{D}$ colorimetric models as close to reality as possible in cultural heritage applications.

Also, as an additional and fundamental aspect of this research, the proposed methodology has been implemented into a specific open source software, independent of commercial packages, called pyColourimetry. This software allows the treatment of colorimetric and spectral data, and most importantly, the camera characterization for the correct measurement of color. Indeed, it is a pioneering software in its field, since there is a lack of software focused on the colorimetric characterization of digital cameras.

We would like to note that this research was conducted within the scope of the project "Spectrophotometric analysis and calibration of cameras applied to rock art studies" (HAR2014-59873-R), of the Spanish Ministerio de Economía y Competitividad, and partly funded throughout the Research and Development Aid Program PAID-01-16 of the Universitat Politècnica de València.

\subsection{Background and research justification}

The precise recording of color is a priority, yet not trivial, aspect in cultural heritage documentation (Boochs et al., 2014, Korytkowski and Olejnik-Krugly, 2017, Gaiani et al., 2017). Traditional methodologies for color description in archaeology are mostly based on visual procedures usually by means of color chip collections such as the Munsell charts or other commercial color charts designed specifically for photographic applications. However, the drawback of this approach is that color vision largely depends on the experience of the observer, and therefore it is ultimately a matter of perception and strictly subjective. Thereby, a color description based only on visual experiments can considerably affect the results obtained.

To obtain accurate color data it is necessary to rely on non-subjective and rigorous methodologies based on colorimetric criteria, which can be used in combination with more innovative techniques already implemented. Correct color registration requires objective colorimetric measurement in rigorous color spaces, using colorimeters or spectrophotometers. Usually, the color spaces defined by the Commission Internationale de l'Éclairage (CIE) are widely used as the standard reference framework for colorimetric measurement and management (CIE, 2018). However, to avoid any damage to the pigment, direct contact measurements on painted rock art panels are not allowed. Instead, indirect and non-invasive methods for color determination are required.

The use of digitization techniques with conventional digital cameras to support rock art documentation is becoming more and more frequent since the color information obtained from digital images can be easily captured, stored, and processed (González et al., 2009, Rogerio-Candelera, 2015, Robert et al., 2016, Fernández-Lozano et al., 2017, López-Menchero et al., 2017). Thus, the combined use of digital image processing techniques, multispectral analysis and precise photogrammetric techniques have largely benefited the cultural heritage documentation processes. The application of these digital recording methods and imagery analysis 
software together with novel technologies offer great advantages over traditional techniques. The acquisition of complete and high-quality digital data is currently possible in a fast and effective way, reducing the geometric problems associated with classical methods (Yastikli, 2007, Domingo et al., 2015, Lerma et al., 2015).

However, relying on digital images without any color pipeline correction does not provide accurate colorimetric results. Trichromatic digital cameras capture color information in the well-known RGB format. Nevertheless, the drawback of image color data lays on the signal acquired by the built-in sensor, which is not strictly colorimetric. The signals registered by digital cameras are indeed referred to as "device-dependent"; that is, different sensors will record different responses even for the same scene under identical lighting conditions. Also, the RGB data do not satisfy the Luther-Ives condition (Hong et al., 2001, Sharma, 2003). If the values recorded in the RGB channels were proportional to the input energy, a simple linear relationship between the RGB data acquired by the digital camera and the CIE XYZ tristimulus values would exist. However, in general, the RGB data are not a linear combination of CIE XYZ coordinates, and therefore the spectral sensitivities of the three RGB channels are not linear combinations of the color-matching functions (Westland et al., 2012). Thus, a colorimetric procedure is required in order to use the data provided by the digital camera sensor, so that the input RGB data are transformed into device-independent color spaces.

A common approach for accurate color reproduction based on digital images is based on the use of ICC color profiles or camera profiles (Ruiz and Pereira, 2014, Korytkowski and Olejnik-Krugly, 2017). Although the use of ICC color profiles offers good results, it is a closed process that requires the use of specific software. An alternative workflow for correct color registration is the most generic digital camera characterization procedure.

Applications of camera characterization devices can be found in disciplines such as biology (Stevens et al., 2007), medicine (Van Poucke et al., 2010), dentistry (Wee et al., 2007), quality assessment (Girolami et al., 2013), food industry (Leon et al., 2006) and soil science (Kemp, 2014) to name a few. Although the characterization procedure is not a new technique, it has never been applied before in cultural heritage documentation. We are confident that the application of this methodology will contribute significantly to the tasks of correct color specification in heritage documentation.

The purpose of camera characterization procedure is to transform the input device-dependent values captured by the camera sensor, generally in RGB format, into a physically-based, independent color space, such as those defined by the CIE, usually the CIE XYZ color space or any of its derivatives (Martínez-Verdú et al., 2003, Westland et al., 2012, Finlayson et al., 2015). The idea is to obtain quantitative colorimetric information in physically-based color spaces regardless of the device used in the data acquisition.

Different factors can affect the model accuracy such as the built-in camera sensor, the working color spaces, the number of terms for the regression model, the training data size, or the colorimetric properties. These aspects have been analyzed during the development of the thesis.

The image-based camera characterization procedure proposed in this thesis relies on objective 
methods that are independent of the observer experience. It combines the direct method, based on colorimetric measurements obtained using specific instruments (colorimeter and spectrophotometer), and the indirect method, using the RGB values from digital images. We used RAW image files rather than processed or compressed image files since they provide better results in characterization (Westland et al., 2012).

A key aspect to consider is the regression model used in characterization. Different mathematical models have been applied to determine the relationship between the original RGB data and well-defined independent color spaces on the camera characterization procedure. Numerous papers have been written regarding common techniques, such as principal component analysis (Vrhel and Trussell, 1992), interpolation from look-up tables (Hung, 1993), polynomial model (Yoon and Cho, 1999, Hong et al., 2001), artificial neural networks (Cheung and Westland, 2002) or spectral recovery techniques (Cheung et al., 2005, Liang and Wan, 2017). However, the wildly regression model used for the camera characterization is based on polynomial with least-squares fitting with different degrees of variation.

Colorimetric camera characterization procedures based on polynomial regression models have proved to be a suitable approach for the color correction of digital images (Cheung et al., 2004). Thus, we select a polynomial model for camera characterization as a starting point for this research. Afterwards, we will compare results with other alternative mathematical with a different statistical approach, using Bayesian algorithms, with the aim of improving results in the characterization of digital cameras.

Moreover, there is an additional issue to solve which is related to the intrinsic characteristics of rock art shelters. Archaeological caves are generally located in open spaces with difficult access and reduced mobility where the use of specific measuring instruments or portable artificial lighting is unfeasible in most cases. This disadvantage implies that usually, it will not be possible to work under controlled lighting, simulating laboratory conditions during the data acquisition and photographic capture. In controlled lighting conditions, i.e. in the laboratory, the characterization of digital cameras by classical methods obtains satisfactory results. Thus, in rock art applications it will be necessary to evaluate the influence of the lighting conditions of the working environment on the color coordinates.

The digital camera characterization procedure is a suitable methodology for proper color definition in cultural heritage documentation and preservation tasks due to its numerous advantages. Camera characterization is a low-cost and non-invasive technique that allows the acquisition of accurate colorimetric data of complete scenes from digital images in a fast way. After the characterization, once the RGB to CIE XYZ transformation coefficients are obtained, a conventional digital camera can be used for rigorous color specification simulating a colorimeter (Martínez-Verdú et al., 2003). Thus, it is possible to carry out comparisons between different characterized images acquired from different digital devices, since CIE XYZ coordinates are absolute in nature.

Through the camera characterization it is possible to register accurate color data independently of the observer expertise, the camera built-in sensor, the light conditions, or 
the factors involved in the photographic shot. Also, the framework proposed for the camera characterization will be integrated into our own colorimetric software, developed in an open-source manner and independent of commercial software. The aim is to make it available to the scientific community for color research and spread its application in different fields.

In summary, we thought that the research presented in this thesis has a great interest based on the following aspects:

- The obtainment of accurate color measurement from whole image scenes regardless of the sensor device used.

- The development of rigorous software from a colorimetric point of view, with open source modules, will mean new solutions that improve the quality and rigor during the archaeological documentation process, regardless of the lighting and other environmental conditions.

- The proposed characterization framework is a suitable methodology for cultural heritage documentation, as it is a low-cost and non-invasive technique that avoids the subjectivity of the observer.

- The application of characterization to photographic documentation will allow a fully reproducible cataloging procedure of cultural heritage with accurate colorimetric data.

\subsection{Objectives}

The general aim of this thesis is the establishment of a rigorous methodology for the colorimetric characterization of digital cameras, and its application to cultural heritage documentation tasks. The methodological framework proposed will be implemented into a specific software (called pyColourimetry) which will allow the treatment of colorimetric and spectral data.

This research project has its highest aim in the contribution to the protection and preservation of the archaeological heritage, adapting the new digital technologies to optimize the tasks of documenting the cultural heritage, contributing to its greater rigor, particularly concerning the correct recording of color.

The main objective could be split into the following secondary objectives:

- O1. Software development for camera characterization.

- O2. Camera characterization procedure. Application of basic and advanced colorimetry concepts for the digital camera characterization in rock art scenes; color adaptation and standardization.

- O3. Regression model improvement. Establishment of advanced regression models based on Gaussian processes (GP) for model optimization.

- O4. Automated sample selection. Training samples selection for camera characterization, adapted to color range in rock art scenes based on clustering techniques. Set the basis for the development of a specific color chart to archaeological applications. 
Questions to be answered:

- Is the camera characterization a suitable procedure for cultural heritage documentation and preservation tasks?

- Could the GP model provide better results as a regression model for camera characterization?

- Is the full set of chips from the color checker needed for model learning in the characterization procedure?

Hypotheses:

- Classical methodologies for color specification in archaeology are generally based on subjective procedures, depending on the observer experience. Objective methods for color measurements are needed.

- Integration of novel methodologies and procedures from different disciplines for accurate color description.

- The use of digital imaging techniques is currently indispensable as a support tool for traditional methods in cultural heritage documentation tasks.

- The application of common tools for the analysis, processing and image enhancement techniques for pigment identification and digital tracings elaboration is not enough to provide accurate color data.

- Digital images without any colorimetric correction pipeline cannot be used for correct color specification.

- Lack of homogeneous light conditions in open archaeological sites. Rock art shelters are usually open site areas where the photographic shot is not taken under controlled illuminant conditions. Also, the use of specific instruments and accessories are not possible in many cases, since work sites have difficult access or are topographically steep.

- Camera characterization procedure was formulated and tested under laboratory conditions, but not under real rock art work environment scenarios.

Milestones / Activities:

- Learning and testing of basic colorimetry concepts: CIE color spaces, transformation equations, illuminant and standard observers.

- Establishment of a rigorous methodological proposal for the digital camera characterization and their application in rock art scenes.

- RAW image data processing.

- Development of open-source software for colorimetric and spectral data processing. Integration of the methodological process for the characterization of digital cameras.

- Comparison and optimization of the mathematical model based on polynomial regression.

- Testing by means of controlled laboratory/field environmental conditions. 
- Optimization of the mathematical model for the characterization of sensors. Application of Gaussian processes.

- Automatic selection of training samples adapted to the chromatic range of the scene based on clustering techniques.

- Introduction to advanced colorimetry. Bibliographical compilation. Analysis of chromatic adaptation models (CATs). Color standardization.

- Development of Python modules for color adaptation transforms (CATs).

- Optimization and integration of new functionalities in pyColourimetry: Object-oriented programming (OOP), development of new modules, application of the methodological improvements obtained.

- Analysis of the training sample size. Automatic selection of training samples adapted to the color range of the scene. Analysis and publication of results.

- Process automation. Optimization of the modules developed in Python. Application on images taken in the laboratory under controlled lighting conditions, and rock art scenes.

- Construction of a home-made light cabinet for color assessment and spectral recovery techniques. Application in rock art scenarios.

- Publication of results in JCR impact journals, communications in congresses (preferably international with anonymous peer review), attendance to congresses, dissemination and generation of open-access documentation.

\subsection{Case study}

The camera characterization framework proposed will be applied on a set of digital images captured in archaeological rock art sites which are considered part of the 1998 UNESCO World Heritage list (UNESCO, 1998): the "Cova Remigia", "Cova Civil" and "Cova dels Cavalls". These shelters are one of the most singular rock art caves and a well-known reference of the Mediterranean Basin in the Iberian Peninsula. It is part of the Valltorta complex, located in Tírig, Castellón, in eastern Spain (Figure 1.1).

The images correspond to the main representative hunting scenes from these shelters. These graphically narrative scenes make it a unique space in Levantine rock art. Figure 1.2 shows some pictures targeting Levantine rock art motifs taken from these shelters using different Single Lens Reflex (SLR) digital cameras (Source for aerial views (Valltorta, 2013)). Undoubtedly, these are vivid and spectacular human-centered scenes, filled with movement, where the use of reddish pigment predominates (Sarriá Boscovich, 1988, Martínez and Villaverde, 2002).

In our application research field, only a few colors are representative of the pigments and the support in cave painting. In fact, the recording of Levantine rock art archaeological shelters requires a very specific color domain, mostly in the chromatic range of red, brown, and yellow colors. We are focused on the accurate description of pigments particularly in this chromatic range by means of camera characterization. 


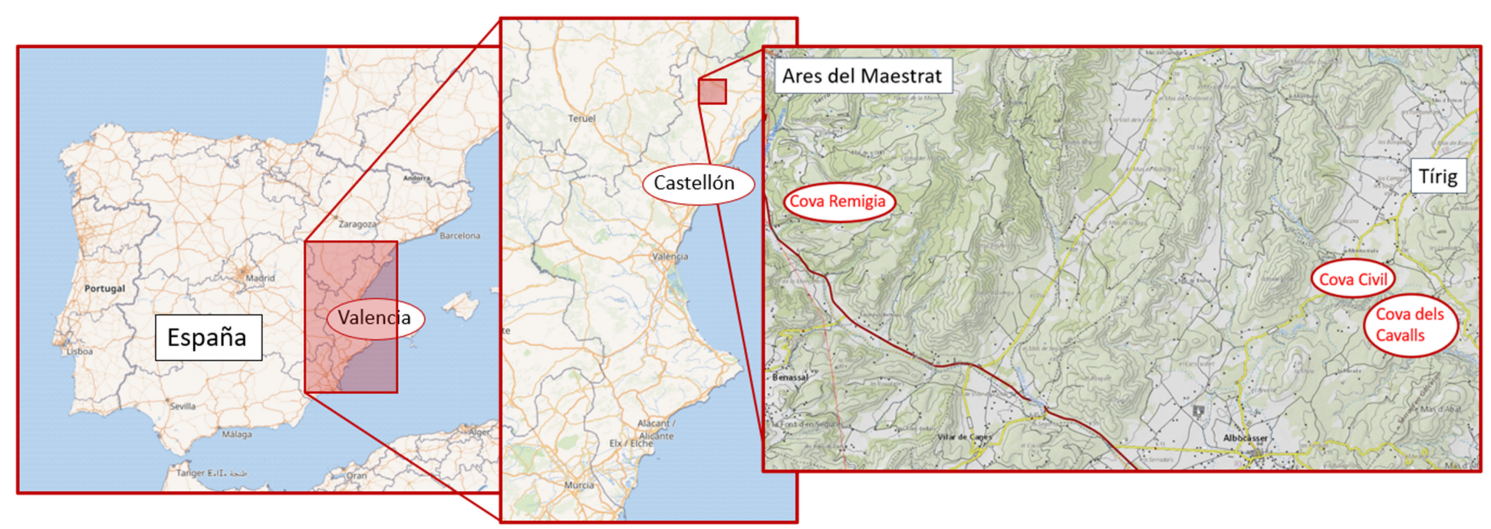

Figure 1.1: Rock art cave sites location (Source: (ICV, 2020))
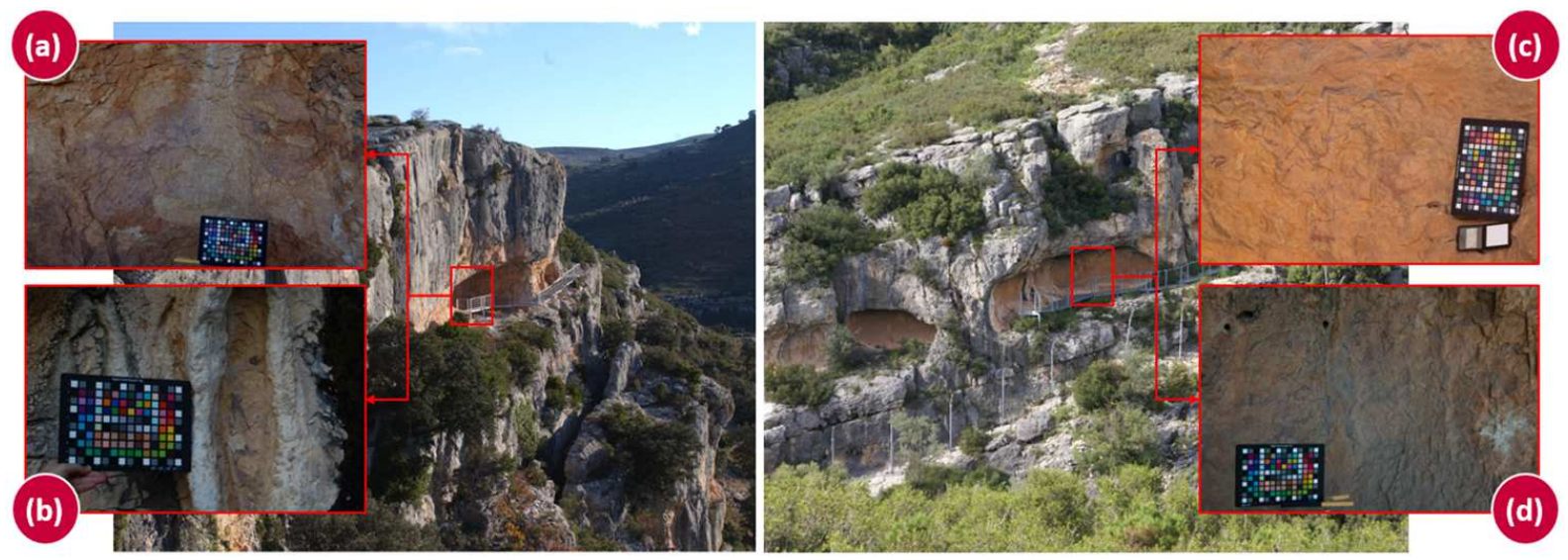

Figure 1.2: Rock art scenes: (a,b) Cova dels Cavalls; (c,d) Cova Civil; (a) Canon EOS-1D; (b) Nikon D40; (c) Fujifilm IS PRO; (d) Sigma SD15.

\subsection{Materials}

For image acquisition, different SLR cameras were used. All these digital cameras allow taking pictures in a RAW format. We prefer using images in RAW format rather than processed since they offer better results after the characterization procedure. Also, we selected these cameras because they have very different built-in image sensors, in order to evaluate their behaviors during the characterization.

Most of the commercial sensors integrated into digital cameras are monochrome arrays with color filter array (CFA) and Bayern pattern for color interpolation. In this thesis, we included a camera with a direct trichromatic sensor without CFA, which does not require any interpolation steps. Table 1.1 shows the principal technical specifications of the cameras used during this thesis research. 
Table 1.1: SLR Technical specifications

\begin{tabular}{lllll}
\hline SLR & Sensor & Size sensor & Img. res. & MP \\
\hline Fujifilm IS PRO & Super CCD $\left(45^{\circ}\right)$ & $23.0 \times 15.5 \mathrm{~mm}$ & $3004 \times 2030$ & 6.10 \\
Nikon D40 & CCD & $23.7 \times 15.5 \mathrm{~mm}$ & $3008 \times 2000$ & 6.00 \\
Sigma SD15 & CMOS Foveon@ $\mathrm{X} 3$ & $20.7 \times 13.8 \mathrm{~mm}$ & $2640 \times 1760$ & 4.70 \\
Canon EOS-1D & CCD & $27.0 \times 17.8 \mathrm{~mm}$ & $2464 \times 1648$ & 4.48 \\
\hline
\end{tabular}

The digital pictures used in the characterization must contain a color chart as colorimetric reference. In our study, we chose the following set of commercial color checkers (Figure 1.3): X-rite ColorChecker@ Digital SD chart (140 patches); X-rite Passport (24 patches); GretaghMacbeth (24 patches); Munsell (322 patches); and the RAL K-5 (213 patches). The spectral reflectance data of the color patches from the ColorChecker were measured directly using the spectrophotometer Konica Minolta CM-600d, under the CIE recommendations $\left(2^{\circ}\right.$ standard observer, and D65 illuminant).

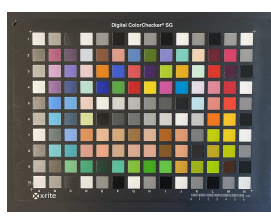

(a)

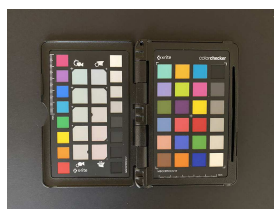

(b)

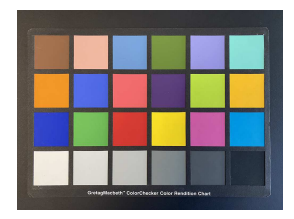

(c)

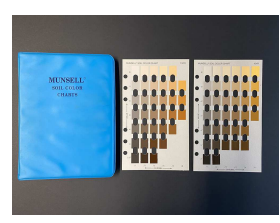

(d)

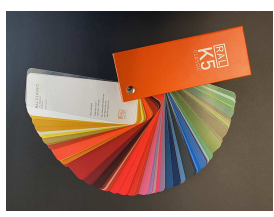

(e)

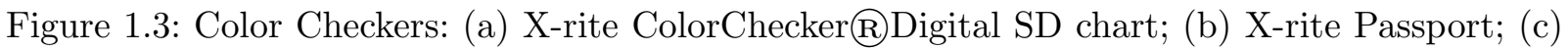
GretaghMacbeth; (d) Munsell; (e) RAL K-5.

As an additional item, the construction of a low-cost color cabinet is planned, which will allow measurements taken under controlled lighting conditions (Figure 1.4). The aim is to conduct spectral recovery experiments. This is a fundamental and novel aspect, since they have not been applied in rock art yet.

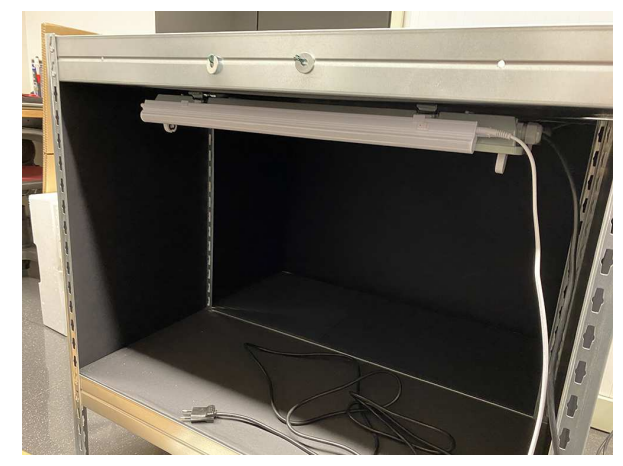

Figure 1.4: Low-cost light color cabinet. 


\subsection{Document structure}

This document is composed of seven chapters, based on the edited versions of three international scientific publications and five published conference papers, which summarize the methodological scheme proposed, the results achieved and its interpretation.

After the introduction, background and research justification exposed in Chapter 1, in Chapter 2 we report on pyColourimetry software that was developed and tested along the lines of the Commission Internationale de l'Éclairage (CIE). This software allows users to control the entire digital image processing and the colorimetric data workflow, including the rigorous processing of RAW data.

Chapter 3 is focused on the establishment of a rigorous camera characterization framework based on polynomial models. We also present its application on Levantine rock art images as a novel field of application. Thus, once the camera is characterized, users obtain output images in the sRGB space that are independent of the sensor of the camera. Three polynomial models were tested for the transformation between color spaces. The outcomes obtained were satisfactory and promising, especially with RAW files. The best results were obtained with a second-order polynomial model, achieving residuals below three CIELAB units. We highlight several factors that must be taken into account, such as the geometry of the shot and the light conditions, which are determining factors for the correct characterization of a digital camera.

In Chapter 4 we propose a novel approach to undertake the colorimetric camera characterization procedure based on a Gaussian process (GP) model, since GPs are powerful and flexible nonparametric models to model multivariate nonlinear functions. To validate the GP model, we compare the results achieved with a second-order polynomial model, which is the most widely used regression model for characterization purposes. Although the output characterized images show that both regression models are suitable for practical applications in cultural heritage documentation, the results show that colorimetric characterization based on the Gaussian process provides significantly better results.

Moreover, in Chapter 5 we introduce a novel framework named the Patch Adaptive Selection with $K$-Means (P-ASK), for the automated selection of color samples for model training within the camera characterization procedure instead of using a whole color checker. We tested the methodology proposed on a set of prehistoric rock art painting images. The results confirm that the characterization approach based on the P-ASK framework allows the reduction of the training sample size and a better color adjustment to the chromatic range of the input scene. Thus, the P-ASK framework reported is a suitable methodology that improves the colorimetric results for camera characterization in cultural heritage documentation and preservation tasks.

Finally, in Chapter 6 we set out the discussion, while in Chapter 7 we summarize the main conclusions, and future research lines based on the results obtained during this research.

Figure 1.5 shows the relationship between the main objectives set in this thesis and the papers published during the research period. 


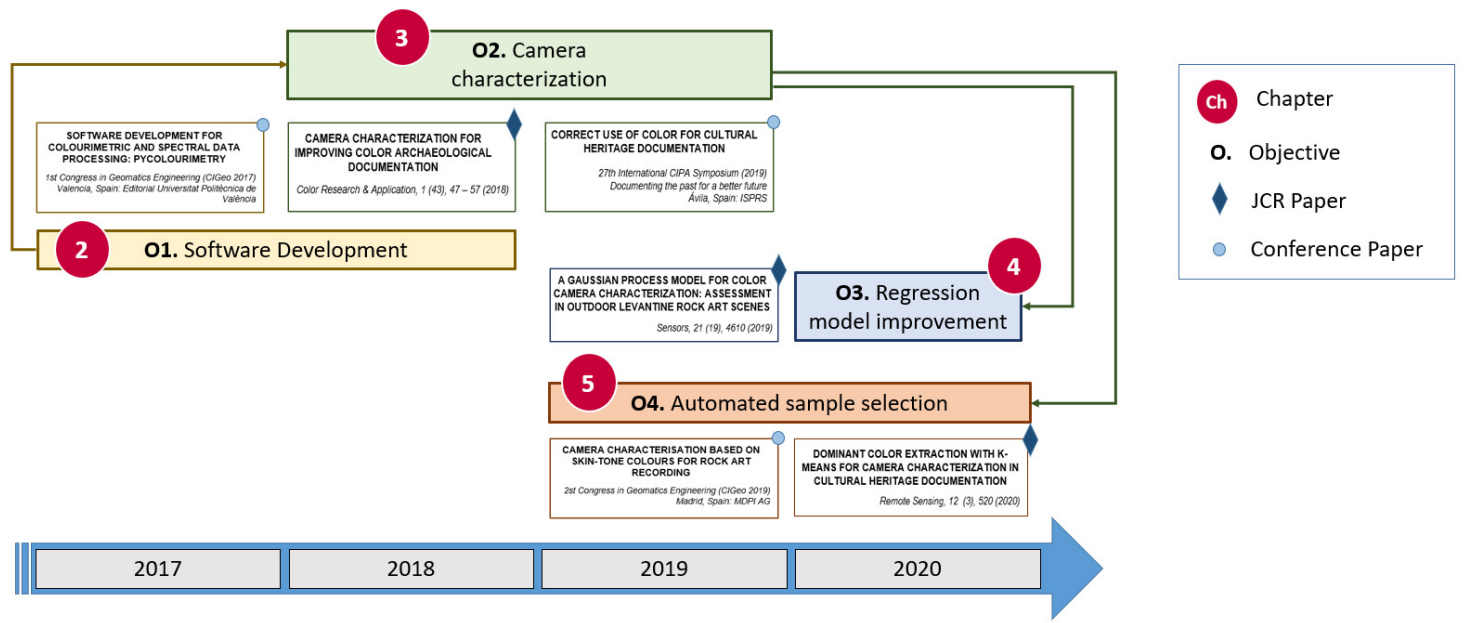

Figure 1.5: Relationship between objectives, chapters and papers published.

We summarize below the papers which have been the basis for the elaboration of each chapter in this document. It should be noted that this document satisfies the guidelines for the presentation of PhD Theses at the Universitat Politècnica de València (Spain) with previously published results.

Chapter 2.

Molada-Tebar, A.; Lerma, J.L.; Marqués-Mateu, Á. Camera characterization for improving color archaeological documentation. Color Res. Appl. 2018, 43, 47-57. [CrossRef]

Molada-Tebar, A.; Lerma, J.L; Marqués-Mateu, Á. Software development for colorimetric and spectral data processing: PyColourimetry. In Proceedings of the 1st Congress in Geomatics Engineering, Valencia, Spain, 5-6 July 2017; Volume 1, 48-53. [CrossRef]

Chapter 3.

Molada-Tebar, A.; Lerma, J.L.; Marqués-Mateu, Á. Camera characterization for improving color archaeological documentation. Color Res. Appl. 2018, 43, 47-57. [CrossRef]

Molada-Tebar, A.; Marqués-Mateu, Á.; Lerma, J.L. Correct use of color for cultural heritage documentation. ISPRS Ann. Photogramm. Remote Sens. Spatial Inf. Sci. 2019, IV-2/W6, 107-113. [CrossRef]

Chapter 4.

Molada-Tebar, A.; Riutort-Mayol, G.; Marqués-Mateu, Á.; Lerma, J.L. A Gaussian Process Model for Color Camera Characterization: Assessment in Outdoor Levantine Rock Art Scenes. Sensors 2019, 19, 4610. [CrossRef] 
Chapter 5 .

Molada-Tebar, A.; Marqués-Mateu, Á.; Lerma, J.L.; Westland, S. Dominant Color Extraction with $K$-Means for Camera Characterization in Cultural Heritage Documentation. Remote Sens. 2020, 12, 520 [CrossRef]

Molada-Tebar, A.; Marqués-Mateu, Á.; Lerma, J.L. Camera Characterisation Based on Skin-Tone Colours for Rock Art Recording. Proceedings 2019, 19, 12. [CrossRef] 

2

\section{Software development: pyColourimetry}

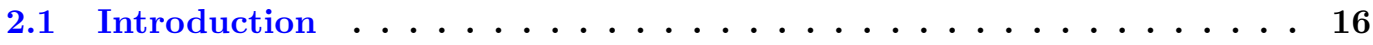

2.2 Colorimetric hardware/software vs open source tools . . . . . . 18

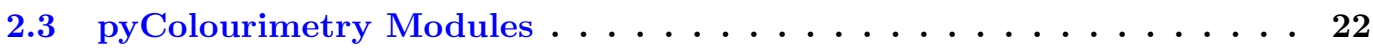

2.3 .1 Graphical User Interface (GUI) . . . . . . . . . . . . . . . . . 23

2.3.2 Serial port communication . . . . . . . . . . . . . . . 25

2.3 .3 Colorimetric data treatment . . . . . . . . . . . . . . . . 26

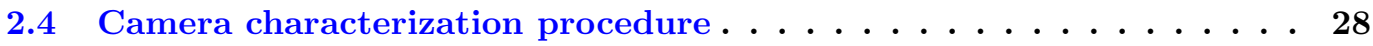

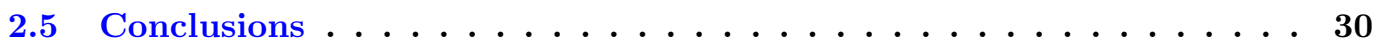




\section{$2.1 \quad$ Introduction}

Archaeological documentation and preservation tasks are complex processes where the technical measurement and specification of color is an essential aspect (Boochs et al., 2014, Korytkowski and Olejnik-Krugly, 2017). The correct color definition allows a more comprehensive graphic definition, a better understanding of the scene, and provides relevant technical information especially for study and preservation work (Ruiz and Pereira, 2014). Nevertheless, classical methodologies for color specification in cultural heritage applications are based mainly on visual observations using a color chart as colorimetric reference. The issue with those methods is that perceptual procedures limits the results since they are strictly subjective. Color is a matter of perception and color vision depends on the observer. Thus, a rigorous colorimetric technique is necessary to properly support rock art research (Ohta and Robertson, 2005).

The ideal setup would allow taking direct measurements using color rigorous instruments such as colorimeters or spectrophotometers. However, in most archaeological practical cases, especially on-site surveys, direct measurements are not viable due to the working environment, or are not allowed to avoid any damage to the pigments. Nowadays, it is essential to support classical methodologies with digital techniques. In fact, in the last years cultural heritage documentation processes have largely benefited from the application of digital recording methods, image analysis software and technologies that offer great advantages over the traditional methods (Boyd et al., 2016, Higuchi et al., 2016). In the particular case of color communication, the drawback is that the signals generated by the digital camera (mostly in RGB format) are device-dependent and not colorimetrically sound. They do not satisfy the Luther-Yves condition, that is, do not directly correspond to the device-independent tristimulus values based on the CIE standard colorimetric observer (Hong et al., 2001, Sharma, 2003).

An approach to transforming RGB dependent values into an independent color space (such CIE XYZ or sRGB which is somehow based on CIE XYZ), is the digital camera characterization by polynomial regression with least-squares fitting (Balasubramanian, 2003, Malacara, 2011, Westland et al., 2012). Once the transformation equations are obtained, a conventional digital camera could be used for a rigorous color determination as if it were a colorimeter (Martínez-Verdú et al., 2003). In Chapter 3 we provide further information about the framework proposed for camera characterization procedure.

However, the rigorous processing of color data requires the implementation of objective methodologies integrated into software packages. In addition, the software must satisfy specific colorimetric technical characteristics, such as the colorimetric reports published by the Commission Internationale de l'Éclairage (CIE). There are different software options, both for the treatment of colorimetric samples, generally provided by the manufacturer of the instrument used in the measurement, as well as for the digital image processing (Bergman et al., 2008). Although in both cases they are, to some extent, versatile software systems with wide functionality, they generally do not allow the user to have absolute control on the methodological process. Moreover, it is not usual to find software that allows processing RAW RGB data, characterizing 
digital cameras, and specially applying characterization parameters on input images to obtain characterized output sRGB images.

Given the importance of color communication in archaeology, and the lack of specific software for camera characterization, we have developed our own software package for the treatment of colorimetric and spectral data. In this chapter we describe pyColourimetry, a software system that was developed and tested taking into account the recommendations of the CIE. Undoubtedly, this is one of the key aspects framed in this thesis not only from a methodological point of view, but also for the time and resources invested in the development of the software.

pyColourimetry was developed in Python (v.2.7) which is an interpreted and multiplatform programming language, released under the GNU general public license, which guarantees end users the freedom to use, study, share and modify the software (Python Software Foundation, 2020). Originally, the computer platform chosen for programming tasks was Linux, specifically the Ubuntu 14.04 Desktop distribution (Canonical Ltd, 2020). However, pyColourimetry works also under the Windows environment (Microsoft, 2020), since it is a multiplatform software that only requires minor changes to the code.

Python has undergone a remarkable increasing growth as programming language in the development of scientific applications (Oliphant, 2007, Chudoba et al., 2013). Some Python development applications are in software engineering, graphical user interfaces, numerical computing, network and internet programming and data analysis and visualization to name a few. However, we are very interested in Python programming since it has proved to be a suitable programming language for geomatics applications such as remote sensing (Clewley et al., 2014), photogrammetry (Barbero-García et al., 2020), spatial management data (Westra, 2013), and GIS tools development (Zambelli et al., 2013, Bunting et al., 2014).

Python emergence during the last decade has made it a potentially competitive open-source alternative to other programming languages such as MATLAB ${ }^{\circledR}$ or Octave (Chaves et al., 2006). Python programming offers several advantages compared with compiled languages. Python is an interpreted, open-source, general-purpose, object-oriented, dynamic and high-level scripting language (Van Rossum and Drake, 2011, Rocha and Ferreira, 2018). Another important aspect of Python is its clean, elegant and simple syntax, easy to read and understand, learn and implement, which in turn significantly reduces the program maintenance cost.

Thus, Python is based on an intuitive and simple read object-based implementation code which allows to easily integrate additional functions. All these attributes make Python a suitable language for programming scientific applications. Nevertheless, Python has an important disadvantage. Python is not recommended for scientific applications which require intensive computations since it runs slower than compiled languages (Cai et al., 2005, Langtangen and Cai, 2008). Programmers have to be particularly careful with long nested loops and complex data structures (Behnel et al., 2011). It is highly recommended to migrate to compiled languages such $\mathrm{C}$ some code parts to settle the lack of efficiency of Python in these cases.

Another important advantage is related to the fact that Python supports the vast amount open source libraries available to programmers. Powerful and optimized scientific packages have 
been developed or ported to cover the needs of users in many research fields. The use of these packages simplifies the computation of complex numerical operations, for instance NumPy (2020) or SciPy (2020), or image processing packages (OpenCV, 2020) to name a few. In fact, it is really easy to find different Python packages which are developed to solve most of the real-world problem programming we have to face in.

All the positive aspects exposed above justify our choice of Python as a programming language for the development of our software. pyColourimetry has as final objective which is to apply a rigorous procedure for the characterization of cameras based on polynomial models. Since we will provide in-depth explanations about the camera characterization procedure in Chapter 3, we present in the following sections a brief description about the methodology proposed, and how it was integrated in our software. We also want to highlight that pyColourimetry allows users to achieve a full control during each one of the phases during the characterization process.

In short, pyColourimetry allows users to apply a procedure for the camera characterization to collect quantitative colorimetric information in physically-based color spaces (McDonald and Rigg, 1997, Malacara, 2011, Ohta and Robertson, 2005). The idea is to achieve color information independently of the device used in the data acquisition stage, relying on digital images and colorimetric measurements only.

\subsection{Colorimetric hardware/software vs open source tools}

In this section, we expose a general background of the resources available about specific colorimetric instrumental and software provided by manufacturers, generally under a commercial license, compared with open-source free tools developed for color management.

Physical color measurement is based on the CIE colorimetric system (Schanda et al., 2007, CIE, 2018). The instruments used in colorimetry are listed into three basic types: colorimeters, spectrophotometers and spectroradiometers. A tristimulus colorimeter is the simplest instrument used for color measurement, designed for psychophysical color analysis since it provides measurements that are correlated with a human eye-brain perception (McDonald and Rigg, 1997). Like the human vision system a colorimeter has red, green and blue photodetectors. Thus, a colorimeter provides the tristimulus values (CIE XYZ) or chromaticity coordinates (xyY) from an object.

On the contrary, spectroradiometers measure spectral quantities of light sources, and spectrophotometers measure spectral reflectance and transmittance of objects. From the spectral reflectance data acquired from spectrophotometers the tristimulus CIE XYZ values can be easily obtained through the color matching functions (CMFs) specified by the CIE (CIE, 2018). Although tristimulus colorimeters are fast, simple, and cheaper compared with spectrophotometers, the data recorded by spectrophotometers are more complete (spectral rather than just three tristimulus values) and accurate. Therefore, for accurate colorimetric measurement rather than a colorimeter the use of a spectrophotometer is highly recommended. Further detailed 
explanation about components, measurement mode, geometry, critical parameters, errors in color measurement are provided in the literature (Ohta and Robertson, 2005b, Ohno, 2007).

Table 2.1 shows the main colorimetric instrument manufacturers, and some of their products available as well. Usually, together with the instrument, the manufacturer provides a limited license software with the basic operations, which allows the connection with a PC, taking direct measures, direct data transfer and management, and colorimetric standard computations (BYK, 2020, Topcon, 2020).

Table 2.1: Manufacturer and colorimetric instruments

\begin{tabular}{|c|c|c|}
\hline Manufacturer & Country & Product \\
\hline Konica Minolta & Japan & $\begin{array}{l}\text { Luminance color meters } \\
\text { Chroma Meters / Colorimeters } \\
\text { Imaging photometers and colorimeters } \\
\text { Portable spectrophotometers }\end{array}$ \\
\hline X-Rite & USA & $\begin{array}{l}\text { Portable spectrophotometers } \\
\text { Imaging Spectrocolorimeter }\end{array}$ \\
\hline Topcon & Japan & Luminance colorimeters \\
\hline BYK & Germany & Portable Spectrophotometers \\
\hline PCE Instruments & Germany & $\begin{array}{l}\text { Colorimeter } \\
\text { Spectrophotometer }\end{array}$ \\
\hline Capital instrument & China & Colorimeters \\
\hline
\end{tabular}

However, there are more powerful packages developed to compute more complex colorimetric operations, or for specific applications such as Colibri@for color appearance (X-Rite, 2020d), CM-SA skin analysis (X-Rite, 2020c) or different software solutions for quality control in industry applications (Color iMatch, InkFormulation Software, ColorCert Suite, Color iQC, ColorDesigner PLUS and NetProfiler (all provided by X-Rite (2020b)). An interesting tool is a mobile app developed by X-Rite called Color-Eye $\mathbb{R}$, which allows consumers to ensure color match between items using a smartphone and a calibration card (X-Rite, 2020e).

Two-color measurement instruments are available for this research: a CS-100A Konica Minolta colorimeter, currently discontinued and replaced by the CS-150 and CS-160 models; and a CM-600d Konica Minolta spectrophotometer (Figure $2.1 \mathrm{a}, \mathrm{c}$ ). The manufacturer usually provides software to connect the instruments to a computer, allowing users the access and management of the colorimetric recorded measurements. The problem is that commonly, the type of commercial license purchased conditions the functions of the software available to users. Full access to software usually implies a substantial increase in equipment cost.

The CS-100A is a portable, non-contact digital tristimulus colorimeter designed for remote measurement of chromaticity coordinates and luminance value. On the other hand, the CM-600d is a portable measurement instrument as well, but in this case designed to register the spectral reflectance of an object, providing more accurate data. Thus, its advanced capabilities make it an optimal instrument to evaluate, reproduce, and control the color of objects more efficiently. 
The CS-100A colorimeter is compatible with the CS-S10w data management software, and the software for the CM-600d spectrophotometer is SpectraMagic NX (Figure 2.1 b, d). We have not used the CS-S10w software, since pyColourimetry is designed to perform direct measurements through the CS-100A colorimeter. However, the colorimetric data from the color checker was taken using the CM-600d spectrophotometer in order to acquire more accurate data. Thus, we used the SpectraMagic NX software only to save and transfer the registered data. The colorimetric data management (such as color space transforms among other operations) were carried out using our own Python scripts, since we have not full license (Pro version) to use the software. As we will discuss below, although commercial software provides powerful functions and a high versatility, it is possible to perform the same operations achieving satisfying results using open source tools.

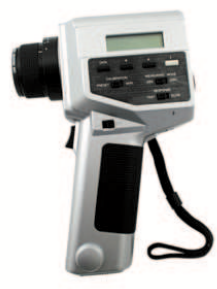

(a)

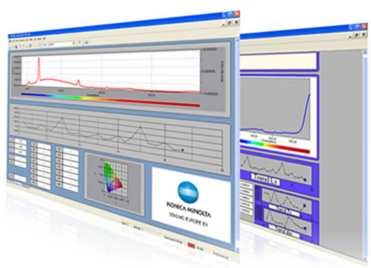

(b)

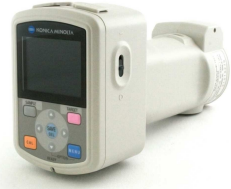

(c)

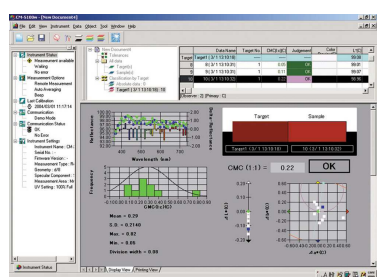

(d)

Figure 2.1: Instrumental and software available: a) CS-100A colorimeter (Konica Minolta, 2020b); b) CS-S10w software (Konica Minolta, 2020c); c) CM-600d spectrophotometer (Konica Minolta, 2020); d) SpectraMagic NX software (Konica Minolta, 2020d)

Regarding digital image processing software, there are different commercial options available. However, probably one of the most powerful and widely used for graphic designers, photographers, illustrators and even scientists is Photoshop (Ps) (Adobe Photoshop, 2020). Ps software is able to read and process RAW images from different camera sensors through the Adobe Camera RAW plugging (Adobe Camera RAW, 2020). An open source alternative for Ps is the multiplatform GIMP image manipulation program (GIMP , 2020). GIMP uses the same method as Ps to read and edit RAW images, that is, it needs to be supported by an additional plugging. A good option is to use RawTherapee, which is an open-source and cross-platform RAW image processing program (RawTherapee, 2020). Although RawTherapee can be used in combination with GIMP, it works well by itself. RawTherapee is a powerful software, with high level tools that parallel those of Ps, but with the advantage of being open-source.

Another open-source suitable option for digital image processing including RAW files processing is ImageMagick, which also provides Python bindings (ImageMagick, 2020). In fact, Python provides several libraries which allow RAW images management, with interesting funcionalities and computations. Table 2.2 shows a few examples of Python packages developed, some of which are based on DCRAW decoding (Coffin, 2020). Thus, using Python packages users can apply similar operations as when using commercial image processing software. 
Table 2.2: Python packages for RAW image processing.

\begin{tabular}{ll}
\hline Package & Source \\
\hline RawPy & https://letmaik.github.io/rawpy/api/rawpy.RawPy.html \\
LibRaw & https://www.libraw.org/ \\
rawkit & https://rawkit.readthedocs.io/en/latest/index.html \\
\hline
\end{tabular}

On the other hand, a suitable and widely approach for accurate color reproduction from digital images is through the application of ICC color profiles or camera profiles (Ruiz and Pereira, 2014, Korytkowski and Olejnik-Krugly, 2017, ICC, 2020). Although the use of ICC color profiles offers good results, it is a closed and complex process that requires the use of specific software for camera profiling. ICC provides a list of existing tools for making, editing and assessing ICC profiles provided by partners such as Adobe, HP and X-Rite (Profiling tools, 2020). Some examples are the X-Rite ColorChecker Camera Calibration Software, i1Publish and the app ColorTRUE (X-Rite, 2020). However, open-source tools for ICC compatible color management system and camera profiles are available, such as ArgyllCMS (ArgyllCMS, 2020), DCamProf (DCamProf, 2020), dcpTool (SOURCEFORGE, 2020).

To finish this section on color software, we want to focus on different tools developed for the computation of colorimetric operations. Specific functions are implemented for MATLAB ${ }^{\circledR}$ programmers, such as color space conversion, ICC profiling and automatic white balance (MathWorks $\AA$, 2020). Additional colorimetric packages developed in MATLAB ${ }^{\circledR}$ are: Computational Colour Science (Westland, 2012), and ColorLab which was developed by the Universitat de València (Malo and Luque, 2002). Moreover, Python users can take advantage of the functions implemented in colorimetric open-source packages such as color (Colour, 2013) and colormath (Colormath, 2014). Additionally, it is not uncommon to find web pages that allow color transformations and other operations to be performed online (ColorMine, 2020, Lindbloom, 2020).

In conclusion, after exposing the different options about the specific colorimetric instrumental and software available, both for colorimetric data management and image processing, it is clear that software which integrates both methodologies is highly recommended. pyColourimetry not only combines both processes, but also uses them to carry out the digital camera characterization in order to yield accurate colorimetric data from full images, particularly in RAW format. Our software covers the main colorimetrical requirements, offers accurate results, and it is actually a pioneering software not only in color management but in its application to rock art painting color documentation for cultural heritage tasks. In the following sections, we provide further details about the pyColourimetry software development. 


\section{3 pyColourimetry Modules}

The computer software pyColourimetry consists of a set of modules that allows users: to acquire direct measurements using a CS-100A colorimeter (serialport.py); process colorimetric and spectral data based on the main CIE colorimetric technical recommendations (myColour.py); obtain the transformation equations for the camera characterization (using polynomial regression models), and apply the equations on the image to create images in the final sRGB space (characterization.py).

These modules are managed by users through a graphic user interface or GUI (gui.py). In Figure 2.2 we present a graphic scheme which shows the interconnection between the developed modules and their main functionalities. Further details about each one of the modules integrated in pyColourimetry are provided in the following subsections.

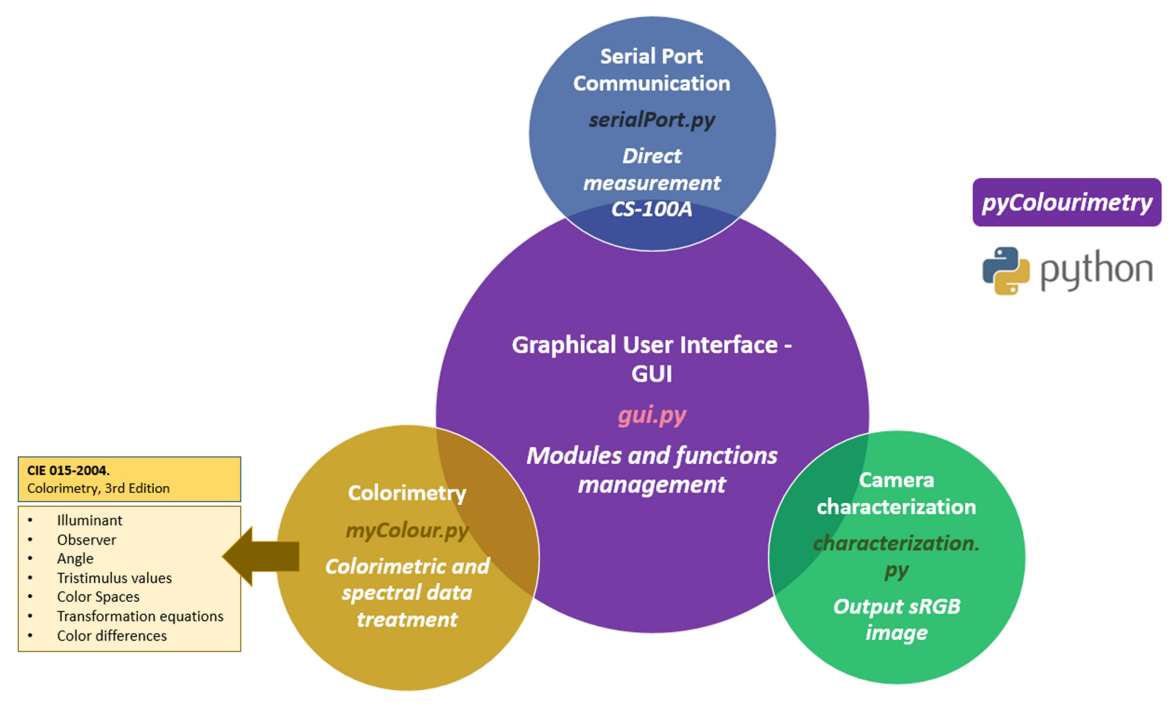

Figure 2.2: Main pyColourimetry modules.

As we discussed in the introduction, the scripts developed in Python can be supported by a vast amount of specific technical libraries available to programmers. Thus, we can feed on powerful functions that have already been created and tested by developers, which simplifies the programming task. Table 2.3 lists the most significant packages used for pyColourimetry development, the source and a brief description of their general application. Among these packages, the following stand out: RawPy for the management of digital images in RAW format; NumPy and SciPy for computing complex operations; and PyQt4 for the design of the GUI. 
Table 2.3: Main packages used in pyColourimetry.

\begin{tabular}{lll}
\hline Package & Source & Application \\
\hline os & https://docs.python.org///ibrary/os.html & Operative system functions \\
shutil & https://docs.python.org/2/library/shutil.html & Operations with files \\
sys & https://docs.python.org/2/library/sys.html & Python functions and attributes \\
glob & https://docs.python.org///ibrary/glob.html & Pathfiles in Unix \\
pySerial & http://pythonhosted.org/pyserial/ & Serial Port communication \\
random & https://docs.python.org/2/library/random.html & Work with random numbers \\
PIL & http://pythonware.com/products/pil/ & Digital images treatment \\
RawPy & https://letmaik.github.io/rawpy/api/rawpy.RawPy.html & Treatment of RAW images \\
math & https://docs.python.org/2/library/math.html & Mathematical functions \\
NumPy & http://www.numpy.org & Matrix calculation \\
SciPy & http://www.scipy.org & Scientific computations \\
matplotlib & http://matplotlib.org & Plot \\
colormath & http://python-colormath.readthedocs.io/en/latest/ & Colorimetric and spectral data treatment \\
PyQt4 & http://pyqt.sourceforge.net/Docs/PyQt4/ & Graphic user interface design \\
\hline
\end{tabular}

\subsubsection{Graphical User Interface (GUI)}

The GUI (gui.py script) is undoubtedly the heart or key piece of pyColourimetry software. The rest of the modules complement the colorimetric functions that allow the user to complete the characterization framework pursued in this thesis. Python has several graphical user interface (GUI) frameworks available. The native Python GUI is the Tkinter (Tk) package (Python Software Foundation, 2020b). However, the pyColourimetry GUI has been designed based on the PyQt4 library funcionalities, which is a multiplatform binding of the Qt graphical library for Python. PyQt provides useful tools to make a simple, but complete GUI. In addition, the GUI has been created with the aim of being intuitive, easy-to-use and attractive for users, with a technical and professional appearance.

Figure 2.3 shows the main objects integrated in the pyColourimetry interface: Menu (basic operations); ToolBar (Fast icon access menu); MainWindow (to display the user operations and results) and a StatusBar (to show messages to users). The GUI connects all the functions defined in each module, and allows users to apply the methodological process in a controlled manner. Figure 2.4 shows the operations integrated in pyColourimetry (top ToolBar), and its relationship with the steps defined in the project actions pipeline (bottom pipeline).

The project workflow phases are as follows:

- Project: An active project is required to start the process. It is possible to open a project or create a new one. Each project has a log file associated, in which all the operations carried out are recorded. This file can be checked by users anytime (Figure 2.5).

- Data acquisition: For characterization procedure, two different data sets are required: RGB from an image, and the CIE XYZ from a color checker. pyColourimetry allows users to collect direct colorimetric data or import previous measurements as CSV files; load spectral 
data from TXT files and convert to CIE XYZ; and to extract RGB data from images collected by different camera sensors (preferred in RAW format).

- Characterization: To compute the RGB-CIE XYZ transformation equations, and give the statistics for the model assessment.

- Sample: To apply the transform coefficients to full images, or to obtain the CIE XYZ values for specific specimens from an image.

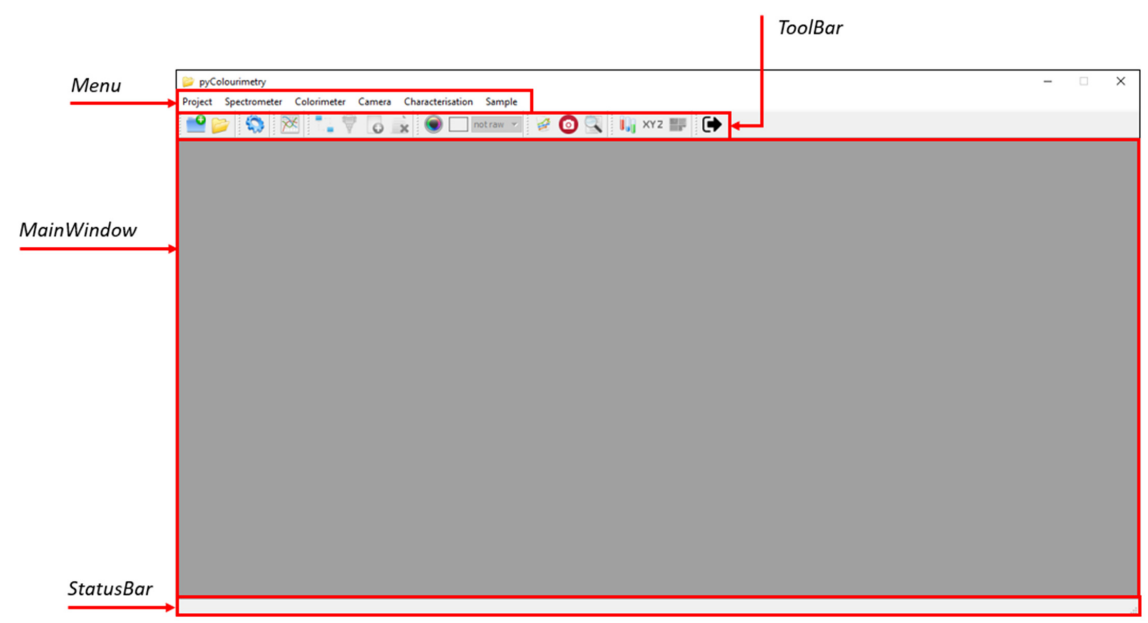

Figure 2.3: GUI

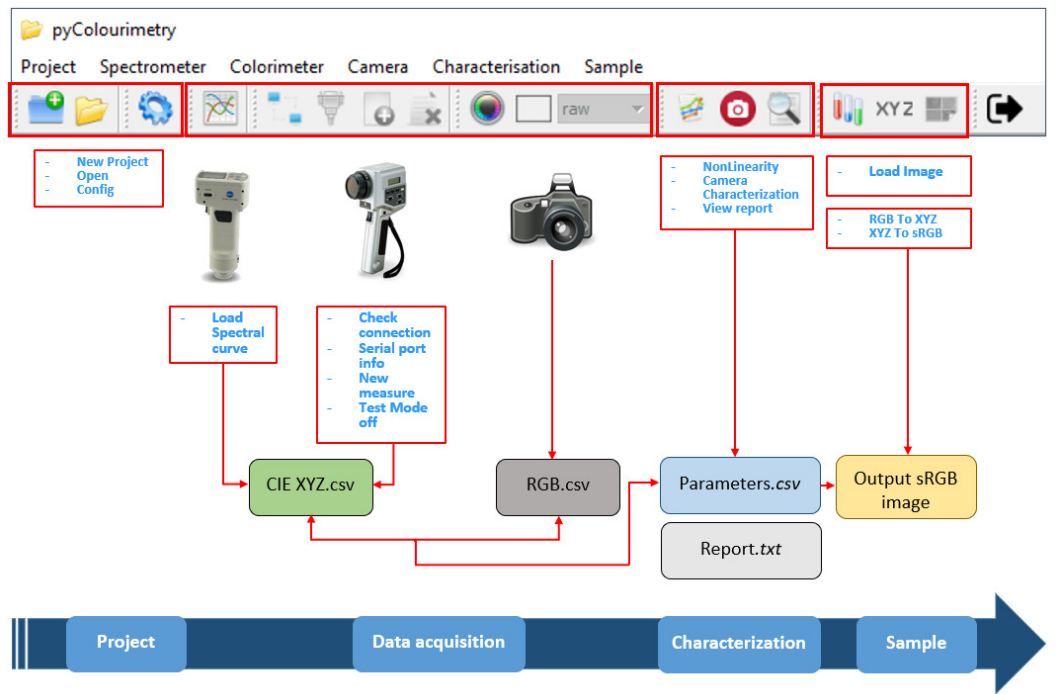

Figure 2.4: ToolBar options and their relationship with the project work pipeline 


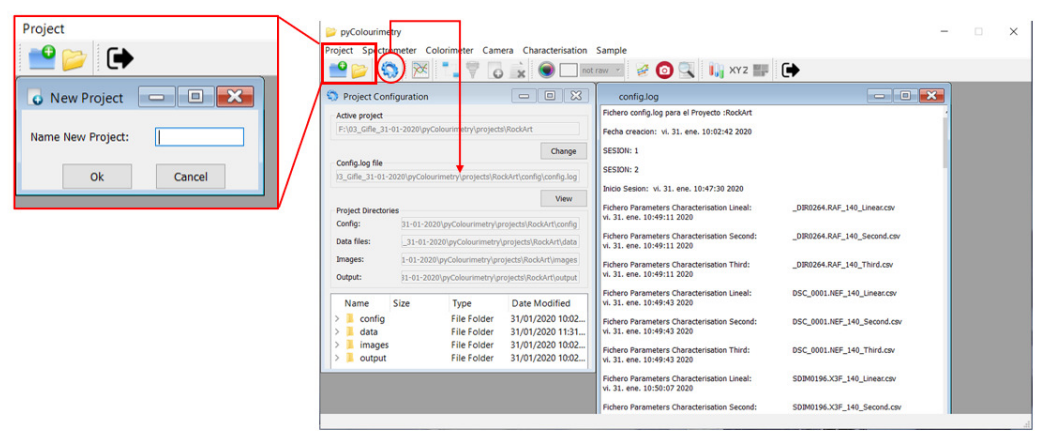

Figure 2.5: Project options

In the following subsections, we provide further details as pyColourimetry covers each of the project workflow phases enumerated above. However, we want to note that each of these steps is supported by a complex set of functions designed in the script modules, which serve as pyColourimetry body, while the GUI acts as brain interconnecting all the functions developed. Thus, the users are well informed during the project workflow, and can adjust the operations taking into account the specific requirements of each project.

\subsubsection{Serial port communication}

An outstanding pyColourimetry feature is that it allows users to take direct measurements using a Konica Minolta CS-100A colorimeter through the serialPort.py module. It is possible to connect the colorimeter according to the communication specifications of the manufacturer to retrieve colorimetric data (Figure 2.6). Additionally, it would be possible to add other instruments to pyColourimetry if the communication parameters are known.

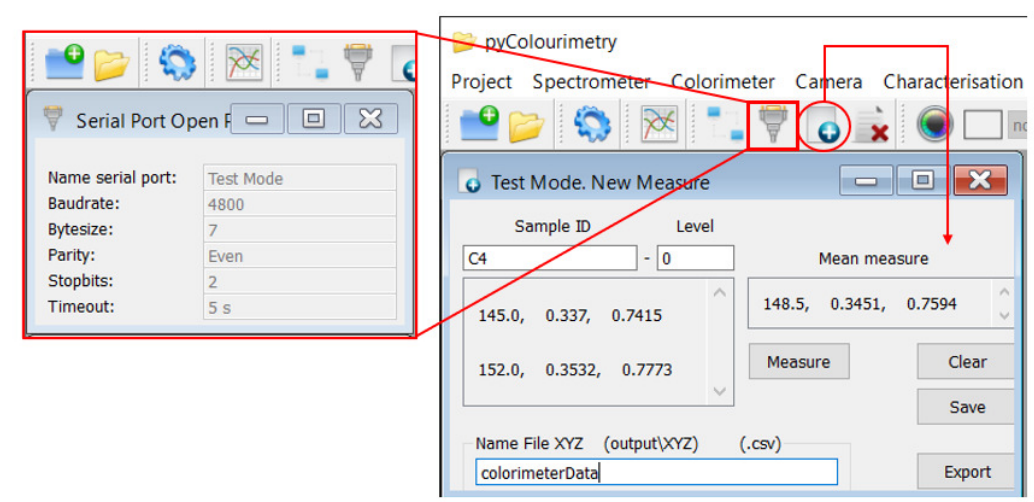

Figure 2.6: Colorimeter serial port connection

Figure 2.7 displays the workflow scheme to acquire direct measurement using the CS-100A colorimeter connected through pyColourimetry. The user should first check if the CS-100A 
colorimeter is connected to one of the computer ports. If the connection has been made correctly, the software returns the name of the port and the connection configuration, and the rest of the options in the menu and the toolbar is activated. However, a test mode was designed in the event that not active port is detected. The test mode allows simulating the connection with a virtual colorimeter, taken fictitious measurements. This mode was designed mainly as a debugging tool to check the correct performance of the code.

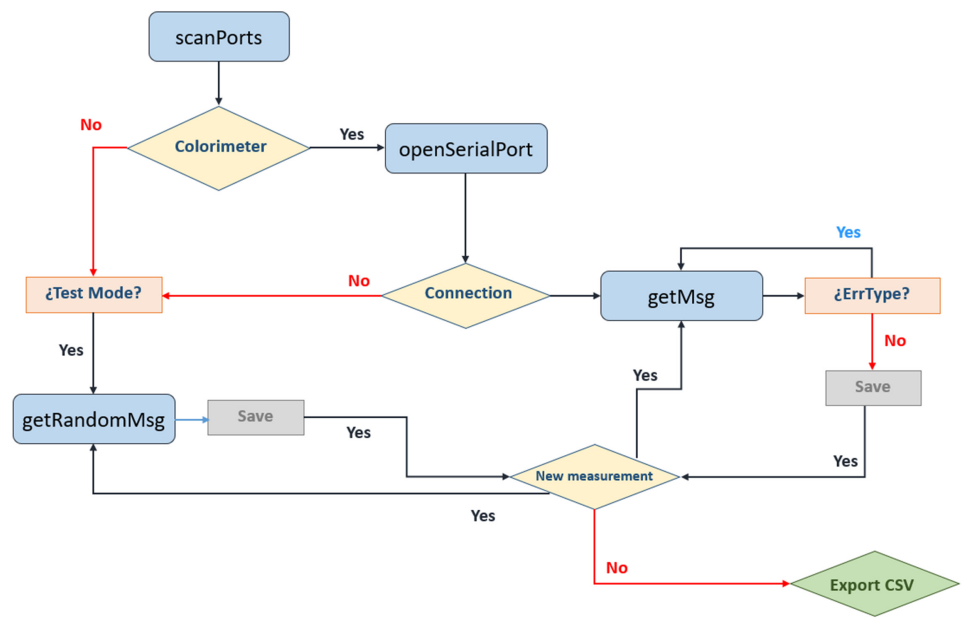

Figure 2.7: SerialPort module workflow scheme

As outlined in Figure 2.7, the main functions implemented in the serial port module are:

- scanPorts: Search for the colorimeter serial port connected.

- openSerialPort: Start communication with the colorimeter. If any port is detected, the Test Mode is available for users.

- getMsg: Message with the coordinates given by the colorimeter.

- getRandomMsg: Random measurement in Test Mode.

- ErrType: Error message to the user if communication with the colorimeter fails.

As a result of the direct measurement, the colorimetric data given by the colorimeter can be easily saved to a CSV file and loaded by the user when required. However, users can load measurement from different colorimetric instruments in properly formatted text files.

\subsubsection{Colorimetric data treatment}

Although there are specific Python libraries for colorimetric data processing (colormath, Table 2.3), an object-oriented module called myColour.py has been implemented in pyColourimetry, based on the main technical recommendations published by the CIE (Table in Figure 2.2, 
CIE (2018)). The myColour.py script allows users to handle the main CIE color spaces for the $2^{\circ}$ standard observer and the D65 illuminant such as CIE XYZ, Yxy, RGB, CIELAB and CIELCH, as well as to compute transformation among color spaces and calculate color differences between samples. In addition, the most widely CIE chromatic adaptation transforms (CATs) are included in our module (CIE, 2004), and two new derived from the von Kries model by numerical optimization (Bianco et al., 2010)

These are some of the operations programmed in our colorimetric module:

- CIE Color spaces: Yxy, XYZ, CIELAB, CIELCH

- Additional color spaces: RGB, LMS

- Functions for color space transform: YxyToXYZ, XYZToYxy, XYZToRGB, RGBToXYZ, XYZToCIELAB, CIELABToXYZ, CIELABToCIELCH, XYZToLMS,

- Color Difference equation: $\Delta E_{a b}^{*}$ (CIE (2018), Eq. 3.3)

- Chromatic Adaptation Transforms (CATs): von Kries, Bradford, Sharp, CMCCAT2000, CAT02, BS and BSPC.

The use of our colorimetric software offers several advantages. The functions implemented are designed to make calculations more flexible, and adapted to specific requirements. For example, in pyColourimetry, the user can specify the white reference used for the transformation among CIE XYZ and CIELAB color spaces. On the contrary, this option is not viable in the color transformations functions available in the colormath package; only the use of theoretical white references are allowed. However, we based the spectral data treatment on the color conversion function implemented from colormath, since it is a complex operation. Thus, it is possible to load spectral datasets collected with spectrometers from TXT files and convert to CIE XYZ data and export them to a CSV file (Figure 2.8).

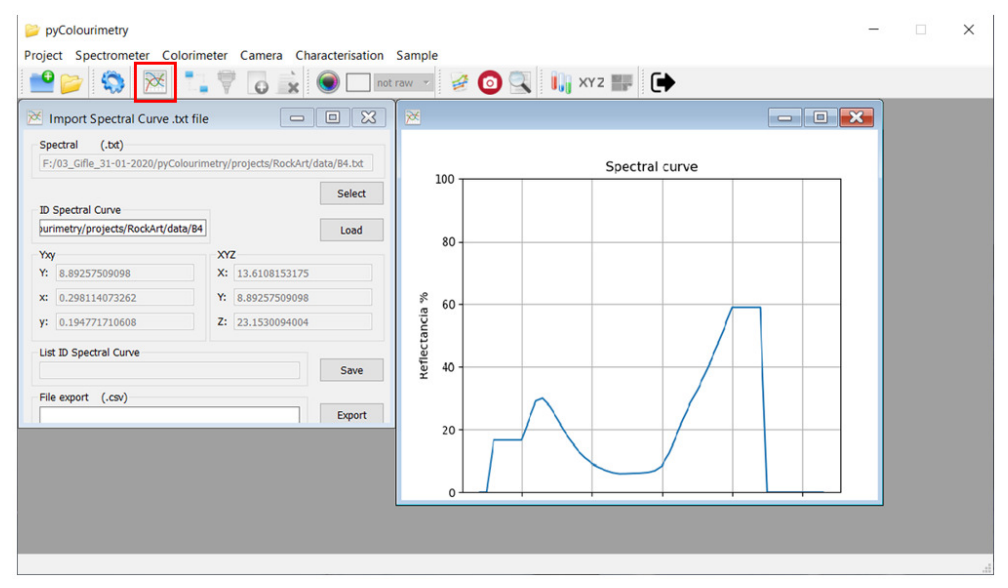

Figure 2.8: Spectral curve generation example 


\subsection{Camera characterization procedure}

Although in Chapter 3 we provide further explanation about image-based camera characterization procedure, it is required a brief description of the framework proposed. Figure 2.9 displays the basic pipeline for camera characterization, which establishes the following steps: (1) Data acquisition; (2) Training data; (3) Model fit; (4) Model assessment; and (5) Output sRGB image.

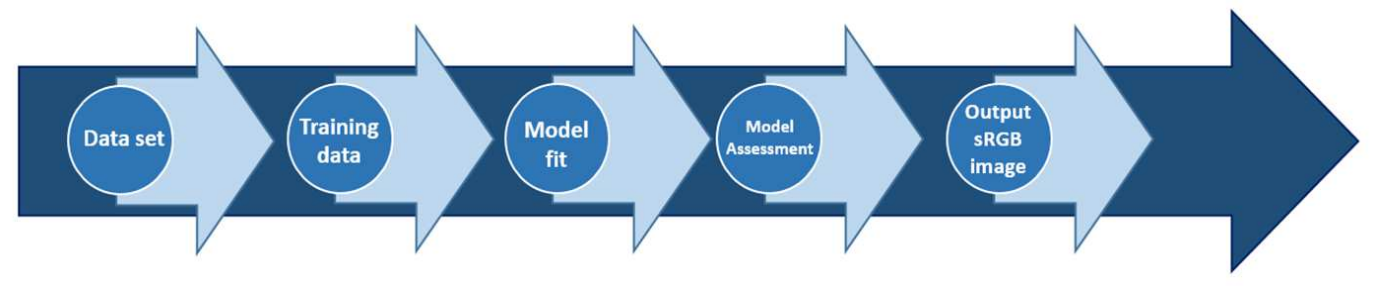

Figure 2.9: Basic characterization workflow.

The workflow described above is fully integrated into pyColourimetry software (cf. Figure 2.9 and Figure 2.10). The graphic scheme shown in Figure 2.10 was designed based on a set of GUI screen captures from pyColourimetry. Thus, it can be clearly observed how users can apply each one of the steps exposed using pyColourimetry. Hereunder we give additional comments on these basic steps.

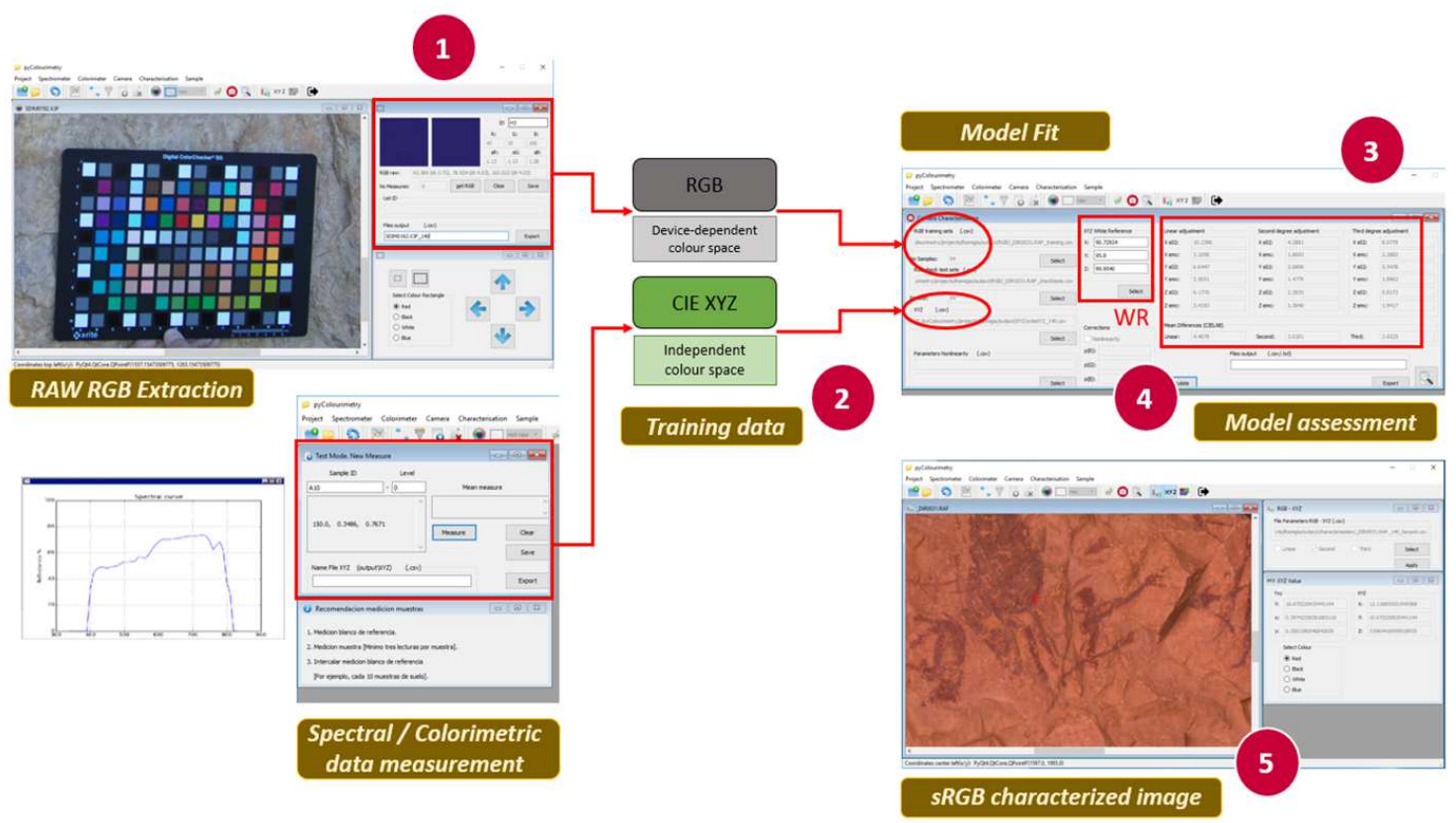

Figure 2.10: Camera characterization procedure using pyColourimetry software 
In camera characterization, we need several sets of samples with RGB data from the selected color patches found in the original image. Interestingly, pyColourimetry is capable of extracting RGB data in RAW format from the main commercial digital image formats (such as RAF, NEF, $\mathrm{X} 3 \mathrm{~F}$, CR2). The theoretical CIE XYZ tristimulus values from the color checker are required as well. Both data sets were used as training data for setting the characterization parameters which establish the relationship between the RGB data and the CIE XYZ coordinates. Additionally, a testing sample to evaluate the quality of the applied adjustments is required.

As indicated above, the CIE XYZ data can be measured directly using the CS-100A colorimeter and saved as a CSV file. Nevertheless, the colorimetric data can be taken using a different instrument, importing the data through exchange files in a specific format.

By means of the camera characterization, we establish the relationship between the device-dependent RGB data and the tristimulus values defined by the CIE standard colorimetric observer. The colorimetric camera characterization process is carried out in two steps. First, non-linearity correction (linearity.py) of the pixel values is computed when is required (for RAW RGB data it is not mandatory since they are linear). In the second step, the coefficients of the RGB to CIE XYZ transformation equations are obtained from a number of selected patches of the color chart (characterization.py).

In pyColourimetry we implemented the polynomial method because it gives substantially the same results as other methods and offers lower pre-processing and computational cost. Three polynomial models were considered: linear, second and third-order. The number of coefficients to be determined is 3,10 and 19, respectively, depending on the degree of the polynomial. However, the use of polynomial regression models is a starting point. Chapter 4 provides a new model regression based on Gaussian Processes to improve the characterization results.

In order to evaluate the results obtained after characterization, pyColourimetry provides statistical estimators, the root-mean-square errors (RMSE) and residuals to analyze the quality of the applied adjustments. Additionally, color accuracy is measured by computing the $\Delta E_{a b}^{*}$ (see Eq. 3.3) difference formula (CIE, 2018), between the imaged results of the standard target patches and their theoretical color values. It also provides a report in TXT format when the regression is finished. The generated document allows users to select the optimum number of coefficients for device characterization as well.

Once the parameters are calculated, we apply the RGB to CIE XYZ transformation equations on the input image. However, a final transformation from CIE XYZ to sRGB space is still necessary to obtain the output image in a device-independent, physically-based color space, which can be rendered in all devices compatible with the sRGB color space. After computing all transformation parameters a conventional digital camera can be used to determine rigorous object colors simulating a colorimeter. 


\subsection{Conclusions}

The digital camera characterization procedure proves to be a suitable approach for correct color data measurement in cultural heritage documentation applications. Its use is widely justified since it is a low-cost, non-invasive technique, and a fast method to record accurate data. However, specific colorimetric software is required to carry out the camera characterization. The pyColourimetry software reported in this chapter can be considered as rigorous colorimetric software since it was developed and tested according to well-accepted recommendations published by the CIE. In addition, pyColourimetry covers every single step required for proper camera characterization, and gives the user full control of the overall computation process. It also facilitates data acquisition and measurement tasks as well as the processing of colorimetric samples, setting the polynomial parameters, and applying them to finally obtain the output image in the sRGB space. Another key aspect of pyColourimetry is the rigorous processing of RAW data from images regardless of the sensor being characterized.

In conclusion, the camera characterization procedure integrated into pyColourimetry allows the correct color specification in a non-subjective way, following a low-cost solution for color communication and dissemination. Once the camera is characterized, users have the potential to obtain output images in the sRGB space that is independent of the sensor of the camera. Besides, we are working to adapt pyColourimetry functionalities to future specific needs of both information users and information providers.

In the following chapter, we present the results achieved on a rock art study which confirm that the framework proposed for camera characterization through pyColourimetry is a suitable methodology for proper color specification in cultural heritage color documentation. 


\section{3}

\section{Image-based camera characterization}

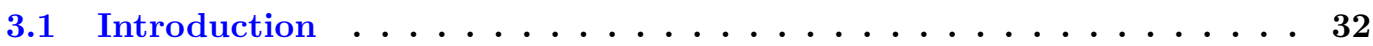

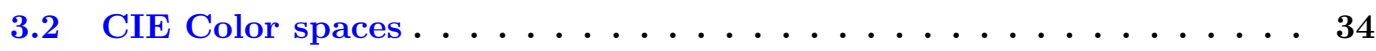

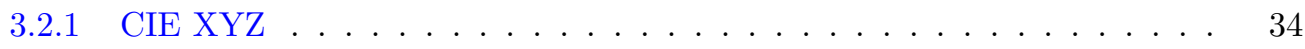

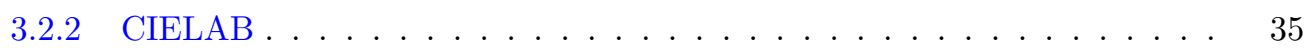

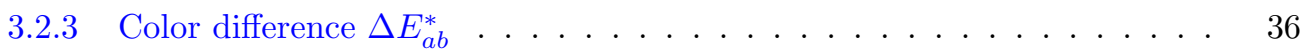

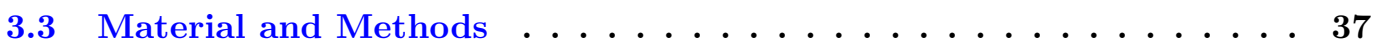

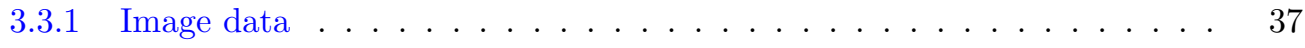

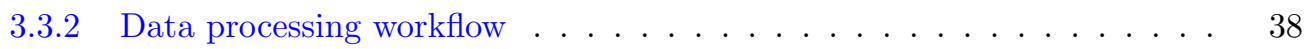

3.3.3 First stage: Characterization of the digital camera . . . . . . . . . 39

3.3.4 Second stage: Transformation to sRGB space . . . . . . . . . . . . . 40

3.3.5 RAW data processing . . . . . . . . . . . . . . . . . . 41

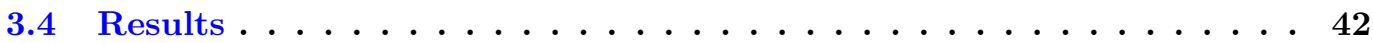

3.4 .1 TIFF data results . . . . . . . . . . . . . . . 43

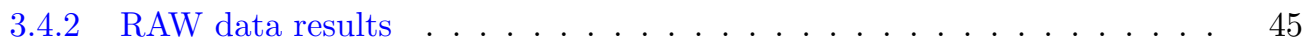

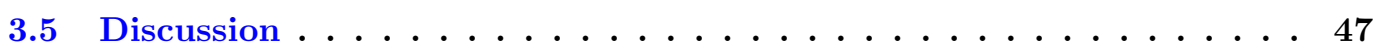

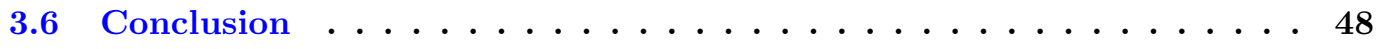




\subsection{Introduction}

Color is a fundamental feature for proper cultural heritage documentation in general, and archaeological rock art documentation in particular. The correct determination of color provides vital information, not only at a descriptive level, but also, especially, at a technical and quantitative level. This allows a better understanding of specimens in the study area and provides useful information regarding the origin and aging of the pigments.

Traditionally, rock art documentation methods were restricted to subjective procedures based on direct observations of the researcher with the use of color charts (for instance the Munsell Color Book). Despite its clear advantages, this methodology entails practical and technical limitations, affecting the results obtained in determining the color (Ruiz and Pereira, 2014). The problem is that color is a matter of perception and subjective interpretation. There is no single physical and universal scale for its measurement (Brill, 1996, Lang, 1997). In the particular case of visual assessments, even if several observers examine the same object, they can obtain different references and experiences and may express the same color stimulus with completely different words. It is obvious that verbal expressions cannot be used to communicate color information.

Moreover, in the last decades, cultural heritage documentation processes have largely benefited from the application of digital recording methods, imagery analysis software and technologies that offer great advantages over the traditional techniques (Iturbe et al., 2018). More and more it is becoming frequent to combine classical documentation techniques based on perceptual procedures with rigorous procedures, supported by colorimetric measurements and digital images.

The drawback of those digital recording methods stems from the digital cameras themselves which capture color information in the well-known RGB color space. Nevertheless, the device-dependent nature of RGB data makes them unsuitable for rigorous colorimetric purposes since they do not satisfy the Luther-Yves condition (Hong et al., 2001, Sharma, 2003), that is, the output RGB data do not directly correspond to the device-independent tristimulus values based on the CIE standard colorimetric observer (CIE, 2018). Thus, a colorimetric procedure is required in order to use the data provided by the digital camera sensor.

A common and acceptable approach is the digital camera characterization procedure. By characterization we refer to the determination of the transformation equations so that the acquired RGB information is brought into physically-based color spaces such as those introduced by the CIE, that is, the CIE XYZ space and its derivatives. In this way, a conventional digital camera could be used for rigorous color determination, somehow simulating a colorimeter (Balasubramanian, 2003, Martínez-Verdú et al., 2003, Westland et al., 2012). Once characterized, we can work in a physical color space, which is independent of the device and comparable with other devices already characterized.

The camera characterization framework proposed in this chapter is based on objective methods that are independent of the observer experience. It combines the direct method, based on colorimetric measurements obtained using specific instruments (colorimeter and spectrophotometer), and the indirect method, using digital images with RGB data (Figure 3.1). 


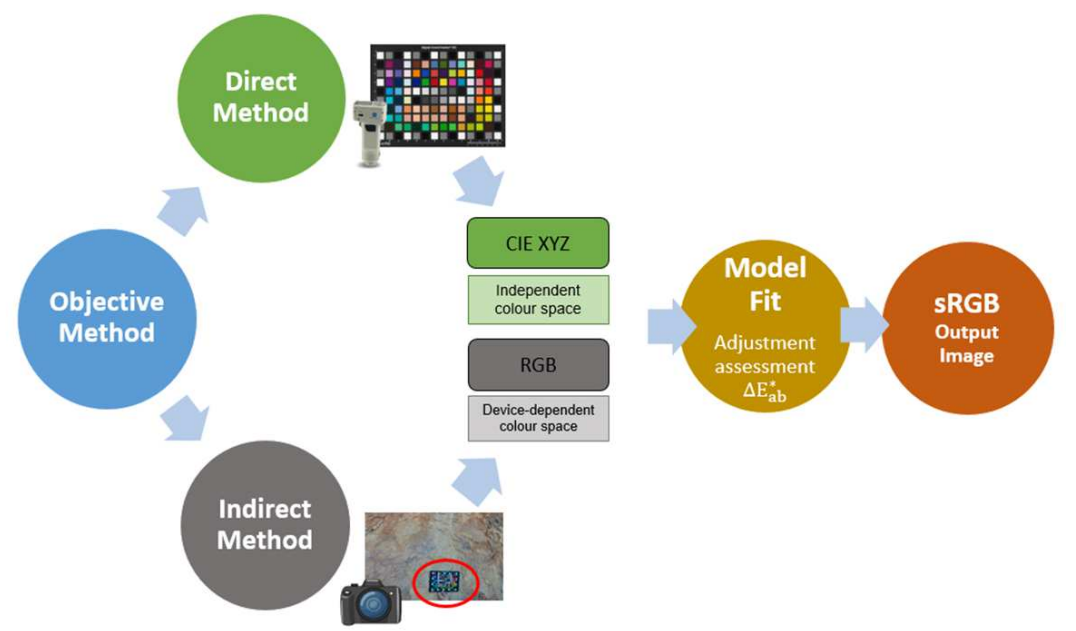

Figure 3.1: Image-based characterization workflow.

Different techniques are used for colorimetric camera characterization and numerous papers have been written regarding common models. Table 3.1 summarizes the main mathematical models used in camera characterization during the last decades. It seems clear that models based on polynomial transformation with least-squares regressions predominate, since they provide accurate results and are computationally simple (Hong et al., 2001, Westland et al., 2012).

Table 3.1: Mathematical models used for camera characterization procedure.

\begin{tabular}{llll}
\hline Author & Model & Samples & Color difference \\
\hline Vrhel and Trussell (1992) & Principal Component Analysis (PCA) & - & - \\
Hung (1993) & Look-up-table & 65 & $\Delta E_{u v}^{*}$ \\
Chang-Rak and Maeng-Sub (1999) & Multiple regression & $24,33,42,60,96$ & $C M C(1: 1)$ \\
Thomson and Westland (2001) & Parametric fitting & 24,120 & - \\
Pointer et al. (2001) & Polynomial Regression & 24,225 & $\Delta E_{a b}^{*}$ \\
Hong et al. (2001) & Polynomial regression & 264,2095 (textile) & $C M C(1: 1)$ \\
Cheung and Westland (2002) & Artificial Neural Networks (ANN) & 192 & - \\
Cheung et al. (2005) & Multispectral imaging technique & 166 & $\Delta E_{a b}^{*}$ \\
Bianco et al. (2007) & Pattern Search Optimization & 228 & $\Delta E_{a b}^{*}$ \\
Jackman et al. (2012) & Advanced Regression & 24 & $\Delta E_{a b}^{*}$ \\
Finlayson et al. (2015) & Root-Polynomial Regression & 96,180 & $\Delta E_{u v}^{*}$ \\
\hline
\end{tabular}

The colorimetric camera characterization based on polynomial regression models can be considered as an appropriate starting point. Currently, further studies have focused on spectral reflectance reconstruction (Liang and Wan, 2017, Heikkinen, 2018). However, results from previous research show that good results can be obtained using polynomial transformation equations, especially those of second or third-order (Martínez-Verdú et al., 2003, Westland et al., 2012). 
Cheung et al. (2004) made a comparative study between characterization by means of neural networks and polynomial regression. The study shows that although both approaches are capable of producing similar results when adequately used, polynomial models offer a better alternative for camera characterization. These are mathematically simpler models, require smaller training sets, and therefore less computing time.

Although the accuracy of the characterization generally increases as the number of terms in the polynomial grows (Bianco et al., 2009), in pyColourimetry software we implemented the polynomial method because it gives substantially the same results as other methods at lower pre-processing and computational cost (See Chapter 2). In particular, to obtain the RGB-CIE $\mathrm{XYZ}$ transformation equations, three polynomial models were considered in this study: linear, second and third-order.

Additionally, we should note that the camera characterization framework proposed is not a restrictive methodology, and can be used in a complementary way to other techniques for the study and conservation of rock art specimens, for instance, laser scanning together with photogrammetric techniques, which allow the generation of 3D photorealistic models of rock art paintings (Domingo et al., 2013).

In this chapter, we will present the results obtained in the characterization of a picture with Levantine rock art motifs using the pyColourimetry software. Prior to these results, we briefly describe the main CIE color spaces which are used in this study and other essential colorimetric elements to add some context to the reader.

\subsection{CIE Color spaces}

Since 1931, the CIE has developed systems to express color numerically. Color spaces define the quantitative relationship between physical signals in the electromagnetic visible spectrum, and physiological perceived colors in human vision. The mathematical relationships that define these color spaces are essential tools for color management (CIE, 2018).

Two well-known color spaces that provide consistent approaches in relation to the human visual system are the CIE xyY and the CIE $L^{*} a^{*} b^{*}$, both based on the so-called CIE XYZ tristimulus values.

\subsubsection{CIE XYZ}

The CIE 1931 XYZ color space allows any color stimulus (usually expressed in terms of radiance at fixed wavelength intervals in the visible spectrum) to be represented with three parameters XYZ called tristimulus values. CIE XYZ tristimulus values are fundamental measures of color and are directly used in a number of color management operations. This color space is actually a reference to define many other color spaces.

It is based on the additive color mixing principle. All color signals can be matched by the additive mixture of three primaries. In this color space, the primary colors used are not real colors, in the sense that they cannot be generated with any light spectrum. 
The second coordinate $\mathrm{Y}$ represents the luminance, which is the total radiation reflected in the visible spectrum. $\mathrm{Z}$ is quasi-equal to blue stimulation (or the $\mathrm{S}$ cone response of the human eye), and $\mathrm{X}$ is a linear combination of cone response curves chosen to be non-negative (Westland et al., 2012, CIE, 2018).

The CIE XYZ tristimulus values (Eq. 3.1) can be obtained as follows (CIE, 2018):

$$
\begin{aligned}
X & =k \sum_{\lambda} \phi_{\lambda}(\lambda) \tilde{x}(\lambda) \Delta \lambda \\
Y & =k \sum_{\lambda} \phi_{\lambda}(\lambda) \tilde{y}(\lambda) \Delta \lambda \\
Z & =k \sum_{\lambda} \phi_{\lambda}(\lambda) \tilde{z}(\lambda) \Delta \lambda \\
k & =\frac{100}{\sum_{\lambda} S(\lambda) \tilde{y}(\lambda) \Delta \lambda}
\end{aligned}
$$

where $\phi_{\lambda}(\lambda)$ denotes the spectral distribution of the color stimulus function; $\tilde{x}(\lambda), \tilde{y}(\lambda), \tilde{z}(\lambda)$ are color-matching functions of a standard colorimetric observer; $k$ is a normalizing constant; and $S(\lambda)$ is the relative spectral power distribution of the illuminant.

\subsubsection{CIELAB}

The three-dimensional color space produced by plotting CIE tristimulus values XYZ in rectangular coordinates is not visually uniform (CIE, 2018). Equal distances in this space do not represent equally perceptible differences between color stimuli. For this reason, in 1976, the CIE introduced two new color spaces (CIELAB and CIELUV) whose coordinates are non-linear functions of $\mathrm{X}, \mathrm{Y}$ and $\mathrm{Z}$. This non-linear transform of the XYZ values provided partial solutions to both the problems of color appearance and color difference.

The CIE $1976 L^{*} a^{*} b^{*}$ color space provides a three-dimensional color space where the $a^{*}-b^{*}$ axes form a plane to which the $L^{*}$ axis is orthogonal. It separates the color information into lightness $\left(L^{*}\right)$ and color information $\left(a^{*}, b^{*}\right)$ on two axes known as red/green $\left(a^{*}\right)$ and yellow/blue $\left(b^{*}\right)$. The lightness of a color stimulus ranges from 0 representing black to 100 representing white. As the position of color moves from the central region toward the edge of the sphere, its saturation (or chroma) increases.

The transformation from tristimulus values to $L^{*} a^{*} b^{*}$ coordinates is given by the well-known equation (CIE, 2018):

$$
\begin{aligned}
L^{*} & =116 \cdot f\left(\frac{Y}{Y_{n}}\right)-16 \\
a^{*} & =500 \cdot\left[f\left(\frac{X}{X_{n}}\right)-f\left(\frac{Y}{Y_{n}}\right)\right] \\
b^{*} & =200 \cdot\left[f\left(\frac{Y}{Y_{n}}\right)-f\left(\frac{Z}{Z_{n}}\right)\right]
\end{aligned}
$$


where

$$
\begin{aligned}
f\left(\frac{X}{X_{n}}\right) & = \begin{cases}\left(\frac{X}{X_{n}}\right)^{\frac{1}{3}}, & \left(\frac{X}{X_{n}}\right)<\left(\frac{6}{29}\right)^{3} \\
\left(\frac{841}{108}\right) \cdot\left(\frac{X}{X_{n}}\right)+\left(\frac{4}{29}\right), & \left(\frac{X}{X_{n}}\right) \leq\left(\frac{6}{29}\right)^{3}\end{cases} \\
f\left(\frac{Y}{Y_{n}}\right) & = \begin{cases}\left(\frac{Y}{Y_{n}}\right)^{\frac{1}{3}}, & \left(\frac{Y}{Y_{n}}\right)<\left(\frac{6}{29}\right)^{3} \\
\left(\frac{841}{108}\right) \cdot\left(\frac{Y}{Y_{n}}\right)+\left(\frac{4}{29}\right), & \left(\frac{Y}{Y_{n}}\right) \leq\left(\frac{6}{29}\right)^{3}\end{cases} \\
f\left(\frac{Z}{Z_{n}}\right) & = \begin{cases}\left(\frac{Z}{Z_{n}}\right)^{\frac{1}{3}}, & \left(\frac{Z}{Z_{n}}\right)<\left(\frac{6}{29}\right)^{3} \\
\left(\frac{841}{108}\right) \cdot\left(\frac{Z}{Z_{n}}\right)+\left(\frac{4}{29}\right), & \left(\frac{Z}{Z_{n}}\right) \leq\left(\frac{6}{29}\right)^{3}\end{cases}
\end{aligned}
$$

where $X, Y, Z$ are the tristimulus values of test color stimulus; and $X_{n}, Y_{n}, Z_{n}$ are the corresponding tristimulus values of a specified white color stimulus.

\subsubsection{Color difference $\Delta E_{a b}^{*}$}

The CIE XYZ color space does not match well to the human perception of color differences. The color differences perceived equally in different color regions can lead to very different distances in the CIE XYZ color space (Luo, 2006). In classical colorimetry, color difference metrics are determined using formulas based on the CIELAB color space that is more perceptually uniform than the CIE XYZ space. The non-linear transform of tristimulus values in the CIELAB equations allows the Euclidean distance between two points in the new space to better predict the visual color difference between the color stimuli represented by two points $(\Delta E)$.

Given a pair of color stimuli in CIELAB space, we establish the $\Delta E_{a b}^{*}$ or CIE76 color difference by the following equation (CIE, 2018):

$$
\Delta E_{a b}^{*}=\sqrt{\left(\Delta L^{*}\right)^{2}+\left(\Delta a^{*}\right)^{2}+\left(\Delta b^{*}\right)^{2}}
$$

It is advisable to work with CIELAB differences since we work in a uniform space, whereas this condition is not fulfilled in the CIE XYZ color space. It is for this reason that CIELAB color differences are useful to establish color tolerances between sample measurements and standards, and determine if the samples are considered acceptable. The criterion that allows us to establish color tolerances is based on the concept of "just noticeable difference" (JND), that is, a hardly perceptible difference between two sensory stimuli.

If $\Delta E_{a b}^{*}$ approximates to 2.3, being in any case less than to 3 (Mahy and Van Eycken, 1994, ISO, 2004) it is accepted that color differences are hardly perceptible by the human eye. Further, in cultural heritage digitalization, we can use two guidelines that provide measures to assess the color quality of digital images: FADGI (Federal Agencies Digitization Guidelines Initiative, (FADGI, 2016, Files and Griggs, 2016)) from the US and Metamorfoze (Netherlands national programme for the preservation of paper heritage (Metamorfoze, 2012, Van Dormolen, 2012)) from the Netherlands. The Metamorfoze guideline measures color quality using the CIE76 color 
difference formula, and defines a tolerance average ("color accuracy") $\Delta E_{a b}^{*}$ of 4 (Van Dormolen, 2012, Korytkowski and Olejnik-Krugly, 2017). In this study, we set the maximum acceptable value $\Delta E_{a b}^{*}$ to three CIELAB units.

There are a number of proposed improvements to the original $\Delta E$ calculation. Although CIEDE2000 is the most recent standard to compute color differences (CIE, 2014) we used the CIE76 because it fits better with typical working environments found in archaeological sites. Moreover, the CIEDE2000 report establishes a number of "reference conditions" such as sample size (4 degrees), sample-sensor separation (contact) and sample homogeneity (textureless) which are too demanding in practical archaeological applications. None of these conditions can be totally guaranteed in archaeological sites or even in rock art specimens under laboratory conditions. Therefore, in this study, we used the CIE76 color difference formula (CIE, 2018) for analyzing the results achieved after camera characterization.

\subsection{Material and Methods}

\subsubsection{Image data}

It has been stated above that trichromatic digital cameras capture color information in the well-known RGB format. The signal generated by the digital camera is device-dependent. By means of the characterization we establish the relationship between device-dependent RGB values and the tristimulus coordinates defined by the CIE standard colorimetric observer.

The steps required for the characterization of the camera are: (1) to collect an image of a color chart; (2) to extract the RGB data from the image; (3) to obtain the CIE XYZ coordinates of the patches present in the color chart; and (4) to compute the transformation from RGB to CIE space. Although it is recommended to use RAW image files rather than processed or compressed image files such as JPEG or ECW, this functionality is rarely found in available packages.

The data used herein consist of a number of digital images taken on a rock art site, called "Cova Remigia" (Figure 1.1). Its vividly and graphically narrative scenes make it a unique space in Levantine rock. A Fujifilm IS PRO camera (with a $60 \mathrm{~mm}$ spherical lens) was used to take RAW images. This device has high sensitivity and a low noise CCD sensor with a depth of 14 bits/pixel (Table 1.1). The X-rite ColorChecker SG Digital Color Chart with 140 patches was included in the visual field of this rock art scene (Figure 3.2).

The CIE XYZ tristimulus coordinates of the color patches are usually provided by the manufacturer. However, it is recommended to perform a direct measurement that allows us to control the conditions of the data collection, which in general differ from those of the published chart. The instrument used in our experiments was the spectrophotometer Konica Minolta CM-600d, which was calibrated before the measurement sessions (Figure 2.1 c). An average of four measurements was obtained for each color patch using a standard $2^{\circ}$ observer and D65 illuminant. The data with the CIE XYZ coordinates were stored in a text file for further processing. 


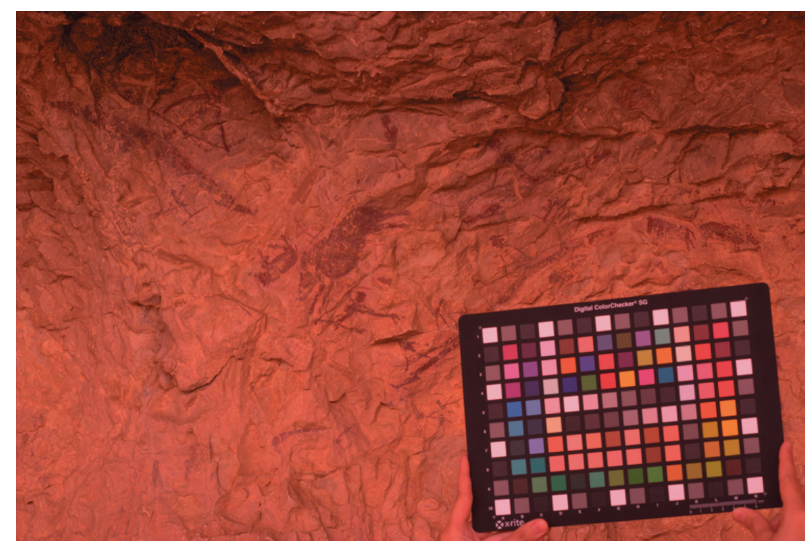

Figure 3.2: Example of data acquisition on site. Partial view of the Shelter V, Cova Remigia.

Finally, we obtained three subsets of samples (grey samples, training samples, and testing samples) with RGB data from a number of selected patches using the pyColourimetry software (Figure 3.3). We used these RGB data sets to determine the transformation equations, as well as to evaluate the results obtained after the characterization process.

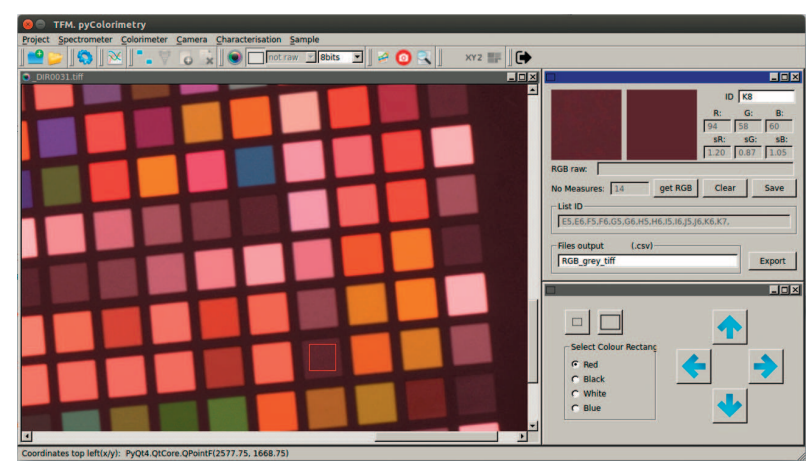

Figure 3.3: Measuring RGB data with pyColourimetry software.

\subsubsection{Data processing workflow}

For the characterization of the digital camera, we transformed the original data in the device-dependent RGB space into the physically-based CIE XYZ color space. Once the transformation equations are determined, we can represent the image in the sRGB color space.

In the methodological process, we considered the basic colorimetric recommendations published in the CIE report (CIE, 2018). Some fundamental aspects described in the report are the usage of illuminants, observers, reference standards for reflectance, viewing conditions, lighting, calculation of tristimulus values, chromaticity coordinates, color spaces, color differences and auxiliary formulas. 
Once the initial datasets are prepared, we differentiate two stages in the procedure. The first stage aims specifically at the characterization of the camera, which gives the coefficients of the polynomial transformation equations between the RGB and CIE XYZ spaces. In the second stage, the transformation equations are applied to the input image to obtain the definitive sRGB image (Figure 3.4).

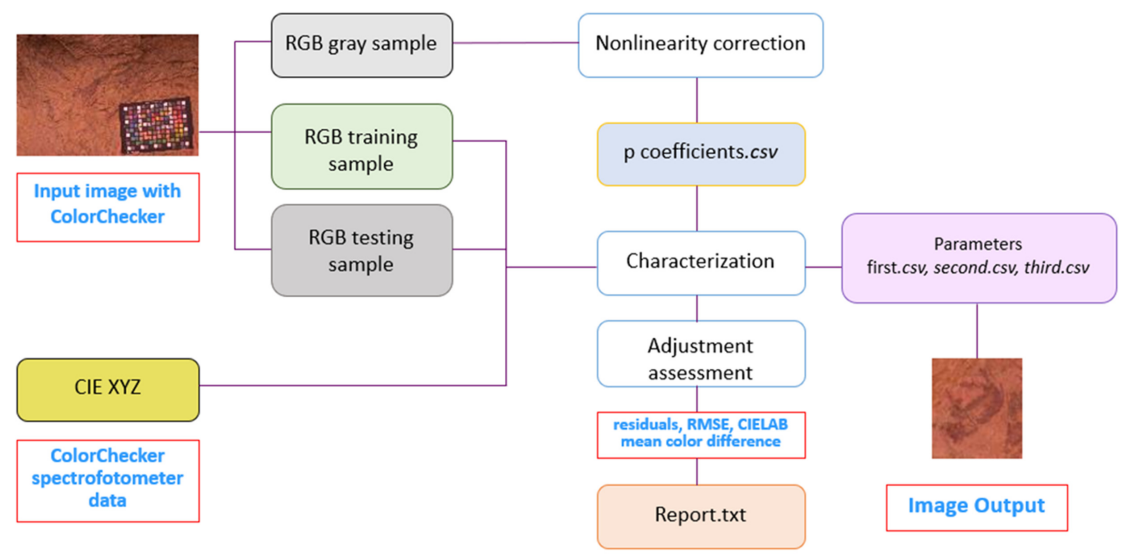

Figure 3.4: Flow chart to show the characterization implemented for digital cameras.

In the process, we need three sets of samples with RGB data from the selected color patches: (1) a grey sample to obtain the coefficients for the non-linearity correction; (2) a training sample to determine the transformation equations; and (3) a testing sample, which will allow us to evaluate the quality of the applied adjustments (Westland et al., 2012).

The first dataset contains the 15 grey-scale patches. The $p$ coefficients (Eq. 3.4) for the non-linearity correction are calculated from this first sample (Westland et al., 2012). After checking for linearity, the training sample data set is used to determine the characterization equations. A total of 54 patches of the most characteristic colors of the scene are selected to set the system of equations. The training dataset provides sufficient redundancies for the least-squares adjustment.

Finally, the equations are applied to the third testing sample dataset. The software provides statistical estimators and residuals to carry out the quality assessment of the applied adjustments and select the optimum coefficients for the characterization. This information is essential to make right decisions during the camera characterization process.

\subsubsection{First stage: Characterization of the digital camera}

The characterization is carried out in two steps. First, the need for non-linearity correction of the pixel values is analyzed. Although the initial response of the CCD sensor is almost linearly related to the intensity of the incident light, it is unlikely that the RGB output of the camera is linearly related to the CIE XYZ tristimulus values of the scene surface. The RAW RGB data are 
transformed by means of a complex sequence of operations to obtain RGB output values, such as pre-processing, linearization, white balance adjustment, demosaicing and color transformation (Ramanath et al., 2005).

It is recommended correction for the non-linearity of the pixel responses as a pre-processing stage in the characterization of cameras according to Eq. 3.4. The exponential $p$ coefficients are calculated from the relationship between the response of each RGB channel and the luminance (CIE Y tristimulus value), so that the relationship between the $C_{i}$ and $C_{i}^{\prime}$ values is linear (Westland et al., 2012). The formula is:

$$
C_{i}=C_{i}^{\prime p}
$$

where $C_{i}^{\prime}$ is the camera's response on channel $i$ (red, green and blue); $p$ is the exponential coefficient; and $C_{i}$ is the pixel value after the application of the correction for channel $i$. It is worth noting here that RAW values present highly linear behavior and this correction is not required.

In the second step, the coefficients of the RGB to CIE XYZ transformation equations are obtained from the selected patches of the color chart. The software implements three polynomial models: linear, second and third-order (Balasubramanian, 2003, Westland et al., 2012). The number of coefficients to be determined is 3,10 and 19 , respectively, depending on the degree of the polynomial (Table 3.2 ).

Table 3.2: Transformation coefficients matrix (first, second and third order).

\begin{tabular}{|c|c|c|c|c|c|c|c|c|c|c|c|c|c|c|c|c|c|c|c|}
\hline Order & & effi & ien & & & & & & & & & & & & & & & & \\
\hline 1 st & $\mathrm{R}$ & & B & & & & & & & & & & & & & & & & \\
\hline 2 nd & $\mathrm{R}$ & G & B & $R^{2}$ & $G^{2}$ & $B^{2}$ & RG & $\mathrm{RB}$ & GB & 1 & & & & & & & & & \\
\hline 3rd & $\mathrm{R}$ & G & B & $R^{2}$ & $G^{2}$ & $B^{2}$ & RG & $\mathrm{RB}$ & GB & $R^{3}$ & $G^{3}$ & $B^{3}$ & $R^{2} G$ & $R^{2} B$ & $G^{2} R$ & $G^{2} B$ & $B^{2} R$ & $B^{2} G$ & 1 \\
\hline
\end{tabular}

\subsubsection{Second stage: Transformation to sRGB space}

The coefficients from the previous stage allows the transformation of RGB pixel values into CIE $\mathrm{XYZ}$ tristimulus values. However, in order to adhere to current digital color standards, a final transformation from CIE XYZ to sRGB space is still necessary. This transformation is calculated following technical recommendations from the International Electrotechnical Commission (IEC) (IEC, 1998).

The matrix formulas to transform into the sRGB space are (Eq. 3.5):

$$
\left[\begin{array}{l}
R_{s R G B} \\
G_{s R G B} \\
B_{s R G B}
\end{array}\right]=\left[\begin{array}{ccc}
3,2406 & -1,5372 & -0,4986 \\
-0,9689 & 1,8758 & 0,0415 \\
0,0577 & -0,2040 & 1,0570
\end{array}\right] \cdot\left[\begin{array}{c}
X \\
Y \\
Z
\end{array}\right]
$$

where XYZ are the CIE tristimulus values. 
The CIE XYZ values are first transformed to non-linear $s R^{\prime} G^{\prime} B^{\prime}$ values as follows:

If $R_{s R G B}, G_{s R G B}, B_{s R G B} \leq 0.0031308$ then

$$
\begin{aligned}
& R_{s R G B}^{\prime}=12,92 \cdot R_{s R G B} \\
& G_{s R G B}^{\prime}=12,92 \cdot G_{s R G B} \\
& B_{s R G B}^{\prime}=12,92 \cdot B_{s R G B}
\end{aligned}
$$

otherwise, that is, if $R_{s R G B}, G_{s R G B}, B_{s R G B}>0.0031308$ then

$$
\begin{aligned}
& R_{s R G B}^{\prime}=1,055 \cdot R_{s R G B}{ }^{(1.0 / 2.4)}-0.055 \\
& G_{s R G B}^{\prime}=1,055 \cdot G_{s R G B}{ }^{(1.0 / 2.4)}-0.055 \\
& B_{s R G B}^{\prime}=1,055 \cdot B_{s R G B}{ }^{(1.0 / 2.4)}-0.055
\end{aligned}
$$

Then, the non-linear $s R^{\prime} G^{\prime} B^{\prime}$ values are converted to digital code values. This conversion scales the above $s R^{\prime} G^{\prime} B^{\prime}$ values by using the following equations (for a black count of 0 and white digital count of 255 for 8 -bits encoding):

$$
\begin{aligned}
& R_{8 b i t}=255 \cdot R_{s R G B}^{\prime} \\
& G_{8 b i t}=255 \cdot G_{s R G B}^{\prime} \\
& B_{8 b i t}=255 \cdot B_{s R G B}^{\prime}
\end{aligned}
$$

The outcome of the process is a digital image represented in a device-independent, physically-based color space, which can be rendered at maximum quality in devices compatible with the sRGB color space.

\subsubsection{RAW data processing}

An operating characteristic of modern digital cameras is that the captured RAW RGB data are transformed by means of a complex sequence of operations applied by the camera software, such as pre-processing, white balance adjustment, demosaicing and color transformation (Ramanath et al., 2005). All these operations alter the numerical values of the RGB pixel data used during the characterization, therefore affecting the calculation of the RGB - CIE XYZ transformation parameters. In our camera, the output of these operations is a tagged image file format (TIFF) picture, which minimizes the effects of the internal pre-processing.

A completely different approach for the camera characterization is to use the RAW RGB data instead of the processed TIFF data. This approach, dubbed as RAW-based characterization eliminates the influence of those automatic operations from the characterization process, and provides the advantage of computing the parameters of polynomial models straight from the sensor response (Figure 3.5). 


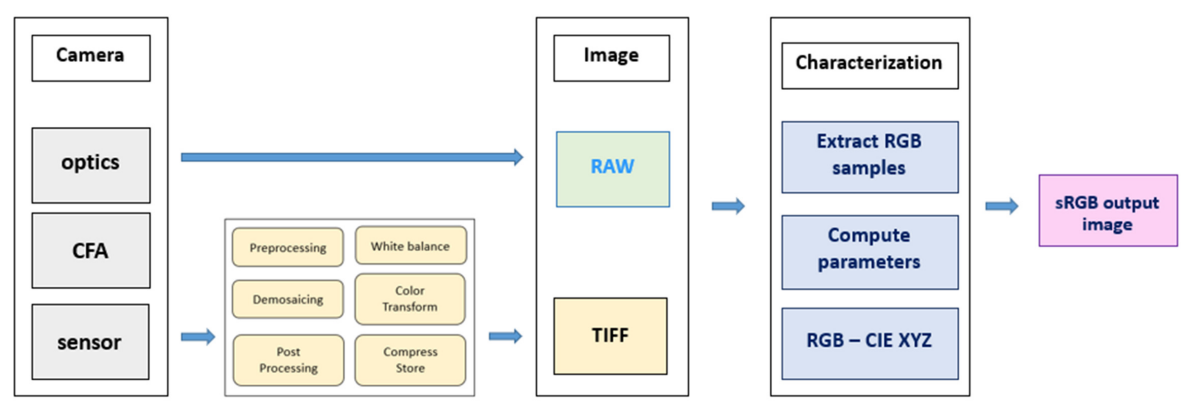

Figure 3.5: Comparative characterization workflow for RAW and TIFF images.

Although the use of RAW data is better for precise characterization (Westland et al., 2012), it is uncommon to find software packages with this characteristic. A number of technical complications may explain the lack of such systems, for instance, the handling of non-standard file formats or the computational load due to the RAW data high dynamic range. In spite of those complications, we decided to write a software package with RAW data processing characteristics called pyColourimetry (Chapter 2).

\subsection{Results}

From the sets of training and testing samples, three least-squares adjustments using first, second and third-order polynomials were conducted (Figure 3.6). In order to determine the optimal fit for the RGB to CIE XYZ transformation, we analyzed both the residuals and mean color differences. We also provide results for two different processing approaches based on TIFF images and RAW images.

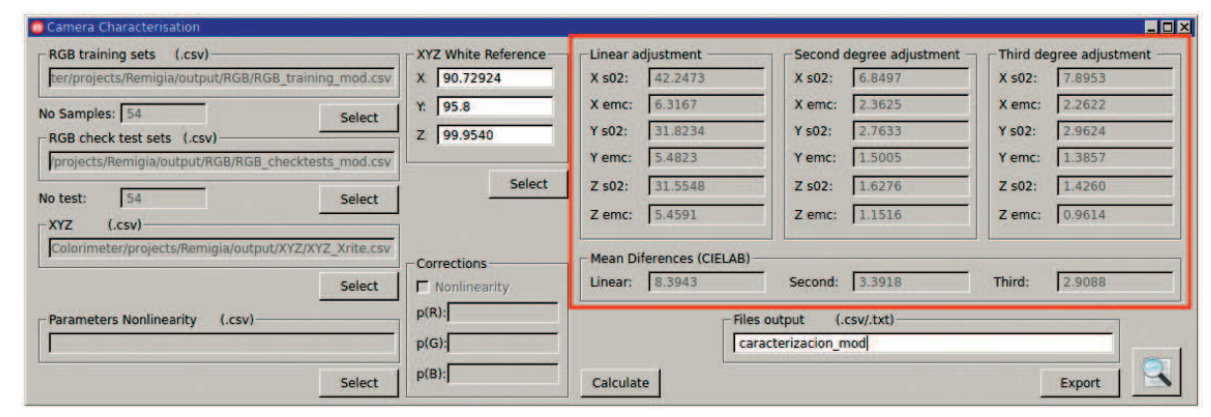

Figure 3.6: Adjustment results for the characterization of the digital camera (pyColourimetry). 


\subsubsection{TIFF data results}

Regarding tristimulus values residuals, there are no great differences between the transformations of second and third-order. In fact, these transformations gave the smaller residuals (Figure 3.7). As the degree of the polynomial increases, the statistical and residual results of the adjustment improve markedly. On the other hand, the adjustment with the greatest residuals is that of the linear transformation. Therefore, according to this initial criterion, the linear transformation should be discarded.

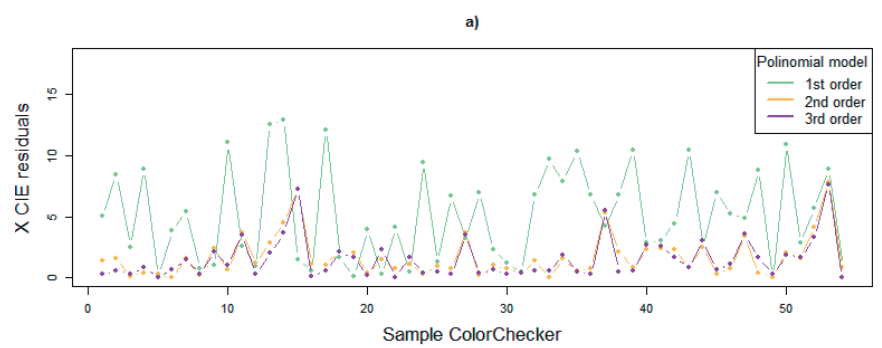

b)
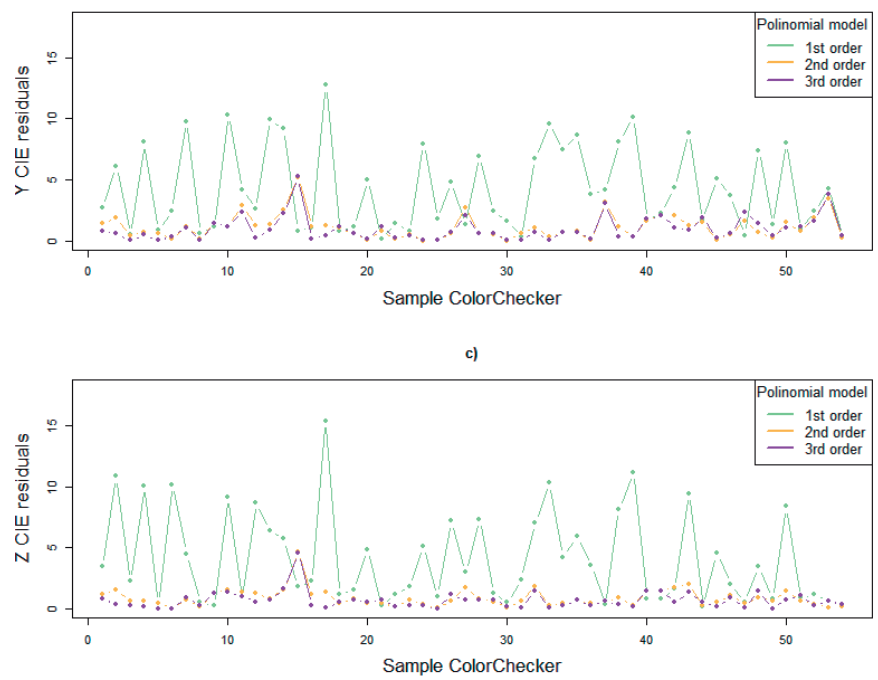

Figure 3.7: Residuals in the CIE XYZ space with TIFF data (1st, 2nd and 3rd order): a) X; b) $\mathrm{Y}$; c) Z.

The examination of the $\Delta E_{a b}^{*}$ CIELAB color differences shows again the contrast between the linear adjustment on the one hand, and the second and third-order adjustments on the other (Figure 3.8). In the linear transform, only a few color patches are below the acceptable value set at 3 CIELAB units. In accordance with this criterion, the linear polynomial fit is discarded. The second and third-order settings yield close values for most patches. It is therefore recommended, as other authors state in previous research, to use either the second or the third-order adjustments for the camera characterization (Balasubramanian, 2003, Westland et al., 2012). 


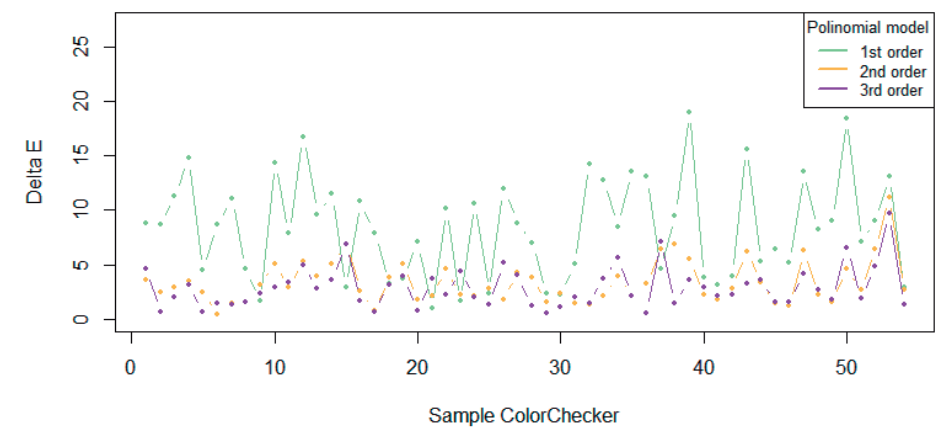

Figure 3.8: $\Delta E_{a b}^{*}$ CIELAB color differences with TIFF data.

As for the colorimetric characterization of the input image (Figure 3.9), the transformation equations computed with the three adjustments (first, second and third-order) were applied to three specimens that appear in the scene representing a wild boar, a hunter, and a nest (Sarriá Boscovich, 1988). As a result, we obtained output images in the sRGB space for the three transformations (Figures 3.10 and 3.13, upper row).

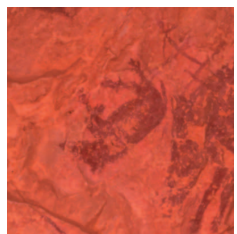

(a)

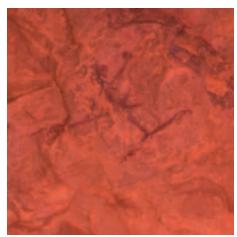

(b)

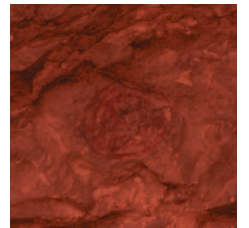

(c)

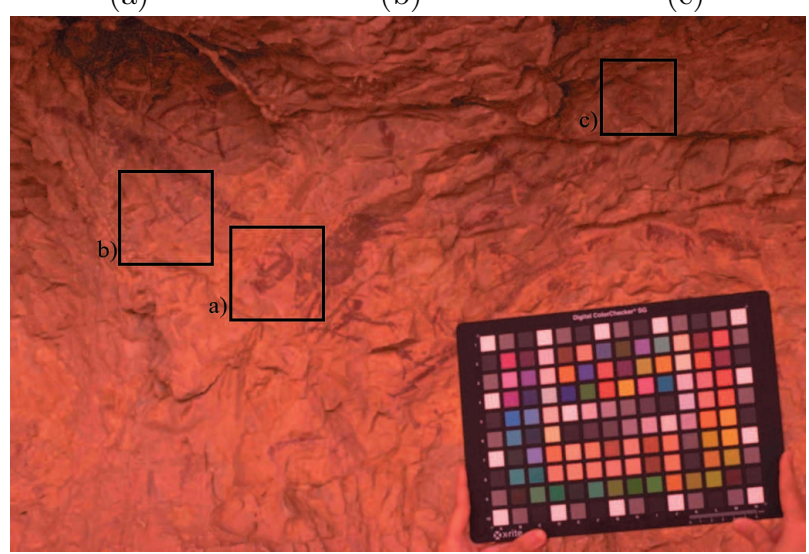

(d)

Figure 3.9: Specimens selected in the scene: a) wild board; b) hunter; c) nest; and d) layout of specimens. 


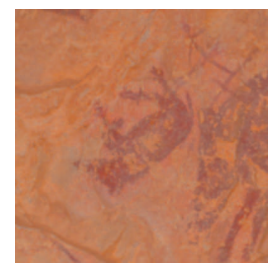

(a)

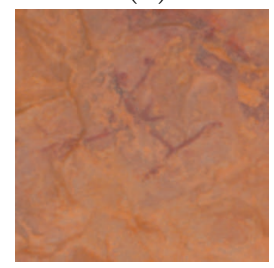

(d)

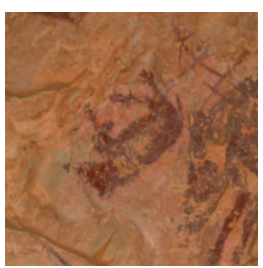

(b)

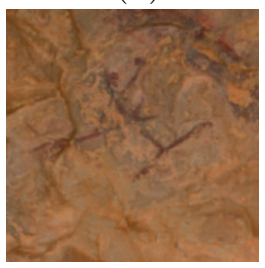

(e)

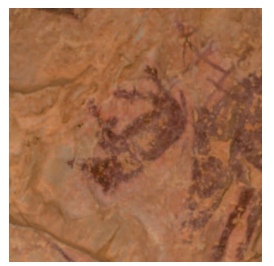

(c)

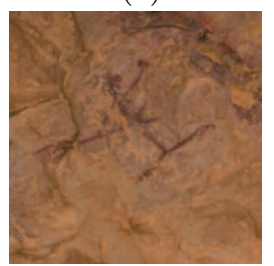

(f)

Figure 3.10: sRGB output images: a, b, c) Animal detail; d, e, f) Hunter detail; a, d) 1st order; b, e) 2nd order; c, f) 3rd order.

\subsubsection{RAW data results}

In this section, we give further numeric results and pictures obtained from the adjustments using TIFF data versus RAW RGB data (Table 3.3 and Figure 3.11). As in the previous section, the linear model adjusted with TIFF pictures gives large residuals, and therefore this model must be discarded for color processing. However, the linear model computed from the RAW data provides RMSE errors that are half the magnitude of those from the TIFF data.

Table 3.3: Comparative adjustment standard deviation results for the characterization of the digital camera with TIFF and RAW data.

\begin{tabular}{lllllll}
\hline & \multicolumn{3}{l}{ TIFF Picture } & \multicolumn{4}{c}{ RAW Picture } \\
\hline & Linear & Second & Third & Linear & Second & Third \\
$\mathrm{X}$ & 6.3167 & 2.3625 & 2.2622 & 3.1098 & 1.8692 & 2.2882 \\
$\mathrm{Y}$ & 5.4823 & 1.5005 & 1.3857 & 2.5051 & 1.4779 & 1.8963 \\
$\mathrm{Z}$ & 5.4591 & 1.1516 & 0.9614 & 2.4153 & 1.3640 & 1.9415 \\
& & & & & & \\
$\Delta E_{a b}^{*}$ & 8.3943 & 3.3918 & 2.9088 & 4.4079 & 3.0201 & 3.6570 \\
\hline
\end{tabular}

The second and third-order models have different behavior. In the TIFF data adjustments, the third-order model has the minimum residuals, whereas in the RAW adjustments, the second-order model yields the best result. A comparison between TIFF and RAW adjustments shows slightly better results in the TIFF adjustments. According to these figures, a user should apply either the third-order model with TIFF pictures or the second-order model with RAW pictures, with an apparent preference for the former. 
Color differences $\left(\Delta E_{a b}^{*}\right)$ follow a similar trend as that observed in XYZ residuals (Figure 3.12). The linear model gives the highest values, although in the RAW adjustment the difference is 4.4 CIELAB units, which is very close to the JND threshold. The color differences in the second and third-order models are reasonably acceptable. Again, a casual user might be tempted to use the third-order model with TIFF pictures since it has a lower value (2.9 units). However, this point deserves further discussion (see Section 3.5 and Figure 3.13).

Results from RAW data adjustments show that the polynomial of higher order does not improve the result (Table 3.3). Instead, the second-order polynomial gives the lower color difference with a similar value to that of the third-order polynomial with TIFF. The advantage of using the second-order model is the reduction of the order of the polynomial. In this line, authors recommend using polynomials of first or second-order, against polynomials of higher-order (Westland et al., 2012, Cheung et al., 2004).

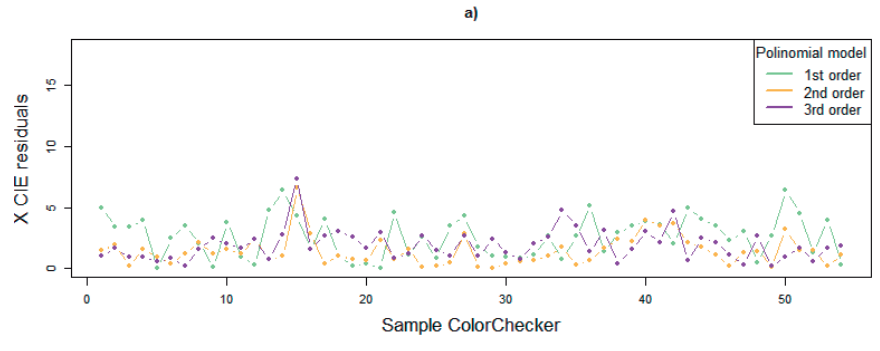

b)
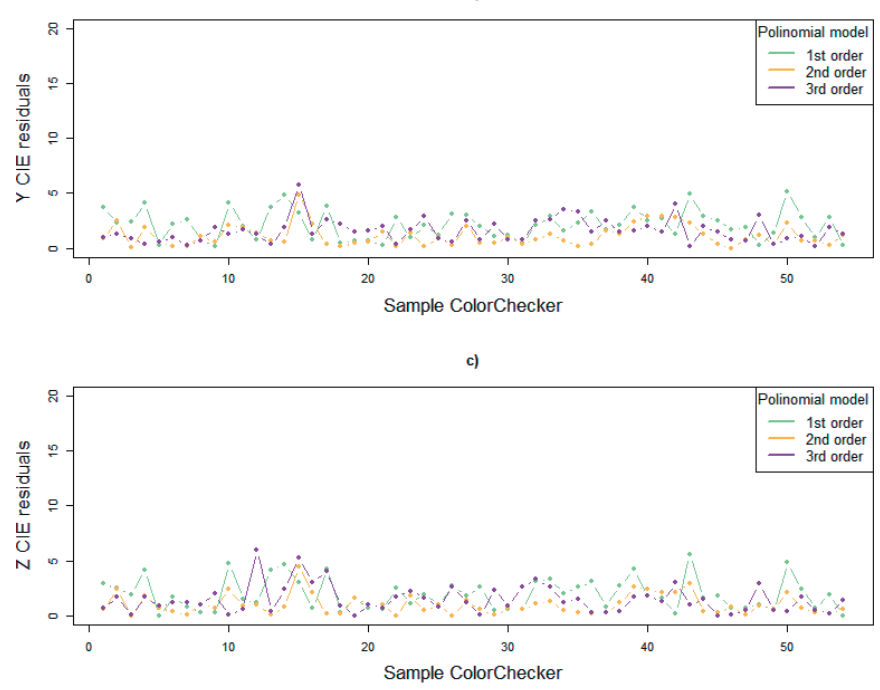

Figure 3.11: Residuals in the CIE XYZ space with RAW data (1st order; 2nd order; 3rd order): a) $\mathrm{X}$; b) $\mathrm{Y}$; c) Z. 


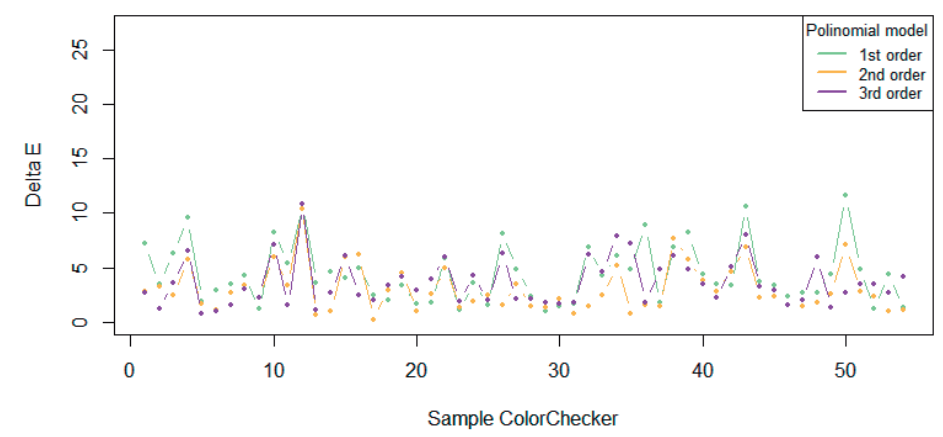

Figure 3.12: $\Delta E_{a b}^{*}$ CIELAB color differences with RAW data

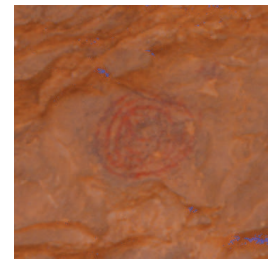

(a)

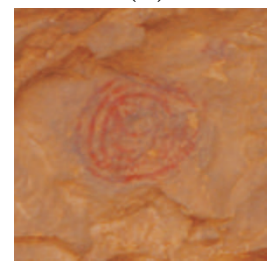

(d)

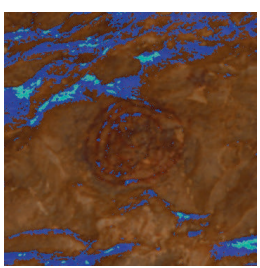

(b)

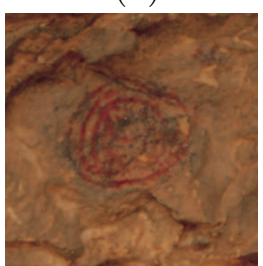

(e)

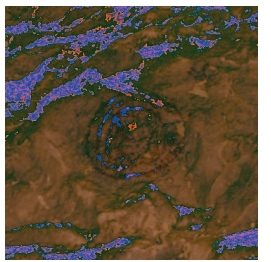

(c)

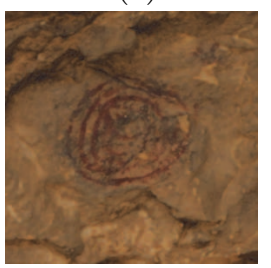

(f)

Figure 3.13: sRGB output images of the nest detail: a, b, c ) TIFF data; d, e, f ) RAW data; a, d) 1st order; b, e) 2nd order; c, f) 3rd order.

\subsection{Discussion}

The processing of TIFF files gives mean color differences $\Delta E_{a b}^{*}$ in CIELAB units of 8.4 for the linear model, 3.4 for the second-order model, and 2.9 for the third-order model. It is clear that color differences decrease numerically as we increase the degree of the polynomial. The worst result is obtained in the linear adjustment. In this case, the value of 3 CIELAB units established as tolerance in terms of JND is exceeded, so the linear model should be discarded since it does not adequately describe the relationship between the device RGB color space and CIE XYZ color space. The best results are obtained with the third-order polynomial, where the mean color difference is less than three CIELAB units. 
It is quite interesting to consider the results obtained in the third specimen, the nest. While the linear transformation might be thought to be discarded, after checking the output images obtained for both the wild boar and the hunter, in the latter specimen, it seems clear that the best results are obtained with the linear transformation. Despite the high residual value and mean $\Delta E_{a b}^{*}$ color differences, the final sRGB image obtained for the nest with the linear model is better (Figure $3.13 \mathrm{a}$ ). A smaller number of saturated pixels appears, and the color obtained after the characterization is closer to the color observed in the field site. In the images obtained with either the second or the third-order polynomial transformations, the number of saturated pixels is substantial. With this particular example, we proved that an increase in the order of the polynomial gave lower adjustment errors, but also created saturated sRGB output images that became useless for color documentation purposes.

It should be noted that the nest is in the upper-right area of the scene, which is less illuminated due to the prominent relief and curvature at the roof of the shelter. On the contrary, the central area of the image containing the other specimens is nearly vertical and flat. Obviously, the geometry of the cave affects lighting conditions, and this modifies fundamental colorimetric factors such as the reflectivity and color appearance of the object. In addition, the color chart was placed on the bottom-right area of the image with higher illumination.

The coefficients determined for the transformation equations with the TIFF data were therefore adequate for imaging areas with lighting conditions similar to those of the area where the color chart was placed, that is, the area close to the wild boar and hunter specimens. In dimly lit areas, as it is the case of the upper side of the image containing the nest specimen, these equations do not function properly.

The most interesting finding of this study was the high performance of the camera characterization when using RAW data. In general, the models created from RAW data gave the lower residuals in terms of both XYZ residuals and color differences. However, the true improvement of RAW-based characterization lays in the quality of the output images. The sRGB images created with the RAW-based characterization contain colors that remind one of the true colors at the archaeological site. Moreover, the process proved to be very robust, and worked well with specimens imaged under illumination conditions different to those of the color chart.

\subsection{Conclusion}

The results presented in this chapter confirm that the initial objective of establishing a methodological process for the characterization of digital cameras in archaeological research was achieved. It requires the joint processing of both radiometric and colorimetric data. The results are satisfactory and very promising for proper color documentation in rock art studies.

The proposed workflow for the characterization of digital cameras is adequate and takes the most important technical colorimetric aspects such as illuminants, standard observers, calculation of tristimulus values, chromaticity coordinates, color spaces, color differences and auxiliary formulas into account. 
According to the results of the study, we recommend applying the RAW-based characterization with second-order polynomial equations to transform data from the camera RAW RGB space to the CIE XYZ color space. The usage of a linear or third-order transformation should be restricted to very specific cases due to the higher residual values achieved in the adjustments.

One of such cases may be the processing of image features with illumination conditions different to those in the color chart area. Although these cases are rare and must be avoided in common practice, researchers must deal with them occasionally. Careful use of artificial light sources and detailed photographic shots containing the color chart next to the rock art specimen are good practices to avoid troublesome scenarios.

The computer application developed in this study, pyColourimetry, covers all steps of the methodological process, including the measurement and processing of colorimetric samples, the characterization and creation of the output image in the sRGB space. Moreover, our software gives the user full control of the overall computation process.

Future research must include the processing of RAW data from additional sensors and cameras with specific file formats, the optimization of the code implemented for the development of the pyColourimetry toolbox, as well as the inclusion of advanced statistical models to obtain improved transformation equations for the characterization of digital cameras. 



\section{4}

\section{A Gaussian Process Model for Camera Characterization}

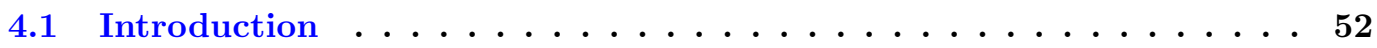

4.2 Materials and Methods . . . . . . . . . . . . . 53

4.2 .1 Case Study: Cova dels Cavalls . . . . . . . . . . . . . . . . . . 53

4.2.2 Image-Based Camera Characterization Methodology . . . . . . . . . . 53

4.2.3 Gaussian Processes for Camera Characterization . . . . . . . . . . . 55

4.2.3.1 Gaussian Process Model . . . . . . . . . . . . . . . 55

4.2.3.2 Bayesian Inference . . . . . . . . . . . . . . . . 56

4.2.4 Second-Order Polynomial Model _. . . . . . . . . . . . . . . 57

4.2.4.1 Bayesian Inference . . . . . . . . . . . . . . . . 58

4.2 .5 Model Checking and Comparison . . . . . . . . . . . . . . 59

4.2 .6 Induced Noise Analysis . . . . . . . . . . . . . . . . . . . . 59

4.3 Results and Discussion $\ldots \ldots \ldots \ldots \ldots$

4.3 .1 Model Performance Assessment . . . . . . . . . . . . . . . . . 60

4.3.1.1 CIE XYZ Residuals . . . . . . . . . . . . . . . 60

4.3.1.2 $\Delta E_{a b}^{*}$ Color Differences $\ldots \ldots \ldots \ldots \ldots$

4.3.1.3 Analysis of Color Chart Patches . . . . . . . . . . . . 64

4.3.1.4 Induced Noise Results . . . . . . . . . . . . . . . . 65

4.3 .2 Output sRGB Characterized Images . . . . . . . . . . . . . 68

$4.3 .3 \Delta E_{a b}^{*}$ Mapping Images . . . . . . . . . . . . . . . . 68

4.3.4 Rock Art Specimen Detail Images _ . . . . . . . . . . . . . 70

4.4 Conclusions . . . . . . . . . . . . . . . . . 72 


\subsection{Introduction}

The colorimetric characterization of digital cameras based on polynomial models is an appropriate starting point in this thesis; they are widely accepted, mathematically simpler and require smaller training sets and less computing time (Bianco et al., 2009, Cheung et al., 2004). Previous experiments using second-order polynomials applied in rock art paintings gave also good results (Molada-Tebar et al., 2018). However, they tend to be rigid models and suffer from overestimation or underestimation when many or few data are provided. Furthermore, the lack of reliable generalization of predictions in polynomial models is well-known, especially when extrapolating or in the case of modeling wiggly functions (Bishop, 2006). Therefore, it is desirable to improve the results by means of more flexible, robust and accurate models.

In this chapter, we introduce a novel approach for documenting rock art paintings based on a Gaussian process (GP) model. GPs are natural, flexible non-parametric models for $N$-dimensional functions, with multivariate predictors (input variables) in each dimension (Bernardo et al., 1998, Rasmussen and Williams, 2006). The defining property of a GP model is that any finite set of values is jointly distributed as a multivariate Gaussian function. A GP is completely defined by its mean and co-variance function. The co-variance function is the crucial ingredient in a Gaussian process as it encodes the correlation structure which characterizes the relationships between function values at different inputs. GP allows not only nonlinear effects and handling implicit interactions between covariates, but also improves generalization of function values for both interpolation and extrapolation purposes. Due to their generality and flexibility, GPs are of broad interest across machine learning and statistics (Neal, 1997, Rasmussen and Williams, 2006).

GP models are formulated and estimated within a Bayesian framework, and all inference is based on the multivariate posterior distribution. Computing the posterior distribution is often difficult, and for this reason, different computation approaches can be used. The Markov chain Monte Carlo (MCMC) is a sampling method that provides a sample of the joint posterior distribution of the parameters (Brooks et al., 2011, Durmus et al., 2018).

The GP model results were compared to the common approach based on polynomial regression models. The main advantage of non-parametric over parametric models is their flexibility (Green and Silverman, 1993, Ruppert et al., 2009). In a parametric framework, the shape of the functional relationship is a prespecified, either linear or nonlinear, function, limiting the flexibility of the modeling. In a non-parametric framework, the shape of the functional relationship is completely determined by the data, allowing for a higher degree of adaptability.

The goodness of fit and predictive performance of the models is assessed by analyzing the adjustment residuals and the leave-one-out cross-validation (LOOCV) residuals. The quality of the characterized image is also evaluated in terms of colorimetric accuracy by means of color differences among observed and fitted colors using the CIE framework. In addition, we evaluate the induced noise into the output image which is recognized as a drawback for some digital image processing applications such as image matching or pattern recognition. 


\subsection{Materials and Methods}

\subsubsection{Case Study: Cova dels Cavalls}

The working area is a rock art scene located in Shelter II of the "Cova dels Cavalls" (Figure 1.1). The images were taken using two different SLR digital cameras, a Sigma SD15 and a Fujifilm IS PRO. The images contain the hunting scene located in the central part of this emblematic archaeological site. Parameters such as focal, exposure time, aperture, and ISO were controlled during the photographic sessions for both cameras. Photographs were taken in the RAW format under homogeneous illumination conditions.

The main difference between the Fujifilm IS PRO and the Sigma SD15 cameras lies in the integrated sensors (Table 1.1). The Fujifilm incorporates a 12 megapixels Super CCD imaging sensor, with resolution of $4256 \times 2848$ pixels and a color filter array (CFA) with a Bayer pattern. The use of CFA implies that the color registered in every individual pixel is not acquired directly but as a result of interpolation between channels. On the other hand, the Sigma carries a three-layer CMOS Foveon $\mathbb{R}$ X3 sensor of $2640 \times 1760$ pixels, which makes it a true trichromatic digital camera, capable of registering color without interpolation (Sigma Corporation, 2012).

\subsubsection{Image-Based Camera Characterization Methodology}

The output RGB digital values registered by the camera depend on three main factors: the sensor architecture, the lighting conditions, and the object being imaged. Even assuming the same object and lighting conditions, other factors can still produce different RGB responses within and across scenes. Some elements such as the internal color filters or user settings (exposure time, aperture, white balance, and so on) can modify the output digital values. As a result, the original RGB data registered by the sensor cannot be used rigorously for the quantitative determination of color, and native RGB camera color spaces are said to be device-dependent. A way to transform the signal captured by the camera sensor into a physically-based, device-independent color space is by means of camera characterization (See workflow in Figure 4.1 and Chapter 1).

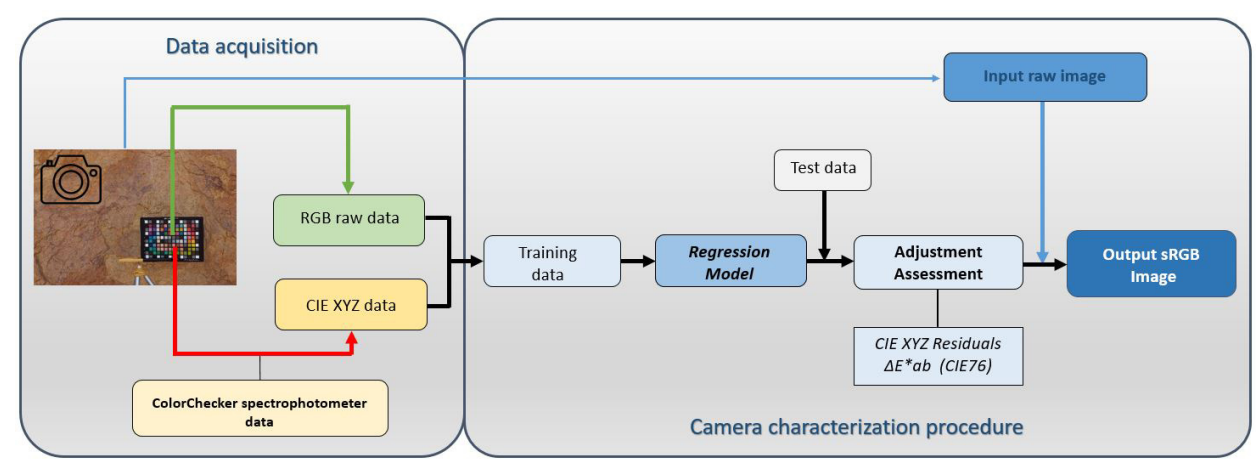

Figure 4.1: Schematic diagram designed for the camera characterization. 
To carry out the characterization, various training and test datasets are required. An important aspect on the camera characterization process is the establishment of the working color spaces. Some of the most common color spaces used are the input RGB data and the output tristimulus coordinates (Westland et al., 2012). In the preliminary stages of the study, four different transformations between color spaces were tested, including RGB-CIE XYZ, RGB-CIELAB, LMS-CIE XYZ, and LMS-CIELAB. The transformation that worked the best was the RGB-CIE XYZ, whose results are reported below in this chapter.

On the other hand, the digital RGB values are available after a complex process driven by the built-in software and electronics of the camera (Ramanath et al., 2005). Usually, a set of pre-processing operations, e.g. demosaicing, white balance, gamut mapping, color enhancement, or compression, are applied automatically to the RAW image (Figure 4.2). It is thus preferable to work with RAW data versus RGB processed or compressed image files.

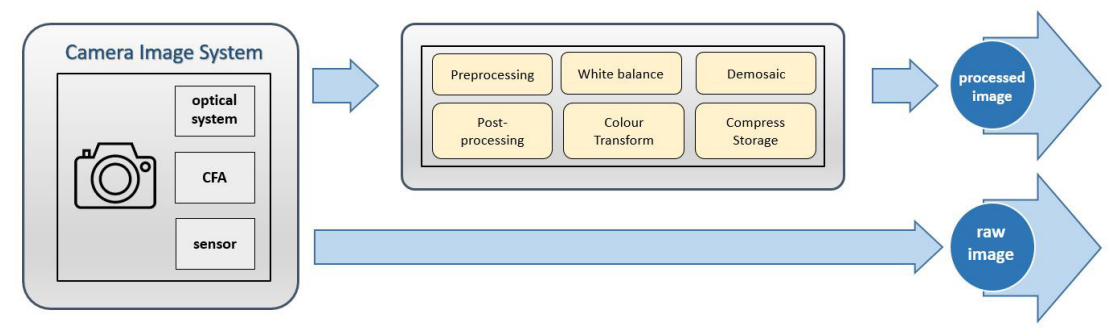

Figure 4.2: RAW images versus processed images workflow.

The RAW RGB training and test sample data were extracted from the images using our software pyColourimetry which was developed in previous research. This software was written in the Python programming language following the CIE recommendations. It allows RAW RGB data extraction from conventional camera formats and implements other colorimetric functions such as transformation among color spaces, color difference calculation, or spectral data treatment (Molada-Tebar et al., 2017).

Also, the data acquisition includes the direct measurement of the tristimulus values of the patches present in the color chart and the RAW RGB data extraction from the digital image. Thus, a color chart has to be included as a colorimetric reference in the photographic shot to carry out the camera characterization. For this experiment, we used an X-Rite ColorChecker SG Digital Color Chart as a color standard. This chart is routinely used in digital photography for color calibration. It consists of an array of 140 color patches. The number of patches is supposedly enough to cover the color domain present in the scene as well as to provide training and test data sets to analyze the results after the camera characterization.

CIE XYZ values for the ColorChecker patches must be known prior to undertaking the camera characterization. An accepted option is to use those tristimulus values provided by the manufacturer. Nevertheless, it is highly recommended to carry out a new measurement, preferably by means of a colorimeter or spectrophotometer, using the setup of the specific 
experiment. The spectral reflectance data were acquired using the spectrophotometer Konica Minolta CM-600d, following CIE recommendations ( $2^{\circ}$ standard observer under D65 illuminant). CIE XYZ coordinates are obtained by transforming the spectral data using well-known CIE formulae (CIE, 2018).

To visualize the tristimulus coordinates, it is necessary to perform a final transformation of the CIE XYZ values into the sRGB color space, which is compatible with most digital devices. This transformation is carried out based on the technical recommendations published by the International Electrotechnical Commission (IEC, 1998). Thus, the final outcome of the characterization consists of an sRGB output image for each regression model.

Once the digital camera is colorimetrically characterized, it can be used for the rigorous measurement of color simulating a colorimeter (Martínez-Verdú et al., 2003). Using a characterized camera, we can obtain accurate color information over complete scenes, which is a very important requirement to properly analyze rock art. The use of conventional cameras for color measurement allows researchers to take pictures with low-cost recording devices, suitable for carrying out heritage documentation tasks using noninvasive methodologies (Molada-Tebar et al., 2018).

\subsubsection{Gaussian Processes for Camera Characterization}

The main goal of camera characterization is to find the mapping function between the RGB color values and the CIE XYZ tristimulus coordinates:

$$
f: R G B \in \mathbb{R}^{3} \rightarrow X Y Z \in \mathbb{R}^{3}
$$

Commonly, this multivariate mapping function is divided into independent functions for each single XYZ tristimulus value. In this chapter, we propose a Gaussian process (GP) to estimate these functions, with different model parameters, $\theta_{1}, \theta_{2}$, and $\theta_{3}$, for each mapping function:

$$
\begin{aligned}
& f_{1}: R G B \in \mathbb{R}^{3} \stackrel{G P\left(\theta_{1}\right)}{\longrightarrow} X \in \mathbb{R} \\
& f_{2}: R G B \in \mathbb{R}^{3} \stackrel{G P\left(\theta_{2}\right)}{\longrightarrow} Y \in \mathbb{R} \\
& f_{3}: R G B \in \mathbb{R}^{3} \stackrel{G P\left(\theta_{3}\right)}{\longrightarrow} Z \in \mathbb{R}
\end{aligned}
$$

\subsubsection{Gaussian Process Model}

A GP is a stochastic process that defines the distribution over a collection of random variables (Bernardo et al., 1998, Rasmussen and Williams, 2006). The defining property of a GP is that any finite set of random variables is jointly distributed as a multivariate normal distribution. A GP is completely characterized by its mean and co-variance functions that control the a priori behavior of the function. GP can be used as prior probability distributions for latent functions in generalized linear models (Gelman et al., 2013). However, in this study, we focus on GP in linear 
models (a normal outcome), as we can assume that the CIE XYZ color coordinates are normally distributed.

A GP for a normal outcome $\boldsymbol{y}=\left\{y_{1}, y_{2}, \ldots, y_{n} \in \mathbb{R}\right\} \in \mathbb{R}^{n}$, paired with a matrix of $D$ input variables (predictors) $X=\left\{\boldsymbol{x}_{1}, \boldsymbol{x}_{2}, \ldots, \boldsymbol{x}_{3} \in \mathbb{R}^{n}\right\} \in \mathbb{R}^{n \times D}$, consists of defining a multivariate Gaussian distribution over $\boldsymbol{y}$ conditioned on $\mathrm{X}$ :

$$
\boldsymbol{y} \mid X \sim \mathcal{N}\left(\mu(X), K(X \mid \theta)+\sigma^{2} I\right)
$$

where $\mu(X)$ is an n-vector, $K(X \mid \theta)$ is an $n \times n$ co-variance matrix, $\sigma^{2}$ is the noise variance, and $I$ the $n \times n$ diagonal identity matrix. The mean function $\mu: X \in \mathbb{R}^{n \times D} \rightarrow \mathbb{R}^{n}$ can be anything, although it is usually recommended to be a linear model or even zero. The co-variance function $K \mid \theta: X \in \mathbb{R}^{n \times D} \rightarrow \mathbb{R}^{n \times n}$ must be a positive semidefinite matrix (Neal, 1997, Rasmussen and Williams, 2006). In this work, we use the square exponential co-variance function, which is the most commonly used function of the Matérn class of isotropic co-variance functions (Rasmussen and Williams, 2006). The squared exponential co-variance function for two observed points $i$ and $j(i, j=1, \ldots, n)$ takes the form

$$
K(X, \theta)_{i j}=\alpha^{2} \exp \left(-\frac{1}{2} \sum_{d=1}^{D} \frac{1}{\ell_{d}^{2}}\left(x_{d i}-x_{d j}\right)^{2}\right)
$$

where $\theta=\{\alpha, \ell\} ; \alpha$ is the marginal variance parameter, which controls the overall scale or the magnitude of the range of values of the GP; and $\ell=\left\{\ell_{d}\right\}_{d=1}^{D}$ the lengthscale parameter, which controls the smoothness of the function in the direction of the $d$-predictor, so that the larger the lengthscale the smoother the function.

\subsubsection{Bayesian Inference}

Bayesian inference is based on the joint posterior distribution $p\left(\theta, \sigma^{2} \mid \boldsymbol{y}, X\right)$ of parameters given the data, which is proportional to the product of the likelihood and prior distributions,

$$
p\left(\theta, \sigma^{2} \mid \boldsymbol{y}, X\right) \propto p\left(\boldsymbol{y} \mid \theta, \sigma^{2}, X\right) p(\theta) p\left(\sigma^{2}\right)
$$

In the previous equation,

$$
p\left(\boldsymbol{y} \mid \theta, \sigma^{2}, X\right)=\prod_{i} \mathcal{N}\left(y_{i} \mid 0, K_{i i}(X \mid \theta)+\sigma^{2}\right)
$$

is the likelihood of the model, where the mean function $\mu(X)$ has been set to zero for the sake of 
simplicity, and

$$
\begin{aligned}
p(\alpha) & =\mathcal{N}(\alpha \mid 0,10) \\
p(\ell) & =\prod_{d=1}^{D} \operatorname{Gamma}\left(\ell_{d} \mid 1,0.1\right) \\
p\left(\sigma^{2}\right) & =\mathcal{N}^{+}\left(\sigma^{2} \mid 0,10\right\}
\end{aligned}
$$

are the prior distributions of the parameters of the model. These correspond to weakly informative prior distributions based on prior knowledge about the magnitude of the parameters.

Predictive inference for new function values $\tilde{\boldsymbol{y}}$ for a new sequence of input values $\tilde{X}$ can be computed by integrating over the joint posterior distribution

$$
p(\tilde{\boldsymbol{y}} \mid \boldsymbol{y})=\int p\left(\tilde{\boldsymbol{y}} \mid \theta, \sigma^{2}, \tilde{X}\right) p\left(\theta, \sigma^{2} \mid \boldsymbol{y}, X\right) d \theta d \sigma^{2}
$$

To estimate both the parameter posterior distribution and the posterior predictive distribution for this model, simulation methods and/or distributional approximations methods (Gelman et al., 2013) must be used. Simulation methods based on MCMC (Brooks et al., 2011) are general sampling methods to obtain samples from the joint posterior distribution. For quick inferences and large data sets, where iterative simulation algorithms are too slow, modal and distributional approximation methods can be more efficient and approximate alternatives.

\subsubsection{Second-Order Polynomial Model}

This is the most extended model in practical camera characterization. The $N$-dimensional collections of random observations $\boldsymbol{X}, \boldsymbol{Y}$, and $\boldsymbol{Z}$ are the CIE color variables, where $X_{i}, Y_{i}$, and $Z_{i}$ represent the color coordinates of the $i$ th order observation $i(i=1, \cdots, N)$. Each $\boldsymbol{X}$, $\boldsymbol{Y}$, and $\boldsymbol{Z} N$-dimensional variable is considered to follow a normal distribution depending on an underlying second-order polynomial function $\boldsymbol{f}$ and noise variance $\sigma^{2}$,

$$
\begin{aligned}
p\left(\boldsymbol{X} \mid \boldsymbol{f}_{x}, \sigma_{x}\right) & =\mathcal{N}\left(\boldsymbol{X} \mid \boldsymbol{f}_{x}, \sigma_{x}^{2} I\right) \\
p\left(\boldsymbol{Y} \mid \boldsymbol{f}_{y}, \sigma_{y}\right) & =\mathcal{N}\left(\boldsymbol{Y} \mid \boldsymbol{f}_{y}, \sigma_{y}^{2} I\right) \\
p\left(\boldsymbol{Z} \mid \boldsymbol{f}_{z}, \sigma_{z}\right) & =\mathcal{N}\left(\boldsymbol{Z} \mid \boldsymbol{f}_{z}, \sigma_{z}^{2} I\right)
\end{aligned}
$$

where $I$ is the $N \times N$ identity matrix. The latent second-order polynomials functions $\boldsymbol{f}_{x}, \boldsymbol{f}_{y}$, and $\boldsymbol{f}_{z}$ take the form

$$
\begin{aligned}
& \boldsymbol{f}_{x}=a_{0}+a_{1} \cdot \boldsymbol{R}+a_{2} \cdot \boldsymbol{G}+a_{3} \cdot \boldsymbol{B}+a_{4} \cdot \boldsymbol{R} \cdot \boldsymbol{G}+a_{5} \cdot \boldsymbol{R} \cdot \boldsymbol{B}+a_{6} \cdot \boldsymbol{G} \cdot \boldsymbol{B}+a_{7} \cdot \boldsymbol{R}^{\mathbf{2}}+a_{8} \cdot \boldsymbol{G}^{\mathbf{2}}+a_{9} \cdot \boldsymbol{B}^{\mathbf{2}} \\
& \boldsymbol{f}_{y}=b_{0}+b_{1} \cdot \boldsymbol{R}+b_{2} \cdot \boldsymbol{G}+b_{3} \cdot \boldsymbol{B}+b_{4} \cdot \boldsymbol{R} \cdot \boldsymbol{G}+b_{5} \cdot \boldsymbol{R} \cdot \boldsymbol{B}+b_{6} \cdot \boldsymbol{G} \cdot \boldsymbol{B}+b_{7} \cdot \boldsymbol{R}^{\mathbf{2}}+b_{8} \cdot \boldsymbol{G}^{\mathbf{2}}+b_{9} \cdot \boldsymbol{B}^{\mathbf{2}}(4.8)
\end{aligned}
$$




$$
\boldsymbol{f}_{z}=c_{0}+c_{1} \cdot \boldsymbol{R}+c_{2} \cdot \boldsymbol{G}+c_{3} \cdot \boldsymbol{B}+c_{4} \cdot \boldsymbol{R} \cdot \boldsymbol{G}+c_{5} \cdot \boldsymbol{R} \cdot \boldsymbol{B}+c_{6} \cdot \boldsymbol{G} \cdot \boldsymbol{B}+c_{7} \cdot \boldsymbol{R}^{2}+c_{8} \cdot \boldsymbol{G}^{\mathbf{2}}+c_{9} \cdot \boldsymbol{B}^{\mathbf{2}}
$$

where the vectors $\boldsymbol{a}=a_{1}, \ldots, a_{9}, \boldsymbol{b}=b_{1}, \ldots, b_{9}$ and $\boldsymbol{c}=c_{1}, \ldots, c_{9}$ represent the polynomial coefficients, and $\boldsymbol{R}, \boldsymbol{G}$, and $\boldsymbol{B}$ are the $N$-dimensional variables in the input RGB space.

The likelihood functions of the variables $\boldsymbol{X}, \boldsymbol{Y}$ and $\boldsymbol{Z}$ (given the coefficients $\boldsymbol{a}, \boldsymbol{b}, \boldsymbol{c}$ ), the variance parameters $\boldsymbol{\sigma}^{\mathbf{2}}=\sigma_{x}^{2}, \sigma_{y}^{2}, \sigma_{z}^{2}$, and the variables $\boldsymbol{R}, \boldsymbol{G}$ and $\boldsymbol{B}$, take the form

$$
\begin{aligned}
p\left(\boldsymbol{X} \mid \boldsymbol{a}, \sigma_{x}, \boldsymbol{R}, \boldsymbol{G}, \boldsymbol{B}\right) & =\prod_{i}^{N} \mathcal{N}\left(X_{i} \mid \boldsymbol{a}, \sigma_{x}^{2}, R_{i}, G_{i}, B_{i}\right) \\
p\left(\boldsymbol{Y} \mid \boldsymbol{b}, \sigma_{y}, \boldsymbol{R}, \boldsymbol{G}, \boldsymbol{B}\right) & =\prod_{i}^{N} \mathcal{N}\left(Y_{i} \mid \boldsymbol{b}, \sigma_{y}^{2}, R_{i}, G_{i}, B_{i}\right) \\
p\left(\boldsymbol{Z} \mid \boldsymbol{c}, \sigma_{z}, \boldsymbol{R}, \boldsymbol{G}, \boldsymbol{B}\right) & =\prod_{i}^{N} \mathcal{N}\left(Z_{i} \mid \boldsymbol{c}, \sigma_{z}^{2}, R_{i}, G_{i}, B_{i}\right)
\end{aligned}
$$

where the subscript $i$ represents the $i$ th observed value.

\subsubsection{Bayesian Inference}

The joint posterior distributions are proportional to the product of the likelihood and prior distributions:

$$
\begin{array}{r}
p\left(\boldsymbol{a}, \sigma_{x}^{2} \mid \boldsymbol{X}\right) \propto p\left(\boldsymbol{X} \mid \boldsymbol{a}, \sigma_{x}^{2}, \boldsymbol{R}, \boldsymbol{G}, \boldsymbol{B}\right) p(\boldsymbol{a}) p\left(\sigma_{x}^{2}\right) \\
p\left(\boldsymbol{b}, \sigma_{y}^{2} \mid \boldsymbol{Y}\right) \propto p\left(\boldsymbol{Y} \mid \boldsymbol{b}, \sigma_{y}^{2}, \boldsymbol{R}, \boldsymbol{G}, \boldsymbol{B}\right) p(\boldsymbol{b}) p\left(\sigma_{y}^{2}\right) \\
p\left(\boldsymbol{c}, \sigma_{z}^{2} \mid \boldsymbol{Z}\right) \propto p\left(\boldsymbol{Z} \mid \boldsymbol{c}, \sigma_{z}^{2}, \boldsymbol{R}, \boldsymbol{G}, \boldsymbol{B}\right) p(\boldsymbol{c}) p\left(\sigma_{z}^{2}\right)
\end{array}
$$

We set vague prior distributions $p(\boldsymbol{a})=\mathcal{N}(\boldsymbol{a} \mid 0,1000), p(\boldsymbol{b})=\mathcal{N}(\boldsymbol{b} \mid 0,1000), p(\boldsymbol{c})=$ $\mathcal{N}(\boldsymbol{c} \mid 0,1000)$, and $p(\boldsymbol{\sigma})=\mathcal{N}^{+}(\sigma \mid 0,1)$ for the hyperparameters $\boldsymbol{a}, \boldsymbol{b}, \boldsymbol{c}$, and $\boldsymbol{\sigma}$, respectively, based on prior knowledge about the magnitude of the parameters.

Predictive inference for new function values $\tilde{\boldsymbol{X}}, \tilde{\boldsymbol{Y}}$, and $\tilde{\boldsymbol{Z}}$ for a new sequence of input values $\tilde{\boldsymbol{R}}, \tilde{\boldsymbol{G}}$, and $\tilde{\boldsymbol{B}}$ can be computed by integrating over the joint posterior distributions

$$
\begin{aligned}
p(\tilde{\boldsymbol{X}} \mid \boldsymbol{X}) & =\int p\left(\tilde{\boldsymbol{X}} \mid \boldsymbol{a}, \sigma_{x}^{2}, \tilde{\boldsymbol{R}}, \tilde{\boldsymbol{G}}, \tilde{\boldsymbol{B}}\right) p\left(\boldsymbol{a}, \sigma_{x}^{2} \mid \boldsymbol{X}\right) d \boldsymbol{a} d \sigma_{x}^{2} \\
p(\tilde{\boldsymbol{Y}} \mid \boldsymbol{Y}) & =\int p\left(\tilde{\boldsymbol{Y}} \mid \boldsymbol{b}, \sigma_{y}^{2}, \tilde{\boldsymbol{R}}, \tilde{\boldsymbol{G}}, \tilde{\boldsymbol{B}}\right) p\left(\boldsymbol{b}, \sigma_{y}^{2} \mid \boldsymbol{Y}\right) d \boldsymbol{b} d \sigma_{y}^{2} \\
p(\tilde{\boldsymbol{Z}} \mid \boldsymbol{Z}) & =\int p\left(\tilde{\boldsymbol{Z}} \mid \boldsymbol{c}, \sigma_{z}^{2}, \tilde{\boldsymbol{R}}, \tilde{\boldsymbol{G}}, \tilde{\boldsymbol{B}}\right) p\left(\boldsymbol{c}, \sigma_{z}^{2} \mid \boldsymbol{Z}\right) d \boldsymbol{c} d \sigma_{z}^{2}
\end{aligned}
$$

Simulation methods based on MCMC are used for estimating both the parameter posterior distribution and the posterior predictive distribution of these models. 


\subsubsection{Model Checking and Comparison}

For model assessment, common checking procedures of normality, magnitude and trends on the fitted and predicted residuals are used. Fitted residuals can be useful for identifying outliers or misspecified models and give us the goodness of the fit for the sampling patches. Furthermore, the performance of each model was assessed using the LOOCV approach (Stone, 1974). The LOOCV procedure has been previously used in color science multiple times (Li et al., 2009, Qian et al., 2017, Vazquez-Corral et al., 2014, Xiong et al., 2007), although its origins can be traced back to early practical statistics methods (Stone, 1974) and is routinely used in modern data science applications (Gareth et al., 2013).

In our study, the LOOCV consists of setting aside an individual patch and calculating the prediction model. Then, the predicted value is compared to its observed value which gives a measure of the model predictive accuracy. This allows obtaining an average of the predictive accuracy for unobserved patches as well as individual quality indicators for each color patch.

In addition to the residual analysis, it is required the assessment of the models using colorimetry metrics (Iturbe et al., 2018). Also, a LOOCV procedure was conducted to assess the predictive performance in terms of color differences. In classical colorimetry, color difference metrics are determined using formulas based on the CIELAB color space, such as $\Delta E_{a b}^{*}$ (Eq. 3.3), also known as the CIE76 color difference (CIE, 2018).

The CIE XYZ color space is not uniform, that is, equal distances in this space do not represent equally perceptible differences between color stimuli. Contrarily, CIELAB coordinates are nonlinear functions of CIE XYZ, and more perceptually uniform than the CIE XYZ color space (CIE, 2007, 2018). The $\Delta E_{a b}^{*}$ between the theoretical tristimulus coordinates against the predicted values are computed, which gives a measure of the model adjustment in a well-defined color metric.

\subsubsection{Induced Noise Analysis}

The radiometric response of a digital camera is the outcome of a number of factors, such as electromagnetic radiation, sensor electronics, the optical system, and so forth (Lebrun et al., 2013, Colom et al., 2014, Sur and Grédiac, 2015, Zhang et al., 2018, Naveed et al., 2019). The noise present on a single image is basically composed of two components: the photoresponse noise of every sensor element (pixel) and the spatial non-uniformity or fixed pattern noise of the sensor array (Riutort-Mayol et al., 2012, Marqués-Mateu et al., 2013).

The nonlinear transformation functions in camera characterization models modify the input data which are themselves affected by noise. In the camera characterization procedure, noise is transferred from the original image to the characterized image and transformed in different ways. In this research, the analysis of noise is carried out by comparing the coefficients of variation in the original and the characterized images. The noise assessment was conducted on four selected patches of the color checker $(\mathrm{C} 7, \mathrm{D} 7, \mathrm{C} 8$, and D8). 


\subsection{Results and Discussion}

\subsubsection{Model Performance Assessment}

For model assessment we processed the CIE XYZ residuals and the $\Delta E_{a b}^{*}$ color differences values after the characterization procedure.

\subsubsection{CIE XYZ Residuals}

Table 4.1 summarizes the fitted CIE XYZ and LOOCV residuals values achieved after the characterization process for the two cameras used in the study. Also, the histograms of the fitted and LOOCV residuals in both images are in Figure 4.3. Although both methodologies give satisfactory results and fit appropriately to the input RGB data, all summary statistics and histograms clearly show that GP outperforms the second-order polynomial regression model in both images. The standard deviation values, which represent the mean error, as well as the maximum and minimum residuals, are lower using the GP process than the second-order polynomial model.

Table 4.1: Fitted CIE XYZ (Res) and LOOCV residuals (RL) values after the characterization.

\begin{tabular}{|c|c|c|c|c|c|c|c|c|c|c|c|c|}
\hline & \multicolumn{12}{|c|}{ Sigma SD15 Image } \\
\hline & \multicolumn{6}{|c|}{ Gaussian Process } & \multicolumn{4}{|c|}{ Second-Order Polynomial } & \multirow{2}{*}{\multicolumn{2}{|c|}{ CIE Z }} \\
\hline & \multicolumn{2}{|c|}{ CIE X } & \multicolumn{2}{|c|}{ CIE Y } & \multicolumn{2}{|c|}{ CIE Z } & \multicolumn{2}{|c|}{ CIE X } & \multicolumn{2}{|c|}{ CIE Y } & & \\
\hline & Res & RL & Res & RL & Res & RL & Res & RL & Res & $\mathrm{RL}$ & Res & RL \\
\hline Max. & 1.88 & 4.53 & 1.80 & 4.38 & 1.28 & 3.66 & 7.02 & 7.36 & 6.87 & 7.18 & 4.80 & 5.09 \\
\hline Min. & -2.48 & -4.59 & -3.21 & -5.80 & -1.50 & -4.54 & -4.97 & -5.46 & -4.70 & -5.15 & -3.59 & -3.93 \\
\hline Std. Dev. & 0.73 & 1.39 & 0.77 & 1.48 & 0.49 & 1.01 & 1.77 & 1.92 & 1.82 & 1.98 & 1.29 & 1.38 \\
\hline
\end{tabular}

Fujifilm IS PRO Image

\begin{tabular}{lcccccccccccc}
\hline & \multicolumn{4}{c}{ Gaussian Process } & \multicolumn{4}{c}{ Second-Order Polynomial } \\
& \multicolumn{2}{c}{ CIE X } & \multicolumn{2}{c}{ CIE Y } & \multicolumn{2}{c}{ CIE Z } & \multicolumn{3}{c}{ CIE X } & \multicolumn{2}{c}{ CIE Y } & \multicolumn{2}{c}{ CIE Z } \\
& Res & RL & Res & RL & Res & RL & Res & RL & Res & RL & Res & RL \\
\hline Max. & 2.20 & 4.42 & 1.98 & 3.71 & 1.54 & 3.33 & 7.08 & 7.53 & 6.48 & 6.90 & 3.15 & 3.35 \\
Min. & -3.72 & -4.25 & -2.63 & -3.84 & -1.35 & -1.80 & -4.25 & -4.69 & -3.12 & -3.31 & -1.96 & -2.45 \\
Std. Dev. & 0.94 & 1.46 & 0.78 & 1.20 & 0.45 & 0.74 & 1.75 & 1.92 & 1.45 & 1.58 & 0.49 & 0.86 \\
\hline
\end{tabular}

Thus, given the results achieved using the GP, a notable improvement can be observed compared with the common second-order polynomial models, that is, a higher adjustment correlation coefficient and a greater predictive capacity were achieved. An improvement in the predictive capacity (LOOCV residuals) implies a better model generalization, that is, a better capacity for interpolation and extrapolation. This is a key aspect in the characterization procedure, as the output digital image is the result of the application of the model established. 
Sigma SD15 image

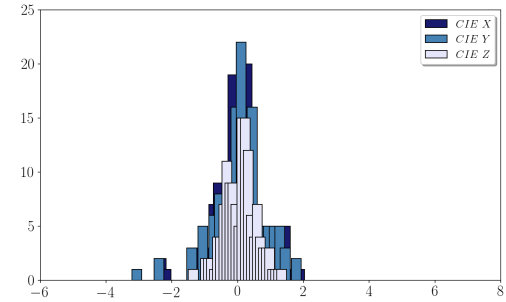

(a)

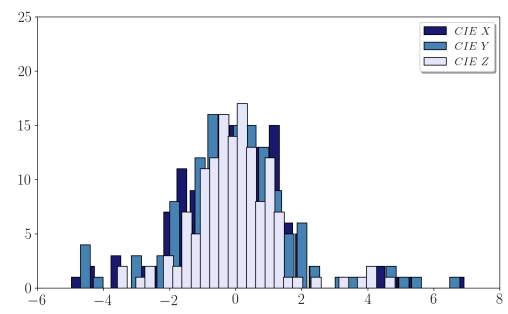

(c)

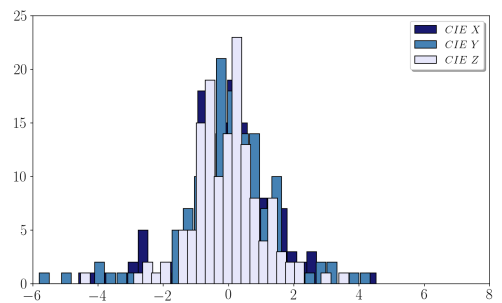

(b)

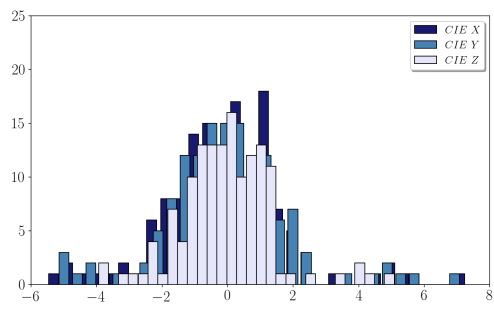

(d)

Fujifilm IS PRO image

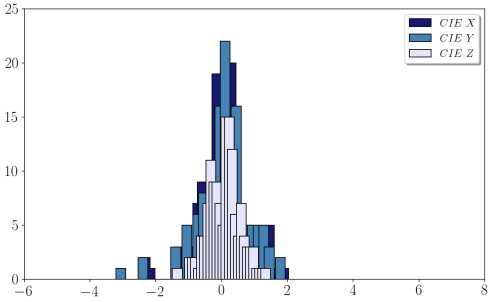

(e)

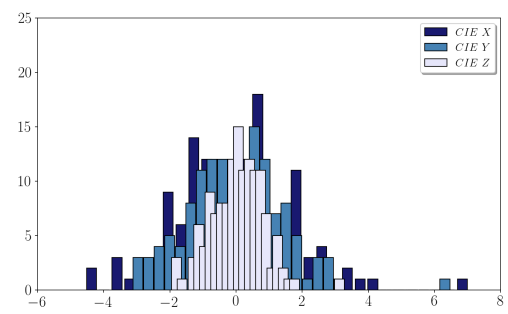

(g)

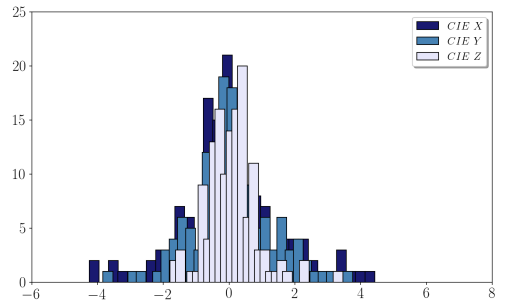

(f)

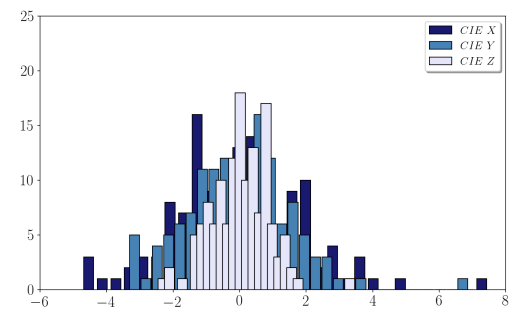

(h)

Figure 4.3: CIE XYZ residuals histograms after the adjustment: (a,b,e,f) Gaussian process; $(\mathrm{a}, \mathrm{d}, \mathrm{g}, \mathrm{h})$; Second-order polynomial; (a,c,e,g); CIE XYZ residuals; (b,d,f,h); LOOCV residuals. 


\subsubsection{2 $\Delta E_{a b}^{*}$ Color Differences}

The $\Delta E_{a b}^{*}$ color differences (Eq. 3.3) obtained between the theoretical and predicted values allowed us to assess the colorimetric quality achieved after the adjustment. The results obtained for the $\Delta E_{a b}^{*}$ are shown numerically in Table 4.2. Also, they can be observed graphically in Figure 4.4 (for the Sigma SD15) and Figure 4.5 (for the Fujifilm IS PRO), where the red line delimits the JND tolerance established in 4 CIELAB units.

Table 4.2: $\Delta E_{a b}^{*}$ summary of the statistical results after the characterization.

\begin{tabular}{lcccccccc}
\hline & \multicolumn{3}{c}{ Sigma SD15 Image } & \multicolumn{3}{c}{ Fujifilm IS PRO Image } \\
\hline & \multicolumn{3}{c}{ GP } & \multicolumn{2}{c}{ 2nd-Order Polynomial } & \multicolumn{2}{c}{ GP } & \multicolumn{2}{c}{ 2nd-Order Polynomial } \\
& $\Delta E_{a b}^{*}$ & LOOCV & $\Delta E_{a b}^{*}$ & LOOCV & $\Delta E_{a b}^{*}$ & LOOCV & $\Delta E_{a b}^{*}$ & LOOCV \\
\hline Max. & 8.251 & 8.906 & 15.086 & 17.814 & 8.213 & 8.409 & 12.634 & 13.021 \\
Mean & 1.755 & 2.440 & 2.561 & 2.751 & 1.817 & 2.285 & 2.753 & 2.958 \\
Median & 1.403 & 2.180 & 2.135 & 2.205 & 1.486 & 1.913 & 2.101 & 2.281 \\
Std. Dev. & 1.479 & 1.863 & 1.836 & 2.022 & 1.457 & 1.729 & 2.186 & 2.305 \\
\hline
\end{tabular}

Under a strict colorimetric criterion, the average and median values for $\Delta E_{a b}^{*}$ obtained using both regression models are less than 3 CIELAB units, that is, lower than the JND. However, the $\Delta E_{a b}^{*}$ color differences obtained confirm that the adjustment based on the GP model offers better results than the second-order polynomial regression for both cameras. The values achieved for the mean and median $\Delta E_{a b}^{*}$ in both images are similar using the second-order polynomial regression model.

It is clearly observed that the main improvement is in the maximum $\Delta E_{a b}^{*}$ values obtained. Although the average for LOOCV $\Delta E_{a b}^{*}$ is slightly lower using the GP, the maximum values for LOOCV $\Delta E_{a b}^{*}$ decreases significantly using this model. The maximum values obtained are 17.814 and 13.021 for the Sigma SD15 and the Fujifilm IS PRO image, respectively, using a second-order polynomial regression model, whereas the maximum values for LOOCV $\Delta E_{a b}^{*}$ using the GP are 8 CIELAB units (Table 4.2).

Moreover, the number of patches with $\Delta E_{a b}^{*}$ greater than 4 units (JND) clearly decrease for both images after applying the GP (Figures 4.4 and 4.5). Thus, the GP improvement achieved in the adjustment is noticeable in colorimetric terms, reaching lower magnitude residuals (Table 4.1) and $\Delta E_{a b}^{*}$ values (Table 4.2), which means better model fits and higher predictive characteristics. 


\section{Sigma SD15 image}
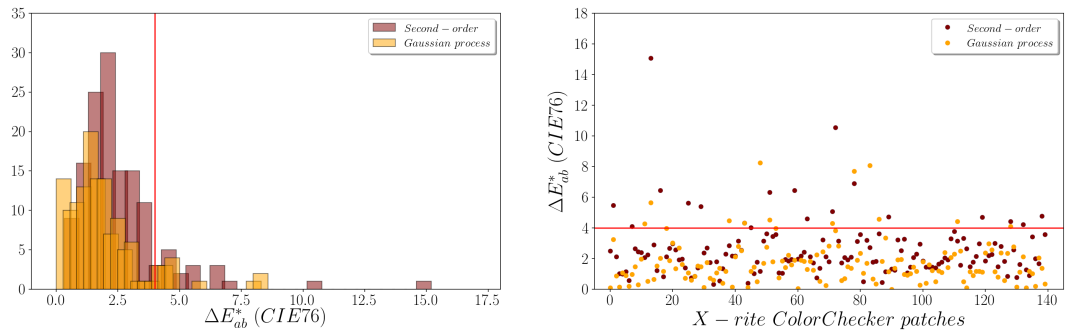

(a)
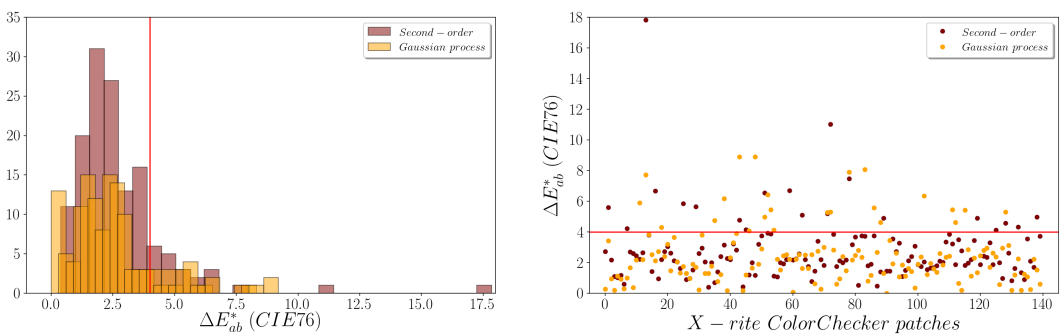

(b)

Figure 4.4: Sigma SD15 $\Delta E_{a b}^{*}$ values for the X-Rite patches: (a) $\Delta E_{a b}^{*}$; (b) LOOCV $\Delta E_{a b}^{*}$.

\section{Fujifilm IS PRO}
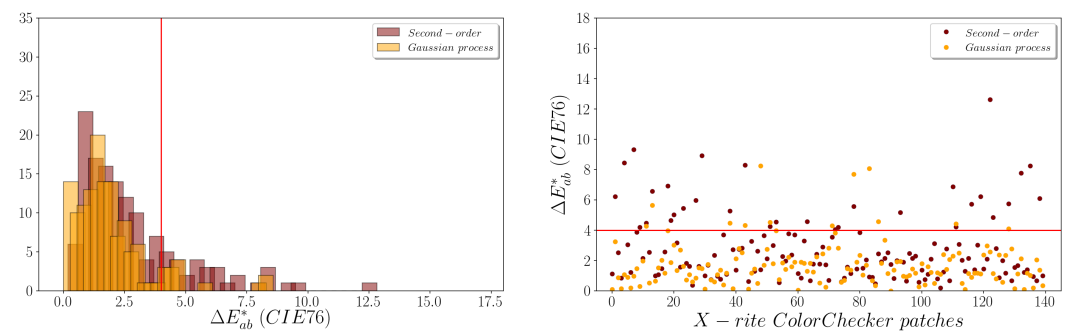

(a)
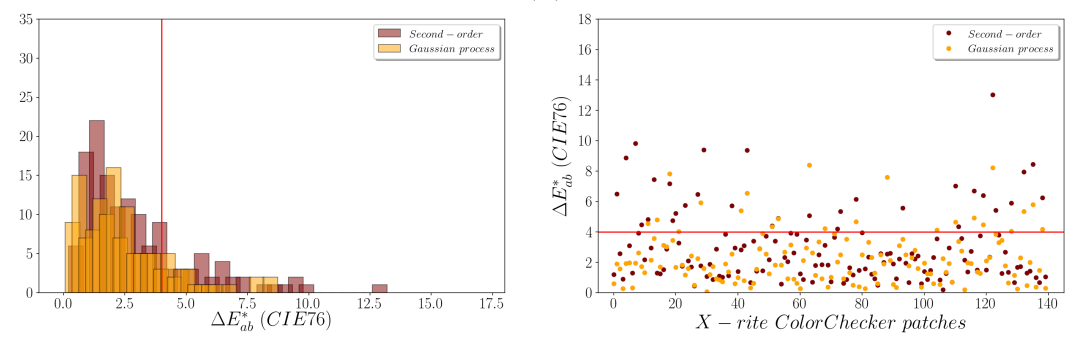

(b)

Figure 4.5: Fujifilm IS PRO $\Delta E_{a b}^{*}$ values for the X-Rite patches: (a) $\Delta E_{a b}^{*}$; (b) LOOCV $\Delta E_{a b}^{*}$. 


\subsubsection{Analysis of Color Chart Patches}

The use of the LOOCV procedure in this study is twofold: it allows an overall model checking as well as analyzing patches used in the camera characterization at individual level. Values for $\Delta E_{a b}^{*}$ less than 4 CIELAB units (JND value represented by the red line in the plots in Figures 4.4 and 4.5) are achieved for the majority of the patches. Also, it is clearly observed that the Fujifilm IS PRO camera gives better results than the Sigma SD15, particularly after applying the GP (cf. Figure 4.5 b with Figure 4.4 b).

Table 4.3 displays the percentage of patches with a LOOCV $\Delta E_{a b}^{*}$ greater than 4 CIELAB units for the different regression models performed. Once again, the GP model gives slightly better results than the second-order polynomial model, especially for the Fujifilm IS PRO digital camera.

Table 4.3: Percentage of patches with LOOCV $\Delta E_{a b}^{*}>4$ CIELAB units.

\begin{tabular}{llcccc}
\hline & & \multicolumn{2}{c}{ Sigma SD15 } & \multicolumn{2}{c}{ Fujifilm IS PRO } \\
& & Patches & \% & Patches & \% \\
\hline \multirow{2}{*}{ Model } & Gaussian process & 12 & 8.57 & 11 & 7.86 \\
& Second-order & 19 & 13.57 & 31 & 22.14 \\
LOOCV & Gaussian process & 23 & 16.43 & 22 & 15.71 \\
& Second-order & 20 & 14.29 & 31 & 22.14 \\
\hline
\end{tabular}

Particularly, there are eight patches with the highest $\Delta E_{a b}^{*}$ values in both images regardless of the model applied (A8, B4, B9, E4, G4, H3, H9, and M3). These patches can be easily identified on the X-rite ColorChecker (Figure 4.6 a) as well as on the CIE chromaticity diagram (Figure $4.6 \mathrm{~b}$ ). The worst results are found in patches E4, H9, B4, and G4 (blue, green, purple, and red, respectively). Note that patches E4 (blue), G4 (red), and H9 (green) are near the vertices of the triangle that delimits the color gamut, that is the chromatic domain, of the sRGB color space (white line plotted in Figure 4.6 b).

Thus, nature and colorimetric characteristics of the color patches used as training sample set have an effect on the overall accuracy of the model used (Chou et al., 2013). The colors represented with the highest $\Delta E_{a b}^{*}$ values in these patches correspond to saturated colors that are commonly found in artificial or industrial objects, but not in natural scenes such as those found in archaeological applications. We have to keep in mind that, usually, color charts used as colorimetric reference are designed mainly for industrial processes or photographic applications. Therefore, purple (B4, H3, and M3), blue (E4), green (H9 and B9), and bright red (G4) colors will not likely be present in archaeological scenes. Consequently, these color patches could be removed from the training sample during the characterization process without affecting the global accuracy. 


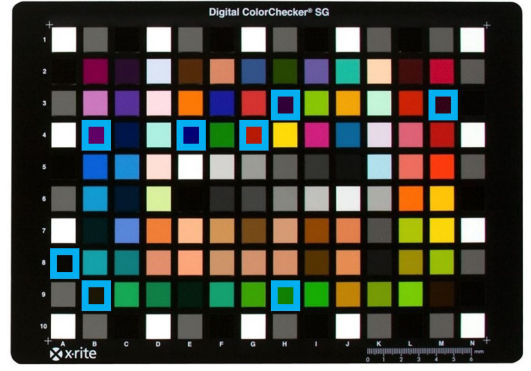

(a)

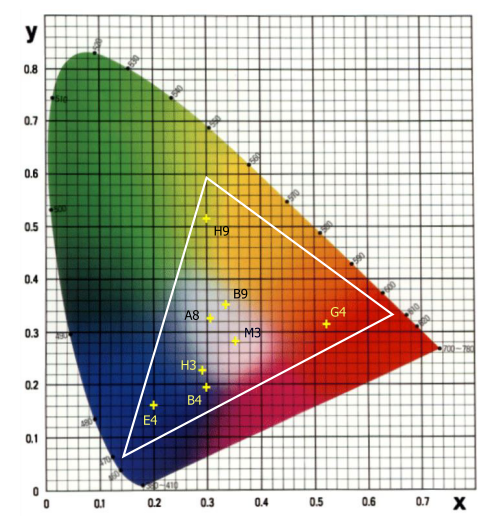

(b)

Figure 4.6: Patches with higher LOOCV $\Delta E_{a b}^{*}$ values: (a) on X-Rite ColorChecker; (b) on CIE chromaticity diagram.

Previous research shows that a proper selection of the patches, such as the skin tone colors, provides suitable results for camera characterization procedure applied in rock art paintings (Shen et al., 2007, Molada-Tebar et al., 2019a). In Spanish Levantine rock art paintings, it is more frequent to find reddish or black colors (only dark reds in the "Cova dels Cavalls") in pigments and skin tone or brown colors in the support. It is clearly observed that these patches work correctly regardless of the regression model used.

\subsubsection{Induced Noise Results}

The two cameras used in this study have different built-in sensors. The Sigma SD15 camera incorporates a Foveon@X3 sensor, whereas the Fujifilm IS PRO carries a Super CCD sensor. The values of the variation coefficients were computed and compared between the input RGB image and the output sRGB characterized image for the two mathematical transformations. The pixel variability evaluation was conducted in a reduced group of ColorChecker patches with homogeneous reflectance (C7, D7, C8, and D8).

Table 4.4 shows the variation coefficients of the RAW RGB digital values from the original images before the camera characterization (Figure $4.7 \mathrm{a}$, e). It is informative to contrast these values with the variation coefficients obtained for the CIE XYZ (Table 4.5) and sRGB transformed data (Table 4.6) respectively. For a brief overview, Table 4.7 shows a summary of the variation coefficients obtained.

Moreover, as the degree of the polynomial model used can affect the results achieved in terms of noise, we included the comparison of the variation coefficients for the linear model as well (Yamakable et al., 2017). Our outcomes show basically the same results in the second-order and linear models, which are still slightly worse than the GP result (cf. Tables 4.5 and 4.6). The trend found in the induced noise results, was that noise depends on the sensor and therefore it is different for each camera regardless of the mathematical model. 
The coefficients obtained for the digital values on the original image indicate that the noise generated directly by the Foveon $\mathbb{R}$ X3 sensor is greater than the noise in the SuperCCD (Table 4.4). Apparently, the SuperCCD sensor seems to respond better in the RAW data collection stage in terms of noise variability.

Also, the overall results confirm the better performance of the SuperCCD when compared with the Foveon $\mathrm{R}$ X3 sensor (cf. Tables 4.5 and 4.6). Table 4.7 shows that none of the regression models applied to the Fujifilm IS PRO image increased the original variability coefficients. In fact, the coefficients decrease slightly after applying the GP; with the opposite behavior present in the

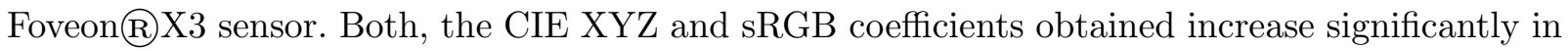
the Foveon $\mathrm{R}$ X3 sensor, regardless of the characterization model used.

Table 4.4: Noise variation coefficients of the original RGB digital numbers.

\begin{tabular}{lllllll}
\hline & \multicolumn{3}{l}{ Sigma SD15 Image } & \multicolumn{3}{c}{ Fujifilm IS PRO Image } \\
Patch & R & G & B & R & G & B \\
\hline C7 & 0.020488 & 0.014114 & 0.009923 & 0.011586 & 0.01077 & 0.01223 \\
C8 & 0.036863 & 0.019129 & 0.017545 & 0.013394 & 0.01170 & 0.00851 \\
D7 & 0.018434 & 0.016139 & 0.016432 & 0.008498 & 0.01294 & 0.01039 \\
D8 & 0.015118 & 0.014687 & 0.015908 & 0.010909 & 0.01162 & 0.00933 \\
\hline
\end{tabular}

Table 4.5: Variation coefficients of the output CIE XYZ coordinates.

\begin{tabular}{|c|c|c|c|c|c|c|c|c|c|}
\hline & \multicolumn{9}{|c|}{ Sigma SD15 Image } \\
\hline & \multicolumn{3}{|c|}{ Gaussian Process } & \multicolumn{3}{|c|}{ Second-Order Polynomial } & \multicolumn{2}{|l|}{ Linear } & \multirow[b]{2}{*}{$\mathbf{Z}$} \\
\hline Patch & $\mathbf{X}$ & $\mathbf{Y}$ & $\mathrm{Z}$ & $\mathbf{X}$ & $\mathbf{Y}$ & $\mathbf{Z}$ & $\mathbf{X}$ & $\mathbf{Y}$ & \\
\hline $\mathrm{C} 7$ & 0.03673 & 0.01413 & 0.03559 & 0.02447 & 0.02620 & 0.03129 & 0.01988 & 0.04851 & 0.03192 \\
\hline $\mathrm{C} 8$ & 0.01290 & 0.06014 & 0.09700 & 0.01360 & 0.05988 & 0.09379 & 0.01916 & 0.04617 & 0.10635 \\
\hline D7 & 0.05272 & 0.05006 & 0.05985 & 0.07844 & 0.03741 & 0.06928 & 0.03755 & 0.05664 & 0.06164 \\
\hline \multirow[t]{3}{*}{ D8 } & 0.01933 & 0.04773 & 0.08251 & 0.01365 & 0.05254 & 0.07137 & 0.01632 & 0.04362 & 0.07257 \\
\hline & \multicolumn{9}{|c|}{ Fujifilm IS PRO Image } \\
\hline & \multicolumn{3}{|c|}{ Gaussian Process } & \multicolumn{3}{|c|}{ Second-Order Polynomial } & Linear & & \\
\hline Patch & $\mathbf{X}$ & $\mathbf{Y}$ & $\mathbf{Z}$ & $\mathrm{X}$ & $\mathbf{Y}$ & $\mathrm{Z}$ & $\mathbf{X}$ & $\mathbf{Y}$ & $\mathrm{Z}$ \\
\hline $\mathrm{C} 7$ & 0.00824 & 0.01098 & 0.00967 & 0.00905 & 0.01275 & 0.01009 & 0.01037 & 0.01197 & 0.00969 \\
\hline C8 & 0.01054 & 0.01162 & 0.01730 & 0.01104 & 0.01179 & 0.01782 & 0.00983 & 0.01078 & 0.01831 \\
\hline D7 & 0.01058 & 0.01286 & 0.01040 & 0.00929 & 0.01163 & 0.01066 & 0.00812 & 0.01156 & 0.00994 \\
\hline D8 & 0.00552 & 0.01033 & 0.01639 & 0.00653 & 0.01080 & 0.01632 & 0.00618 & 0.00916 & 0.01390 \\
\hline
\end{tabular}


Table 4.6: Variation coefficients of the output sRGB digital numbers.

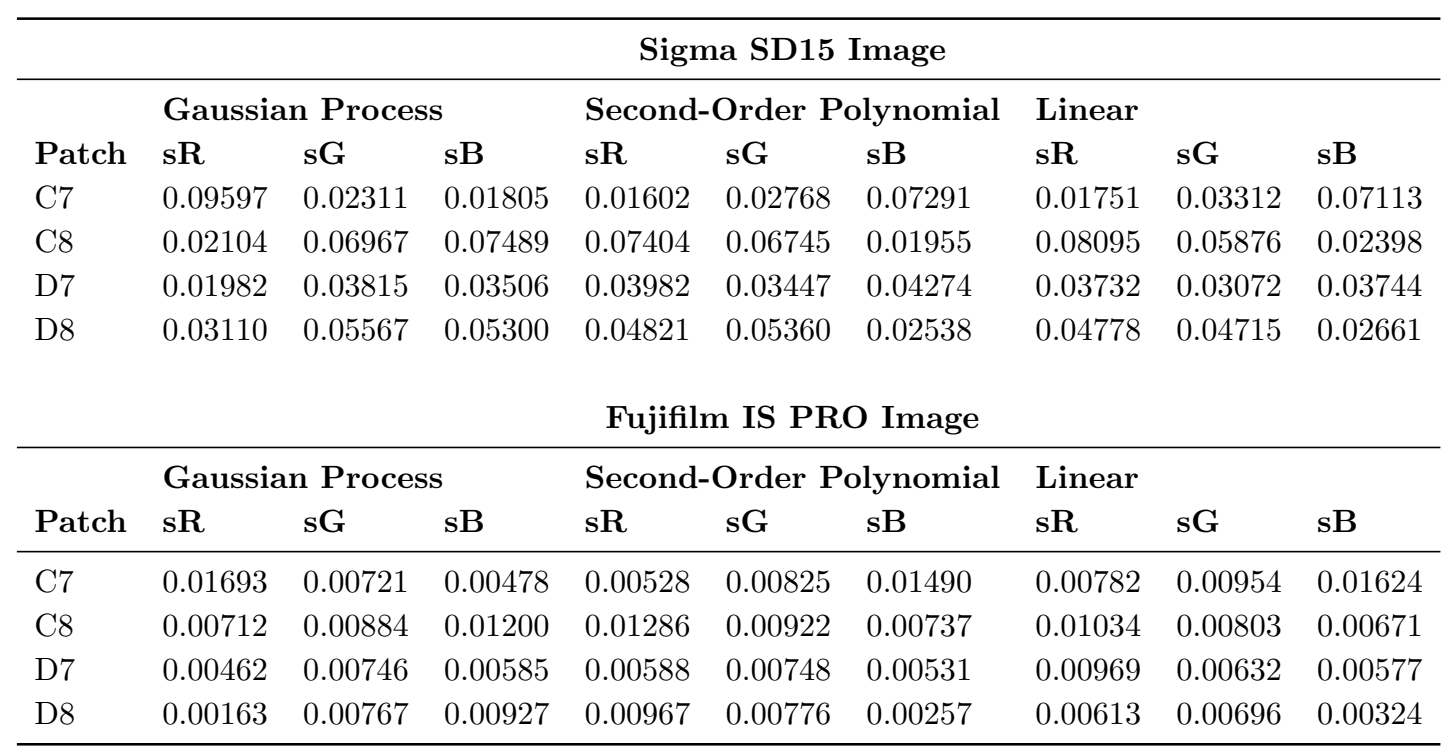

Table 4.7: Summary of the variation coefficients obtained.

\begin{tabular}{llll}
\hline & & $\begin{array}{l}\text { Sigma } \\
\text { SD15 Image }\end{array}$ & $\begin{array}{l}\text { Fujifilm } \\
\text { IS PRO Image }\end{array}$ \\
\hline Original & RGB & 0.01790 & 0.01099 \\
Gaussian process & CIE XYZ & 0.04739 & 0.01120 \\
& sRGB & 0.04463 & 0.00778 \\
Second-order & CIE XYZ & 0.04766 & 0.01148 \\
Linear & sRGB & 0.04349 & 0.00810 \\
& CIE XYZ & 0.04774 & 0.01082 \\
& sRGB & 0.04271 & 0.00807 \\
\hline
\end{tabular}

Additionally, Figure 4.7 displays the comparative noise images as a result of the different color space transforms during the camera characterization. Greater noise is produced by the Foveon $\mathrm{R}$ X3 sensor versus the SuperCCD sensor. It is evident that the best results are obtained for the Fujifilm IS PRO camera in terms of noise (cf. Figure $4.7 \mathrm{~b}-\mathrm{d}, \mathrm{f}-\mathrm{h}$ ). The architecture, characteristics and operation of each sensor are different, and therefore, the image noise effects differ depending on the camera used. From these results, it turns out that the digital camera selected for photographic work is a crucial aspect to be taken into account in archaeological applications. 


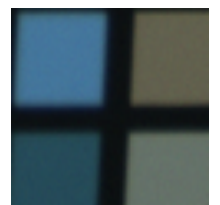

(a)

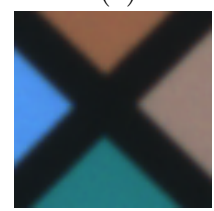

(e)

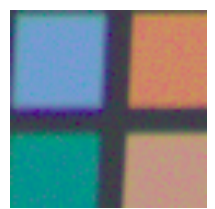

(b)

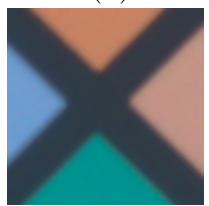

(f)

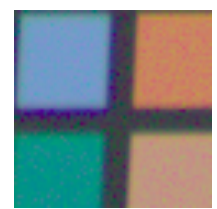

(c)

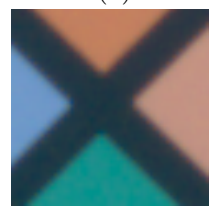

(g)

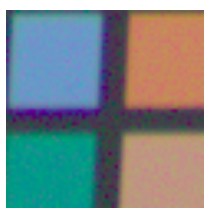

(d)

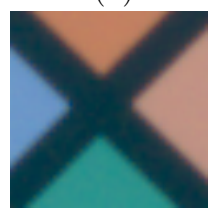

(h)

Figure 4.7: Noise comparative detail sRGB images after characterization: (a-d) Sigma SD15; (e-h) FujifilmIS PRO; (a,e) Original RAW images; $(b, f)$ GP; $(\mathrm{c}, \mathrm{g})$ Second-order polynomial model; $(\mathrm{d}, \mathrm{h})$ Linear model.

\subsubsection{Output sRGB Characterized Images}

The original RAW images were successfully transformed into the same device-independent color space by means of the two regression models applied (Figure 4.8). Both the GP and the second-order polynomial model gave similar results. Even an experienced observer is unable to perceive differences between the images generated with the two regression models applied for both SLR digital cameras (cf. Figure $4.8 \mathrm{c}-\mathrm{f}$ ). The JND threshold of 4 CIELAB units is suitable in most practical applications (specifically in this study), and proves that human vision cannot perceive the improvement obtained with the GP (cf. $\Delta E_{a b}^{*}$ GP and second-order polynomial mean values in Table 4.2).

\subsection{3 $\Delta E_{a b}^{*}$ Mapping Images}

To verify the colorimetric quality achieved using both the GP and the polynomial regression model, we mapped the $\Delta E_{a b}^{*}$ between the two characterized sRGB images obtained (Figure 4.9). The predominant green color $\left(\Delta E_{a b}^{*}<2\right.$ units) observed in the mapping images shows that the results obtained are very satisfactory regardless of the model applied. Again, the best results were obtained for the Fujifilm IS PRO image (Figure 4.9 b). Nevertheless, for common applications, both regression models can be used since they offer successful results.

The detail of the color chart shown in Figure 4.9 displays some patches marked in red, that is, with $\Delta E_{a b}^{*}$ color differences values greater than 4 CIELAB units (JND). The red color is also found on the edge of the ColorChecker. Indeed, it was on the color chart background support where we found most of the red pixels. It is well known that color depends on the incident lighting; thus, changes in geometry produce local changes of illumination in some parts of the scene. This means that in certain areas the initial homogeneous lighting hypothesis is not fulfilled, and the regression model does not fit well to the input data in shaded areas. This circumstance also reflects the importance of illumination in colorimetry. 


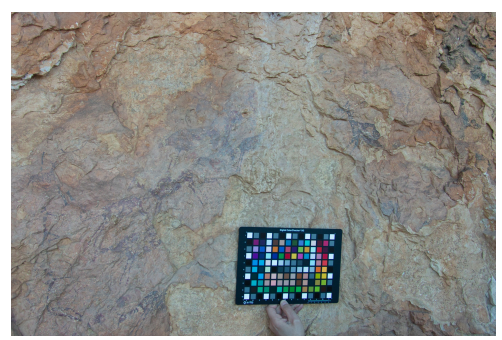

(a)

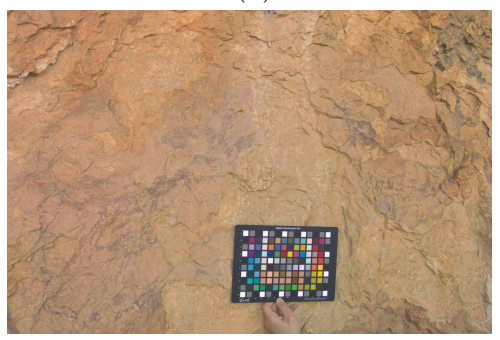

(c)

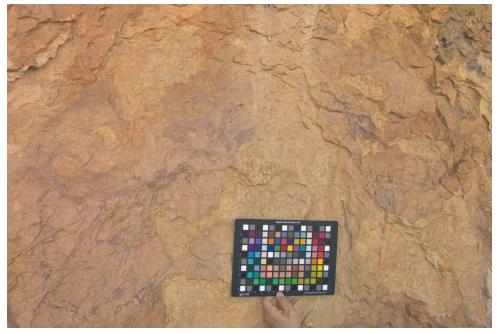

(e)

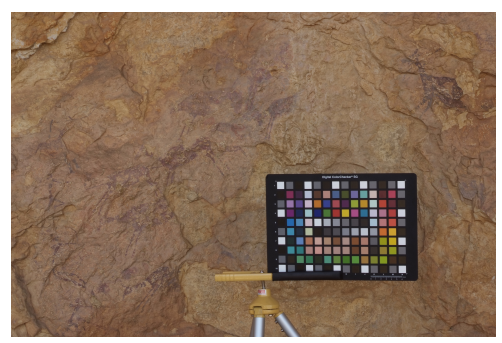

(b)

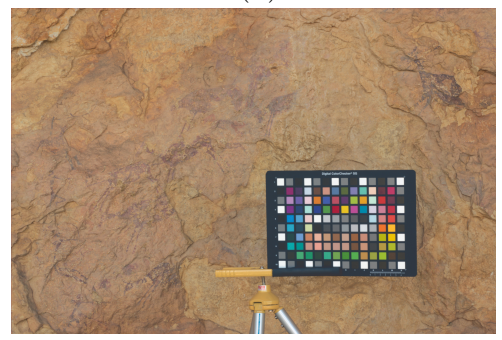

(d)

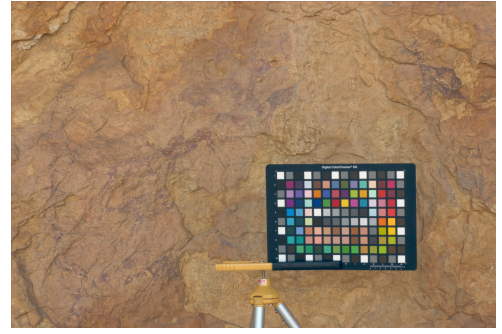

(f)

Figure 4.8: Original and output sRGB characterized images: (a,c,e) Sigma SD15; (b,d,f) Fujifilm IS PRO; (a,b) Original; (c,d) GP; (e,f) Second-order polynomial model.

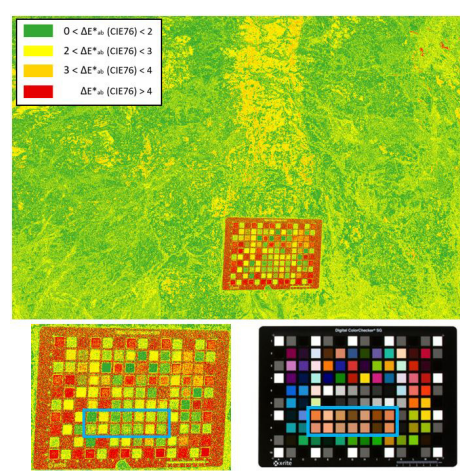

(a)

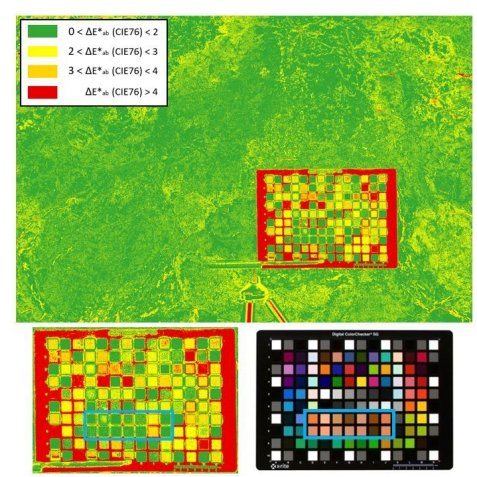

(b)

Figure 4.9: $\Delta E_{a b}^{*}$ difference mapping images between GP and second-order polynomial characterization models: (a) Sigma SD15; (b) Fujifilm IS PRO. 


\subsubsection{Rock Art Specimen Detail Images}

We also compared the results obtained in two different rock art details present on the scene: the wounded animal detail (right upper corner); and the hunting scene (lower left corner) (Figure 4.10). For each detail selected from the scene, we show the clip of the original image, the output (characterized) image after applying the different regression models, as well as the color difference mappings between both models. In order to facilitate the identification of the specimens, a mask has been applied to the latter image (Figures 4.11 and 4.12).

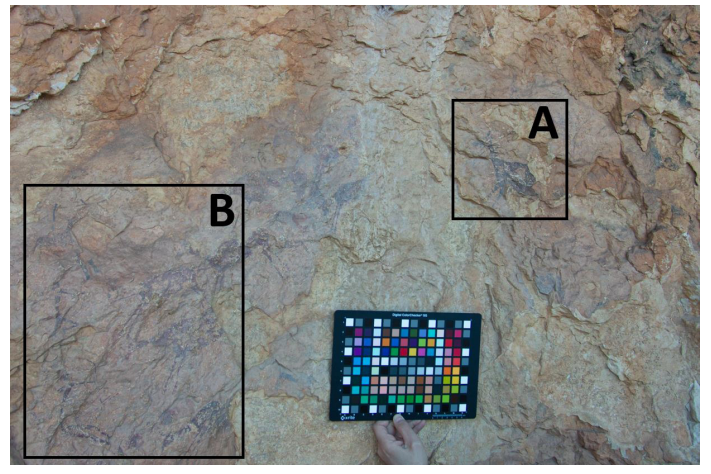

(a)

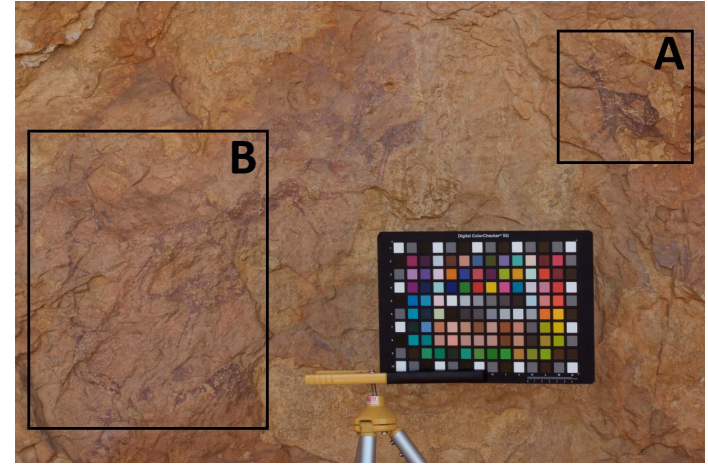

(b)

Figure 4.10: Selected rock art scenes: (a) SigmaSD15; (b) Fujifilm IS PRO. (A) Wounded animal detail. (B) Hunting scene.

Better results are observed in the images characterized by the Fujifilm IS PRO. The color differences $\Delta E_{a b}^{*}$ obtained for the pigments were under 2 CIELAB units, hence the predominantly green color (Figures $4.11 \mathrm{~g}$ and $4.12 \mathrm{~g}$ ). A limited set of pixels marked in red $\left(\Delta E_{a b}^{*}>4\right.$ CIELAB units) are observed in areas where the homogeneous lighting hypothesis is not fulfilled due to geometry changes in the support (Figure $4.11 \mathrm{~g})$. On the other hand, the yellow values $\left(\Delta E_{a b}^{*}\right.$ 2-3 units) present in the Sigma SD15 image can be due to the fact that the GP slightly improves the camera characterization (Figures $4.11 \mathrm{c}$ and $4.12 \mathrm{c}$ ). Therefore, it is important to previously make a correct selection of the camera to be used in the characterization process for archaeological research.

It can be seen that the images have been successfully characterized, independently of the regression model used. All details in the characterized images present the same ranges of colors, as well as homogeneous lighting for both cameras (cf. Figure 4.11 b, d, f, h; Figure 4.12 b, d, f, h). 


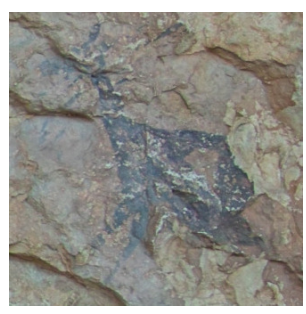

(a)

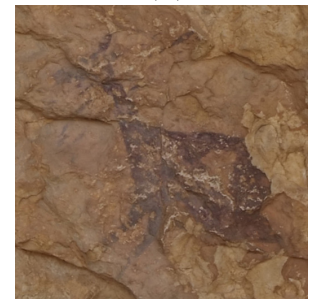

(e)

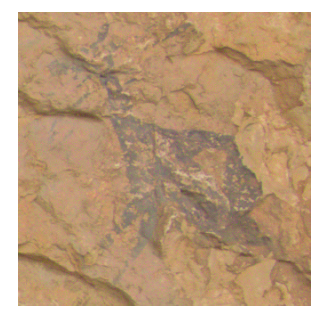

(b)

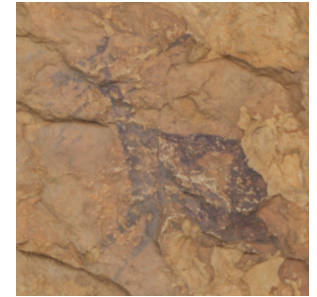

(f)

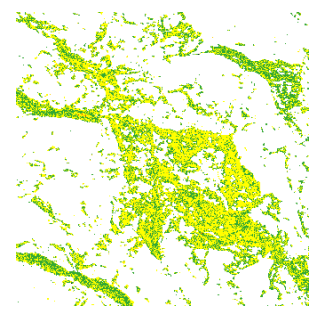

(c)

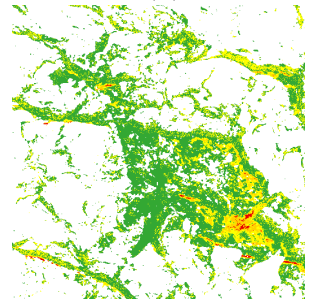

(g)

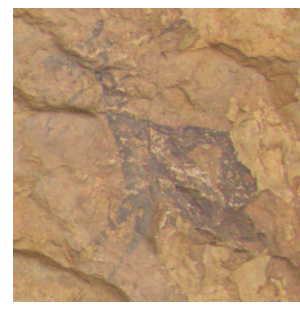

(d)

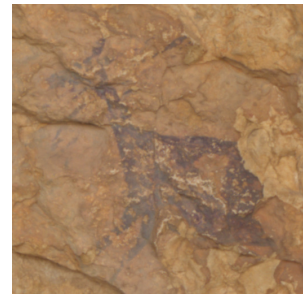

(h)

Figure 4.11: Wounded animal images: (a-d) Sigma SD15; (e-h) Fujifilm IS PRO; (a,e) Original image; (b,f) GP characterized image; (c,g) $\Delta E_{a b}^{*}$ comparative image; (d,h) Second-order characterized image.

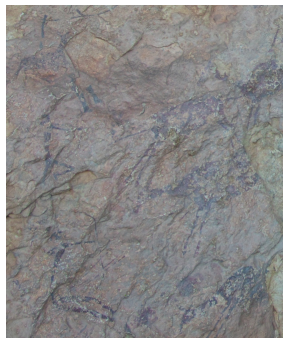

(a)

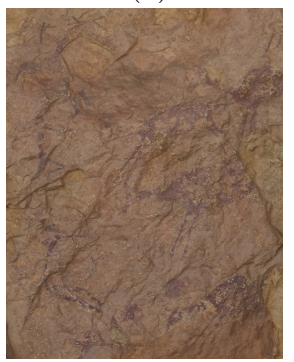

(e)

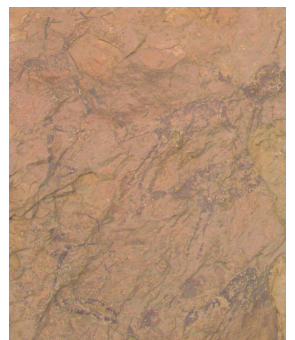

(b)

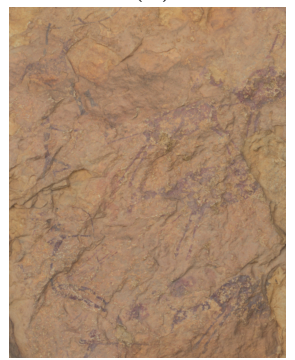

(f)

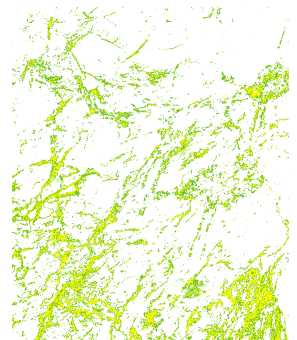

(c)

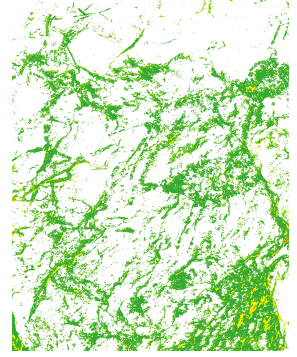

(g)

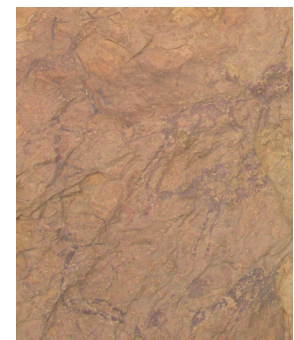

(d)

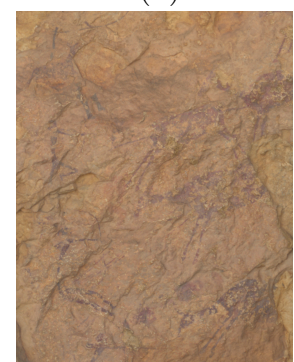

(h)

Figure 4.12: Hunter scene images: (a-d) Sigma SD15; (e-h) Fujifilm IS PRO; (a,e) Original image; $(\mathrm{b}, \mathrm{f})$ GP characterized image; (c,g) $\Delta E_{a b}^{*}$ comparative image; (d,h) Second-order characterized image. 


\subsection{Conclusions}

The use of digital images to support cultural heritage documentation techniques has undergone unprecedented advances in the last decades. However, the original RGB data provided by digital cameras cannot be used for rigorous color measurement and communication. To face the lack of colorimetric rigor of the input RGB data recorded by the sensor, it is necessary to conduct a colorimetric camera characterization; alternatively, color profiles can also be used.

In this study, the experimental assessment of a GP model has been carried out, and compared with a common second-order polynomial model. Although both regression models yielded good results, the use of the GP provides an improvement in colorimetric terms and fits better with the original RAW RGB data. The lowest CIE XYZ residual values achieved for the adjustment and $\Delta E_{a b}^{*}$ color differences supports the use of a GP as a proper model for characterizing digital cameras. However, for practical purposes, the final sRGB characterized images derived from both the GP and the second-order polynomial model can be used with success in cultural heritage documentation and preservation tasks.

Additionally, the GP regression model has been tested on two SLR digital cameras with different built-in sensors to analyze the performance of the model in terms of pixel variability. The noise errors achieved show that similar results were obtained regardless of the regression model used. However, the results also reveal that the induced noise highly depends on the camera sensor, which is clearly significant in the Foveon $\mathrm{R}$ X3 but not in the Super CCD. Thus, the correct choice of the digital camera is a key factor to be taken into consideration in archaeology surveys.

It is observed that the camera characterization procedure allows clear identification of the different pigments used in the scene, a proper separation from the support, the achievement of more accurate digital tracings, and accurate color measurement for monitoring aging effects on pigments. This methodology proves to be highly applicable not only in cultural heritage documentation tasks, but in any scientific and industrial discipline where a correct registration of the color is required. 


\section{5 \\ Patch Adaptive Selection with $K$-Means (P-ASK)}

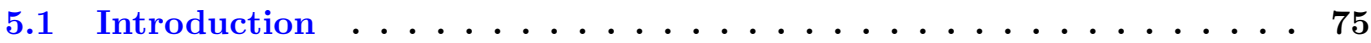

5.2 Materials and Methods . . . . . . . . . . . . 76

5.2 .1 Data set acquisition . . . . . . . . . . . . . . 76

5.2 .2 Colorimetric camera characterization procedure . . . . . . . . 77

5.2 .2 .1 Training data . . . . . . . . . . . . . 78

5.2.2.2 Regression model and accuracy assessment . . . . . . . . 78

5.2.2.3 Output sRGB characterized image . . . . . . . . . . . 79

$5.2 .3 \quad K$-means clustering technique . . . . . . . . . . . . . . . 79

5.2.3.1 Optimal $K$ number of clusters . . . . . . . . . . . 80

5.2.3.2 Clustering Algorithm . . . . . . . . . . . . . . 80

5.2.3.3 Initial starting conditions . . . . . . . . . . . . . . . 81

5.2.4 Color patch adaptive selection with $K$-means (P-ASK) $\ldots \ldots \ldots . . .81$

5.2.4.1 $K$-means dominant color extraction . . . . . . . . . 82

5.2.4.2 Identification of dominant color patches . . . . . . . . . 83

5.3 Results . . . . . . . . . . . . . . . . . . 84

5.3.1 Setting the optimal parameters for the $K$-means clustering algorithm . 84

$5.3 .1 .1 \quad K$ number of clusters $\ldots \ldots \ldots \ldots \ldots$

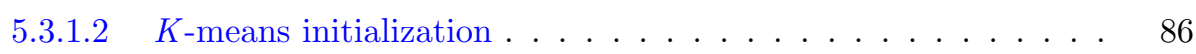

5.3.1.3 Identification of dominant color patches . . . . . . . . . 87

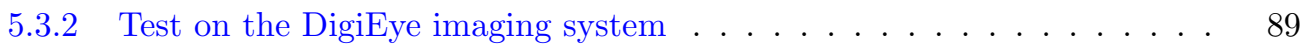

5.3.3 Assessment of the P-ASK framework on rock art images . . . . . . . . 90

5.3.4 Model accuracy assessment of the image-based characterization . . . . . 92

5.3.5 Output sRGB characterized images . . . . . . . . . . . . . . . 93 
5.4 Discussion . . . . . . . . . . . . . . . . . 95

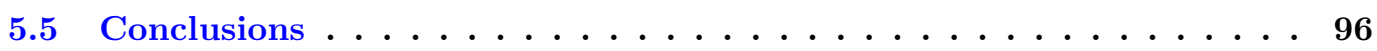




\section{$5.1 \quad$ Introduction}

As stated in Chapters 1 to 4, the camera characterization procedure has been recognized as a convenient methodology to correct color recordings in cultural heritage documentation and preservation tasks. The purpose of camera characterization is to transform the input device-dependent values captured by the camera, generally in RGB format, into a physically-based, independent color space, such as those defined by the CIE, usually the CIE XYZ color space or any of its derivatives (Westland et al., 2012). As a result of camera characterization, the tristimulus values for the full scene can be predicted on a pixel-by-pixel basis from the original digital values captured in the image (Molada-Tebar et al., 2018).

Some factors to consider during the characterization procedure are: the camera built-in sensor, camera parameters during the photographic acquisition phase, size and color properties of training data (Chou et al., 2013, Zhang et al., 2017), and the regression model used. The sensor has an effect not only in terms of noise but in the characterized output images (Molada-Tebar et al., 2019c). Thus, the interpolation method selected must be properly adapted to the data and offer a suitable graphic solution. The number of color patches measured and their distribution also plays a decisive role (Colantoni et al., 2011).

The manufactured color charts are designed to cover the maximum range of imaged colors on photographic or industrial applications. However, most applications do not require the entire set of color patches to build the training set since redundant data seep into the regression model. In addition, color charts are not developed specifically for archaeological purposes, where only a few colors are present on natural scenes. Thus, an adequate selection of training samples for the regression model is crucial for quality estimations (Cheung and Westland, 2006, Chou et al., 2013, Eckhard et al., 2014).

Our previous studies showed that accurate results can be achieved using a reduced number of properly selected samples. In Molada-Tebar et al. (2019a) we propose the use of skin-tone patches from the reference color checker as an alternative to using the whole color chart as training data. This paper clearly shows that an increase in the number of sample patches does not intrinsically imply an improvement in the goodness-of-fit of the output sRGB characterized image. In fact, better results are obtained using sample data near the chromatic range of the scene (skin-tone data set) with color differences values less than 4 CIELAB units. The results presented in that study revealed two key aspects in the characterization procedure, specifically the number of samples and the use of training data near the chromatic range of the scene used in the characterization procedure itself.

Accordingly and following the lines established in our previous investigations, in this chapter we propose a novel framework for an optimal color sample selection of training data. The algorithm is based on a $K$-means clustering technique. We call this procedure the Patch Adaptive Selection with $K$-means or P-ASK. The use of the P-ASK framework allows us to extract the dominant colors from a digital image and to identify their corresponding chips in the color chart used as colorimetric reference. 
The aim of the P-ASK dominant color patch selection is to carry out the characterization with a reduced color sample set, instead of using the common approach based on the whole color chart. In the P-ASK approach, it is possible to lessen the number of training samples without the loss of colorimetric quality, using the most representative samples within the chromatic range of the input scene. However, the main contribution reported in this chapter is not only the framework for the $K$-means methodology but the practical assessment of the results, specifically for recording and documenting the color of the rock art scenes properly.

Several methodologies can be found in the literature about optimal color sample selection, such as those based on metric formulas (Cheung and Westland, 2006), adaptive training set (Colantoni et al., 2011, Liu et al., 2017), or minimizing the root-mean-square errors (RMSE) (Shen et al., 2008). Olejnik-Krugly and Korytkowski (2020) developed a procedure based on ICC profiles using a standard ColorChecker and a set of custom color targets by means of direct measurements on the artwork .

Clustering analysis is widely applied for the extraction of representative samples (Mohammadi et al., 2004, Xu et al., 2017, Liu et al., 2017). Eckhard et al. (2014) proposed a clustering-based novel adaptive global training set selection and compared different well established global methods for colorimetric quality assessment of printed inks. Xu et al. (2017) applied a $K$-means clustering algorithm in multispectral images to extract a set of training samples directly from the art painting for spectral image reconstruction applications. These studies showed that better results were achieved using optimal color samples.

We tested the P-ASK framework proposed in this chapter on a set of prehistoric rock art scenes captured with different digital cameras. The characterization approach with dominant colors offered numerous advantages: reduction of training measurement and computing time, better adjustment to the chromatic range of the input scene, and efficient and high model accuracy achievement. But most importantly, the numeric and graphic results obtained in the characterized images were very satisfactory and confirmed the P-ASK technique as a suitable methodology for model training in characterization procedures.

\subsection{Materials and Methods}

\subsubsection{Data set acquisition}

The image set contains the so-called main hunting scene ("Cova dels Cavalls", Figure 1.1), which shows a group of hunters shooting arrows with their bows to a herd of deer (Figure 5.1). It is undoubtedly a vivid and spectacular human-centered scene, filled with movement, where the use of reddish pigment dominates the scene (Martínez and Villaverde, 2002).

Three different SLR cameras were used in this study for image acquisition: a Fujifilm IS PRO with a Super CCD sensor; a Sigma SD15 with a CMOS Foveon $囚 X 3$ sensor; and a Nikon D40, with a CCD sensor (Table 1.1). All those cameras allow taking pictures in a RAW format, which gives better results than processed images after characterization (Westland et al., 2012, Molada-Tebar 
et al., 2018). In fact, the use of RAW images allows us to disable the activation of the set of processing operations applied to the original images, such as white balancing, demosaicing or gamma correction, which implies the modification of the original data acquired by the sensor (Ramanath et al., 2005, Westland et al., 2012).

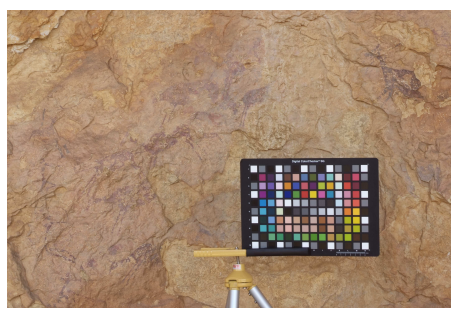

(a)

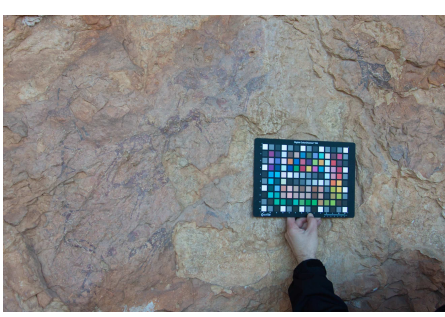

(b)

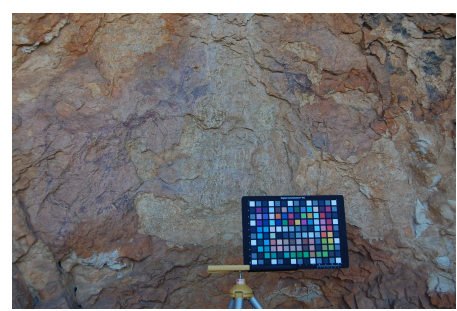

(c)

Figure 5.1: Rock art images: (a) Fujifilm IS PRO (b) Sigma SD15 (c) Nikon D40.

The main difference across cameras is their built-in image sensors. While the Super CCD sensor (Fujifilm IS PRO) and CCD sensor (Nikon D40) are monochrome with color filter array (CFA) and Bayern pattern for color interpolation, the Foveon $\AA \mathrm{X} 3$ sensor (Sigma SD15) is trichromatic, that is, it registers the color values for each channel separately without interpolation (Sigma Corporation, 2012).

As in the previous experiments, the scenes in the digital images should contain a chart for

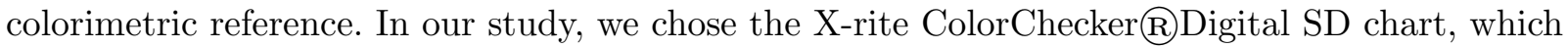
is widely used in digital image processing pipelines. Although this color chart has 140 patches, we used only the 96 chromatic central chips (and 15 grey-scale central chips) without considering the external patches on the edges. The RAW RGB data for these patches were extracted from the images using our own software named pyColourimetry (Molada-Tebar et al., 2017).

The spectral reflectance data of the color patches from the ColorChecker were measured directly using the Konica Minolta CM-600d spectrophotometer (Figure $2.1 \mathrm{c}$ ). A total of 96 patches were measured under the CIE recommendations: $2^{\circ}$ standard observer, and D65 illuminant. The spectral data collected were easily transformed into CIE XYZ tristimulus values using well-known CIE formulae (CIE, 2018).

\subsubsection{Colorimetric camera characterization procedure}

As introduced in Chapter 1 to 3, image-based camera characterization is a well-known procedure that has been described in numerous references (Hong et al., 2001, Martínez-Verdú et al., 2003, Bianco et al., 2009, Westland et al., 2012, Molada-Tebar et al., 2018). The main stages of the image-based camera characterization procedure are (1) Training: sets the training data for model learning, (2) Regression model fitting and checking, and (3) Image characterization: creates the output sRGB image (Figure 5.2). In the following subsections, we provide detailed descriptions of each step. 


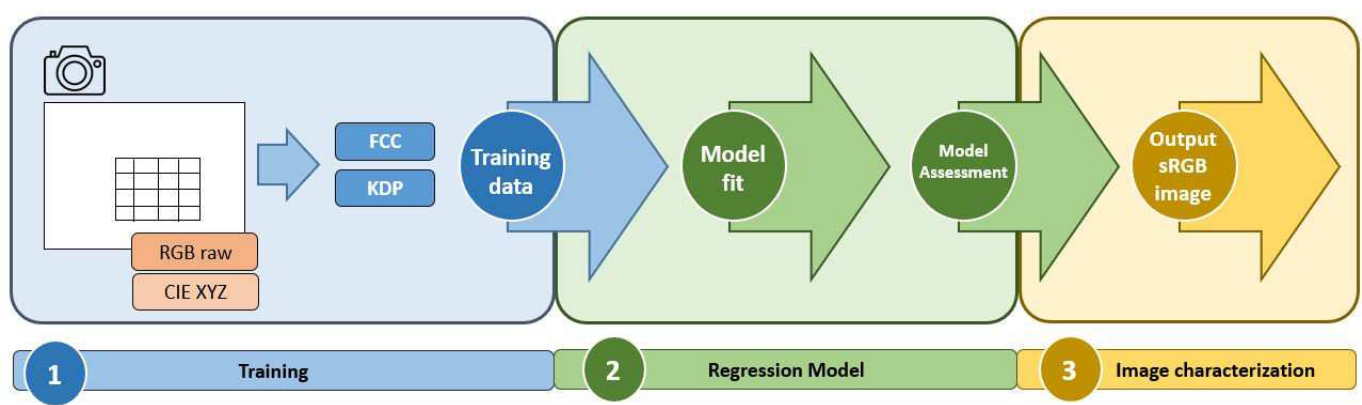

Figure 5.2: Colorimetric camera characterization framework.

\subsubsection{Training data}

Image-based characterization procedures rely on the training data set that typically permits model learning via the computation of the unknown parameters in the selected mathematical function. The regression model is developed on the basis of the original data available: the camera responses (RAW RGB) and their colorimetric coordinates (CIE XYZ), both from the reference color chart.

As for the comparative analysis, we created two subsets of training data from each original dataset: the whole color patch set (Full ColorChecker or FCC data set), and the selected dominant patches from the P-ASK framework ( $K$-means dominant patches or KDP data set). Therefore, we obtained two independent outcomes as outlined in Figure 5.2.

\subsubsection{Regression model and accuracy assessment}

As usual, the image-based characterization procedure used in this study is based on an empirical regression model that defines the relationship between the input and output spaces. Different regression models have been used for camera characterization purposes, including polynomial models (Hong et al., 2001), artificial neural networks (Cheung and Westland, 2002), polyharmonic splines (Colantoni et al., 2011) or Gaussian processes (Molada-Tebar et al., 2019c) to name a few. However, in recent years, research has focused on spectral recovery techniques (Liang and Wan, 2017). In this study, we used a classical second-order polynomial regression model, since it is computationally simple and gives valid results for archaeological applications (Molada-Tebar et al., 2018, 2019c).

In terms of good practice, the training data used for learning should not be used in model assessment since it may result in an overly optimistic model error estimation. Instead, it is preferred to set aside an independent test data set used exclusively for model evaluation. Alternatively, a cross-validation framework can also be used, where the training data is repeatedly separated into training and testing sub data sets. In this study, a leave-one-out cross validation (LOOCV) procedure was performed for model accuracy assessment (Stone, 1974, Cawley and Talbot, 2003). 
Two different metrics were used for the quality evaluation: the CIE XYZ residuals for model checking, and the color difference values in the colorimetric assessment of the characterized images. Regarding the color difference computation we used the $\Delta E_{a b}^{*}$ formula (Eq. 3.3, (CIE, 2018)) which performs well in rock art measurement conditions (Molada-Tebar et al., 2019c). In colorimetric accuracy studies, it is usual to establish a threshold value known as JND or tolerance for $\Delta E_{a b}^{*}$. In this study we set a JND value of 4 CIELAB units which ensures imperceptible variations to human vision (Van Dormolen, 2012).

\subsubsection{Output sRGB characterized image}

An additional transformation is required in order to reproduce the output CIE XYZ values on display or printing devices. We followed the International Electrotechnical Commission (IEC) specifications to transform the CIE XYZ tristimulus values into the sRGB color space, which is compatible with most digital devices (IEC, 1998).

\subsection{3 $K$-means clustering technique}

$K$-means is a center-based clustering algorithm widely used in different applications of data mining, machine learning and pattern recognition (MacQueen, 1967). $K$-means aims to minimize the mean squared distance from each $N$-data point to its $K$-nearest cluster center, following four basic steps to: (1) set initial $K$ cluster centers, (2) group samples in the $K$ clusters, (3) recompute the new $K$ cluster centers, (4) iterate the process until the algorithm converges (Peña et al., 1999, Hamerly and Elkan, 2002, Joydeep and Alexander , 2009).

Since the introduction of the $K$-means algorithm in its current form (MacQueen, 1967), different alternatives and improvements to achieve better performance have been published, including an algorithm less sensitive to initialization settings (Hamerly and Elkan, 2002) or filtering algorithms (Kanungo et al., 2002).

In this study, we developed a Python script to apply the $K$-means algorithm to our specific problem, which we recall, is to select dominant colors in camera characterization. The Python script leverages the Scikit-learn package (v0.21.3) (Scikit-learn, 2019) which includes an optimized implementation of the $K$-means algorithm together with other machine learning algorithms for supervised and unsupervised classification applications (Pedregosa et al., 2011, Scikit-learn, 2019). Particularly, we used the KMeans function (see KMeans (2019) for more details).

However, before applying the $K$-means clustering algorithm, it is required to take into account some quirks associated with this technique. $K$-means yields accurate results when the $K$ number of clusters and its initial centroid points are properly defined. Moreover, as an iterative technique, the $K$-means algorithm is potentially sensitive to initial starting conditions (Bradley and Fayyad, 1998). Thus, an inappropriate determination of initial parameters could highly decrease the accuracy of the results (Limwattanapibool and Arch-int, 2017).

In our study, the inappropriate selection of clusters means that the results will not match with the real dominant colors present in the image. Therefore, it is necessary to overcome the 
negative effects derived from $K$-means initial approximations by properly setting the $K$ number of clusters for optimal implementation of the algorithm and proper determination of the initial values.

\subsubsection{Optimal $K$ number of clusters}

The first issue to address is how to set the optimal $K$ number of clusters and their corresponding initial centroids, since the $K$ clusters should be known beforehand and specified by the user. Several factors such as the level of detail, the internal distribution of the samples values or its interdependence with other objects can affect the selection of $K$ (Peña et al., 1999, Pham et al., 2005). To overcome those issues, different methods can be applied for selecting the number of clusters, such as those based on automatic process (Limwattanapibool and Arch-int, 2017), probabilistic clustering approaches or visual verification (Pham et al., 2005).

To determine an appropriate number of clusters for $K$-means clustering, a trial-and-error process experiment was conducted. We used the rock art scene images as input data to evaluate the processing time for different values of $K$. In general, $K$-means clustering is used not only to determine the natural groups in the data, but also to identify irregularities in their distribution. However, in rock art scenes the data tends to be uniform, and we expect a set of dominant colors consisting of a reduced number of instances, which in turn will be highly correlated. In order to determine the proper value of $K$, we assessed the processing time for values of $K$ in the range $10-100$.

\subsubsection{Clustering Algorithm}

The basic $K$-means algorithm (MacQueen, 1967) has been updated to improve its effectiveness, robustness and convergence speed (Hamerly and Elkan, 2002). Some of the most widely known implementations are: Forgi (Forgi, 1965), expectation-maximization or EM-Style algorithm (Demster et al., 1977, Bottou et al., 1995), Standard algorithm also known as LLoyd's algorithm or Voroni iteration (Lloyd, 1982), and Elkan (Elkan, 2003).

Two of those algorithms are implemented in the Scikit-learn KMeans function: EM-style for sparse data and Elkan for dense data. Users can specify their choice via the algorithm parameter, using full or Elkan for the EM-style, or Elkan option respectively (see an example of code in Section 5.2.4.1 for syntax details). Given that our study is based on moderately large data sets from the RGB triplets of the digital image, the optimal algorithm to compute $K$-means is Elkan. The Elkan algorithm is an optimized version of the standard $K$-means clustering method based on the triangle inequality. The aim is to reduce its processing time by avoiding unnecessary distance calculations (Elkan, 2003). 


\subsubsection{Initial starting conditions}

The $K$-means algorithm searches for the optimal data partition into the $K$ clusters established by the user. Thus, $K$-means provides a set of $K$ point clusters defined by their centroids, and assigns every data point into its nearest cluster centroid. However, the final group partition is highly dependent on the initial cluster centers. Since the centroids of the clusters are usually unknown in advance, the $K$-means algorithm relies on a random process to set the starting cluster centroids, which are frequently chosen uniformly on the range of the data (Bradley and Fayyad, 1998).

The random initialization method takes a set of $K$ arbitrary centers uniformly selected at random as starting points. The drawback of purely random selection is the high number of iterations required to converge, and furthermore, different runs with the same data do not give exactly the same results. In order to overcome this limitation, the $k$-means ++ initial method was developed to improve algorithm speed and give more consistent results despite being a random method (Arthur and Vassilvitskii, 2007). In fact, this is the default option for the KMeans function.

The lack of consistency of random methods has been the subject of previous research. However, initialization methods based on randomness are considered as a proper selection procedure that gives satisfactory results (Peña et al., 1999). In this study, we run a simple experiment to find out the optimal initial values based on random algorithms. We run the algorithm several times which has been reported as a suitable method to account for randomness issues (Bradley and Fayyad, 1998). We used the Scikit-learn $k$-means ++ initial method, since it is an optimized and fast random process (Arthur and Vassilvitskii, 2007). This analysis will allow us to determine whether or not the algorithm consistently provides the same dominant color patches despite being based on a random process.

\subsubsection{Color patch adaptive selection with $K$-means (P-ASK)}

The aim of the P-ASK procedure is to establish a methodology for the automatic selection of color training samples for camera characterization, and keep the process as simple as possible. The P-ASK framework can be separated into two main stages: the application of the $K$-means algorithm for the dominant color extraction of the input image, and the identification of dominant color patches as training data for the camera characterization. The first stage of this procedure can be considered as an image segmentation application, which is intended to group the pixels of an image according to their color similarity.

The main steps in the P-ASK framework (Figure 5.3) are: (1) create a white balanced image (D65 illuminant), (2) select support and pigment samples, (3) extract $K$-means dominant colors, and (4) identify dominant color patches. Further detailed explanations about those steps are given in the subsections below. 


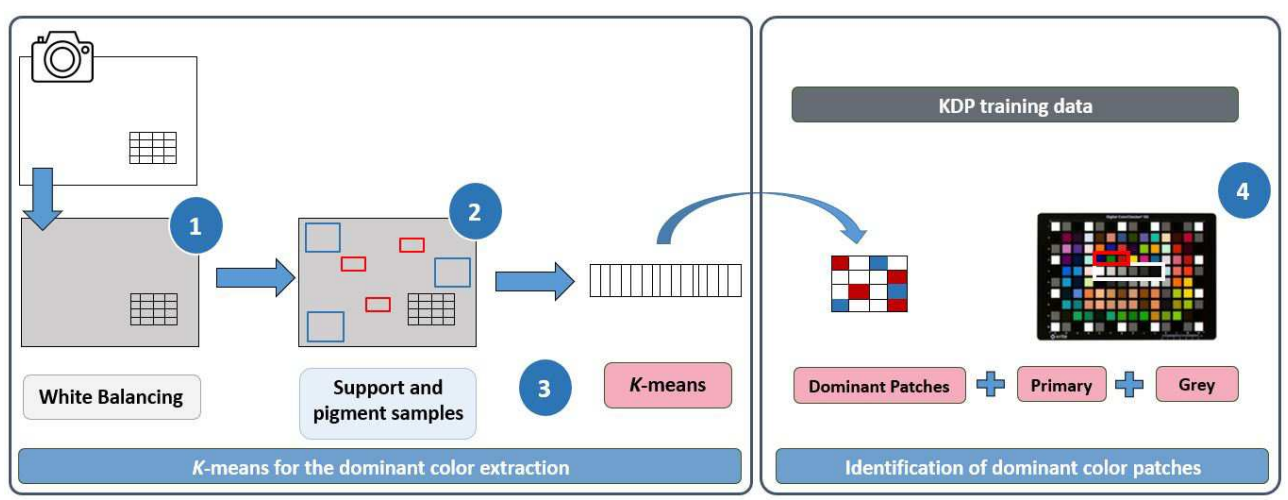

Figure 5.3: P-ASK framework.

\subsubsection{1 $K$-means dominant color extraction}

The first aim of P-ASK is the dominant color extraction from a digital image. Previously to applying the $K$-means clustering algorithm two basic operations are required: white balance and sample selection.

The CIE XYZ data were acquired under the D65 illuminant specification. Thus, in order to find the patches corresponding to dominant colors, the RGB values should be strictly registered under the D65 illuminant. Since it is not usually possible to work under controlled illuminant conditions in open-air cultural heritage sites such as rock art archaeological shelters, we used a white balanced image under the hypothesis of D65 illuminant as an approximation to natural daylight. From an original RAW image, it is possible to obtain a white balanced image using the RawPy Python package (RawPy, 2014).

One problem associated with the application of the $K$-means clustering algorithm to full images is the significant increase of the convergence time, given the large size of the data set. A simple and suitable option in our framework is to reduce the image size to compute only selected image samples instead of full images. For rock art applications, the main interest is focused on defining the representative colors, especially of the pigments and the main support colors. Thus, we selected a set of samples from significant support and pigment areas for the $K$-means computation (Figure 5.4).

Finally, the digital data are passed into the algorithm as RGB triplets (nRGBdarray) for subsequent computing. The Python code line which runs the $K$-means algorithm is as follows:

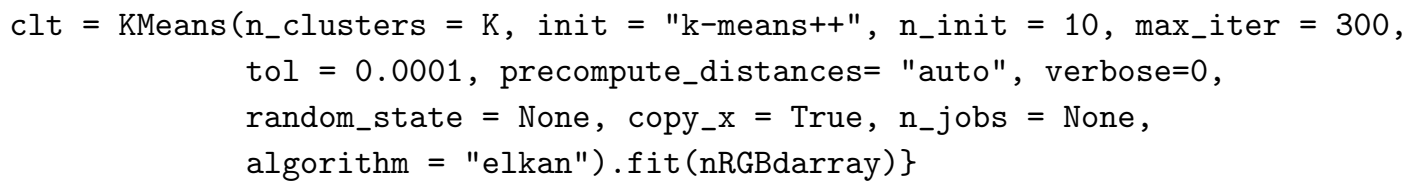

where the main parameters introduced to the algorithm are: the $K$ number of clusters; Elkan as the algorithm since it works well for dense data; and the $k$-means ++ initialization method. 
The remaining parameters have been assigned to the default values of the Scikit-learn KMeans function (KMeans, 2019).

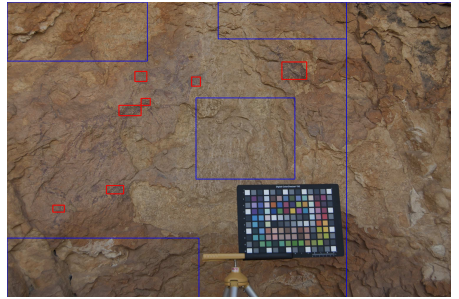

(a)

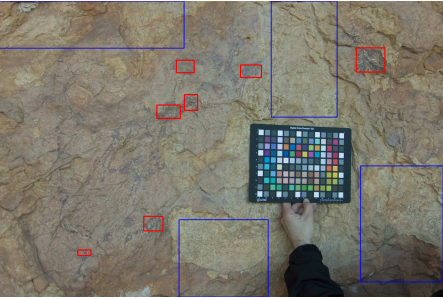

(b)

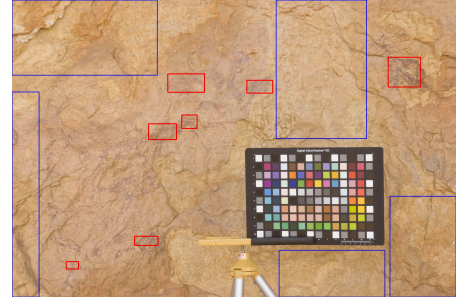

(c)

Figure 5.4: Support and pigment samples: (a) Nikon D40 image (b) Sigma SD15 image (c) Fujifilm IS PRO image.

\subsubsection{Identification of dominant color patches}

The outcome of the $K$-means algorithm consists of a set of centroids, one for each $K$ cluster. Those coordinates are a list of $K$ RGB triplets of dominant colors, which represent the main colors found in the input digital image. The second stage seeks the correct identification of the color chart patches from the dominant colors from the $K$-means algorithm.

The dominant colors extracted in the RGB color space can be easily transformed into CIE XYZ tristimulus values (CIE, 2018) and CIELAB coordinates (CIE, 2007) as required for subsequent processing. With the CIELAB coordinates, the color differences are computed between each dominant color and the whole set of color chips. It is then possible to identify the nearest patches to each dominant color found in the image by looking for the lowest $\Delta E_{a b}^{*}$.

For the identification of color patches, four different approaches are tested after varying the input data and colorimetric criteria: (1) using the $\mathrm{n}$ nearest patches found in the whole image $(\mathrm{NN}),(2)$ removing the color chart from the scene and identifying the n nearest patches (RC), (3) using a set of samples corresponding to pigment and support classes and creating a new, joined synthetic image (JS), and (4) using the same sample set used in (3), but computing each class separately (SS). Note that while in the first method we do not specify any colorimetric criteria, in the remaining cases we search for the nearest patches to each dominant color with $\Delta E_{a b}^{*}$ color differences less than 10 CIELAB units (upper bound value allowed in cultural heritage guidelines (Van Dormolen, 2012)).

Although the use of the nearest patches extracted from dominant colors using $K$-means offer accurate colorimetric results, it does not always imply a proper output characterized image, that is, an image with true colorimetric sense. Our experience shows that a set of minimum fixed patches should be included in order to obtain accurate and colorimetrically sound characterized images (Molada-Tebar et al., 2019a), including the primary additive colors [E4, F4, G4] and the basic gray-scale chips [E5, F5, G5, H5, I5, J5] (highlighted in Figure 5.3, step number 4). 


\subsection{Results}

In this section, we describe all processing tasks, together with their output results, that support some practical considerations to define the P-ASK. Specific tests were run to define the optimal parameters of the algorithm and to assess its performance in controlled laboratory conditions as well as in rock art sites with uncontrolled light conditions.

\subsubsection{Setting the optimal parameters for the $K$-means clustering algorithm}

As reported in Section 5.2.3, several factors could affect the results of the $K$-means clustering method, namely the $K$ number of clusters and centroids to generate, the input size image, the initialization method and the algorithm applied. We discuss each individual factor in this section.

\subsubsection{1 $K$ number of clusters}

The first issue to consider is the proper determination of the $K$ number of clusters. That number can be determined by simple heuristics, for instance by testing several values of $K$ and taking some convenient value, or with specific data mining tools such as the Elbow (Thorndike, 1953, Bholowalia et al., 2014) or the Silhouette graphic methods (Rousseeuw, 1987).

A preliminary experiment was conducted under the worst-case scenario; that is, using the whole image as input data which gives some sort of upper bound in terms of computation time. The results of the running time required for computing $K$-means clustering are displayed in Table 5.1. According to those results, $K=24$ seems to be an acceptable number of clusters. The choice of this value, however, is based on the running time experiment only, and thereby it is somewhat arbitrary. It is timely, then, to confirm our choice by other means, with a consensus across numerical results, graphical tools and colorimetric criteria.

Table 5.1: Evaluation of $K$-means running time with different values of $K$

\begin{tabular}{ccccc}
\hline K & $\begin{array}{c}\text { Nikon D40 } \\
\text { Time (min) }\end{array}$ & $\begin{array}{c}\text { Sigma SD15 } \\
\text { Time (min) }\end{array}$ & $\begin{array}{c}\text { Fujifilm IS PRO } \\
\text { Time (min) }\end{array}$ & $\begin{array}{c}\text { Mean } \\
\text { Time (min) }\end{array}$ \\
\hline 10 & 8.0 & 5.2 & 7.6 & 6.9 \\
20 & 17.1 & 16.7 & 15.9 & 16.6 \\
24 & 19.3 & 18.4 & 16.9 & 18.2 \\
30 & 27.4 & 25.5 & 24.2 & 25.7 \\
40 & 31.8 & 30.8 & 30.6 & 31.1 \\
50 & 45.1 & 37.9 & 38.1 & 40.4 \\
60 & 49.5 & 49.2 & 53.5 & 50.7 \\
80 & 67.8 & 60.2 & 69.1 & 65.7 \\
100 & 87.9 & 76.0 & 100.3 & 88.1 \\
\hline
\end{tabular}

From a color viewpoint, our study aims at characterizing digital cameras properly with a reduced number of color chips whether it is possible, which is in many ways rather the same 
as performing color quantization of the image. In addition, our application field, the recording of Levantine rock art archaeological shelters requires a very specific color domain, mostly in the chromatic range of red, brown, and yellow colors which in turn are little saturated (check dominant colors extracted in rock art images in Section 5.3.3). Thus, a number of 24 seems a reasonable choice, provided that output images are rigorous duplicates of the real specimens (Section 5.3.4 and Section 5.3.5 report on this point).

In addition to colorimetric criteria, we tested the Elbow and Silhouette methods which provide graphic aid to determine the right $K$ number in clustering problems. The Elbow method (Thorndike, 1953, Bholowalia et al., 2014) consists of running the $K$-means algorithm on the dataset for a range of values of $K$, and calculate the distortion and inertia values for each $K$. The distortion value is the average of the Euclidean distance between all the cluster centroids for each $K$; the inertia is calculated as the sum of the Euclidean distances to their nearest cluster centroids. Those parameters are useful to describe how close the different $K$ clusters are with respect to each other. Clusters that are too close may be an indication of incorrect data partitioning, so that they could be integrated into the same cluster.

Figure 5.5 shows the plot of the Elbow analysis using the inertia criterion, which exhibits a sharp change in slope around $K=24$ (cf. vertical red line on Figure 5.5) and linear behavior beyond this $K$ value. This result is compatible with the previous running time test (Table 5.1).

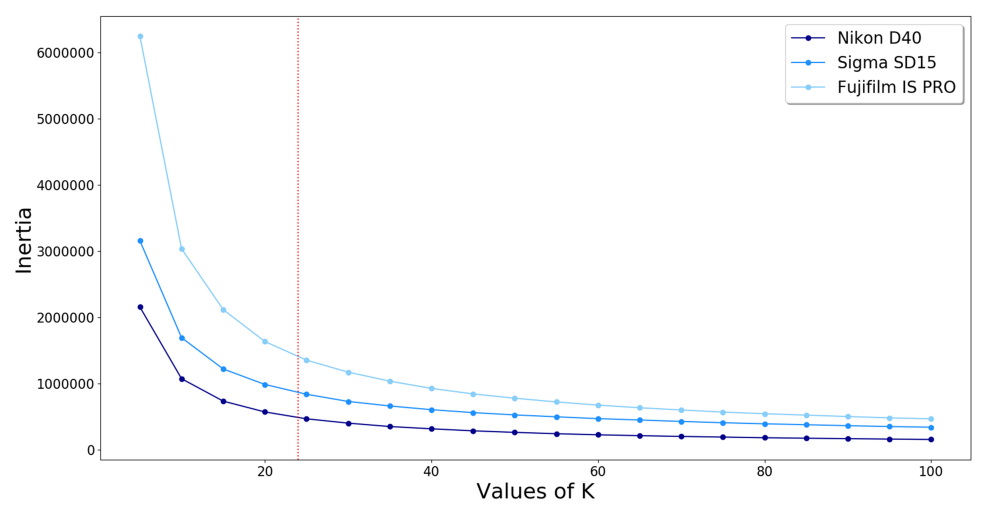

Figure 5.5: Elbow method using inertia

The silhouette analysis is another useful tool to analyze the separation distance among cluster centroids, which ultimately reports on the quality of the data partition. In this method, each $K$ cluster is represented by its so-called silhouette, which is calculated as a result of the comparison between its tightness and separation (Rousseeuw, 1987). The mean silhouette width, also known as the silhouette coefficient or silhouette score, provides an evaluation of clustering validity, with a value within the range $[-1,1]$. Negative values indicate wrong cluster assignment, whereas positive values (the closer to 1 , the better) indicate well-separated neighboring clusters. 
We run the Silhouette analysis using the Scikit-learn module which implements the method in two functions called metrics.silhouette_score and metrics.silhouette_samples. Both functions require the output of the KMeans function as input parameter (Silhouette, 2019). Figure 5.6 displays the graphic results given by the Silhouette method for the Fujifilm IS PRO image with $K=24$. All silhouette coefficients are similar (around 0.6) with narrow fluctuations, which is an indicator of good clustering for the chosen number of clusters K (Burney and Tariq, 2014). Furthermore, all coefficients are above the average silhouette score (vertical red dashed line in Figure 5.6 a) which confirms the value 24 as a proper choice for clustering. The silhouette method also provides a graph for visualizing the clustered data (Figure $5.6 \mathrm{~b}$ ), which shows a very dense, but well separated, data set as expected in our case study.

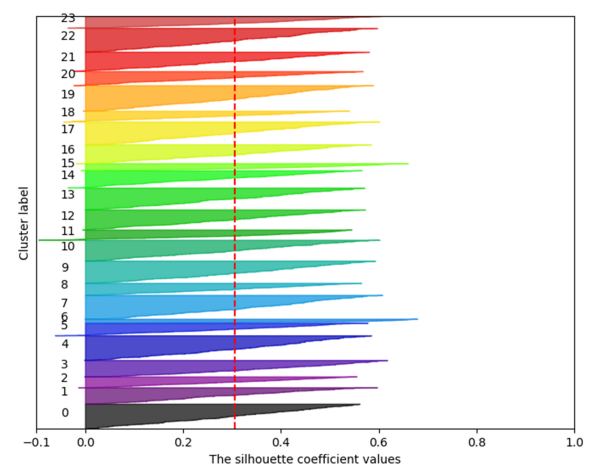

(a)

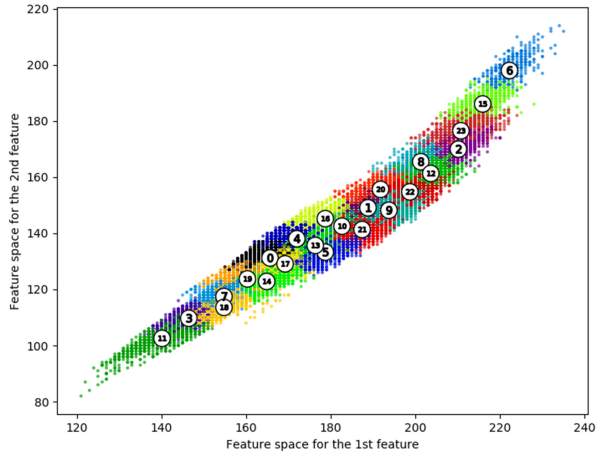

(b)

Figure 5.6: Silhouette analysis for the Fujifilm IS PRO

\subsubsection{K-means initialization}

Once the $K$ number of clusters is determined, the next step is the proper selection of the algorithm and the initialization method to run $K$-means. The KMeans function in the Scikit-learn Python package provides two algorithms: EM-style and Elkan. Since we usually work with large, dense data sets collected from digital images, we chose the Elkan algorithm which is known to perform well in such circumstances (Elkan, 2003, KMeans, 2019).

The second parameter to be determined is the initialization method of the algorithm. As usual in all implementations, the KMeans function relies on a pseudo-random parameter to start the process. Theoretically, the best option in the KMeans function is the $k$-means ++ method, which is an optimized and fast, but still random approach (KMeans, 2019). Random initialization is a convenient approach to create self-starting methods and reduce user intervention. However, it has the potential drawback of returning different or inconsistent results in different runs (Pham et al., 2005, Limwattanapibool and Arch-int, 2017). This characteristic is clearly against common scientific and engineering conventions such as repeatability or reproducibility, and its effects on the results must be verified. 
In our study, the $K$-means algorithm was run multiple times (100 realizations) to find out whether or not the $K$-means reported the same set of dominant colors consistently. We then grouped the results by dominant color and created a list of RGB triplets for each dominant color found. Every list was processed to compute basic statistics: mean, minimum, maximum, standard deviation, coefficient of variation, and so on. Those results confirmed that the $K$-means algorithm reports dominant colors very consistently. The RGB differences within every dominant color were all negligible which also applies when we computed the $\Delta E_{a b}^{*}$ differences of the data (Figure 5.7). All color difference values were less than 1 CIELAB unit $\left(\Delta E_{a b}^{*}<1\right.$, red line on Figure 5.7 a), clearly under the JND threshold value, and the standard deviation error was less than 3 digital level units (Figure $5.7 \mathrm{~b}$ ). Furthermore, and most importantly, the set of colors is consistently in the same range, which confirms the suitability of the $K$-means algorithm to determine dominant color in rock art scenes for camera characterization purposes.

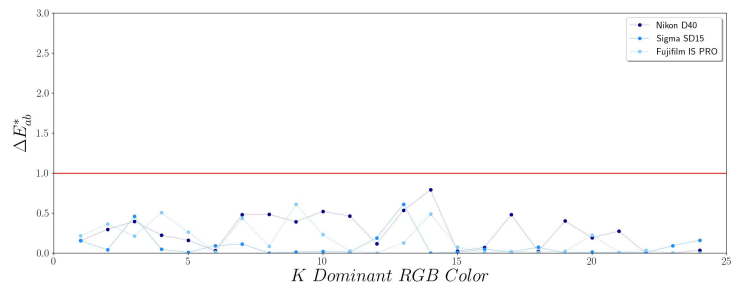

(a)

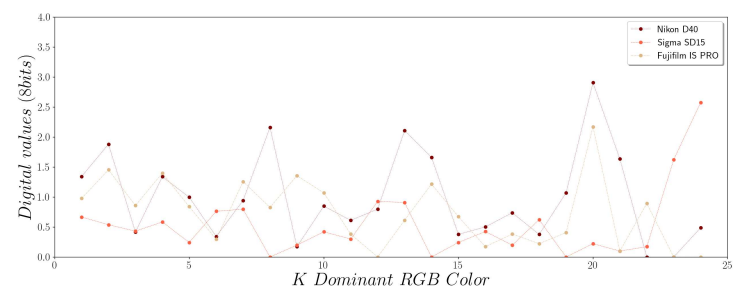

(b)

Figure 5.7: Randomness assessment: (a) $\Delta E_{a b}^{*}$ color differences (b) Standard deviation error

\subsubsection{Identification of dominant color patches}

The input image size, that is the data set size, has a direct effect on the processing time required for the $K$-means algorithm (Section 5.3.1.1). A simple solution to reduce the processing time is to use small sample images instead of the complete picture. However, our study seeks an efficient methodology not only in terms of computation speed, but mostly regarding its performance in identifying color patches. In this line, four different methods were tested prior to setting the optimal path for identifying color patches. Table 5.2 shows the results of each method. Those four methods are briefly described as follows:

- Nearest n-patches (NN). The complete image is processed.

- Remove ColorChart (RC). The color chart is removed from the original image.

- Joined samples (JS). Pigment and support samples are merged in a new synthetic data set and processed together.

- Split samples (SS). Pigment and support samples are processed using two different data sets. 
Table 5.2: Comparison of four methods to identify dominant color patches

\begin{tabular}{lcccc}
\hline Camera & NN & RC & JS & SS \\
\hline Nikon D40 & 18 & 5 & 13 & 17 \\
Sigma SD15 & 21 & 12 & 13 & 16 \\
Fujifilm IS PRO & 15 & 12 & 13 & 15 \\
Average & 18 & 10 & 13 & 16 \\
\hline
\end{tabular}

The NN method detected the highest number of patches using the four nearest patches $(n=4)$. It should be noted, though, that there are no colorimetric restrictions in this approach, which means that some patches can be out of the range of dominant colors extracted from the images or, in other words, some patches may not fit the real rock art scene. This point came out when observing some visual inconsistencies between the patches detected by the algorithm and the scenes under study. The other methods detected fewer patches but returned better colorimetric results. This was the expected result since colorimetric constraints somehow guarantee a better fit of the selected patches to the scene. The RC method systematically gave the smallest number of patches and should be rejected since the other three methods provide similar results regardless of the camera sensor in use. The method that offered the best results was the SS, therefore, splitting samples for the pigment and the support areas seems to be the most reasonable choice.

We also conducted a detailed analysis of the SS method with a focus on performance as a function of the number of clusters $K$ (Table 5.3 and Figure 5.8). According to these results, the number of color patches detected stabilizes, whereas the processing time grows linearly without improving the collection of color patches. Therefore, based on these results the best option seems to be the SS method with $K=24$.

Table 5.3: Assessment of the SS method for the identification of dominant patches

\begin{tabular}{ccccccc}
\hline & \multicolumn{2}{c}{ Nikon D40 } & \multicolumn{2}{c}{ Sigma SD15 } & \multicolumn{2}{c}{ Fujifilm IS PRO } \\
K & Patches & Time & Patches & Time & Patches & Time \\
\hline 10 & 14 & 3.6 & 15 & 1.7 & 15 & 2.0 \\
20 & 15 & 8.0 & 16 & 3.3 & 15 & 4.3 \\
24 & 17 & 9.7 & 16 & 4.0 & 15 & 4.9 \\
30 & 17 & 9.3 & 16 & 5.0 & 15 & 5.8 \\
40 & 18 & 12.8 & 17 & 6.0 & 16 & 6.5 \\
50 & 19 & 14.9 & 17 & 7.2 & 17 & 9.0 \\
60 & 20 & 16.8 & 17 & 8.7 & 17 & 10.5 \\
80 & 21 & 23.1 & 17 & 11.6 & 17 & 13.4 \\
100 & 21 & 26.3 & 17 & 13.2 & 17 & 16.0 \\
\hline
\end{tabular}




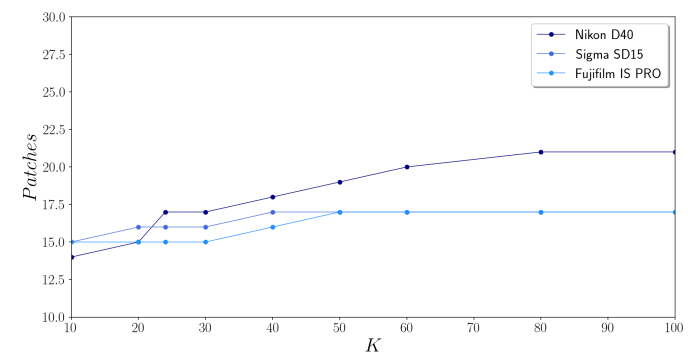

(a)

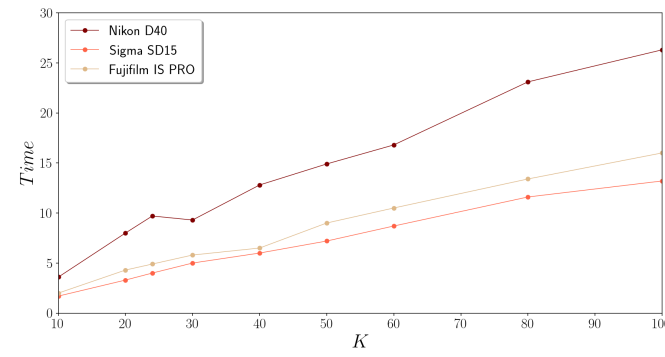

(b)

Figure 5.8: Graphic results for the SS method: (a) Patches identified; (b) Processing time.

\subsubsection{Test on the DigiEye imaging system}

Before applying the P-ASK framework on rock art images, several tests were run in a laboratory environment under highly controlled light conditions. We used the DigiEye imaging system (Verivide, 2019) which allows taking pictures in an optimal environment to conduct colorimetry experiments. The images were captured with the SLR Nikon D40 camera and the color reference was the X-rite Passport color chart under the D65 illuminant (Figure 5.9).

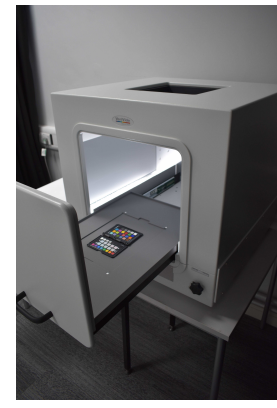

(a)

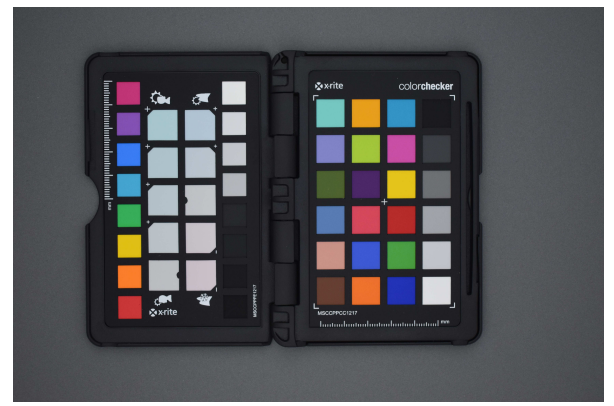

(b)

Figure 5.9: DigiEye imaging system: (a) DigiEye system (without camera integrated) (b) Nikon D40 test image (D65 illuminant).

The test presented in this Section is a reduced version of the whole P-ASK method. In the test we obtained images of the color checker, without rock art specimens, which provide some sort of "benchmark environment" to assess the quality of P-ASK (Figure 5.10).

The input image was a clipped picture of the X-rite color checker and the working parameters were: $K=25$ (24 colored patches and one color for the background), Elkan algorithm, $k$-means ++ initialization, and the SS method for the identification of the dominant patches. Figure 5.10 shows the performance in the identification of colors on the chart. Our method can perfectly identify all colors present in the chart without any missing patches. This result is certainly compelling and shows the potential of P-ASK to adapt to the whole chromatic domain of the scene, which makes the method a suitable choice to be applied in documenting rock art images. 


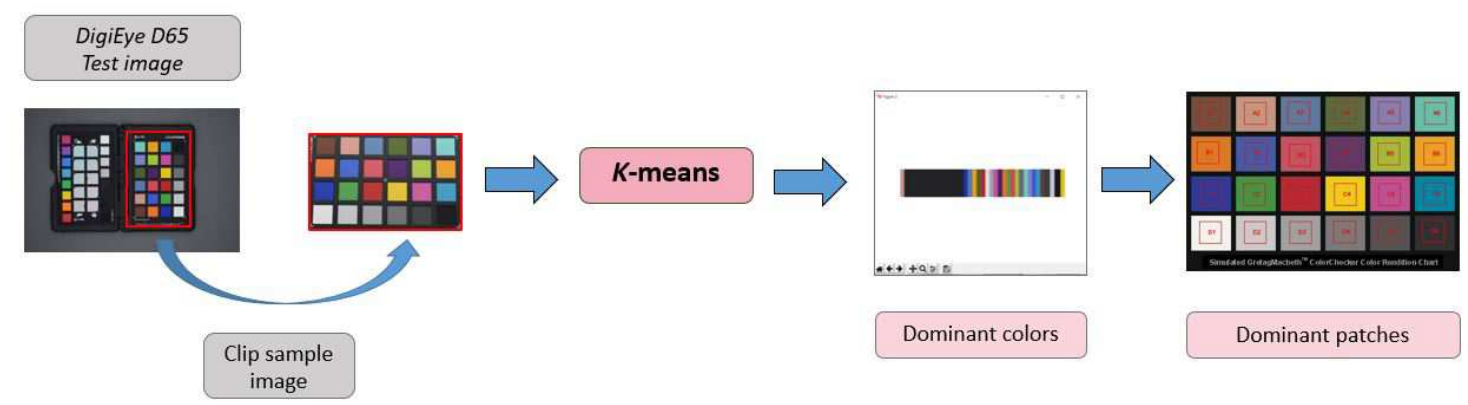

Figure 5.10: P-ASK framework adapted to the DigiEye D65 image acquired with the Nikon D40.

\subsubsection{Assessment of the P-ASK framework on rock art images}

Since the P-ASK framework provides good results in laboratory conditions, the next step is the assessment using images collected in rock art shelters. In order to simplify this chapter, we present the results for the Fujifilm IS PRO image only. We should note that all steps described in Figure 5.3 were carefully computed, using the parameters established in previous sections: $K=24$, Elkan algorithm, $k$-means ++ initialization, and SS method. We emphasize once again that the aim of the P-ASK framework is to (a) extract the dominant colors present on the rock art scene, (b) identify its corresponding patches in the color chart, and (c) carry out the characterization using those patches as training data.

From the Fujifilm IS PRO white-balanced image, a set of twelve samples were collected for the extraction of dominant colors, five samples for the support (S1, S2, S3, S4 and S5), and seven for the pigment (P1, P2, P3, P4, P5, P6 and P7). Figure $5.4 \mathrm{c}$ shows the sampling areas that were clipped to get the samples for subsequent processing. Figure 5.11 contains a detailed description of pigment sample $\mathrm{P} 1$ consisting of the detail clipped image, the color palette of the dominant colors, and its frequency histogram. P-ASK provides this information for each sample separately.

The last step prior to camera characterization is to select a list of patches from the color chart that matches with the dominant colors obtained from the samples. The results provided by P-ASK consist of 9 patches for the support and 14 for the pigment (see a summary in Table 5.4 and Figure 5.12). This is the expected result considering that the support rock material is highly uniform and requires fewer colors to be characterized. Note also that duplicates appear across samples of the same class (either pigment or support) as well as across samples of different classes. This characteristic is also expected since the sampling areas for pigment characterization also contain some amount of support.

A total of 15 color patches are identified from the chromatic image information with P-ASK (Table 5.4 and Figure $5.12 \mathrm{c}$ ). We have to note that none of the previous methods integrate "color insight" in their rationales. It is the user's responsibility to add such insight, as we did when extending the set of color chips with three primaries and six grays. Thus, the KDP training dataset for the Fujifilm IS PRO camera consists of a total of 24 color patches (cf. Figure 5.12 d). 


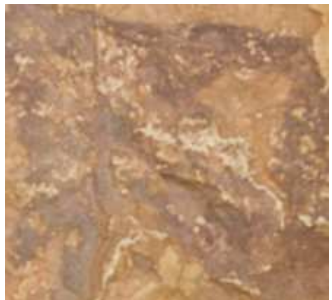

(a)

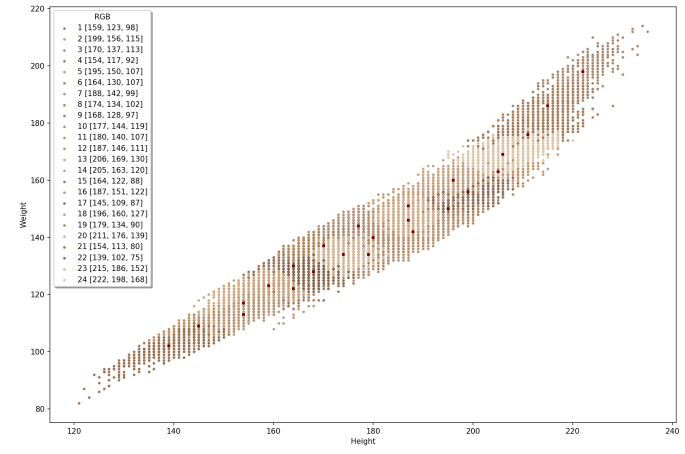

(b)

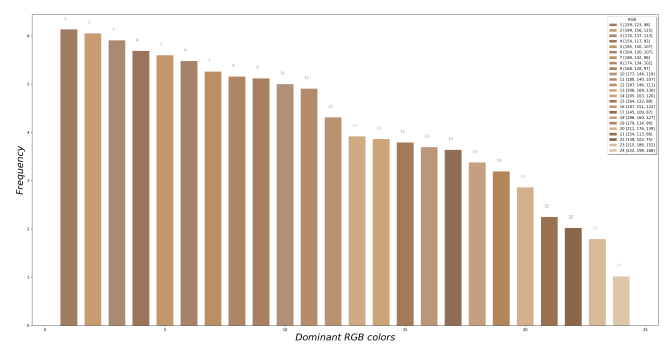

(d)

Figure 5.11: Graphic description of the pigment sample (P1): (a) clipped detail image, (b) $K$-means centroids, (c) Dominant color palette, (d) Dominant color frequency histogram.

Table 5.4: Color Patches detected from the Fujifilm IS PRO samples

\begin{tabular}{llll}
\hline Sample & Dim & Patches & id \\
\hline S1 & $995 \times 515$ & 4 & F7, J8, J9, K2 \\
S2 & $620 \times 950$ & 4 & F7, H7, J9, K2 \\
S3 & $453 \times 691$ & 6 & F7, G7, I7, J8, J9, K2 \\
S4 & $725 \times 321$ & 2 & F7, J9 \\
S5 & $185 \times 1406$ & 9 & E7, F7, G7, H7, H8, I8, J8, J9, K2 \\
Support & & 9 & E7, F7, G7, H7, H8, I8, J8, J9, K2 \\
P1 & $222 \times 204$ & 12 & D8, F2, F7, F8, G7, G8, H7, H8, I7, I8, J8, K2 \\
P2 & $252 \times 126$ & 11 & D8, E7, F2, F7, F8, G8, H7, H8, J7, J8, K2 \\
P3 & $177 \times 88$ & 10 & D8, F2, F7, F8, G7, G8, H7, H8, I8, K2 \\
P4 & $192 \times 108$ & 12 & D8, F2, F7, F8, G7, G8, H7, H8, I7, J7, J8, K2 \\
P5 & $105 \times 92$ & 12 & D8, F2, F7, F8, G7, G8, H7, H8, I7, J7, J8, K2 \\
P6 & $161 \times 63$ & 12 & D8, F2, F7, F8, G7, G8, H7, H8, I7, I8, J8, K2 \\
P7 & $84 \times 51$ & 12 & D8, E7, F2, F7, F8, G7, G8, H7, H8, J7, J8, K2 \\
Pigment & & 14 & D8, E7, F2, F7, F8, G7, G8, H7, H8, I7, I8, J7, J8, K2 \\
Support \& Pigment & & 15 & D8, E7, F2, F7, F8, G7, G8, H7, H8, I7, I8, J7, J8, J9, K2 \\
\hline
\end{tabular}




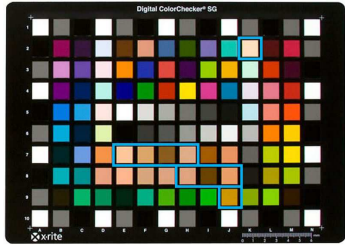

(a)

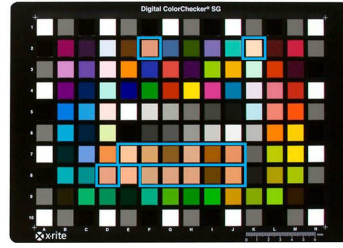

(b)

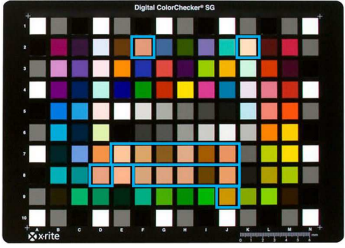

(c)

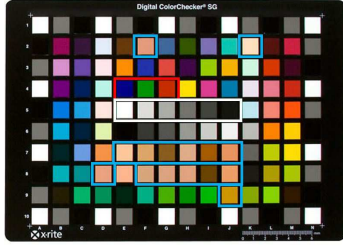

(d)

Figure 5.12: Color patches detected: (a) Support samples; (b) Pigment samples; (c) Support-pigment; (d) KDP training samples.

\subsubsection{Model accuracy assessment of the image-based characterization}

Two different sets of training data were used to build the regression model used in the camera characterization procedure: the FCC set (96 samples) and the KDP (24 selected reported by P-ASK). We conducted a comparison between the results achieved for both training data sets. Table 5.5 shows the basic statistics of the LOOCV residuals of the CIE XYZ predicted values after model adjustment. For the sake of completeness, we also include the residuals of the FCC model applied to the dominant patches in the KDP model, which helps in comparing all data sets. The histograms of the LOOCV CIE XYZ residuals are also displayed in Figure 5.13.

By comparing the residuals of the FCC (with 24 patches) and the KDP model, we find slightly better results in the KDP model, especially in the minimum and the standard deviation statistics (Table 5.5, Figure $5.13 \mathrm{a}$ and b).

Table 5.5: CIE XYZ Residuals of the FCC and KDP models

\begin{tabular}{|c|c|c|c|c|c|c|c|c|c|}
\hline \multirow[b]{3}{*}{ Stats } & \multicolumn{6}{|c|}{ FCC model } & \multirow{2}{*}{\multicolumn{3}{|c|}{$\begin{array}{c}\text { KDP model } \\
(\mathbf{2 4})\end{array}$}} \\
\hline & & (96) & & & (24) & & & & \\
\hline & CIE X & CIE Y & CIE Z & CIE X & CIE Y & CIE Z & CIE X & CIE Y & CIE Z \\
\hline Max & 3.5 & 2.50 & 1.184 & 1.922 & 1.60 & 1.183 & 2.670 & 2.434 & 1.347 \\
\hline Min & -4.985 & -3.852 & -1.612 & -4.985 & -3.852 & -1.587 & -3.523 & -2.591 & -1.090 \\
\hline Mean & 0.017 & 0.010 & 0.006 & -0.428 & -0.359 & -0.156 & 0.013 & 0.008 & 0.003 \\
\hline Std & 1.387 & 1.050 & 0.654 & 1.640 & 1.245 & 0.704 & 1.252 & 0.954 & 0.553 \\
\hline
\end{tabular}

Although the assessment of residuals is very useful to evaluate the predictive capability of the regression model, the computation of the $\Delta E_{a b}^{*}$ color differences is required in colorimetry experiments to rigorously analyze the accuracy achieved after camera characterization. Table 5.6 shows basic color difference statistics computed for the FCC and KDP models. Once again, the $\Delta E_{a b}^{*}$ values obtained by the KDP model show an improvement in terms of colorimetric accuracy when compared with the FCC model. The better results given by the KDP model can be seen graphically in the one-to-one color patches plot displayed in Figure 5.14. All $\Delta E_{a b}^{*}$ in the KDP samples were lower than in the FCC samples, and always under the JND threshold value fixed in 4 CIELAB units (cf. Figure $5.14 \mathrm{~b}$ and a). 


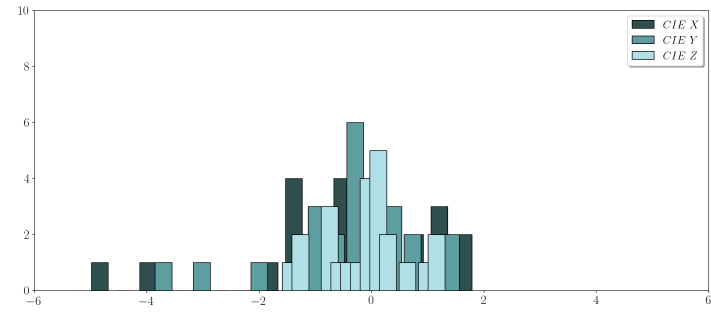

(a)

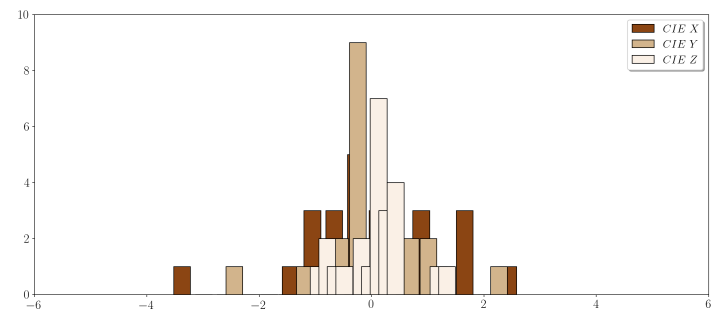

(b)

Figure 5.13: LOOCV CIE XYZ Residuals histogram: (a) FCC (24 patches) (b) KDP (24 patches).

Table 5.6: $\Delta E_{a b}^{*}$ color differences in the FCC and KDP characterization experiments

\begin{tabular}{lccc}
\hline & \multicolumn{2}{c}{ FCC model } & KDP model \\
$\Delta E_{a b}^{*}$ & $\mathbf{( 9 6 )}$ & $\mathbf{( 2 4 )}$ & $\mathbf{( 2 4 )}$ \\
\hline Max & 9.413 & 4.192 & 3.755 \\
Min & 0.322 & 0.497 & 0.179 \\
Mean & 2.107 & 1.868 & 1.518 \\
Std & 1.472 & 1.055 & 0.954 \\
\hline
\end{tabular}

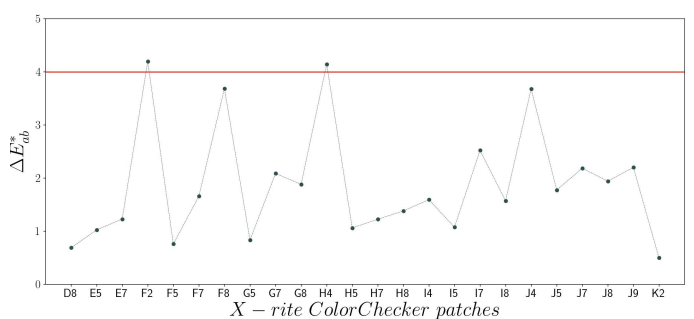

(a)

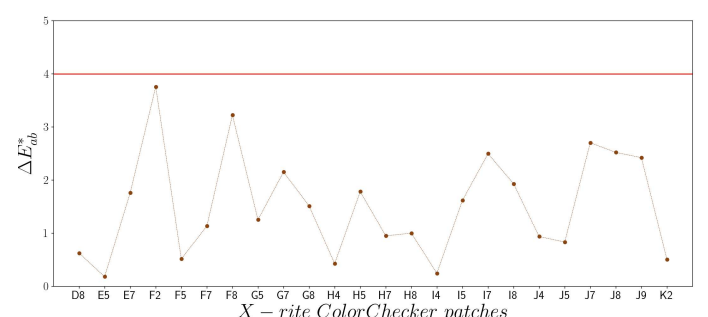

(b)

Figure 5.14: $\Delta E_{a b}^{*}$ color differences: (a) FCC (24 patches); (b) KDP (24 patches).

\subsubsection{Output sRGB characterized images}

Ideally, the final outcome obtained through the characterization process is an image with "colorimetric characteristics." A common approach to preserve such characteristics in digital pictures is the use of the sRGB space, where the RGB data are defined in a physically-based, device-independent color space via transformation functions of CIE XYZ tristimulus values (IEC, 1998). The output sRGB characterized images are shown in Figure 5.15. By observing those sRGB images, it is evident that the results are very satisfying in visual terms, regardless of the training data used for the regression model. 


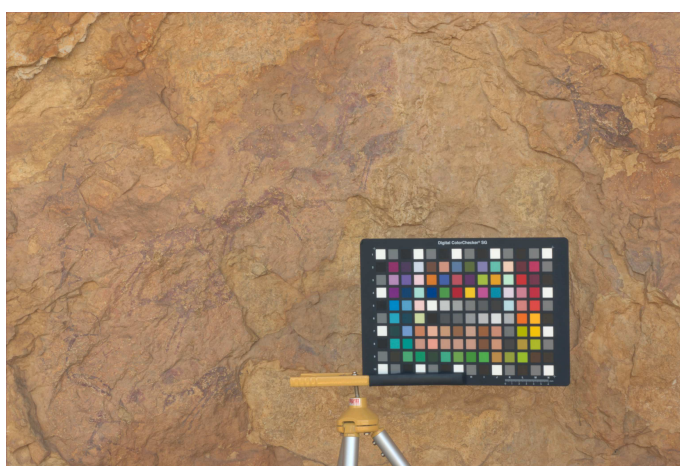

(a)

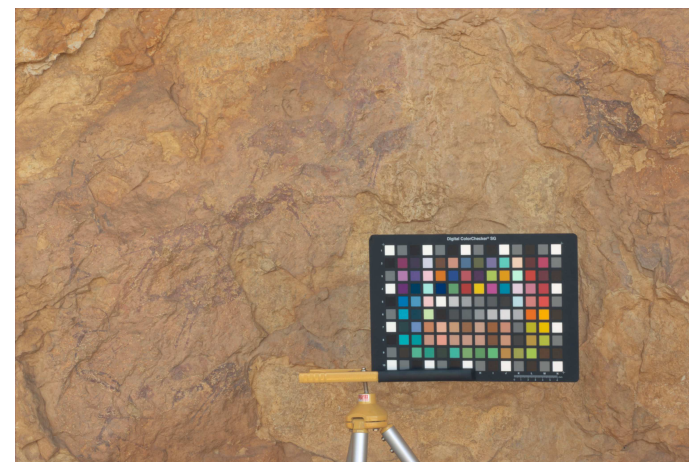

(b)

Figure 5.15: Output sRGB characterized images: (a) FCC training data (96 patches) (b) KDP training data (24 patches).

Although the comparison between the two models confirms that the KDP model performs better in numerical terms, the images provided by the two different training datasets show that both characterization approaches transform successfully RGB data to the CIE XYZ independent color space. It is challenging, even for experienced observers, to perceive any visual differences between the two output images (cf. Figure 5.15 a and $5.15 \mathrm{~b}$ ).

In addition to the numerical computations, we created a $\Delta E_{a b}^{*}$ mapping image with the color differences between the characterized images obtained with the FCC and the KDP training data sets (Figure 5.16).

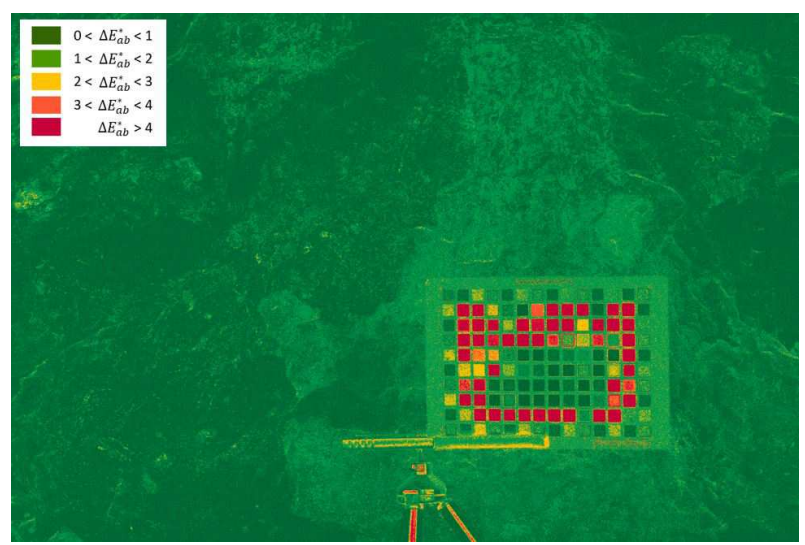

Figure 5.16: $\Delta E_{a b}^{*}$ mapping image (96-24 patches).

The predominant green color present on the difference mapping image indicates that the values of color difference values are less than 2 CIELAB units (see map key in Figure 5.16), which is a very good result in colorimetric terms. Actually, we found higher differences in two cases corresponding to (a) cracks in the rock, where hard shadows are produced (yellow pixels 
with differences between 2 and 3 units), and (b) some patches of the color chart out of the color domain of the scene (differences greater than 2 units coded with yellow, orange and red pixels). Since the focus of the study is on accurate colorimetric analysis of the pigmented areas of the rock art scenes, we can confirm that the P-ASK framework proposed in this study provides very satisfactory results for the camera characterization.

\subsection{Discussion}

The generic aim of clustering applications is the natural grouping of specimens that share similar properties within a data set. In this study, we established the P-ASK framework which leverages the $K$-means technique for automatic training in the selection of a simplified set of color patches, and properly capture the dominant color characteristics of rock art scenes.

Since the $K$-means algorithm is sensitive to the initial approximations given by the user (Bradley and Fayyad, 1998, Limwattanapibool and Arch-int, 2017), a set of preliminary experiments were conducted to set proper values for the number of clusters, the working algorithm and the initialization method. According to our results, the suggested parameters to run $K$-means were appropriate, and the algorithm implemented in the KMeans function of the Scikit-learn module proved to be a valid option for clustering operations as well.

However, the $K$-means algorithm is slow for large data sets, particularly those based on digital images. In our study, we suggest an alternative approach to extract the dominant colors which consists of using a reduced number of image samples clipped from the full image. By using image sampling, the running time was considerably reduced. For instance, run time decreased from 18.2 minutes to 4.9 minutes when using the Fujifilm IS PRO image for $K=24$ clusters (cf. Table 5.3 with Table 5.1 in Section 5.3).

The experiment conducted to identify the effect of randomness on the $K$-means clustering technique showed that the dominant color set returned from multiple runs was highly consistent (MacQueen, 1967). In this regard, Figure 5.7 shows $\Delta E_{a b}^{*}$ color differences less than 1 CIELAB unit for the dominant colors obtained in 100 realizations, with similar negligible results in units of pixel RGB digital values.

Moreover, the capability of P-ASK to identify the dominant colors into the ColorChecker gives very good results (see Table 5.4 and Figure 5.12), and allowed extracting a set of color chips from the chart to create a reduced training data set for camera characterization in a simple and fast way. By using this framework, we proved that the characterization approach based on the KDP training data gives accurate results which parallel those obtained in common practice, that is, using the whole set of color chips for model learning.

Although previous studies recommend the use of large training data sets for camera characterization (Hong et al., 2001, Bianco et al., 2007, Chou et al., 2013, Poljicak et al., 2016), the results obtained with the P-ASK framework show that it is possible to use a reduced number of training samples without loss of accuracy in colorimetric terms. It is worth noting that the CIE XYZ residuals (Table 5.5) and the $\Delta E_{a b}^{*}$ color differences (Table 5.6) obtained using the 
KPD data set are in fact slightly better. In view of the outcome images, it is evident that sRGB characterized images were satisfactorily obtained (Figure 5.15), which is confirmed by the color difference map image where differences under 2 CIELAB units prevail over the whole image, particularly in the area of interest for archaeological applications (Figure 5.16).

As an aside, we would like to note that the specific imaging hardware, i.e. the digital camera used for graphical documentation, is a key point that must be considered prior to characterization. We know from previous research that built-in imaging sensors have a considerable impact on the final outcome. In this sense, we presented the results of the Fujifilm IS PRO camera which is known to work very well, not only in the characterization procedure (Molada-Tebar et al., 2019c), but also in terms of image noise (Marqués-Mateu et al., 2013).

\subsection{Conclusions}

In this chapter, we propose a new P-ASK framework for consistent and accurate archaeological color documentation. The graphic and numeric results obtained after the characterization of rock art images are highly encouraging and confirm that P-ASK is a suitable technique to use in the context of the image-based camera characterization procedure. Our characterization approach based on a reduced set of dominant colors offers several advantages. On the one hand, it implies the reduction of the training set size, and thereby, the computing time required for model training is less than in the regular approach with all color chips. On the other hand, there is an improvement derived from the use of a reduced number of specific color patches, which adjust better to the chromatic range of the scene, and yields better results in the output characterized images. We consider this point as a major contribution to this study.

The tests conducted in our study confirmed that P-ASK is robust to random issues in the $K$-means stage and works very well with a sampling scheme specifically adapted to the elements of interest in the rock art scene. This point leads us to envision future research lines focused on developing specific color charts for archaeological applications, and even for specific cultural heritage objects, monuments and sites. In summary, the results achieved in this study show that P-ASK is a suitable tool for the proper and rigorous use of color camera characterization in archaeological documentation, and can be integrated into the regular documentation workflow with minimum effort and resources. 
6

\section{Discussion}

6.1 Introduction $\ldots \ldots \ldots \ldots \ldots \ldots \ldots \ldots \ldots \ldots \ldots \ldots \ldots \ldots \ldots$

6.2 D65 Illuminant hypothesis $\ldots \ldots \ldots \ldots \ldots \ldots \ldots$

6.3 RAW vs processed image files . . . . . . . . . . . . 101

6.4 Regression model . . . . . . . . . . . . . . . . . . . 104

6.5 Negative CIE XYZ predicted values $\ldots \ldots \ldots 10 \ldots \ldots \ldots$

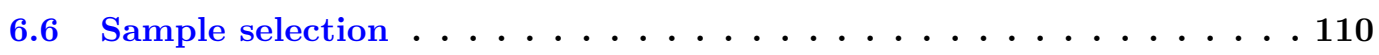

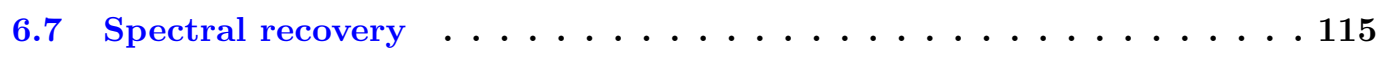




\subsection{Introduction}

Color description from characterized images is a suitable technique in cultural heritage documentation applications due to characteristics such as its low cost, non-destructive data acquisition, ease and speed of collection. The purpose of the colorimetric camera characterization is to transform the input device-dependent data registered by the sensor, generally in RGB format, into a physically-based, independent color space, such as those defined by the CIE, usually the CIE XYZ or any of its derivatives. Although this methodology is not as accurate as an alternative, high precision spot-based approaches using colorimeters, the results obtained in our experiments are accurate enough to be used in rock art documentation as well as in other scientific or industrial applications. A conventional digital camera can be used for rigorous color determination once the camera is properly characterized.

On the other hand, the pyColourimetry software reported in Chapter 2 has proven to be a fundamental piece for the processing of colorimetric and spectral data, and was developed and tested according to the recommendations published by the CIE. It also allows users to achieve full control of the characterization methodological framework established. Thus, through pyColourimetry software users can collect accurate color information, regardless of the camera used in data acquisition, only from digital images captured in a suitable working setup and well-defined colorimetric data for the transformation equations computation. As an aside of the characterization framework proposed, users have the potential to obtain complete output images in the sRGB independent color space (computed from the CIE XYZ predicted values). Considering the outcomes achieved, the results are very satisfactory and promising for the accurate description, recording, rendering, and communication of color in cultural heritage applications among other research fields.

In the following sections, we present further additional comments and discussion regarding the important aspects involved in the camera characterization procedure.

\subsection{D65 Illuminant hypothesis}

The camera characterization procedure must be carried out under specific illuminant conditions. Although it is possible to take direct measurements of the chips from the color checker under D65 illuminant, it is very difficult to work under a controlled light environment in open rock art sites when compared to conducting experiments in lab conditions. So we worked under the hypothesis of D65 illuminant; that is, we extracted the RAW RGB data of the color chips from the image considering D65 conditions during the shot, and we computed the transformation equations using those data.

However, a problem associated with the use of digital images is that the RGB data captured by the sensor are device-dependent, that is, changes during the photographic shot imply changes in the original digital values stored, and consequently in the characterization results. Thus, the factors involved during the photographic shot must be considered, including the camera built-in 
sensor, exposure control, lens, ISO or aperture. Above all, it is required to evaluate the robustness of the characterization process taking into account the lighting variations, that is, the working D65 hypothesis must be confirmed.

To simulate environment illuminant changes in real-work scenarios, we carried out a practical and simple experiment that consisted of characterizing a set of digital images of the same scene taken under different light exposure conditions and analyze the differences. The images correspond to a rock art scene taken in the "Cova Civil" (Figure 1.1). The camera chosen in this case study was the SLR Sigma SD15 camera (Table 1.1). Also, all rock art images were taken including an X-rite ColorChecker@ Digital SD chart for colorimetric reference (Figure 1.3 a).

All the digital images were taken in a very short time-lapse, in RAW format (X3F files for Sigma), under the same lighting conditions (considering the hypothesis of D65 illumination), ISO (100), aperture and focal (f/22, $20 \mathrm{~mm}$ ). To simulate environmental light changes in the scene, we varied the camera exposure parameter: $1 / 5 \mathrm{~s}$ for a normal exposure; $0.6 \mathrm{~s}$ for overexposed; and finally $1 / 25 \mathrm{~s}$ for the underexposed picture (Figure 6.1 ).

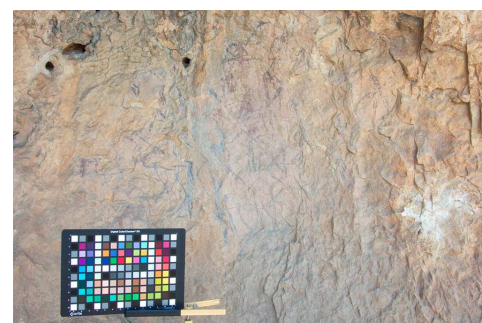

(a)

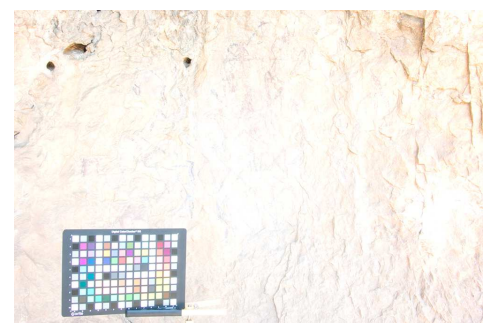

(b)

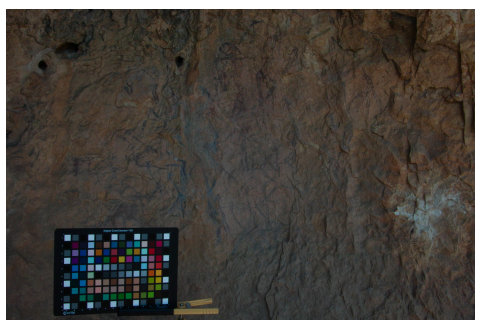

(c)

Figure 6.1: Original RAW images (displayed as JPEG): (a) normal exposure; (b) overexposed; (c) underexposed.

Three images were used to simulate different light conditions, which led to different and specific corrections for each individual image. For model training, we used the RAW RGB data extracted from the color patches, and their theoretical CIE XYZ tristimulus coordinates (D65). We used the whole chip collection (140) from the color checker. The characterization parameters were obtained using a second-order polynomial, which is the most widely used model in characterization. The characterization workflow was carried out using our pyColourimetry software which provides both the CIE XYZ residuals (RMSE) for model assessment and the $\Delta E_{a b}^{*}$ color differences (between theoretical and predicted data) for the colorimetric quality evaluation.

Table 6.1 shows the assessment of the CIE XYZ residual and color difference values achieved after the model fit. Both, the RMSE metrics for the CIE XYZ residuals, and the $\Delta E_{a b}^{*}$ values, confirm that the second-order polynomial used as a regression model for the characterization is adequate (Table 6.1). The best results were obtained for the normal and underexposed image, which gives residuals less than one unit and a $\Delta E_{a b}^{*}$ less than 2.5. Those $\Delta E_{a b}^{*}$ are very satisfactory, since those color difference values are imperceptible by the human eye (Mahy and Van Eycken, 1994). 
Table 6.1: Model fit assessment

\begin{tabular}{|c|c|c|c|c|c|}
\hline Image & Exposure (s) & \multicolumn{3}{|c|}{ CIE XYZ Residuals } & $\Delta E_{a b}^{*}$ \\
\hline Norr & $1 / 5$ & 40 & 0.6368 & 51 & 2.1968 \\
\hline Overexp & 0.6 & 1.0470 & 1.5624 & 0.9257 & 4.9267 \\
\hline Underexposed & $1 / 25$ & 0.7545 & 0.5896 & 0.7104 & 2.4333 \\
\hline
\end{tabular}

As a final outcome, Figure 6.2 displays the set of sRGB output images obtained as a result of the camera characterization. The output sRGB characterized images offer similar results for the three input images. It is not possible to perceive the difference between both in terms of color differences even for experimented observers (cf. Figure 6.2 a with Figure 6.2 c).

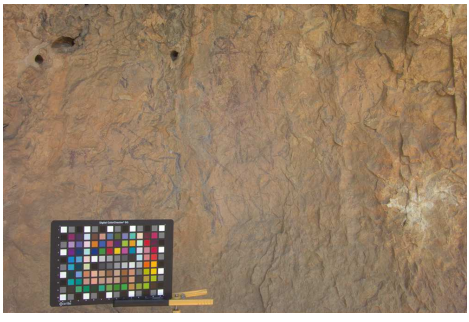

(a)

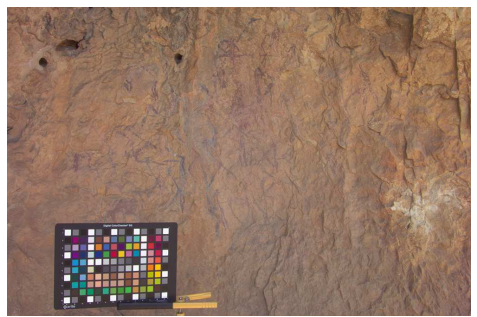

(b)

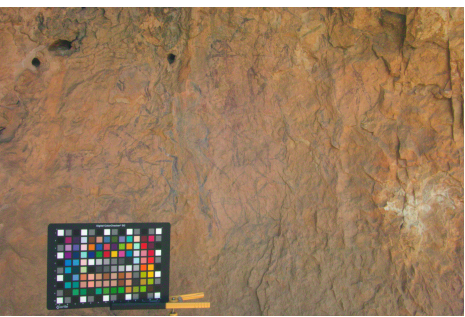

(c)

Figure 6.2: sRGB output images: (a) normal exposure; (b) overexposed; (c) underexposed.

Only for the overexposed image we found $\Delta E_{a b}^{*}$ greater than the JND, around 5 CIELAB units (Table 6.1). However, this is an expected outcome since color perception depends on light. Extreme lighting conditions do modify fundamental colorimetric factors such as the lightness component, but also the chroma component and thereby the color appearance of the object (Figure $6.1 \mathrm{~b}$ ). In fact, it can be clearly observed that the chromatic appearance of the chips from the color chart is far from its normal appearance in the image. Consequently, if the input values extracted from the image lack chromatic quality, the results of the characterization will be substandard as well. Thus, generally, an overexposed image would be discarded in the workflow. In this case, the overexposed image was used only to analyze the behavior of the characterization using a study case in extreme light conditions. However, the sRGB output image obtained from the overexposed image shows similar chromatic range values to those of the outcome from the normal and underexposed images, although the color difference between them can clearly be observed.

In the end, a set of homogeneous images were obtained from the original images taken under different light conditions, which shows the adaptive nature of the image-based characterization. The regression model seems to embed the lighting conditions during the characterization procedure, providing consistent results regardless of the environmental conditions during the shot. Therefore, the color registration in the rock art scenes can be considered correct, and so is the comparison of sets of pictures acquired under different lighting conditions by means of a physically-based sRGB color space. 


\subsection{RAW vs processed image files}

The image-based characterization procedure requires a data set consisting of the RGB digital values acquired by the camera sensor; and their corresponding CIE XYZ tristimulus values of the color chips used as a colorimetric reference. From our experience, we prefer working with RAW format images instead of processed ones, because they avoid a set of complex operations which modify the original RGB data acquired by the camera sensor. Before producing a final color image the RAW data array acquired by the sensor needs to be pre-processed, which includes corrections such as noise reduction, pixel repairing, linearization, white balancing, demosaicing, dark current compensation, and color transform. On the contrary, the RAW output of the camera allows us to work with digital data that resembles the most the physical magnitudes that interact with the sensor. It seems clear that, in order to get more consistent color information, it is recommended to use RAW image files rather than processed or compressed image files (Figure 3.5).

On the other hand, since processed images imply the modification of the original RGB values registered by the camera, it is possible to obtain varying results for the same image depending on the input images. For the same case study, the results can be discarded using processed images, while satisfactory results can be obtained using RAW data. Chapter 3 contains further details about the results achieved using processed data (TIFF format) versus RAW images. The metrics used for the model assessment, that is, the residuals CIE XYZ and the $\Delta E_{a b}^{*}$ color difference values are significantly better. It is clearly observed that relying on RAW data for camera characterization represents a significant improvement results in practical terms (cf. Section 3.4.1 and Section 3.4.2).

We want to note that the case study reported in Chapter 3 was our first practical experiment in camera characterization. It was very helpful in laying out the groundwork for the rest of our research work during the thesis. It made it possible to establish and test a rigorous procedure for the colorimetric camera characterization; analyze the main factors involved, such as the training data, light conditions and the regression model selected; test the pyColourimetry software; and assess the results achieved in the application of the camera characterization procedure to rock art color documentation. From this first experiment, we never used processed images again. Thereafter, we only rely on RAW data, since the results obtained are far superior to those achieved using processed images.

We recommend the use of RAW images since they offer several advantages. The use of RAW data avoids the camera white self-balancing, which is an essential aspect since the color definition is directly related to light conditions. Also, it is not required to apply the non-linearity correction, since RAW data tends to be linear (Section 3.3.3). On the other hand, RAW data are stored using a higher dynamic range of bits, which provides a more wide RGB range to define the colors present on the scene, and consequently a better and precise training dataset specification, which leads to achieving better results after the characterization as well.

However, it is not entirely correct to affirm that RAW data is not subject to any pre-processing operation prior to use. The RAW data captured and stored depends on the sensor manufacturer 
specifications, usually in a binary and complex format. To facilitate the handling of RAW data to users it is required to convert the original RAW data into an easily processable format with minimum pre-processing, in the form of numerical arrays. pyColoutimetry software uses the open-source DCRAW decoder (Coffin, 2020) to get the original data from RAW files as a multidimensional array, which can be read and processed by different Python packages (such as NumPy and SciPy, Table 2.3).

We introduce below an additional real-world example that demonstrates in a very simple and fast visual way that the use of RAW images offers improved, more stable and accurate results instead of the use of processed ones. Figure 6.3 displays a set of rock art painting characterized images using a sample picture in RAW (X3F file) and processed (JPEG format) as inputs. The rock art image was taken using a Sigma SD15 SLR digital camera (Table 1.1) in "Cova dels Cavalls" (Figure 1.2). We used three different polynomial degrees: linear, second and third-order as a regression model. Moreover, Figure 6.4 shows the different $\Delta E_{a b}^{*}$ mapping images computed between the different output sRGB images obtained as a result of the characterization.

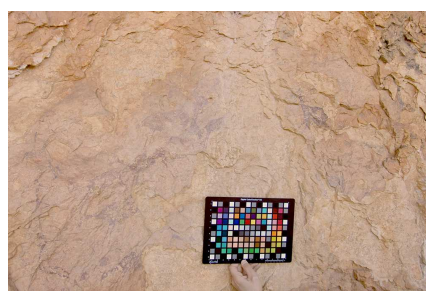

(a)

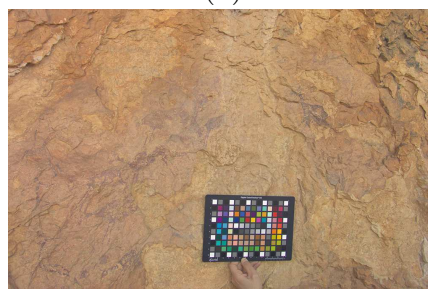

(d)

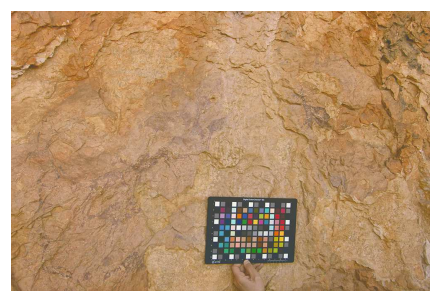

(b)

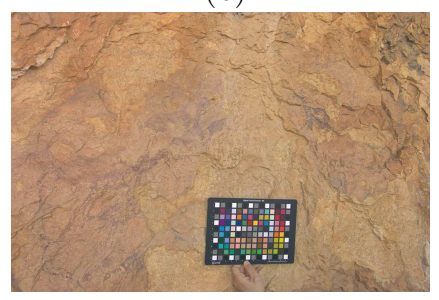

(e)

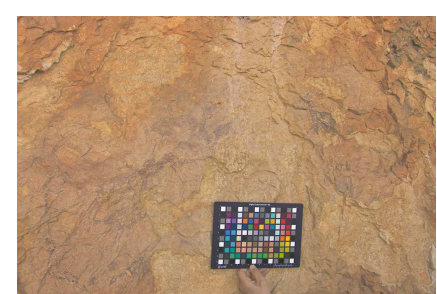

(c)

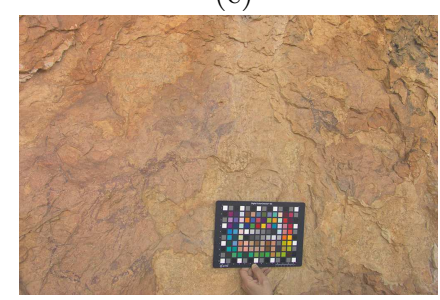

(f)

Figure 6.3: Characterized images: (a,b,c) From JPEG input images; (d,e,f) From RAW input images; (a,d) Linear; (b,e) 2nd; (3,f) 3rd. order

The characterized images from the JPEG images provide the less homogeneous results compared with the outcome from the RAW input data. It is clearly observed that the linear regression model for the processed image gives the worst results in colorimetric terms (cf. Figure $6.3 \mathrm{a}$ with $\mathrm{b}$ and $\mathrm{c}$ ). Even inexperienced observers immediately notice the different appearance of the output linear image compared to the higher-order models. These results were expected, since processed RGB data are not linear. Therefore, in this case, the application of the non-linearity correction is required (Section 3.3.3). On the contrary, it can be observed that the results derived from RAW images hardly show any difference (cf. Figure $6.3 \mathrm{~d}$, e and $\mathrm{f}$ ). The linear model offers satisfying results for RAW data, due to the fact that RAW RGB data tends to be linear. 


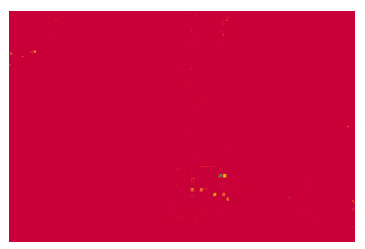

(a)

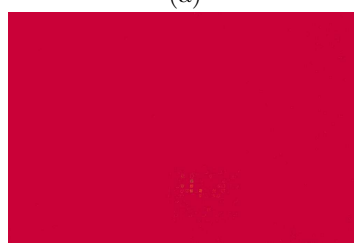

(c)

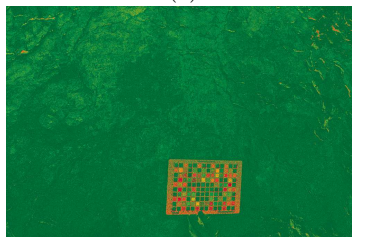

(f)

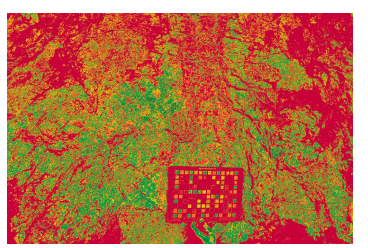

(b)

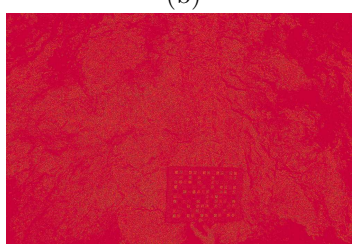

(d)

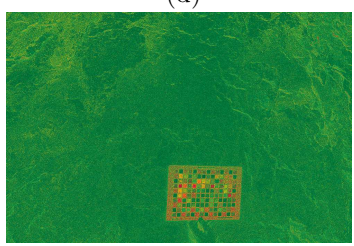

(g)
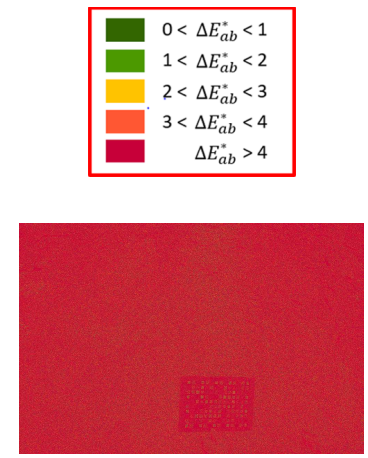

(e)

Figure 6.4: $\triangle E_{a b}^{*}$ mapping images: (a) JPEG 2nd - JPEG 1st; (b) JPEG 2nd - JPEG 3rd; (c) X3F 1st - JPEG 1st; (d) X3F 2nd - JPEG 2nd; (c) X3F 3rd - JPEG 3rd; (f) X3F 2nd - X3F 1st; (g) X3F 2nd - X3F 3rd.

In this regard, the computed $\Delta E_{a b}^{*}$ mapping images are very useful, as they allow us to confirm our conclusion about that the RAW images provided the most stable and homogeneous results. First of all, the results achieved from RAW images are very similar regardless of the order of the polynomial model used for the characterization. The green pixels are predominant in the mapping images computed, which indicate color difference less than 2 CIELAB units (that is, unnoticeable for human vision). Only color differences greater than the JND (4 CIELAB units) are obtained for some patches on the color checker (Figure $6.4 \mathrm{f}$ and $\mathrm{g}$ ).

On the contrary, the $\Delta E_{a b}^{*}$ mapping images computed for the processed characterized images confirm once again that they give the worst results. Red pixels predominate, which indicates $\Delta E_{a b}^{*}$ values great that the JND. The results obtained show that the characterized images vary depending on the polynomial degree used providing more unequal outcomes (cf. Figure 6.4 a and b), whereas the RAW data have the contrary behavior. Also, computing the $\Delta E_{a b}^{*}$ mapping images between the second-order polynomial model from the RAW image and the processed characterized images, shows a clear evidence of the disparity of the results depending on whether the input image is RAW or a processed one (Figure $6.4 \mathrm{c}, \mathrm{d}$ and e). Thus, the use of RAW images is highly recommended instead of processed images regardless of their format. 


\subsection{Regression model}

The accuracy of the results achieved through the camera characterization procedure depends on factors such as the proper selection of the working color spaces (input/output data), the model adjustment capacity, the training data size and the colorimetric properties of the color samples used. The best characterization performance is achieved in the transformation between the device-dependent RGB color space provided by the camera sensor and the independent CIE XYZ color space (Westland et al., 2012).

As a regression model, we used a second-order polynomial, which is widely used for characterization to define the transformation equations between the input and output color spaces. Indeed, the comparison between a common second-order polynomial model and a Gaussian process reported in Chapter 4 confirms that the use of polynomial models offers accurate colorimetric results and keeps the process simple (Molada-Tebar et al., 2019c). Thus, although the GP offers slightly better results, we recommend the use of the second-order polynomial as a regression model for characterization purposes.

However, it should be noted that the residual errors after the regression decrease as the degree of the polynomial model used is increased (Hong et al., 2001). Thus, the question that arises now is why limit the regression model to second-order polynomials, and not use a higher-order polynomial model. This is an important aspect to keep in mind in color science and color adjustments, since the results achieved can become unacceptable in a practical way after the characterization if we use polynomial models with a high number of terms.

In our practical experiments, we used two different metrics in order to evaluate the results obtained after the camera characterization: the CIE XYZ residual values, and the $\Delta E_{a b}^{*}$ color difference. The residuals are a reliable indicator of the goodness of the adjustment. On the other hand, the $\Delta E_{a b}^{*}$ values provide information about the colorimetric quality reached. However, the output sRGB image obtained from the characterization procedure should always be checked.

The model assessment metrics can indicate satisfying results, although they are not necessarily evidence of proper results. It is possible to obtain low residual values, showing that the model fits well with the input training data, and even lower average color differences than JND can be obtained. Nevertheless, even when the results indicate high accuracy, it is possible to find output sRGB image that does not make colorimetric sense, that is, the appearance of the sRGB image does not correspond to the reality that we perceive.

In order to evaluate the effect of the polynomial degree in the characterization procedure, we conducted an experiment consisting of characterizing a digital image by varying the degree of the polynomial regression model from linear to seventh order. The image corresponds to a rock art scenario taken in the "Cova dels Cavalls" (Figure 1.2). In this case, the digital camera chosen was a Sigma SD15, which allows registering pictures in RAW format (Table 1.1).

Figure 6.5 shows the histograms of the CIE XYZ residual values achieved for the first six polynomial models applied. It is observed that all of those polynomial models fit well to the data, have a suitable behavior for characterization purposes and provide satisfying results. 


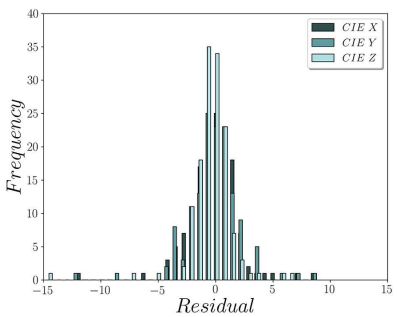

(a)

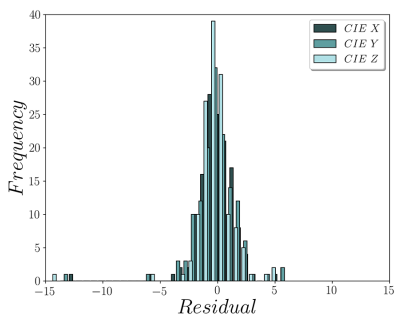

(d)

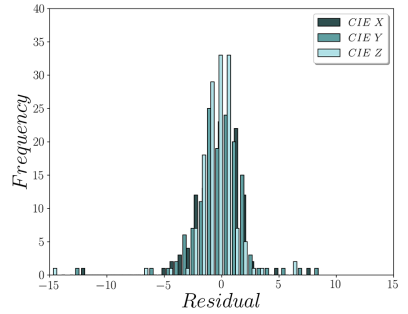

(b)

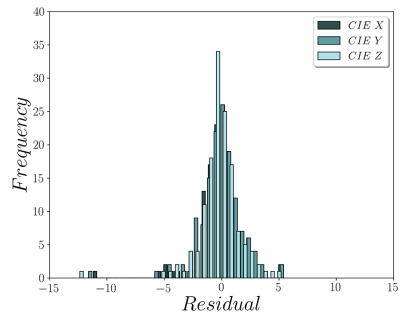

(e)

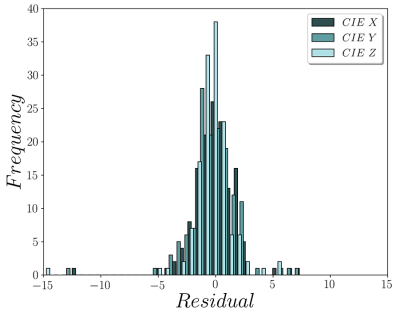

(c)

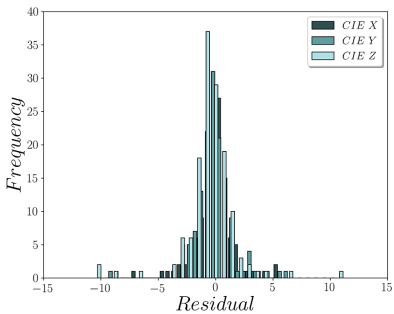

(f)

Figure 6.5: CIE XYZ residuals: (a) Linear; (b) 2nd; (c) 3rd; (d) 4th; (e) 5th; (f) 6th.

On the other hand, Table 6.2 shows the $\Delta E_{a b}^{*}$ color differences values computed. As we expected, the $\Delta E_{a b}^{*}$ values decrease as the degree of the polynomial increases, but only for the first five models. Regression models based on polynomials higher than five degrees do not offer satisfactory results in colorimetric terms, although they provide lower residuals. Consequently, according to the values calculated for color differences, the best result is provided by the fifth-order polynomial regression model applied. However, would we reach the same conclusion by analyzing the sRGB output images obtained as a result of the characterization?

Table 6.2: $\Delta E_{a b}^{*}$ values for the different polynomial models

\begin{tabular}{lrrrrrr}
\hline & 1st & 2nd & 3rd & 4th & 5th & 6th \\
\hline Max & 20.365 & 13.477 & 10.126 & 6.380 & 7.595 & 109.153 \\
Mean & 3.170 & 2.733 & 2.407 & 2.177 & 2.012 & 4.580 \\
Min & 0.294 & 0.167 & 0.449 & 0.260 & 0.197 & 0.350 \\
\hline
\end{tabular}

Figure 6.6 displays the original image and the output sRGB characterized images obtained from the different polynomial degree used during the process. At first view, the images obtained as a result of the first four polynomial models provide outcomes with a similar appearance. Also, the use of polynomials above degree six should not be employed (Figure $6.6 \mathrm{~g}$ and $\mathrm{h}$ ). Although the residual and the $\Delta E_{a b}^{*}$ values obtained are acceptable, observing the characterized image it seems clear that must be discarded (cf. Figure $6.6 \mathrm{f}$ with Figure $6.6 \mathrm{~b}, \mathrm{c}, \mathrm{d}$ and e). 


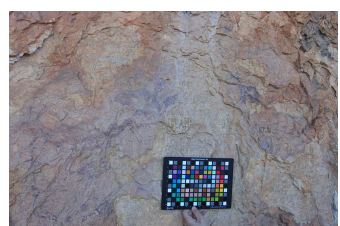

(a)

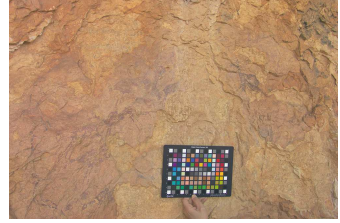

(e)

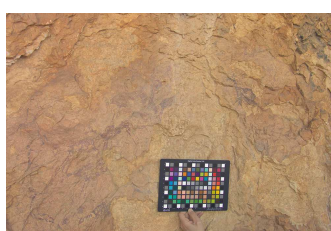

(b)

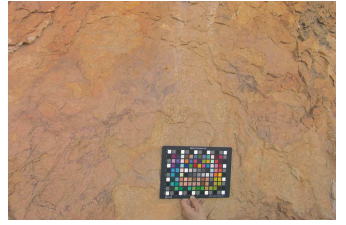

(f)

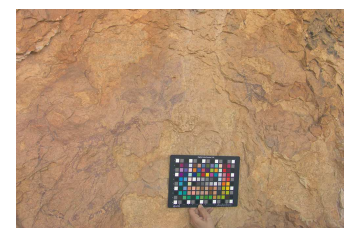

(c)

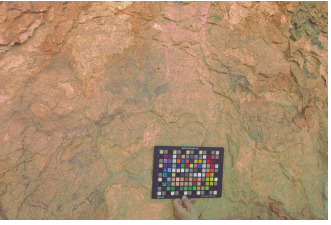

(g)

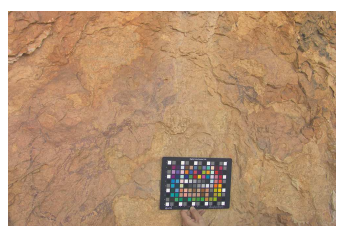

(d)

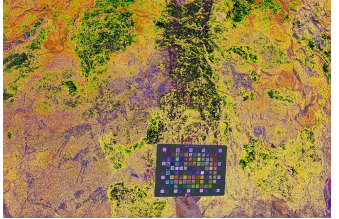

(h)

Figure 6.6: Rock art images: (a) Original RAW (displayed as TIFF); (b,c,d,e,f,g,h) Output characterized images; (b) Linear; (c) 2nd; (d) 3rd; (e) 4th. ; (f) 5th.; (g) 6th.; (h) 7th.

Those affirmations are confirmed by the $\Delta E_{a b}^{*}$ mapping images computed between the second-order (which a priori was considered as the best performance) and the rest of the polynomial regression models applied (Figure 6.7). The best performance was achieved for the linear and second-order polynomial model, since the color difference values are for the majority of the pixels in the image less than 2 CIELAB units, imperceptible to human vision. Also, it is clearly observed that as the degree of the polynomial increases the results are worse from a colorimetric point of view, especially for the higher-order.

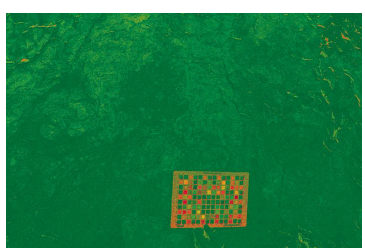

(a)

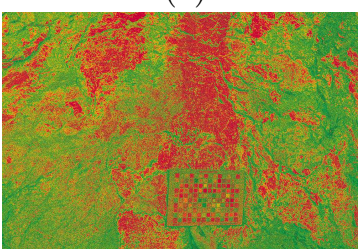

(d)

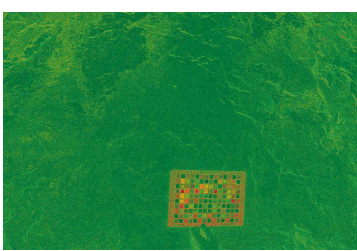

(b)

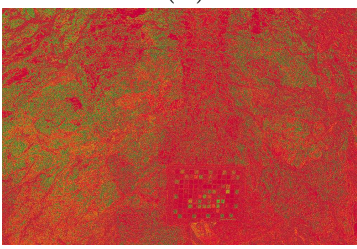

(e)

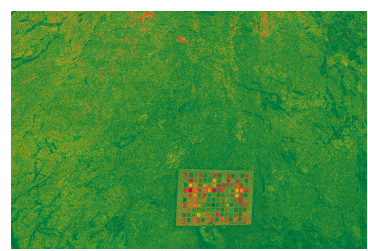

(c)

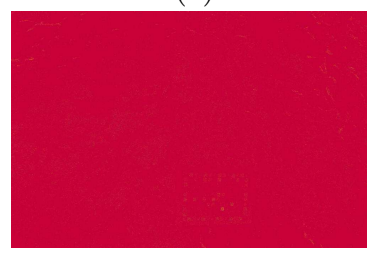

(f)

Figure 6.7: $\Delta E_{a b}^{*}$ mapping images: (a) X3F 2nd - X3F 1st; (b) X3F 2nd - X3F 3rd; (c) X3F 2nd - X3F 4th; (d) X3F 2nd - X3F 5th; (e) X3F 2nd - X3F 6th; (f) X3F 2nd - X3F 7th.

Undeniably, the regression model used in the camera characterization procedure is an essential aspect, since it has a direct impact on the sRGB output images obtained. We highly recommend the use of second-order polynomial models, owing to the fact that they provide satisfactory results 
in real-work scenarios, such as rock art paintings. Thus, it is essential to compute the assessment not only of the capability of the model fit, but the color difference values, and especially the outcome characterized images in order to determine the best performance in practical terms for any specific application.

\subsection{Negative CIE XYZ predicted values}

The broadly regression model used for the camera characterization procedure is based on polynomial models. However, in our experiments, we regularly found that regression models can provide some negative CIE XYZ predicted values after the fit. Thus, although the CIE XYZ color space was developed in order to have non-negative values (Fairman et al., 1997, Wright, 2007, CIE, 2018), we can occasionally get negative values in some complex colorimetric operations (Martínez et al., 2003, Li et al., 2012).

As a final result of the characterization procedure, we obtain an sRGB output image, since most digital devices do not support images in the CIE XYZ color space. Thus, the CIE XYZ values predicted after the regression are encoded as sRGB values in order to be displayed on compatible devices (IEC, 1998). All colors are scaled or clipped so that they do not exceed the gamut boundary defined by the sRGB color space. Thus, negative CIE XYZ values can affect the final output of sRGB values. In fact, for practical purposes, these negative tristimulus values lead to a loss of sharpness in the sRGB output image, as we report below.

From our experience, the number of negative tristimulus values vary depending on the camera built-in sensor, but especially if shadow areas are present in the image. To deal with negative CIE XYZ values, we proposed a simple but effective variable change in order to avoid negative tristimulus values after the model fit. The idea involves entering the square root of both the RGB data and the theoretical CIE XYZ coordinates of the color patches for the regression model. Once the adjustment is computed, we undo the variable change introduced, squaring the predicted CIE $\mathrm{XYZ}$ values obtained. Figure 6.8 displays the scheme for the variable change proposed, which can be understood as a non-negativity constraint imposed on the regression model. Thus, only positive tristimulus values are computed as a result of camera characterization.

We tested the variable change proposed on an image with a rock art specimen taken in "Cova dels Cavalls" (Figure 1.2). The camera used for this experiment was an SLR Canon EOS-1D (Table 1.1), which allows taking images in RAW format (Figure 6.9 a). The regression model chosen in this case for the characterization was a second-order polynomial model, using the 140 color patches from the X-rite ColorChecker@ Digital SD chart as training data.

Detailed analysis allows determining the areas that produce negative CIE XYZ values after applying the model regression in the image, as well as the possible causes for this behavior. Figure 6.10 shows the scatter plot for the coordinates of the color chips used as training data, and the negative tristimulus values obtained as a result of the camera characterization. In addition, Figure 6.11 displays the pixels on the image corresponding to negative values. Comparing both figures, it is clearly observed that these areas correspond with abrupt changes in the relief of the cave wall, and especially in shadow black areas generated by high moisture conditions. 


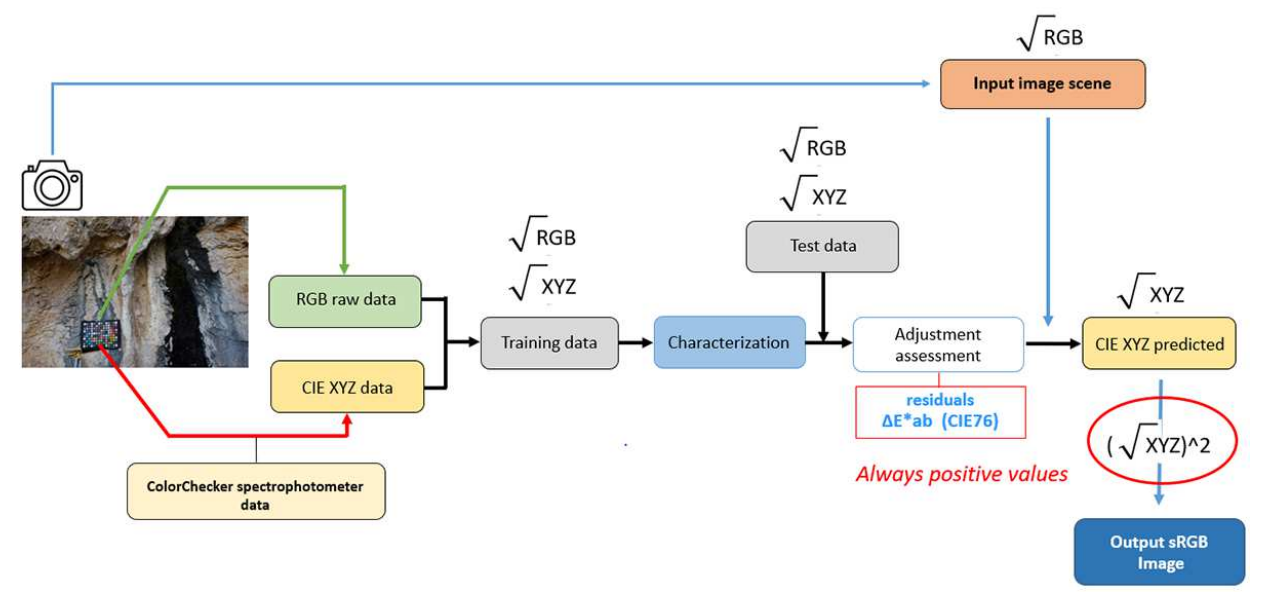

Figure 6.8: Non-negativity constraint scheme for camera characterization procedure.

As a result of the characterization, two sRGB images were obtained (Figure 6.9). The results show an improvement in the characterized image considering the non-negativity constraint. The output image gives lower saturation and higher sharpness, providing as a result a higher quality image (cf. Figure $6.9 \mathrm{c}$ and Figure $6.9 \mathrm{~b}$ ). Although in some cases in order to yield positive CIE $\mathrm{XYZ}$ predicted values a loss of accuracy in terms of color difference can be achieved, the results show the effectiveness of the variable change proposed.

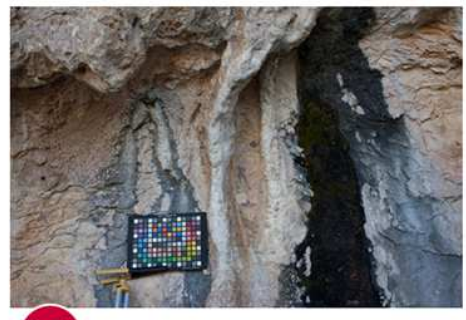

(a)
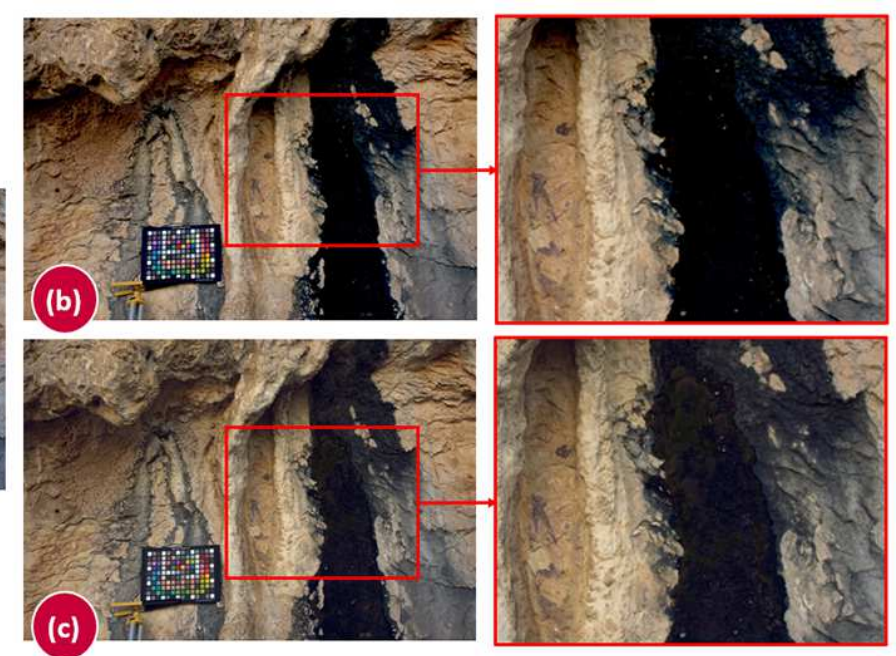

Figure 6.9: Rock art scene images: (a) Original RAW image (displayed as TIFF) ; (b) Output sRGB image; (c) Output sRGB image with the non-negativity constraint. 

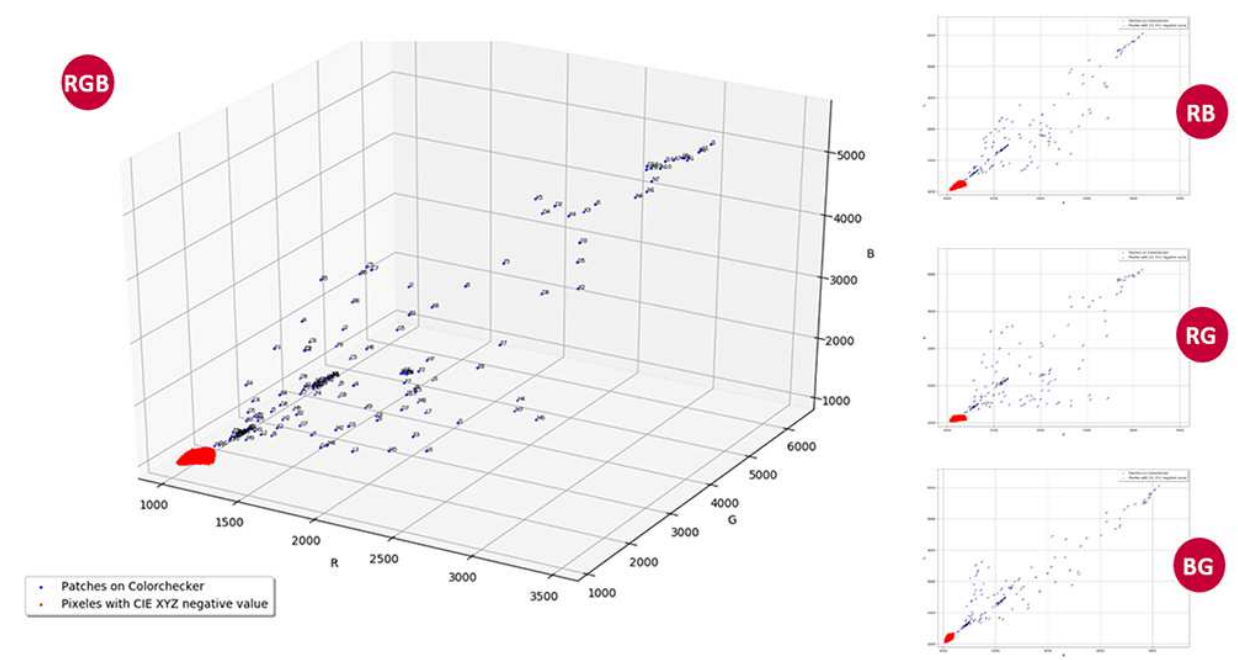

Figure 6.10: RGB negative predicted values scatter plot.

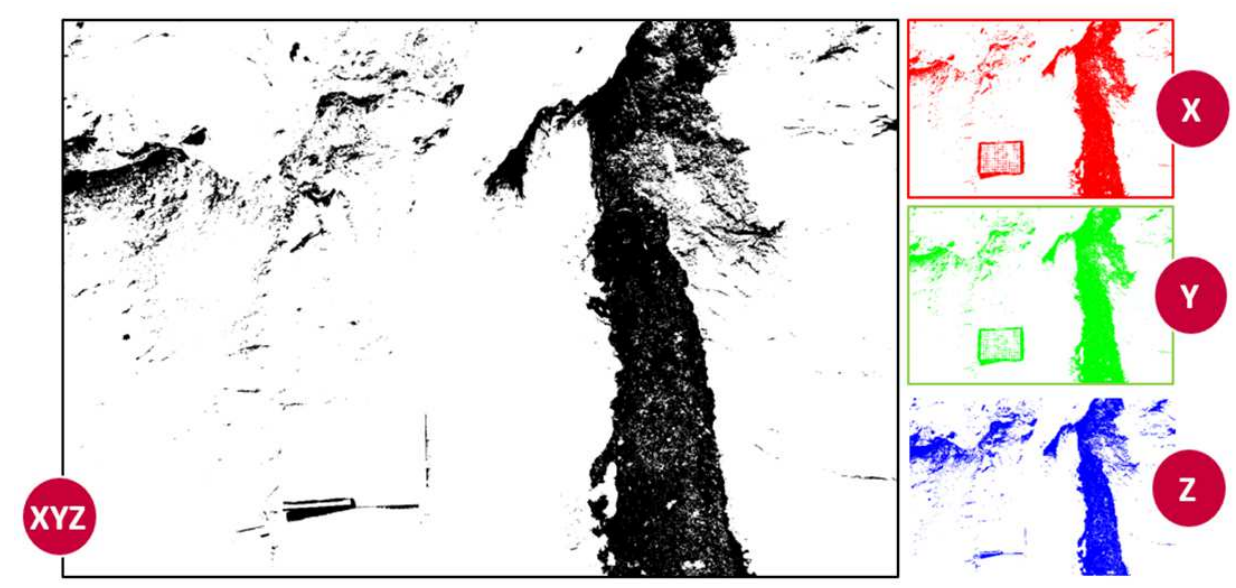

Figure 6.11: CIE XYZ negative predicted values.

In addition, Figure 6.12 displays the $\Delta E_{a b}^{*}$ color difference mapping image between the two sRGB images obtained. It can be observed that the variable change applied does not affect the accurate data achieved in both images, especially on the areas corresponding to colored pigment (cf. detail picture in Figure 6.12). Thus, according to the results obtained we can conclude that this new approach offers accurate results in colorimetric terms, and can be added into characterization procedure in order to avoid negative tristimulus values after the regression. However, we recommend collecting detailed images rather than full scenes, in order to apply the characterization in specific areas where the rock art pigments take up most of the image. Also, we recommend avoiding shadow black areas in pictures. 


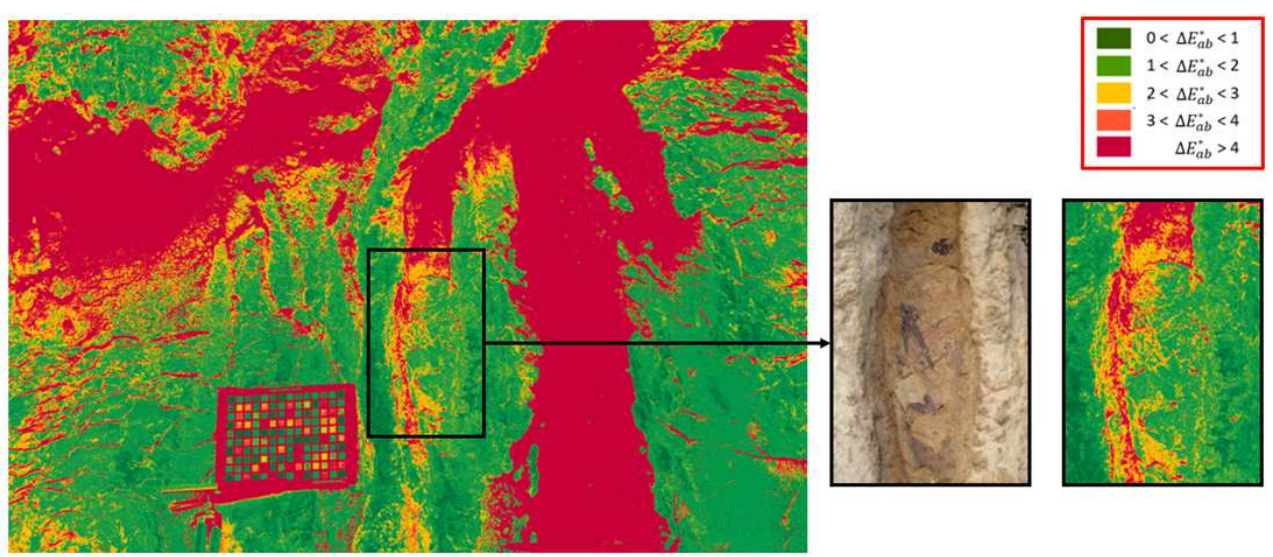

Figure 6.12: $\Delta E_{a b}^{*}$ mapping image.

\subsection{Sample selection}

The camera characterization procedure applied in our practical studies is based on regression models in order to compute the transformation equations between the input/output color spaces considered in the process. An essential aspect for the model accuracy assessment is to relay on a well-established training dataset. Thus, a question to consider is the size of the dataset used for the model training. In our first experiments, we used the whole number of color chips as

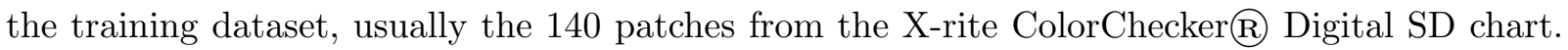
However, satisfying results were found using a selected set of color chips instead of the full color checker.

The effect of the use of different sizes for training datasets was analyzed in previous research (Chang-Rak and Maeng-Sub, 1999, Cheung and Westland, 2002, Cheung et al., 2005, Poljicak et al., 2016). These studies show that accurate results are obtained using around 60-100 color samples. Nevertheless, the accuracy of the model fit did not improve significantly using a set greater than 60 samples (Chang-Rak and Maeng-Sub, 1999, Hong et al., 2001). It is accepted that training datasets composed by 40-60 colored samples offer satisfactory results, thus being a proper number for training samples (Hong et al., 2001). Moreover, Cheung and Westland (2006) present two different algorithms for optimal color sample selection in model training. This study clearly shows how the selection of patches for the training data is another key aspect, since the colorimetric characteristics of the patches used as training samples affect the results achieved in the characterization procedure.

Given the advances produced in recent years by improved built-in sensors, better lens and hardware in digital cameras, it is required to make a review of the effect of the size of the training data on the regression model, not only numerically but also (and especially) graphically in the outcome image provided by the characterization. Thus, the interest is focused on how many samples are required to carry out the characterization with satisfactory and accurate colorimetric results. 
We carried out the analysis on a set of random second-order polynomial regression models, in which we progressively increased the size of the training data. The images correspond to a rock art scene taken in "Cova Civil" (Figure 1.2), from two different SLR cameras: a Fujifilm IS PRO, and a Sigma SD15 (Table 1.1).

For a second-order polynomial model (10 terms), the minimum size for the training data is three samples $(n=3)$. A total of 3450 random models were carried out, 50 models for each $n$ number of samples within the interval $[3,139$, steps $=2]$. The aim is to determine the $n$ number of samples so that the results provided by the regression model are stabilized, by analyzing the mean $\Delta E_{a b}^{*}$ color difference computed for each model. Figure 6.13 displays the mean $\Delta E_{a b}^{*}$ achieved for the random models (a), and the best performance obtained for each set of models (b).

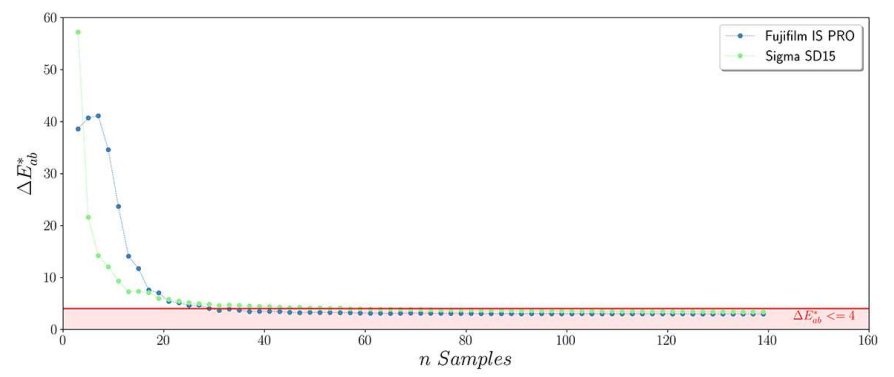

(a)

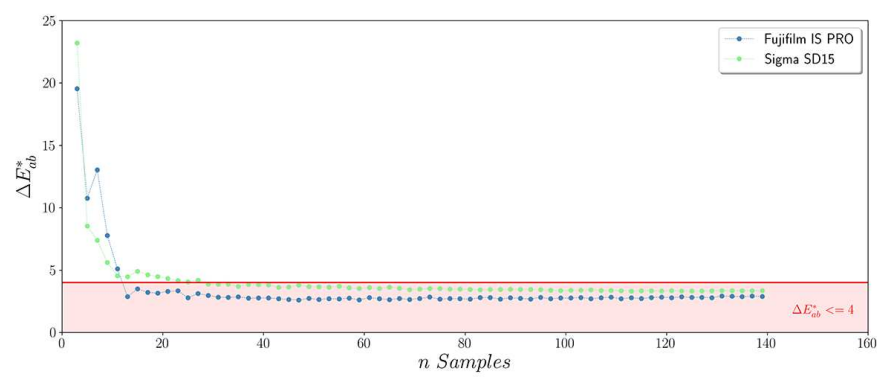

(b)

Figure 6.13: $\Delta E_{a b}^{*}$ : (a) Mean random model; (b) Best random model.

The graphic results achieved show that each camera has a different behavior. Thus, the built-in sensor has a direct influence on the performance of the digital camera during the characterization procedure. In addition, those results confirm that for training data sizes greater than 60 color samples, the results remain stable regardless of the camera used. In fact, it is possible to achieve accurate colorimetric results using 30-40 random color patches. Moreover, the training sample size can be further reduced, since, for a certain combination of a smaller $n$ number of patches, successful results are achieved. Using 13 color samples for the Fujifilm IS PRO camera is enough to achieve $\Delta E_{a b}^{*}$ values less than the JND tolerance, while 29 patches are required for the Sigma (Figure $6.13 \mathrm{~b}$ ). 
The results achieved show that it is not required to use the full number of color chips from the chart for camera characterization, since they introduce redundancy into the regression model. It is possible to use a reduced training data without loss of accuracy, but most importantly, the best performance of the model can be achieved when a well-selected number of samples is introduced into the regression model.

As a first approach we propose the use of skin-tone patches from the reference color checker as an alternative to using the whole color chart, since those are usually the predominant range of colors in rock art scenarios. The characterization performance with skin-tone colors allows the reduction of the training sample size and improves the adjustment to the chromatic range of the scene. In addition, the computing time required for model training is lower than in the regular approach with all color patches.

We tested the characterization of an image from a prehistoric rock art painting scene from the "Cova Civil" shelter (Figure 1.2). The image was taken in RAW format using a Sigma SD15 SLR digital camera (Table 1.1). The regression model used was based on a second-order polynomial model. We applied the characterization using the 140 patches of the X-rite ColorChecker $囚$ Digital SD color chart (ColorChecker dataset), and compared the ColorChecker results with other three additional characterizations based on three subsets of the whole color chart. We name those three subsets 'skin-tone' datasets which are defined with 17 (primary colors [IDs = E4, F4, G4] and skin-tone [IDs = D7, E7, F7, G7, H7, I7, J7, D8, E8, F8, G8, H8, I8, J8]), 28 (skin-tone and grey [IDs = D7, E7, F7, G7, H7, I7, J7, D8, E8, F8, G8, H8, I8, J8]) and 31 patches (primary color, skin-tone and grey).

Graphic and numeric results obtained for the characterized images allow us to confirm that this approach is a suitable alternative for carrying out the characterization of digital cameras. The best results for the image-based characterization were found for the 31 skin-tone dataset. Table 6.3 shows the residuals in CIE XYZ and $\Delta E_{a b}^{*}$ color differences after the second-order polynomial fit using the ColorChecker and skin-tone datasets respectively.

Table 6.3: CIE XYZ residuals and $\Delta E_{a b}^{*}$ after camera characterization.

\begin{tabular}{cccccccc}
\hline & \multicolumn{3}{c}{$\begin{array}{c}\text { ColorChecker dataset } \\
{[\mathbf{1 4 0} \text { patches }]}\end{array}$} & \multicolumn{3}{c}{$\begin{array}{c}\text { Skin-tone dataset } \\
{[\mathbf{3 1} \text { patches }]}\end{array}$} \\
\hline & Max. & Mean & Min. & Max. & Mean & Min. \\
residual X & 6.499 & 0.033 & -12.328 & 2.311 & 0.017 & -3.427 \\
residual Y & 7.255 & 0.042 & -12.218 & 1.878 & 0.012 & -2.519 \\
residual Z & 4.923 & 0.046 & -14.814 & 1.436 & 0.011 & -1.283 \\
$\Delta E_{a b}^{*}$ & 11.728 & $\mathbf{2 . 9 8 5}$ & 0.518 & 4.173 & $\mathbf{1 . 7 6 1}$ & 0.348 \\
\hline
\end{tabular}

The results achieved in this test notably improve when we include the primary color patches from the color checker into the training data. The skin-tone colors used for this experiment are 
located in a very specific region on the chromaticity diagram, compared with the whole sRGB gamut, which covers a larger region as shown in Figure 6.14. Therefore, as the primary color patches are located near the sRGB gamut boundary, the predictive capability of the regression model used in the characterization procedure is much greater, fitting better to the input image data and providing more accurate results.

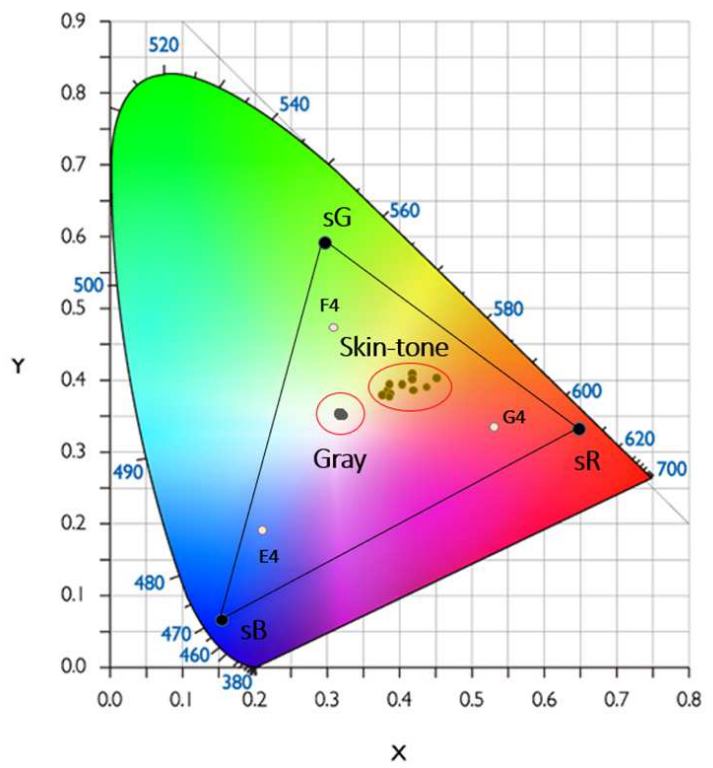

Figure 6.14: 'Skin-tone' dataset patches on CIE chromaticity diagram.

Figure 6.15 shows the output sRGB images obtained using the whole ColorChecker and the 31 skin-tone dataset. Also, a quick way to validate the methodology proposed here is by means of the $\Delta E_{a b}^{*}$ mapping images between the different datasets used for the model training. We set the ColorChecker dataset (140 patches) sRGB image as a color reference, and computed the $\Delta E_{a b}^{*}$ between the 17-, 28- and 31-patch images respectively (Figure 6.16).

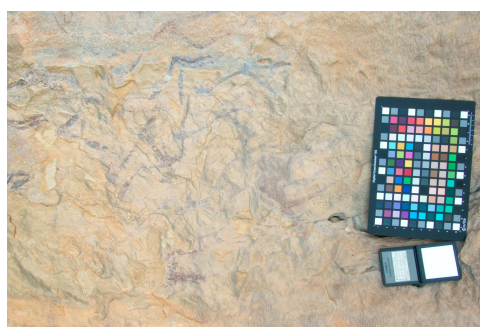

(a)

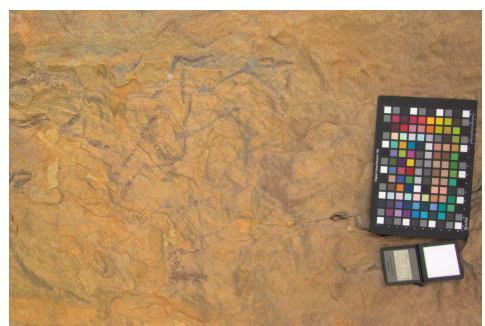

(b)

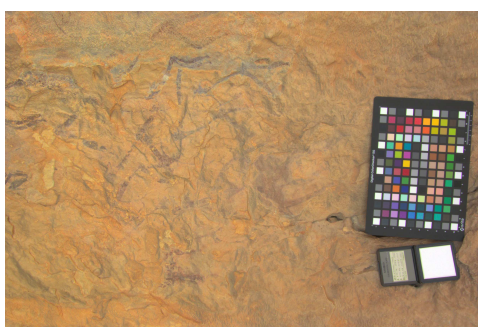

(c)

Figure 6.15: Rock art images: (a) Original Sigma SD15 RAW (viewed as TIFF); (b) ColorChecker [140 patches] characterized image; (c) 'Skin-tone' dataset [31 patches] characterized image. 
We obtained satisfactory results regardless of the data used in the characterization (whole ColorChecker or skin-tone datasets). The mean $\Delta E_{a b}^{*}$ color differences values were less than 4 units for both training datasets, that is, less than the JND (Table 6.3). It is hard for the observer to perceive visual differences between the output sRGB images from both models (cf. Figure 6.15 $\mathrm{b}$ and $\mathrm{c}$ ). Nevertheless, better results were found for the skin-tone dataset, with lower residuals and $\Delta E_{a b}^{*}$ values (Table 6.3).

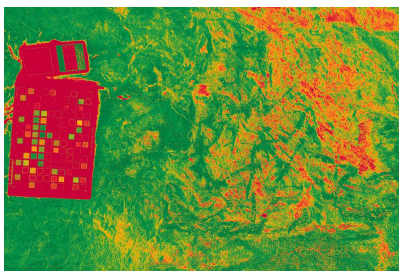

(a)

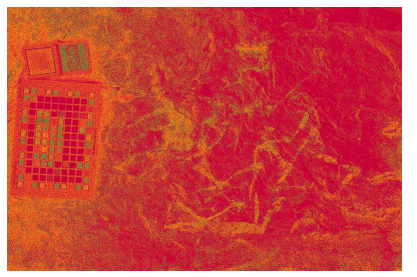

(b)

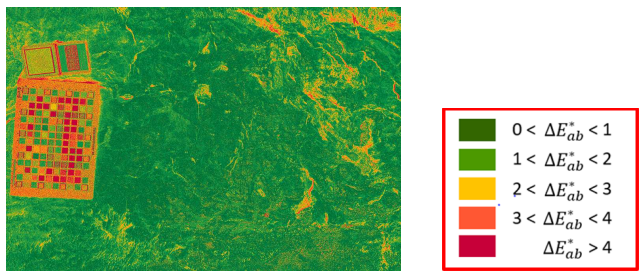

(c)

Figure 6.16: $\Delta E_{a b}^{*}$ mapping images: (a) ColorChecker [140 patches] vs 'Skin-tone' [17 patches]; (b) ColorChecker vs 'Skin-tone' [28 patches]; (c) ColorChecker vs 'Skin-tone' [31 patches].

It is worth noting the differences in the $\Delta E_{a b}^{*}$ mapping images displayed in Figure 6.16. We can conclude that using a subset of 17 patches (primary and skin-tone samples) we can obtain good and acceptable results. Furthermore, the result is improved using the skin-tone dataset, which increases the number of patches from 17 to 31 . Figure $6.16 \mathrm{c}$ shows the predominant green color ( $\Delta E_{a b}^{*}$ less than 2 units), however, Figure $6.16 \mathrm{~b}$ shows quite a different behavior with higher $\Delta E_{a b}^{*}$ values and red colors.

These results clearly show that an increase in the number of sample patches does not intrinsically imply an improvement in the fitness of the output sRGB characterized image. Moreover, even though the two datasets yield good colorimetric results, an improvement is observed when using the skin-tone samples. The results reveal two key aspects in the characterization procedure, specifically the number of samples, and the use of training data near the chromatic range of the scene used in the characterization procedure itself.

This useful practical case study shows the relevance of considering not only the sample size, but also the training samples used for mathematical modeling during the process. In fact, better results are obtained using sample data near the chromatic range of the scene. Instead of a visual selection of the patches by users, in Chapter 5 we propose the novel P-ASK framework to extract a subset of dominant colors from a digital image and automatically identify their corresponding chips in the color chart used as characterizing colorimetric reference. The results reported confirm that the characterization approach based on the P-ASK framework allows not only the reduction of the training sample size but most important a better color adjustment to the chromatic range of the input scene. Thus, an automatic patch selection for camera characterization purposes in archaeological documentation is possible, which can probably be extended to other scientific research fields. 


\subsection{Spectral recovery}

Compared with the CIE XYZ tristimulus values, spectrum data contains much more chromatic information of an object for the correct communication, reproduction and specification of color. Thus, in recent years research has focused on spectral recovery methodologies as an alternative for camera characterization (Amiri et al., 2017, Zhang et al., 2017). Both methodologies have the same objective, that is, the use of consumer-grade cameras for accurate colorimetric measurements since they are a cheaper option instead of using spectrometers. By using digital cameras, instant measurements can be collected for million points (pixels) from an image, capturing colorimetric information for complete scenes in a low-cost and non-invasive way.

However, the problem is that camera manufacturers do not provide sensor sensitivities, which should be measured or estimate by users. The accurate way to measuring the channel sensor sensitivities is by using a monochromator under strict laboratory conditions (Farrell et al., 2017). Although this approach provides the highest accuracy, the drawback is its elevated cost, since it must be carried out in specialist calibration laboratories with high-level equipment. Unfortunately, this option is not available to all users. However, an alternative approach is to estimate the sensor sensitivities given the RGB responses registered by the camera to known spectral stimuli. Thus, the aim of the spectral recovery techniques is to provide the channel spectral sensitivities for a camera sensor.

Different algorithms proposed are reported in the bibliography for spectral reconstruction (Hubelet al., 1994, Dupont, 2002, Heikkinen et al., 2008, Amiri, 2018). In short, the spectral recovery methods are based mainly on two approaches: as a regular regression problem, or as a statistical inversion problem. The main techniques can be summarized as Weiner estimation (Hubelet al., 1994), pseudo-inverse or direct mapping (Valero et al., 2007, Babaei et al., 2011), basis functions (García-Beltrán et al., 1998), single value decomposition (SVD) (Shimano et al., 2007), R-matrix (Zhao and Berns, 2007), and PCA (Fairman and Brill, 2004, Cao et al., 2018). Also, more recently, research is focused on optimized methods to improve the results (Liang and Wan, 2017, Cao et al., 2017, Heikkinen, 2018, Chou et al., 2019, Liang et al., 2019).

Mathematically, the $j t h$ pixel response $P_{i j}$ registered by the camera for the $i t h$ sensor channels (usually, in RGB format) can be expressed as follows (Eq. 6.1):

$$
P_{i j}=\int_{\lambda} E(\lambda) \cdot S(\lambda) \cdot Q(\lambda) \cdot d \lambda=\int_{\lambda} C(\lambda) \cdot Q(\lambda) \cdot d \lambda
$$

where $E(\lambda)$ is the spectral power distribution (SPD), $S(\lambda)$ is the object spectral reflectance and $Q(\lambda)$ the spectral sensitivity. The matrix product of the SPD and the spectral reflectance is known as the $C(\lambda)$ color signal spectral or color stimulus matrix.

The equation 6.1 can be written in matrix form as:

$$
P=C \cdot q
$$


Thus, the $q$ spectral sensitivity for the camera can be computed from the RGB data $(P)$ and the color signal measured $(C)$ under laboratory conditions for the following matrix operation (Eq. 6.3):

$$
q=C^{-1} \cdot P=C^{+} \cdot P
$$

Equation 6.3 assumes that the $\mathrm{C}$ matrix is invertible $\left(C^{-1}\right)$. However, if there are fewer equations than unknowns, the operation can be solved as a pseudo-inverse $\left(C^{+}\right)$problem.

We conducted our first experiment about the spectral recovery method in the same case study reported in Chapter 4. The rock art images were taken in "Cova dels Cavalls" (Figure 1.2). We used two different SLR digital cameras: a Sigma SD15 and a Fujifilm IS PRO (Table 1.1), both in RAW format. The original images are shown in Figure $4.8 \mathrm{a}$ and b. In this case, the spectral reconstruction was computed using the spectral data and the camera responses for the color chips from the X-rite ColorChecker $(\mathrm{R})$ Digital SD chart (140 patches) under the D65 illuminant.

Figure 6.17 shows the channel sensitivities estimates obtained for both SLR digital cameras used in the experiment. It is clearly observed the different behavior of the built-in sensors for each camera. The Fujifilm CCD sensor has the expected performance for the common sensitivities functions. On the contrary, the channel sensitivities functions for the CMOS Foveon $\mathbb{R}$ X3 sensor show its peculiarities as a trichromatic sensor, which records the RGB response independently without any interpolation.

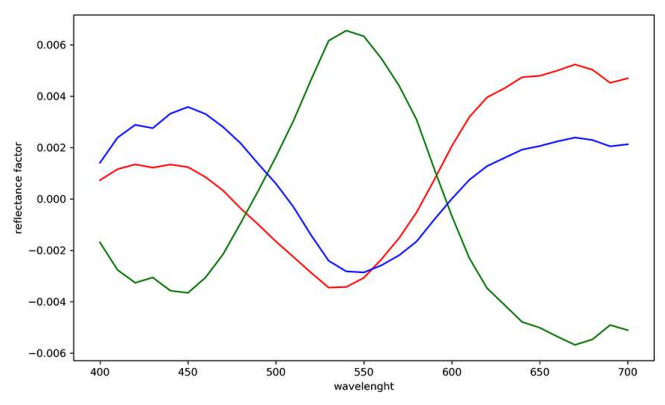

(a)

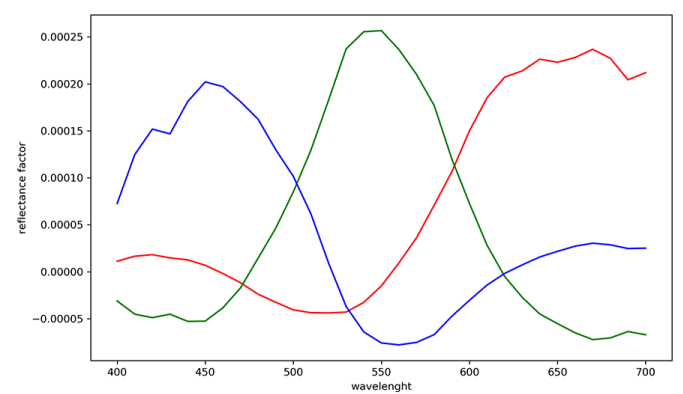

(b)

Figure 6.17: Channel spectral sensitivities: (a) Sigma SD15; (b) Fujifilm IS PRO

Figure 6.18 displays the images created from the spectral recovery method, which can be compared with the outcome provided by the regression models applied for the characterization procedure reported in Chapter 4, that is, a second-order polynomial and the GP model as well. The output sRGB images achieved from the spectral reconstruction method applied are very satisfying, with a mean $\Delta E_{a b}^{*}$ values lower than the JND (4 CIELAB units) for both cameras. The similar final appearance achieved for all the images shows the good performance of the different methods. 


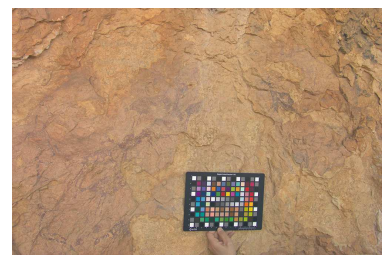

(a)

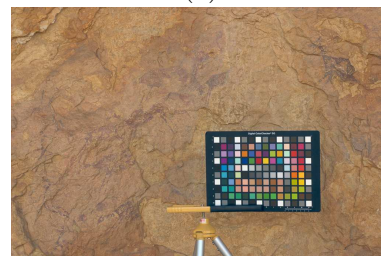

(d)

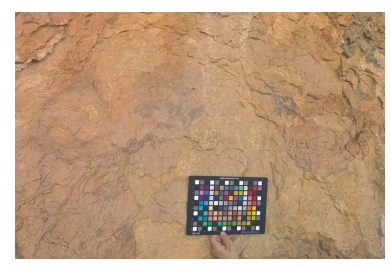

(b)

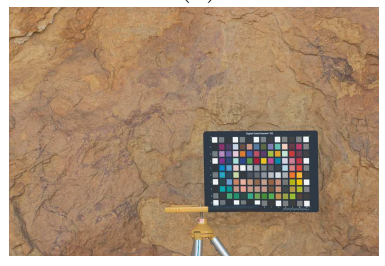

(e)

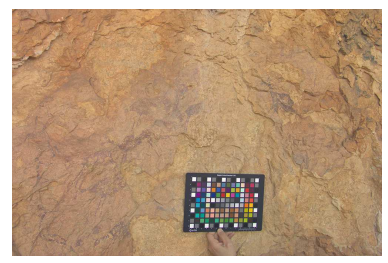

(c)

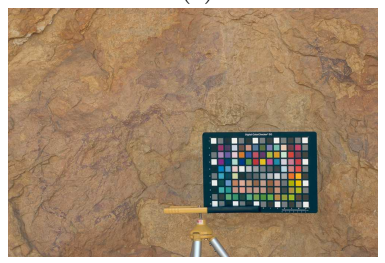

(f)

Figure 6.18: sRGB output images: (a,b,c) Sigma SD15; (d,e,f) Fujifilm IS PRO; (a,d) Second-order polynomial model; (b,e) GP; (c,d) Spectral recovery.

Moreover, it is interesting to analyze the performance of the spectral recovery technique and compare this technique with the outcome provided by the regression models used in camera characterization. Figure 6.19 shows the $\Delta E_{a b}^{*}$ color difference mapping images between the different methodologies applied. The mapping images show the excellent results achieved from the spectral recovery, which provides very similar behavior to that of the second-order polynomial model for the characterization of both cameras (Figure $6.19 \mathrm{a}, \mathrm{b}$ ). Although all the methodologies provide good results, the GP model can be considered as the less recommended option given the results, especially for the Sigma camera (cf. Figure 6.19 b with a, and 6.19 d with c).

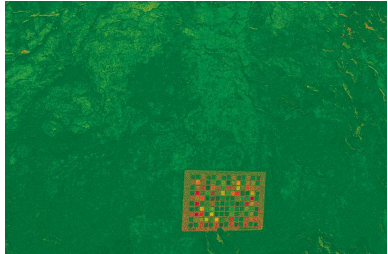

(a)

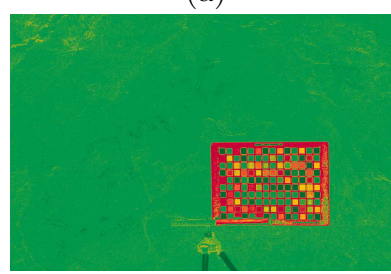

(c)

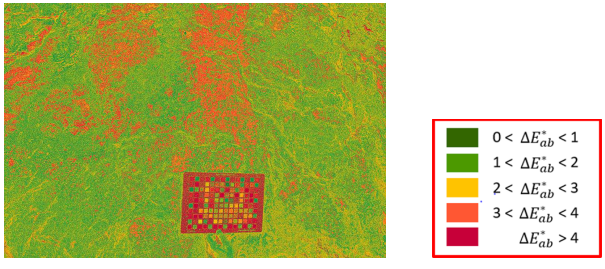

(b)

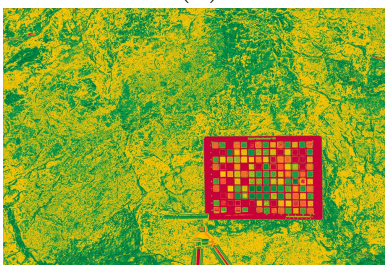

(d)

Figure 6.19: $\Delta E_{a b}^{*}$ color difference mapping images: (a,b) Sigma SD15; (c,d) Fujifilm IS PRO; $(\mathrm{a}, \mathrm{c})$ Spectral recovery - Second-order polynomial model; (b,d) Spectral recovery - GP model. 



\section{7}

\section{Conclusions}

7.1 Initial objectives set and questions raised $\ldots \ldots \ldots \ldots \ldots \ldots \ldots$

7.1.1 Question: Is the camera characterization a suitable procedure for cultural heritage documentation and preservation tasks? . . . . . . . . . 120

7.1.2 Question: Could the GP model provide better results as a regression model for camera characterization? . . . . . . . . . . . . . . . . 121

7.1.3 Question: Is the full set of chips from the color checker needed for model learning in the characterization procedure? . . . . . . . . . . . 121

7.2 Key findings and research contribution . . . . . . . . . . 122

7.3 Remaining research challenges . . . . . . . . . . . . . 124 


\subsection{Initial objectives set and questions raised}

Archaeological rock art documentation and preservation tasks are an intricate and challenging process, where the proper color measurement is an essential aspect that requires the utmost rigor. A correct color specification allows us not only to describe a historical object in its current state but provides useful information about the origin and aging of the pigments used. Complete, exhaustive, reliable and accurate information is required, so that international and national organizations, the heads of cultural heritage conservation agencies and researchers can take specific and adequate actions for the study, description, preservation and occasional restoration of our historical assets.

The problem raised is that classical color recording methods applied in rock art painting have been based on visual observations, supported by color charts as chromatic reference. However, since those methods depend on subjective interpretation procedures and the observer experience, the results obtained can be contradictorily subjected to lack of accuracy. It is evident that verbal expressions are insufficient and cannot be used to communicate color information, unless they are supported by additional accurate techniques for the color specification.

On the contrary, the physical description of color avoids subjective definitions provided by vague color names, as color can be described into independent color spaces, such as those defined by the CIE. In this regard, the colorimetric camera characterization framework reported in this thesis has proved to be a suitable approach for the correct color measurements from digital images.

We tested the methodology on a set of prehistoric rock art painting images captured with a number of digital cameras with different built-in sensors. The outcomes obtained are satisfactory and very promising for proper color specification in cultural heritage studies. Final sRGB images were obtained, in a well-known, independent and physically-based color space, regardless of the digital camera used. Therefore, the main aim of this thesis has been satisfactorily achieved, and the initial questions raised have been answered as well (Chapter 1, Section 1.3). The image-based camera characterization framework proposed, which was integrated into the pyColourimetry software, is rigorous, low-cost and non-invasive, characteristics that make it a suitable technique for cultural heritage applications.

A concise response to the initial questions posed is provided in the following subsections.

\subsubsection{Question: Is the camera characterization a suitable procedure for cultural heritage documentation and preservation tasks?}

Absolutely. The results achieved show that the image-based camera characterization offers accurate colorimetric results, and proves to be a suitable methodology for the accurate registration of color (Chapter 3). The characterization workflow proposed is based on objective methods that are independent of the observer experience. It combines the direct method, based on colorimetric measurements, and the indirect method, using digital RAW images with color information. 
Moreover, the process has been successfully implemented into the pyColourimetry software, which covers all the fundamental colorimetric aspects published by the CIE (Chapter 2). The camera characterization is a suitable technique for cultural heritage documentation in general and rock art in particular because it is low-cost, non-invasive and avoids the subjectivity interpretation of the observer.

Thus, from the camera characterization procedure users can obtain accurate colorimetric information of complete scenes in a physically-based color space, independent of the device, allowing to compare images acquired from different devices since they are referred to the same color space (either CIE XYZ, or sRGB).

\subsubsection{Question: Could the GP model provide better results as a regression model for camera characterization?}

Different mathematical models to characterize digital devices are reported in the literature, but the most widely used are those based on polynomial transformations. Our practical experiments confirmed this point by applying a second-order polynomial model which is enough to achieve accurate results in colorimetric terms (Chapter 2).

We proposed a novel approach based on a Gaussian process in order to improve the characterization results, since the GP are powerful and flexible non-parametric models for multivariate nonlinear functions. The outcome images achieved demonstrated that although both models provide accurate results, the colorimetric characterization based on the GP provides better results, with lower values for the CIE XYZ residuals and $\Delta E_{a b}^{*}$ color differences (Chapter 4).

However, in practice, we recommend the use of a second-order polynomial model, because it is a simpler model for computing and provide very satisfying results.

\subsubsection{Question: Is the full set of chips from the color checker needed for model learning in the characterization procedure?}

No, the use of the whole set of patches from the color chart is not required, since they introduce redundancy into the regression model. In fact, accurate results can be obtained using a reduced and well-selected sample set as training data for the characterization model. We discuss this topic in Chapter 5, and the additional comments provided in Section 6.6. Furthermore, the results achieved confirmed that not only is possible to reduce the training data size without loss of accuracy, but also the intrinsic colorimetric characteristics of the color samples used as training during the characterization procedure have an effect on the accuracy achieved.

Thus, each image should be analyzed separately, so that the color chips to be used as training data adjust better to the chromatic range of the scene. In this sense, the proposed P-ASK framework based on clustering techniques is a suitable alternative for an automatic selection of patches for the characterization process. 


\subsection{Key findings and research contribution}

Given the relevance of accurate color measurement in rock art painting, the approach by means of the colorimetric camera characterization framework reported in this research confirms that it is a suitable technique for the correct color recording, which allows users to register the color as close to reality as possible to understand the true cultural heritage site conditions.

The results achieved confirm that the assumed starting hypotheses were correct, and the actions undertaken have been adequate to achieve the objectives. The process of documentation through the colorimetric characterized image sensors allows rigorous cataloging of heritage, together with the possibility of making a reliable reproduction of the color. Thus, it is possible to rely on characterized digital images that allow right color rendering, and provides very useful information for color description and communication, aspects which are essential in cultural heritage labors.

Quite a few advantages make the camera characterization procedure a highly applicable and rigorous technique suitable for archaeological documentation tasks, particularly in rock art in particular. The use of consumer-grade cameras for accurate colorimetric measurements is a cheaper option compared with the use of spectrometers; it is non-invasive; reduces subjectivity in the study and interpretation of rock art specimens; allows the acquisition of RGB data from complete scenes (millions of pixels) and increases the accuracy in graphic and color definition of objects.

Archaeological documentation greatly benefits from this colorimetric framework. However, the applicability of the camera characterization procedure can be extended to other scientific fields, where the color measurement plays a decisive role. With the image-based camera characterization approach proposed, users can collect rigorous color information regardless of the device used in the data acquisition, with the only requirement of a number of digital images including a color chart and colorimetric measurements. Furthermore, this process is not restrictive and can be used in combination with other common surveying techniques used for the study and conservation of archaeological sites such as laser scanning or photogrammetric techniques. Thus, the combined use of those technologies will allow an improvement in the automation of identification and representation of pigments with maximum reliability in field surveys, 3D models and reconstructions.

In addition, we have observed that it is frequent to use enhanced images in rock art related web sites, usually intended to inform users or just for an on-line catalog. However, those images without any rigorous color treatment are far from its real appearance, and therefore do not transport the user as if they were in situ observing the archaeological site. Therefore, we are confident that the outcome images provided by the camera characterization will be very useful to include in cultural heritage geoportals, or web pages for public or private organizations related to cultural heritage documentation or preservation. 
In short, the main conclusions reached in this thesis can be itemized as follows:

- Methodology: The camera characterization procedure proves to be a suitable methodology for accurate color specification in cultural heritage applications.

- Software: The pyColourimetry software is rigorous; takes into account the most important CIE technical colorimetric aspects; and covers each one of the phases for the methodology proposed.

- Regression model: GP models can be used to improve the results achieved in characterization. However, regression models based on second-order polynomial gives accurate and satisfying results.

- Training data: It is possible to achieve better results when the training data are well-selected, i.e., when they are nearest to the scene chromatic range. In this regard, the P-ASK framework based on the $K$-means algorithm is a suitable method for the automatic patch selection for camera characterization.

- Additional key factors: Some essential factors to be considered during the characterization are: the working color spaces; the camera sensor; the data training size and characteristics; and the regression model used.

Thus, according to the results achieved in this research, we make the following further recommendations:

- Best results for the transformation equations are obtained when the input RGB color space and the CIE XYZ output tristimulus values are used in the regression model.

- The camera built-in sensor has a direct effect on the results provided by the characterization (in terms of noise and colorimetric results). Our experiments show that better results are obtained with the Fujifilm IS PRO camera. Since the proper selection of the camera is a key aspect, we highly recommend conducting a previous analysis of the sensor behavior in order to select the best camera to use for data acquisition.

- The regular color images produced by digital cameras are end products of a set of complex operations, which alter the original color response registered by the sensor. Better results are obtained from RAW images rather than processed ones. In addition, the environmental factors involved in the photographic capture, such as exposure control, lens, ISO or aperture, must be taken into account, since they directly influence the determination of color.

- The image-based characterization based on a second-order polynomial as a regression model provides accurate results, keeps the process simple (in terms of programming), and requires less time for computing as compared with other advanced methods.

- It is not possible to draw the right conclusions only based on residual values provided by the regression model, since the final outcome of camera characterization is a digital image. In addition, the $\Delta E_{a b}^{*}$ color difference values should be analyzed, and even the output characterized image for each practical case. 


\subsection{Remaining research challenges}

This thesis research has focused on establishing a rigorous methodology for the colorimetric camera characterization procedure, and its application on cultural heritage, especially in prehistoric rock art paintings. The characterization through the pyColourimetry software facilitates the data acquisition and measurement tasks as well as the processing of colorimetric samples, getting the polynomial parameters, and applying them to finally obtain the output sRGB image. After the characterization, we can obtain accurate color information, regardless of the camera or sensor used in the data acquisition. The outcome characterized images are very satisfying, promising and highly useful for the proper and accurate specification, registration, dissemination and communication of color in cultural heritage applications.

However, we consider that the framework proposed can be extended to other countless scientific areas where color plays a leading role such as medicine, industry, textile or quality assessment to name a few. In addition, although many of the basic and some of the advanced colorimetry concepts have been applied to reach the main objectives set in this thesis, there are still many aspects and unanswered questions to be studied in further detail. Can the characterization process be further optimized and automated? In some calculations, the effectiveness of Python is limited, as it requires a lot of processing time. Can Python scripts be made more efficient? Thus, it seems highly recommendable for future research to analyze some additional aspects related to programming, the process of methodology, or the development of specific instruments and tools for color measurement.

The following are some of the recommended future research lines related to the camera characterization:

- Python code optimization: OOP modules; migrate the most computationally demanding process to a high performance language such as $\mathrm{C}$ to improve the coding efficiency; integrate full automated process (such as the color check detection, and data extraction).

- LED illumination.

- Problem with the sRGB reduced gamut. Test alternative output color spaces (such as eciRGB).

- Behavior of CATs in the characterization procedure.

- Assessment of the characterization of mobile devices (smartphones).

- Spectral recovery advanced techniques.

- Development of a specific color chart for specific cultural heritage applications, such as archaeological, rock art painting, objects, monuments and sites.

- Design of a portable and low-cost instrument for color measurement, which integrates a colorimeter, digital camera sensor and illumination. 


\section{References}

Adobe. Camera RAW. Available online: https://helpx.adobe.com/camera-raw/using/ supported-cameras.html (accessed on 5 February 2020).

Adobe. Photoshop. Available online: https://www.adobe.com/products/photoshop.html (accessed on 5 February 2020).

Amiri, M.M.; Fairchild, M.D. Use of spectral sensitivity variability in reflectance recovery from colorimetric information. J. Opt. Soc. Am. A 2017, 34, 1224-1235. [CrossRef]

Amiri, M.M.; Fairchild, M.D. A strategy toward spectral and colorimetric color reproduction using ordinary digital cameras. Color Res. Appl. 2018, 43, 675-684. [CrossRef]

Argyll. Argyll Color Management System Home Page. Available online: http://www.argyllcms.com/ (accessed on 5 February 2020)

Arthur, D.; Vassilvitskii, S. k-means++: The advantages of careful seeding. In Proceedings of the eighteenth annual ACM-SIAM symposium on Discrete algorithms 2007, 1027-1035. [url]

Babaei, V.; Amirshahi, S.H; Agahian, F. Using weighted pseudo-inverse method for reconstruction of reflectance spectra and analyzing the dataset in terms of normality. Color Res. Appl. 2011, 36, 295-305. [CrossRef]

Balasubramanian, R. Device Characterization. In Digital Color Imaging Handbook; CRC Press: Boca Raton, Florida, USA, 2003; pp. 270-384.

Barbero-García, I.; Lerma, J.L.; Mora-Navarro, G. Fully automatic smartphone-based photogrammetric 3D modelling of infant's heads for cranial deformation analysis. ISPRS J. Photogramm. Remote Sens. 2020, 166, 268-277. [CrossRef]

Barrile, V.; Fotia, A.; Bilotta,G.; De Carlo, D. Integration of geomatics methodologies and creation of a cultural heritage app using augmented reality. Virtual Archaeol. Rev. 2019, 10, 40-51. [CrossRef]

Behnel, S.; Bradshaw, R.; Citro, C.; Dalcin, L.; Seljebotn, D.S.; Smith, K. Cython: The best of both worlds. Computing in Science \& Engineering 2011, 13, 31-39. [CrossRef]

Bergman, TJ.; Beehner, JC. A simple method for measuring colour in wild animals: Validation and use on chest patch colour in geladas (Theropithecus gelada). Biol. J Linn. Soc. 2008, 94, 213-240. [CrossRef]

Bernardo, J.; Berger, J.; Dawid, A.; Smith, A. Regression and classification using Gaussian process priors. Bayesian Stat. 1998, 6, 475-501. 
Bholowalia, P.; Kumar, A. EBK-means: A clustering technique based on elbow method and k-means in WSN. Int. J. Comput. Appl. 2014, 105, 17-24.

Bianco, S.; Gasparini, F.; Russo, A.; Schettini, R. A New Method for RGB to XYZ Transformation Based on Pattern Search Optimization. IEEE Trans. Consum. Electron. 2007, 53, 1020-1028. [CrossRef]

Bianco, S.; Schettini, R.; Vanneschi, L. Empirical modeling for colorimetric characterization of digital cameras. In Proceedings of the 2009 16th IEEE International Conference on Image Processing (ICIP), Cairo, Egypt, 2009, 1, 3469-3472. [CrossRef]

Bianco, S.; Schettini, R. Two new von Kries based chromatic adaptation transforms found by numerical optimization. Color Res. Appl. 2010, 35, 184-192. [CrossRef]

Bishop, C.M. Pattern Recognition and Machine Learning; Springer: Berlin/Heidelberg, Germany, 2013.

Blanco-Pons, S.; Carrión-Ruiz, B.; Lerma, J.L. Augmented reality application assessment for disseminating rock art. Multimedia Tools and Applications 2019, 78, 10265-10286. [CrossRef]

Boochs, F.; Bentkowska-Kafel, A.; Degrigny, C.; Karaszewski, M.; Karmacharya, A.; Kato, Z.; Picollo, M.; Sitnik, R.; Trémeau, A.; Tsiafaki, D. Colour and Space in Cultural Heritage: Key Questions in 3D Optical Documentation of Material Culture for Conservation, Study and Preservation. Int. J. Herit. Digit. Era 2014, 3, 11-24. [CrossRef]

Bottou, L.; Bengio, Y. Convergence properties of the k-means algorithms. Advances in neural information processing systems 1995, 585-592.

Boyd, CE.; Marín, FM.; Goodmaster, C.; Johnson, A.; Castaneda, A.; Dwyer, B. Digital Documentation and the Archaeology of the Lower Pecos Canyonlands. Virtual Archaeol. Rev. 2016, 3, 98-103. [CrossRef]

Bradley, P.; Fayyad, U.M. Refining Initial Points for K-Means Clustering. ICML 1998, 91-99.

Brill, M.H. Do Tristimulus Values have Units? Color Res. Appl. 1996, 21, 310-313.

Brooks, S.; Gelman, A.; Jones, G.; Meng, X. Handbook of Markov Chain Monte Carlo; CRC Press: Boca Raton, FL, USA, 2011.

Bunting, P.; Clewley, D.; Lucas, R. M.; Gillingham, S. The remote sensing and GIS software library (RSGISLib). Computers $\&$ geosciences 2014, 62, 216-226. [CrossRef]

Burney, SMA.; Tariq, H. K-means cluster analysis for image segmentation. Int. J. Comput. Appl. 2014, 96, 1-5.

BYK. Smart-chart Color Software. Available online: https://www.byk.com/en/instruments/products/ index $\cdot$ php? $a=2 \& b=17 \& c a l$ ibration=\&certification=\&action=\&change_language=en_US (accessed on 4 February 2020).

Cabrelles, M.; Blanco-Pons, S.; Carrión-Ruiz, B.; Lerma, JL. From Multispectral 3D Recording and Documentation to Development of Mobile Apps for Dissemination of Cultural Heritage. In Cyber-Archaeology and Grand Narratives: Digital Technology and Deep-Time Perspectives on Culture Change in the Middle East; Levy, TE., Jones, IWN., Springer, Cham; 2018; 67-90. [CrossRef]

Cai, X.; Langtangen, H.P.; Moe, H. On the performance of the Python programming language for serial and parallel scientific computations. Scientific Programming 2005, 13, 31-56. [CrossRef] 
Canonical Ltd. Ubuntu operating system. Available online: https://www.ubuntu.com/ (accessed on 10 June 2019).

Cao, B.; Liao, N.; Cheng, H. Spectral reflectance reconstruction from RGB images based on weighting smaller color difference group. Color Res. Appl. 2017, 42, 327-332. [CrossRef]

Cao, Q.; Wan, X.; Li, J.; Liu, Q.; Liang, J.; Li, C. Spectral data compression using weighted principal component analysis with consideration of human visual system and light sources. Optical Review 2016, 23, 753-764. [CrossRef]

Cawley, G.; Talbot, N. Efficient leave-one-out cross-validation of kernel fisher discriminant classifiers. Pattern Recognit. 2003, 36, 2585-2592. [CrossRef]

Cerra, D.; Plank, S.; Lysandrou, V.; Tian, J. Cultural Heritage Sites in Danger - Towards Automatic Damage Detection from Space. Remote Sens. 2016, 8, 1-15. [CrossRef]

Chang-Rak, Y.; Maeng-Sub, C. Colorimetric characterization for digital camera by using multiple regression. In Proceedings of IEEE. IEEE Region 10 Conference, "Multimedia Technology for Asia-Pacific Information Infrastructure", Cheju Island, South Korea, South Korea, 1999; 1, 585-558. [CrossRef]

Chaves, J.C.; Nehrbass, J.; Guilfoos, B.; Gardiner, J.; Ahalt, S.; Krishnamurthy, A.; Unpingco, J.; Chalker, A.; Warnock, A.; Samsi, S. Octave and Python: High-Level Scripting Languages Productivity and Performance Evaluation In 2006 HPCMP Users Group Conference (HPCMP-UGC'06); IEEE: Denver, CO, USA, 26-29 June 2006; pp. 429-434. [CrossRef]

Cheung, V.; Westland, S. Color Camera Characterisation Using Artificial Neural Networks. In Proceedings of the Tenth Color Imaging Conference: Color Science and Engineering Systems, Technologies, Applications, Scottsdale, AZ, USA, 12 November 2002; 1, 117-120. [url]

Cheung, V.; Westland, S.; Connah, D.; Ripamonti, C. A comparative study of the characterisation of color cameras by means of neural networks and polynomial transforms. Color. Technol. 2004, 120, 19-25. [CrossRef]

Cheung, V.; Li, C.; Hardeberg, J.; Connah, D.; Westland, S. Characterization of trichromatic color cameras by using a new multispectral imaging technique. J. Opt. Soc. Am. A 2005, 22, 1231-1240. [CrossRef]

Cheung, V.; Westland, S. Methods for Optimal Color Selection. J. Imaging Sci. Technol. 2006, 50, 481-488. [CrossRef]

Chou, Y.; Luo, M.R.; Li, C.; Cheung, V.; Lee, S. Methods for designing characterisation targets for digital cameras. Color. Technol. 2013, 129, 203-213. [CrossRef]

Chou, TR.; Hsieh, CH.; Chen, E. Recovering spectral reflectance based on natural neighbor interpolation with model-based metameric spectra of extreme points. Color Res. Appl. 2019, 44, 508-525. [CrossRef]

Chudoba, R.; Sadílek, V.; Rypl, R.; Vořechovský, M. Using Python for scientific computing: Efficient and flexible evaluation of the statistical characteristics of functions with multivariate random inputs. Computer Physics Communications 2013, 184, 414-427. [CrossRef]

CIE. A review of Chromatic Adaptation Transforms; Commission Internationale de l'Éclairage: Vienna, Austria, 2004. 
CIE. Colorimetry - Part 4: CIE $1976 L^{*} a^{*} b^{*}$ Colour Space; Commission Internationale de l'Éclairage: Vienna, Austria, 2007.

CIE. Colorimetry-Part 6: CIEDE2000 Colour-Difference Formula; Commission Internationale de l'Éclairage: Vienna, Austria, 2014.

CIE. Colorimetry, 4th Edition; Commission Internationale de l'Éclairage: Vienna, Austria, 2018.

Clewley, D.; Bunting, P.; Shepherd, J.; Gillingham, S.; Flood, N.; Dymond, J.; Lucas, R.; Armston, J.; Moghaddam, M. A Python-Based Open Source System for Geographic Object-Based Image Analysis (GEOBIA) Utilizing Raster Attribute Tables. Remote Sens. 2014, 6, 6111-6135. [CrossRef]

Coffin, D. DCRAW. Available online: https://www.dechifro.org/dcraw/ (accessed on 5 February 2020)

Colantoni, P.; Thomas, J.B.; Hardeberg, J.Y. High-end colorimetric display characterization using an adaptive training set. J. Soc. Inf. Disp. 2011, 19, 520-530. [CrossRef]

Colom, M.; Buades, A.; Morel, J.M. Nonparametric noise estimation method for raw images. J. Opt. Soc. Am. A 2014, 31, 863-871. [CrossRef]

python-colormath. Available online: https://python-colormath.readthedocs.io/en/latest/ (accessed on 5 February 2020)

ColorMine. Available online: http://www.colormine.org/ (accessed on 5 February 2020)

Colour. Available online: https://colour.readthedocs.io/en/develop/index.html (accessed on 5 February 2020)

DCamProf - a digital camera profiling tool. Available online: https://www.ludd.ltu.se/ torger/ dcamprof.html (accessed on 5 February 2020)

del Hoyo-Meléndez, J.M.; Lerma, J.L.; López-Montalvo, E.; Villaverde, V. Documenting the light sensitivity of Spanish Levantine rock art paintings. ISPRS Ann. Photogramm. Remote Sens. Spatial Inf. Sci. 2015, II-5/W3, 53-59. [CrossRef]

del Hoyo-Meléndez, J.M.; Carrión-Ruiz, B.; Riutort-Mayol, G.; Lerma, J.L. Lightfastness assessment of Levantine rock art by means of microfading spectrometry. Color Res. Appl. 2019, 44, 547-555. [CrossRef]

Demster, A.P.; Laird, N.M.; Rubin, D.B. Maximum-likehood from incomplete data via the EM algorithm. J. R. Stat. Soc. Ser. B Methodol. 1977, 39, 1-38.

Domingo, I.; Villaverde, V.; López-Montalvo, E.; Lerma, JL.; Cabrelles ,M. Latest developments in rock art recording: towards an integral documentation of Levantine rock art sites combining 2D and 3D recording techniques. J Archaeol Sci. 2013, 40, 1879-1889. [CrossRef]

Domingo, I.; Carrión-Ruiz, B.; Blanco, S.; Lerma, J.L. Evaluating conventional and advanced visible image enhancement solutions to produce digital tracings at el Carche rock art shelter. Digital Applications in Archaeology and Cultural Heritage 2015, 2, 79-88. [CrossRef]

Dupont, D. Study of the reconstruction of reflectance curves based on tristimulus values: comparison of methods of optimization. Color Res. Appl. 2002, 27, 88-99. [CrossRef] 
Durmus, A.; Moulines, E.; Pereyra, M. Efficient Bayesian computation by proximal Markov chain Monte Carlo: When Langevin meets Moreau. SIAM J. Imaging Sci. 2018, 11, 473-506. [CrossRef]

Eckhard, T.; Valero, E.M.; Hernández-Andrés, J.; Schnitzlein, M. Adaptive global training set selection for spectral estimation of printed inks using reflectance modeling. Appl. Optics 2014, 53, 709-719. [CrossRef]

Elkan, C. Using the triangle inequality to accelerate k-means. In Proceedings of the 20th International Conference on Machine Learning (ICML-03), 2003, 147-153.

FADGI. Federal Agencies Digital Guidelines Initiative. Available online: http://www . digitizationguidelines.gov/ (accessed on 10 June 2019).

Fairman, H.S.; Brill, M.H.; Hemmendinger, H. How the CIE 1931 color-matching functions were derived from Wright-Guild data. Color Res. Appl. 1997, 22, 11-23. [CrossRef]

Fairman, H.S.; Brill, M.H. The principal components of reflectances. Color Res. Appl. 2004, 29, 104-110. [CrossRef]

Farrell, J.; Okincha, M.; Parmar, M. Sensor calibration and simulation. In Proc. SPIE Digital Photography $I V$, San Jose, California, United States, 2008; 6817, 68170R. [CrossRef]

Fernández-Lozano, J.; Gutiérrez-Alonso, G.; Ruiz-Tejada, MÁ.; Criado-Valdés, M. 3D digital documentation and image enhancement integration into schematic rock art analysis and preservation: The Castrocontrigo Neolithic rock art (NW Spain). J. Cult. Herit. 2017, 26, 160-166. [CrossRef]

Files, M.; Griggs, K. Technical Guidelines for Digitizing Cultural Heritage Materials: Creation of Raster Image; Image Rochester: NY, USA, 2016.

Finlayson, G.; Mackiewicz, M.; Hurlbert, A. Color Correction Using Root-Polynomial Regression. IEEE Trans. Image Process. 2015, 24, 1460-1470. [CrossRef]

Forgi, E. Cluster analysis of multivariate data: efficiency vs. interpretability of classifications. Biometrics 1965, 21, 768-769.

Gaiani, M.; Fabrizio,I.A.; Ballabeni, A.; Remondino, F. Securing Color Fidelity in 3D Architectural Heritage Scenarios. Sensors 2017, 17, 2437-2461. [CrossRef]

García-Beltrán, A.; Nieves, J.L.; Hernández-Andrés, J.; Romero, J. Linear bases for spectral reflectance functions of acrylic paints. Color Res. Appl. 1998, 23, 39-45. [CrossRef]

Gareth, J.; Daniela, W.; Trevor, H.; Rober, T. An Introduction to Statistical Learning with Applications in R; Springer: New York, NY, USA, 2013.

Gelman, A.; Carlin, J.B.; Stern, H.S.; Dunson, D.B.; Vehtari, A. Bayesian Data Analysis, 3rd ed.; CRC Press: Boca Raton, FL, USA, 2013.

GIMP. GNU Image manipulation program. Available online: https: //www. gimp. org/ (accessed on 5 February 2020).

Girolami, A.; Napolitano, F.; Faraone, D.; Braghieri, A. Measurement of meat color using a computer vision system. Meat Sci. 2010, 93, 111-118. [CrossRef] 
González-Aguilera, D.; Muñoz-Nieto, A.; Gómez-Lahoz, J.; Herrero-Pascual, J.; Gutierrez-Alonso, G. 3D Digital Surveying and Modelling of Cave Geometry: Application to Paleolithic Rock Art. Sensors 2009, 9, 1108-112\%. [CrossRef]

Green, P.J.; Silverman, B.W. Nonparametric Regression and Generalized Linear Models: A Roughness Penalty Approach; CRC Press: Boca Raton, FL, USA, 1993.

Hamerly, G.; Elkan, C. Alternatives to the k-means algorithm that find better clusterings. Proceedings of the eleventh international conference on Information and knowledge management 2002, 600-607.

Heikkinen, V.; Lenz, R.; Jetsu, T.; Parkkinen, J.; Hauta-Kasari, M.; Jääkeläinen, T. Evaluation and unification of some methods for estimating reflectance spectra from RGB images. J. Opt. Soc. Am. A 2008, 25, 2444-2458. [CrossRef]

Heikkinen, V. Spectral Reflectance Estimation Using Gaussian Processes and Combination Kernels. IEEE Trans. Image Process. 2018, 27, 3358-3373. [CrossRef]

Higuchi, R.; Suzuki, T.; Shibata, M.; Taniguchi, Y.; Gülyaz, M. Digital non-metric image-based documentation for the preservation and restoration of mural paintings: the case of the Üzümlü Rock-hewn Church, Turkey. Virtual Archaeol. Rev. 2016, 7, 31-42. [CrossRef]

Hong, G.; Luo, M.R.; Rhodes, P.A. A study of digital camera colorimetric characterization based on polynomial modeling. Color Res. Appl. 2001, 26, 76-84. [CrossRef]

Hubel, P.M.; Sherman, D.; Farrell, J. A Comparison of Methods of Sensor Spectral Sensitivity Estimation. In Color and Imaging Conference 1994, 1, 45-48. [url]

Hung, P. Colorimetric calibration in electronic imaging devices using a look-up-table model and interpolations. J. Electron. Imaging 1993, 1, 53-61. [CrossRef]

International Color Consortium. ICC profiles. Available online: http://www.color.org/profiles2. xalter (accessed on 5 February 2020)

International Color Consortium. Profiling tools. Available online: http://www.color.org/ profilingtools.xalter (accessed on 5 February 2020)

Institut Cartogràfic Valencià. Visor de Cartografía. Available online: https://visor.gva.es/visor (accessed on 4 November 2019).

IEC. IEC/4WD 61966-2-1: Colour Measurement and Management in Multimedia Systems and Equipment-Part 2-1: Default RGB Colour Space-sRGB; International Electrotechnical Commission: Geneva, Switzerland, 1998.

ImageMagick Studio LLC. ImageMagick. Available online: https://imagemagick.org/index.php (accessed on 5 February 2020).

Ippoliti, E.; Calvano, M. Enhancing the Cultural Heritage Between Visual Technologies and Virtual Restoration. In Digital Curation: Breakthroughs in Research and Practice; Hershey, PA., IGI Global; 2019; pp. 309-348. [CrossRef]

International Organization for Standardization. ISO 12647-2. Graphic technology: Process control for the production of half-tone colour separations, proof and production prints. 2004. 
Iturbe, A.; Cachero, R.; Cañal, D.; Martos, A. Virtual digitization of caves with parietal Paleolithic art from Bizkaia. Scientific analysis and dissemination through new visualization techniques. Virtual Archaeol. Rev. 2018, 9, 57-65. [CrossRef]

Jackman, P.; Sun, D.W.; ElMasry, G. Robust colour calibration of an imaging system using a colour space transform and advanced regression modelling. Meat Sci. 2012, 91, 402-407. [CrossRef]

Johnson, GM.; Fairchild, MD. Visual psychophysics and color appearance. In Digital Color Imaging Handbook; CRC Press: Boca Raton, Florida, USA, 2003; pp. 127-183.

Joydeep, G.; Alexander, L. K-Means. In The top ten algorithms in data mining; CRC press: Boca Raton, FL, 2009; pp. 21-35.

Kanungo, T.; Mount, D.M.; Netanyahu, N.S.; Piatko, C.D.; Silverman, R.; Wu, A.Y.. An efficient k-means clustering algorithm: Analysis and implementation. IEEE Trans. Pattern. Anal. Mach. Intell. 2002, 881-892. [CrossRef]

Kemp, DB. Colorimetric characterisation of flatbed scanners for rock/sediment imaging. Computers 86 Geosciences 2014, 67, 69-74. [CrossRef]

Konica Minolta. CM-600d Spectrophotometer. Available online: https://sensing.konicaminolta.us/ products/cm-600d-spectrophotometer/ (accessed on 4 February 2020).

Konica Minolta. CS-100A colorimeter. Available online: https://sensing.konicaminolta.us/ products/cs-100a-luminance-and-color-meter/ (accessed on 4 February 2020).

Konica Minolta. Data Management Software CS-S10w. Available online: https://https: //www5.konicaminolta.eu/en/measuring-instruments/products/light-display-measurement/ software/cs-s10w/specifications.html (accessed on 4 February 2020).

Konica Minolta. SpectraMagicNX. Available online: https://sensing.konicaminolta.us/products/ spectramagic-nx-color-data-software/ (accessed on 4 February 2020).

Korytkowski, P.; Olejnik-Krugly, A. Precise capture of colors in cultural heritage digitization. Color Res. Appl. 2017, 42, 333-336. [CrossRef]

Lang, H. How much physics does colorimetry really need? Color Res. Appl. 1997, 22, 212-215.

Langtangen H.P.; Cai X. On the Efficiency of Python for High-Performance Computing: A Case Study Involving Stencil Updates for Partial Differential Equations. In Modeling, Simulation and Optimization of Complex Processes, Berlin, Heidelberg, 2008, Springer, pp. 337-357.

Lebrun, M.; Buades, A.; Morel, J.M. A nonlocal bayesian image denoising algorithm. SIAM J. Imaging Sci. 2013, 6, 1665-1688. [CrossRef]

Leon, K.; Mery, D.; Pedreschi, F.; Leon, J. Color measurement in $L^{*} a^{*} b^{*}$ units from RGB digital images. Food Res. Int. 2006, 39, 1084-1091. [CrossRef]

Lerma, J.L.; Navarro, S.; Cabrelles, M.; Villaverde, V. Terrestrial laser scanning and close range photogrammetry for 3D archaeological documentation: the Upper Palaeolithic Cave of Parpalló as a case study. J. Archaeol. Sci. 2010, 37, 499-507. [CrossRef]

Lerma, J.L.; Cabrelles, M.; Navarro, S. Fusion of range-based data and image-based datasets for efficient documentation of cultural heritage objects and sites. ISPRS Ann. Photogramm. Remote Sens. Spatial Inf. Sci. 2015, XL-5/W\%, 277-281. [CrossRef] 
Li, B.; Xu, D.; Xiong, W.; Feng, S. Illumination-independent descriptors using color moment invariants. Opt. Eng. 2009, 48, 027005. [CrossRef]

Li, C.; Perales, E.; Luo, M.R.; Martínez-Verdú, F. Mathematical approach for predicting non-negative tristimulus values using the CAT02 chromatic adaptation transform. Color Res. Appl. 2012, 37, 255-260. [CrossRef]

Liang, J.; Wan, W. Optimized method for spectral reflectance reconstruction from camera responses. Opt. Express 2017, 25, 28273-28287. [CrossRef]

Liang, J.; Xiao, K.; Pointer, M.R.; Wan, X.; Li, C. Spectra estimation from raw camera responses based on adaptive local-weighted linear regression. Opt. Express 2019, 27, 5165-5180. [CrossRef]

Limwattanapibool, O.; Arch-int, S. Determination of the appropriate parameters for K-means clustering using selection of region clusters based on density DBSCAN (SRCD-DBSCAN). Expert Syst. 2017, 34, e12204. [CrossRef]

Lindbloom, B.J. Useful Color Calculators and Spreadsheets. Available online: http://www . brucelindbloom.com/index.html/ (accessed on 5 February 2020)

Liu, Z.; Liu, Q.; Gao, G.; Li, C. Optimized spectral reconstruction based on adaptive training set selection. Opt. Express 2017, 25, 12435. [CrossRef]

Lloyd, S. Least squares quantization in PCM. IEEE Trans. Inf. Theory 1982, 28, 129-137.

López-Menchero, VM.; Marchante, Á.; Vincent, M.; Cárdenas, ÁJ.; Onrubia, J. Combined use of digital nightlight photography and photogrammetry in the process of petroglyphs documentation: The case of Alcázar de San Juan (Ciudad Real, Spain). Virtual Archaeol. Rev. 2017, 8, 64-74. [CrossRef]

Luo, MR.; Cui, G.; Rigg, B. The development of the CIE 2000 color-difference formula: CIEDE2000. Color Res. Appl. 2001, 26, 340-350. [CrossRef]

Luo, MR. Applying colour science in colour design. Opt. Laser Technol. 2006, 38, 392-398. [CrossRef]

MacQueen, J. Some methods for classification and analysis of multivariate observations. Proceedings of the fifth Berkeley symposium on mathematical statistics and probability 1967, 1, 281-297.

Mahy, M.; Van Eycken, L.; Oosterlinck, A. Evaluation of Uniform Color Spaces Developed after the Adoption of CIELAB and CIELUV. Color Res. Appl. 1994, 19, 105-121.

Maietti, F.; Di Giulio, R.; Piaia, E.; Medici, M.; Ferrari, F. Enhancing Heritage fruition through 3D semantic modelling and digital tools: the INCEPTION project. IOP Conf. Ser.: Mater. Sci. Eng. 2018, 364,012089 .

Malacara D. Color vision and colorimetry: Theory and applications.; 2nd ed.; SPIE: Bellingham, Washington, USA, 2011.

Malo, J.; Luque, M.J. ColorLab: the Matlab toolbox for Colorimetry and Color Vision. Univ. València. Available online: http://isp.uv.es/code/visioncolor/colorlab.html (accessed on 5 February 2020)

Marqués-Mateu, Á.; Lerma, J.L.; Riutort-Mayol, G. Statistical grey level and noise evaluation of Foveon X3 and CFA image sensors. Opt. Laser Technol. 2013, 48, 1-15. [CrossRef] 
Martínez, J.A.; Pérez-Ocón, F.; García-Beltrán, A.; Hita, E. Mathematical determination of the numerical data corresponding to the color-matching functions of three real observers using the RGB CIE-1931 primary system and a new system of unreal primaries X'Y'Z'. Color Res. Appl. 2003, 28, 89-95. [CrossRef]

Martínez Valle, R.; Villaverde Bonilla, V. La Cova dels Cavalls en el Barranc de la Valltorta. Monografías del Instituto de Arte Rupestre. Museu de la Valltorta. Tirig. Generalitat Valenciana. 2002.

Martínez-Verdú, F.; Pujol, J.; Capilla, P. Characterization of a digital camera as an absolute tristimulus colorimeter. J. Imaging Sci. Technol. 2003, 47, 279-295. [url]

MathWorks(R). Color. Available online: https://www . mathworks.com/help/images/color.html?s_tid= CRUX_lftnav (accessed on 5 February 2020)

McDonald, R.; Rigg, B. Colour Physics for Industry; 2nd ed.; The Society of Dyers and Colourists: Bradford, UK, 1997.

Melgosa, M.; Alman, D.H.; Grosman, M.; Gómez-Robledo, L.; Trémeau, A.; Cui, G.; García, P.; Vázquez, D.; Li, C.; Luo, M.R. Practical demonstration of the CIEDE2000 corrections to CIELAB using a small set of sample pairs. Color Res. Appl. 2013, 38, 429-436. [CrossRef]

Bureau Metamorfoze. metamorfoze. Available online: https://www.metamorfoze.nl/english (accessed on 10 June 2019).

Microsoft. Windows. Available online: https://www.microsoft.com/en-us/windows/ (accessed on 27 January 2020).

Mohammadi, M.; Nezamabadi, M.; Berns, R.S.: Taplin, L.A. Spectral Imaging Target Development Based on Hierarchical Cluster Analysis. Color and Imaging Conf. 2004, 59-64. []url]

Molada-Tebar, A.; Lerma, J.L; Marqués-Mateu, Á. Software development for colorimetric and spectral data processing: PyColourimetry. In Proceedings of the 1st Congress in Geomatics Engineering, Valencia, Spain, 5-6 July 2017; Volume 1, 48-53. [CrossRef]

Molada-Tebar, A.; Lerma, J.L.; Marqués-Mateu, Á. Camera characterization for improving color archaeological documentation. Color Res. Appl. 2018, 43, 47-57. [CrossRef]

Molada-Tebar, A.; Marqués-Mateu, Á.; Lerma, J.L. Camera Characterisation Based on Skin-Tone Colours for Rock Art Recording. Proceedings 2019, 19, 12. [CrossRef]

Molada-Tebar, A.; Marqués-Mateu, Á.; Lerma, J.L. Correct use of color for cultural heritage documentation. ISPRS Ann. Photogramm. Remote Sens. Spatial Inf. Sci. 2019, IV-2/W6, 107-113. [CrossRef]

Molada-Tebar, A.; Riutort-Mayol, G.; Marqués-Mateu, Á.; Lerma, J.L. A Gaussian Process Model for Color Camera Characterization: Assessment in Outdoor Levantine Rock Art Scenes. Sensors 2019, 19, 4610. [CrossRef]

Molada-Tebar, A.; Marqués-Mateu, Á.; Lerma, J.L.; Westland, S. Dominant Color Extraction with $K$-Means for Camera Characterization in Cultural Heritage Documentation. Remote Sens. 2020, 12, 520 [CrossRef] 
Naveed, K.; Ehsan, S.; McDonald-Maier, K.D.; Ur Rehman, N. A Multiscale Denoising Framework Using Detection Theory with Application to Images from CMOS/CCD Sensors. Sensors 2019, 19, $206-227$. [CrossRef]

Neal, RM. Monte Carlo implementation of Gaussian process models for Bayesian regression and classification. arXiv 1997, arXiv:physics/9701026.

NumPy. Available online: https://numpy.org/ (accessed on 3 February 2020).

Ohno, Y. Spectral Color Measurement. In Colorimetry: understanding the CIE system; John Wiley \& Sons, Inc.: Hoboken, New Jersey, USA, 2007; pp. 101-134.

Ohta, N.; Robertson, A. Colorimetry: fundamentals and applications; John Wiley \& Sons, Inc.: Chichester, UK, 2005.

Ohta, N.; Robertson, A. Measurement and Calculation of Colorimetric Values. In Colorimetry: fundamentals and applications; John Wiley \& Sons, Inc.: Chichester, UK, 2005, pp. 153-174

Olejnik-Krugly, A.; Korytkowski, P. Precise color capture using custom color targets. Color Res. Appl. 2020, 45, 40-48. [CrossRef]

Oliphant TE. Python for Scientific Computing. Comput. Sci. Eng. 2007, 9, 10-20. [CrossRef]

OpenCV. Available online: https://opencv.org/ (accessed on 3 February 2020).

Padín, J.; Martín, A.; Anquela, AB. Archaeological microgravimetric prospection inside don church (Valencia, Spain). J. Archaeol. Sci. 2012, 39, 547-554. [CrossRef]

Pan, Y.; Dong, Y.; Wang, D.; Chen, A.; Ye, Z. Three-dimensional reconstruction of structural surface model of heritage bridges using UAV-based photogrammetric point clouds. Remote Sens. 2019, 10, 1204. [CrossRef]

Pedregosa, F.; Varoquaux, G.; Gramfort, A.; Michel, V.; Thirion, B.; Grisel, O.; Blondel, M.; Prettenhofer, P.; Weiss, R.; Dubourg, V. Scikit-learn: Machine learning in Python. J. Mach. Learn. Res. 2011, 12, $2825-2830$.

Peña, JM.; Lozano, JA.; Larranaga, P. An empirical comparison of four initialization methods for the k-means algorithm. Pattern Recognit. Lett. 1999, 20, 1027-1040. [CrossRef]

Pham, D.T.; Dimov, S.S.; Nguyen, C.D. Selection of K in K-means clustering. Proceedings of the Institution of Mechanical Engineers, Part C: Journal of Mechanical Engineering Science 2005, 219, 103-119. [CrossRef]

Pointer, M.R.; Attridge, G.G.; Jacobson, R.E. Practical camera characterization for colour measurement. Imaging Sci. J. 2001, 49, 63-80. [CrossRef]

Poljicak, A., Dolic, J., Pibernik, J. An optimized Radial Basis Function model for color characterization of a mobile device display. Displays 2016, 41, 61-68. [CrossRef]

Previtali, M.; Valente, R. Archaeological documentation and data sharing: digital surveying and open data approach applied to archaeological fieldworks. Virtual Archaeol. Rev. 2019, 10, 17-27. [CrossRef]

Python Software Foundation. Available online: https://www.python.org/ (accessed on 4 November 2019). 
Python Software Foundation. Graphical User Interfaces with Tk. Available online: https ://docs . python . org/3/library/tk.html (accessed on 03 February 2020).

Qian, Y.; Chen, K.; Nikkanen, J.; Kamarainen, J.K. Recurrent Color Constancy. Proc. IEEE Int. Conf. Comput. Vis. 2017, 1, 5459-5467.

Ramanath, R.; Snyder, W.E.; Yoo,Y.; Drew, M.S. Color image processing pipeline. IEEE Signal Process. Mag. 2005, 22, 34-43. [CrossRef]

Rasmussen, C.E.; Williams, C.K.I. Gaussian Processes for Machine Learning; The MIT Press: Cambridge, MA, USA, 2006.

rawpy 0.13.1 documentation. Available online: https://letmaik.github.io/rawpy/api/ (accessed on 4 November 2019).

RawTherapee. Available online: http://www.rawtherapee.com/ (accessed on 5 February 2020).

Riutort-Mayol, G.; Marqués-Mateu, Á.; Seguí, A.E.; Lerma, J.L. Grey level and noise evaluation of a Foveon X3 image sensor: A statistical and experimental approach. Sensors 2012, 12, 10339-10368. [CrossRef]

Robert, E.; Petrognani, S.; Lesvignes, E. Applications of digital photography in the study of Paleolithic cave art. J. Archaeol. Sci. Rep. 2016, 10, 847-858. [CrossRef]

Rocha,M.; Ferreira, P.G. An Introduction to the Python Language. In Bioinformatics Algorithms; Academic Press, 2018; pp. 5-58. [CrossRef]

Rogerio-Candelera, M.A. Digital image analysis based study, recording, and protection of painted rock art. Some Iberian experiences. Digital Applications in Archaeology and Cultural Heritage 2015, 2, 68-78.

Rousseeuw, P.J. Silhouettes: a graphical aid to the interpretation and validation of cluster analysis. $J$. Comput. Appl. Math. 1987, 20, 53-65.

Ruiz, J.F.; Pereira, J. The colors of rock art. Analysis of color recording and communication systems in rock art research. J. Archaeol. Sci. 2014, 50, 338-349. [CrossRef]

Ruppert, D.; Wand, M.; Carroll, R.; Raymond J. Semiparametric regression during 2003-2007. Electron. J. Stat. 2009, 3, 1193-1256. [CrossRef]

Sarriá Boscovich E. The rock printigns of Cova Remigia (Ares del Maeste, Castellón). Lucentum An la Univ. Alicant Prehist. Arqueo.l e Hist. Antig. 1988, 7, 7-34.

Schanda, J.; Eppeldauer, G.; Sauter, G. Tristimulus Color Measurement of Self-Luminous Sources. In Colorimetry: understanding the CIE system; John Wiley \& Sons, Inc.: Hoboken, New Jersey, USA, 2007; pp. 135-158.

SciPy. Available online: https://www.scipy.org/ (accessed on 3 February 2020).

Scikit-learn. Machine Learning in Python. Available online: https://scikit-learn.org/stable/index. html (accessed on 4 November 2019).

Scikit-learn. KMeans. Available online: https://scikit-learn.org/stable/modules/generated/ sklearn.cluster.KMeans.html (accessed on 4 November 2019). 
Sesana, E.; Gagnon, A.S.; Bertolin, C.; Hughes, J. Adapting cultural heritage to climate change risks: Perspectives of cultural heritage experts in Europe. Swiss J. Geosci. 2018, 8, 305. [CrossRef]

Sharma, G. Color fundamentals for digital imaging. In Digital Color Imaging Handbook; CRC Press: Boca Raton, Florida, USA, 2003; 94.

Sharma, G.; Wu, W.; Dalal, E.N. The CIEDE2000 color-difference formula: Implementation notes, supplementary test data, and mathematical observations. Color Res. Appl. 2005, 30, 21-30. [CrossRef]

Shen, H.; Cai, P.; Shao, S.; Xin, J.H. Reflectance reconstruction for multispectral imaging by adaptive Wiener estimation. Opt. Express 2007, 15, 15545-15554. [CrossRef]

Shen, H.L.; Zhang, H.G.; Xin, J.H.; Shao, S.J. Optimal selection of representative colors for spectral reflectance reconstruction in a multispectral imaging system. Appl. Optics 2008, 47, 2494-2502. [CrossRef]

Shimano,N.; Terai,K.; Hironaga, M. Recovery of spectral reflectances of objects being imaged by multispectral cameras. J. Opt. Soc. Am. A 2007, 24, 3211-3219. [CrossRef]

Sigma Corporation. Direct Image Sensor Sigma SD15. Available online: http://www . sigma-sd.com/SD15/ technology-colorsensor.html (accessed on 10 June 2019).

Scikit-learn. Selecting the number of clusters with silhouette analysis on KMeans clustering. Available online: https://scikit-learn.org/stable/auto_examples/cluster/plot_kmeans_silhouette_ analysis.html\#sphx-glr-auto-examples-cluster-plot-kmeans-silhouette-analysis-py (accessed on 4 November 2019).

SOURCEFORGE. dcpTool and DNG Camera Profiles. Available online: http://dcptool sourceforge. net/Introduction.html (accessed on 5 February 2020)

Stevens, M.; Párraga, CA.; Cuthill, IC.; Partridge, JC.; Troscianko, TS. Using digital photography to study animal coloration.Biol. J Linn. Soc. 2007, 90, 211-237. [CrossRef]

Stone, M. Cross-validatory choice and assessment of statistical predictions. J. R. Stat. Soc. Ser. B Methodol. 1974, 2, 111-147. [CrossRef]

Sur, F.; Grédiac, M. Measuring the noise of digital imaging sensors by stacking raw images affected by vibrations and illumination flickering. SIAM J. Imaging Sci. 2015, 8, 611-643. [CrossRef]

Tang, P.; Chen, F.; Zhu, X.; Zhou, W. Monitoring cultural heritage sites with advanced multi-temporal InSAR technique: The case study of the Summer Palace. Remote Sens. 2016, 8, 432. [CrossRef]

Thomson, M.; Westland, S. Colour-imager characterization by parametric fitting of sensor responses. Color Res. Appl. 2001, 26, 442-449. [CrossRef]

Thorndike, R.L. Who belongs in the family? Psychometrika 1953, 18, 267-276.

Topcon. Download application and manual. Available online: https://www.topcon-techno.co.jp/en/ download-cat/manual/ (accessed on 4 February 2020).

UNESCO. Rock Art of the Mediterranean Basin on the Iberian Peninsula. Available online: http://whc . unesco.org/en/list/874 (accessed on $10 \mathrm{Feb} 2020$ ). 
Valero, E.M.; Nieves, J.L.; Nascimento, S.M.; Amano, K.; Foster, D.H. Recovering spectral data from natural scenes with an RGB digital camera and colored filters. Color Res. Appl. 2007, 32, 352-360. [CrossRef]

Valltorta. Cultural Park. Caves and Shelters. Available online: https://www.valltorta.es (accessed on 30 January 2020).

Van Dormolen, H. Metamorfoze Preservation Imaging Guidelines-Image Quality; Version 1.0; Society for Imaging Science and Technology: Springfield, VA, USA, 2012.

Van Poucke, S.; Vander Haeghen, Y.; Vissers, K.; Meert, T.; Jorens, P. Automatic colorimetric calibration of human wounds. BMC Med. Imaging 2010, 10, \%. [CrossRef]

Van Rossum, G.; Drake, F.L. The python language reference manual, Network Theory Ltd., 2011.

Vazquez-Corral, J.; Connah, D.; Bertalmío, M. Camera sensor response, Color characterization, Perceptual correction. Sensors 2014, 14, 23205-23229. [CrossRef]

Verivide. Digieye for textile and apparel. Available online: https: //www. verivide. com/product-list/ digieye-for-textile-and-apparel (accessed on 4 November 2019).

Vrhel, M.J.; Trussell, H.J. Color correction using principal components. Color Res. Appl. 1992, 17, 328-338. [CrossRef]

Wee, AG.; Lindsey, DT.; Kuo, S.; Johnston, WM. Color accuracy of commercial digital cameras for use in dentistry. Dental Materials 2006, 22, 553-559. [CrossRef]

Westland, S.; Ripamonti, C.; Cheung, V. Characterisation of Cameras. In Computational Colour Science Using MATLAB ${ }^{\circledR}$, 2nd ed.; John Wiley 6 Sons, Inc.: Chichester, UK, 2012; 143-15\%.

Westland, S. Computational Colour Science using MATLAB 2ed. Available online: $\quad$ https://www. mathworks. com/matlabcentral/fileexchange/ 40640-computational-colour-science-using-matlab-2e (accessed on 5 February 2020)

Westra, E. Python geospatial development.; Packt Publishing Ltd, 2013.

Wright, W.D. Professor Wright's Paper from the Golden Jubilee Book: The historical and experimental background to the 1931 CIE system of colorimetry. In Colorimetry: understanding the CIE system; John Wiley $\&$ Sons, Inc.: Hoboken, New Jersey, USA, 2007; 9-23.

Xiong, W.; Funt, B.; Shi, L.; Kim, S.S.; Kang, B.H.; Lee, S.D.; Kim, C.Y. Automatic white balancing via gray surface identification. Color Imaging Conf. 2007, 1, 143-146. [url]

X-Rite. Software. Available online: https: //xritephoto. com/software (accessed on 5 February 2020)

X-Rite. Color Measurement Software for Formulation 85 Quality Control. Available online: https: //www. xrite. com/categories/formulation-and-quality-assurance-software (accessed on 4 February 2020).

X-Rite. CM-SA Skin Analysis Software. Available online: https://sensing. konicaminolta.us/ products/cm-sa-skin-analysis-software/ (accessed on 4 February 2020).

X-Rite. ColibriR. Available online: https://sensing. konicaminolta. us/products/ colibri-color-softwarel (accessed on 4 February 2020). 
X-Rite. Color-EyeR. Available online: https://www.xrite.com/categories/ color-matching-apps/coloreye (accessed on 4 February 2020).

Xu, P.; Xu, H.; Diao, C.; Ye, Z. Self-training-based spectral image reconstruction for art paintings with multispectral imaging. Appl. Optics 2017, 56, 8461-8470. [CrossRef]

Yamakabe, R.; Monno, Y.; Tanaka, M.; Okutomi, M. Tunable color correction between linear and polynomial models for noisy images. In Proceedings of the IEEE International Conference on Image Processing (ICIP), Beijing, China, 2017; 3125-3129. [CrossRef]

Yastikli, N. Documentation of cultural heritage using digital photogrammetry and laser scanning. Journal of Cultural Heritage 2007, 8, 423-42\%. [CrossRef]

Yoon, C.R.; Cho, M.S. Colorimetric characterization for digital camera by using multiple regression. In Proceedings of the IEEE Region 10 Conference, TENCON 99, 'Multimedia Technology for Asia-Pacific Information Infrastructure' (Cat. No.99CH37030), Cheju Island, Korea, 1999; 1, 585-588. [CrossRef]

Zambelli, P.; Gebbert, S.; Ciolli, M. Pygrass: An Object Oriented Python Application Programming Interface (API) for Geographic Resources Analysis Support System (GRASS) Geographic Information System (GIS). ISPRS Int. J. Geo-Inf. 2013, 2, 201-219. [CrossRef]

Zhang, X.; Wang, Q.; Li, J.; Zhou, X.; Yang, Y.; Xu, H. Estimating spectral reflectance from camera responses based on CIE XYZ tristimulus values under multi-illuminants. Color Res. Appl. 2017, 42, 68-7\%. [CrossRef]

Zhang, Y.; Wang, G.; Xu, J. Parameter Estimation of Signal-Dependent Random Noise in CMOS/CCD Image Sensor Based on Numerical Characteristic of Mixed Poisson Noise Samples. Sensors 2018, 18, 2276-2293. [CrossRef]

Zhao, Y.; Berns, R.S. Image-based spectral reflectance reconstruction using the matrix $R$ method. Color Res. Appl. 2007, 32, 343-351. [CrossRef] 
